\title{
Liquid Crystal Microswimmers \\ - from single entities to collective dynamics -
}

\author{
DISSERTATION \\ zur Erlangung des mathematisch-naturwissenschaftlichen Doktorgrades \\ "Doctor rerum naturalium" \\ der Georg-August-Universität Göttingen \\ im Promotionsprogramm ProPhys \\ der Georg-August University School of Science (GAUSS)
}

\section{vorgelegt von \\ Carsten Krüger}

aus Bielefeld

Göttingen, 2016 


\section{Betreuungsausschuss:}

Prof. Dr. Stephan Herminghaus, Dynamik komplexer Fluide, Max-Planck-Institut für Dynamik und Selbstorganisation, Göttingen

Prof. Dr. Jörg Enderlein, Drittes Physikalisches Institut, Universität Göttingen

Dr. Christian Bahr, Dynamik komplexer Fluide, Max-Planck-Institut für Dynamik und Selbstorganisation, Göttingen

\section{Mitglieder der Prüfungskommision:}

Referent: Prof. Dr. Stephan Herminghaus, Dynamik komplexer Fluide, Max-Planck-Institut für Dynamik und Selbstorganisation, Göttingen

Koreferent: Prof. Dr. Jörg Enderlein, Drittes Physikalisches Institut, Universität Göttingen

\section{Weitere Mitglieder des Prüfungsausschuss:}

Dr. Christian Bahr, Dynamik komplexer Fluide, Max-Planck-Institut für Dynamik und Selbstorganisation, Göttingen

Prof. Dr. Christoph Schmidt, Drittes Physikalisches Institut, Universität Göttingen

Prof. Dr. Sarah Köster, Institut für Röntgenphysik, Universität Göttingen

Prof. Dr. Annette Zippelius, Institut für Theoretische Physik, Universität Göttingen

Tag der mündlichen Prüfung: 02. November 2016 


\section{Contents}

List of Tables

List of Figures

1 Introduction 1

2 Theory 5

2.1 Swimming at low Reynolds numbers . . . . . . . . . . . . . . . 5

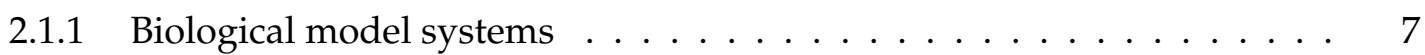

2.1.2 Artificial swimmer systems . . . . . . . . . . . . . . . . 10

2.2 Liquid crystal phases . . . . . . . . . . . . . . . . . . . 12

2.3 Propulsion of Marangoni-powered emulsions . . . . . . . . . . . . . . 15

2.4 Flow fields generated by droplet swimmers . . . . . . . . . . . . . . 19

2.5 Brownian motion \& mean squared displacement . . . . . . . . . . . . 22

3 Materials 27

3.1 Liquid crystals . . . . . . . . . . . . . . . . . . . . 27

3.2 Ionic surfactants . . . . . . . . . . . . . . . . . . . 28

4 Methods 31

4.1 Microfabrication . . . . . . . . . . . . . . . . . . . . 31

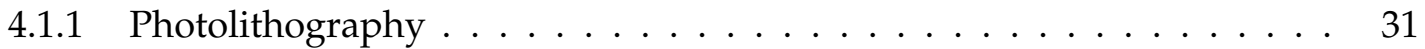

4.1 .2 Experimental reservoirs . . . . . . . . . . . . 32

4.2 Droplet production in microfluidics . . . . . . . . . . . . . . . 33

4.3 Polarized Optical Microscopy . . . . . . . . . . . . . . . . . . 36

4.4 Light-Sheet Microscopy $\ldots \ldots \ldots \ldots \ldots \ldots \ldots$

4.5 Particle tracking . . . . . . . . . . . . . . . . . . 44

4.5 .1 Image processing . . . . . . . . . . . . . . . 44

4.5 .2 Trajectory reconstruction $\ldots \ldots \ldots \ldots \ldots$

4.6 Determination of flow profiles . . . . . . . . . . . . . . 45

5 Results 49

5.1 Droplet motion in one dimension . . . . . . . . . . . . . . . . 49

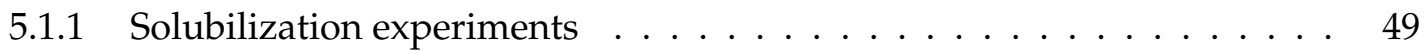

5.1 .2 1D movement in capillaries . . . . . . . . . . . . . . 53

5.1 .3 Estimation of Marangoni strength $\ldots \ldots \ldots \ldots \ldots$

5.1 .4 Effect of chemotaxis . . . . . . . . . . . . . . 57 
5.2 Dilute systems in two dimensions . . . . . . . . . . . . . 63

5.2.1 Hydrodynamic flow profile . . . . . . . . . . . . . . . 63

5.2 .2 Diameter dependent velocity . . . . . . . . . . . . . . . . . . . . . . . 66

5.2 .3 Movement threshold . . . . . . . . . . . . . . . . . . . 69

5.2 .4 Wall interactions ................... 70

5.2 .5 Curling liquid crystal swimmers . . . . . . . . . . . . 76

5.3 Dilute systems in three dimensions . . . . . . . . . . . . . . . . . . 84

5.4 Dense systems in two and three dimensions . . . . . . . . . . . . . . 87

5.4.1 Influence of the dimensionality on the collective behaviour . . . . . . . 88

5.4.1.1 Qualitative observations in dense systems . . . . . . . . . 89

5.4.1.2 Morphology of individual clusters . . . . . . . . . . 90 90

5.4.1.3 Effect of confinement on the clustering behaviour . . . . . . . 94

5.4.2 Influence of buoyancy on collective behaviour . . . . . . . . . . 96

5.4.2.1 Effect of varying the buoyancy on the clustering behaviour . . 96

5.4.2.2 High-density suspensions in LSM . . . . . . . . . . . . . 98

6 Conclusions

103

7 Ongoing research $\quad 109$

References $\quad 113$ 


\section{List of Tables}

2.1 Diffusion constants and persistence times. . . . . . . . . . . 25

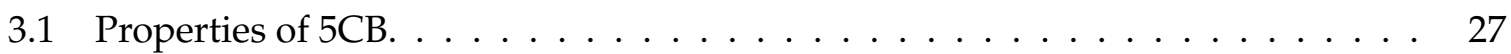

3.2 Properties of ionic surfactants. . . . . . . . . . . . . . . . 29

5.1 Parameters for the Todorov model. . . . . . . . . . . . . . . . . 52

\section{List of Figures}

1.1 An overview on collective behaviour in active systems. . . . . . . . . 2

2.1 Schematic illustration of the scallop theorem. . . . . . . . . . . . . . 6

2.2 Collection of biological swimmers to scale. . . . . . . . . . . . . 7

2.3 Chemotactic guided trajectories of E.coli and V.alginolyticus. . . . . . . . . . . . 8

2.4 Two exemplary microrobots utilizing the two modes of propulsion proposed by Purcell. . . . . . . . . . . . . . . . . . . . . 10

2.5 Two mechanisms generating Marangoni flow. . . . . . . . . . . . . . . . . . . 11

2.6 Anisotropy of molecular shape and phases depending on temperature. . . . . . 12

2.7 Order parameter $S$ vs. temperature for 5 CB $\ldots \ldots \ldots \ldots \ldots$

2.8 Anisotropy of viscosity in nematic liquid crystals. . . . . . . . . . . . . . 14

2.9 Emerging defect structures due to different anchoring on LC droplet interfaces. 15

2.10 Two pathways for solubilization of oil in surfactant solutions. . . . . . . . . . . 16

2.11 Concentration profiles for both mechanisms and the evolving depletion with

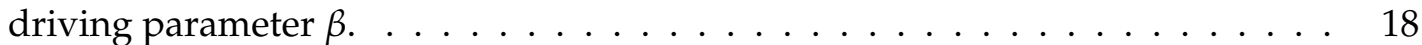

2.12 Schematic of flow field around and inside a moving droplet. . . . . . . . . . . 21

2.13 Schematic of squirmer types and their respective surface flow velocity profiles. 22

2.14 MSD of Janus particles. . . . . . . . . . . . . . . . . . . 25

3.1 Collected values for TTAB micelles. . . . . . . . . . . . . . . . . 30

4.1 Schematic of photolithography . . . . . . . . . . . . . 31

4.2 Process of droplet generation. . . . . . . . . . . . . . . . . 33

4.3 Schematic representation of microfluidic flow-focusing device. . . . . . . . . 34

4.4 Properties of droplets produced in microfluidics. . . . . . . . . . . . . . 35

4.5 Schematic of POM. . . . . . . . . . . . . . . . . 37

4.6 Example of POM. . . . . . . . . . . . . . . . . . . . . . 38

4.7 Schematic illustration of a focused Gaussian beam. . . . . . . . . . . . . . . . 39

4.8 Characteristics of NileRed. . . . . . . . . . . . . . . . . . . . . . . . 40

4.9 Schematic illustration of $\mathrm{LSM} \ldots \ldots \ldots \ldots \ldots \ldots$

4.10 Density calibration of surfactant solutions. . . . . . . . . . . . . . . 42 
4.11 Measurement of light-sheet dimensions. . . . . . . . . . . . . . . . . . 43

4.12 Example for PSV analysis. . . . . . . . . . . . . . . . . . 47

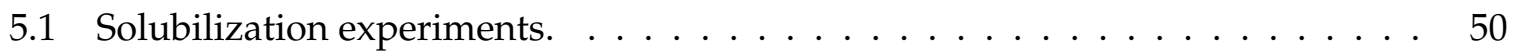

5.2 Velocity and MSD from 1D experiments. . . . . . . . . . . . . . . 553

5.3 Collision event in capillary with director orientations and the local velocity. . . 54

5.4 Temperature dependent propulsion in 1D . . . . . . . . . . . . . 55

5.5 Estimation of interfacial tension gradients. . . . . . . . . . . . 57

5.6 Experiment highlighting the chemotactic behaviour of swimming LC droplets. 58

5.7 Maze-solving experiment of chemotactic LC swimmers. . . . . . . . . . . 58

5.8 Trajectory length of maze solving droplets. . . . . . . . . . . . . . . . 59

5.9 Trajectories with autochemotactic repulsion. . . . . . . . . . . . . . . 60

5.10 Optical investigation of the deposited trail. . . . . . . . . . . . . . . . . 61

5.11 Autochemotactic experiment along pillar structures. . . . . . . . . . . . . 62

5.12 Autochemotactic experiment in a branching channel. . . . . . . . . . . 63

5.13 Hydrodynamic flow profile. . . . . . . . . . . . . . . . . . 64

5.14 Tangential velocity $v_{\theta}$ vs. angle $\theta$ and vs. the distance $r / R$ from the droplet's surface............................ 65

5.15 Loopy tracer scattering by a force-free swimmer. . . . . . . . . . . . . . 66

5.16 2D schematic illustration and velocity for various surfactant concentrations. . . 67

5.17 Velocity vs. diameter and the cruising range of the system. . . . . . . . . . . 68

5.18 Movement threshold for $125 \mu \mathrm{m}$ sized droplets in 2D . . . . . . . . . . . . . . 69

5.19 Experimental determination of wall attraction and activity of the system. . . . 71

5.20 Attachment length $l$ and wall detention time $\tau_{d}$ for droplets in circular confine-

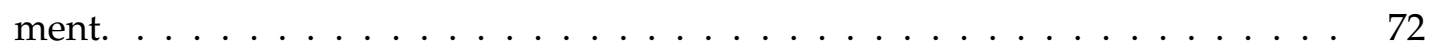

5.21 Wall detention time and attachment length for rectangular reservoirs. . . . . . 73

5.22 Influence of confinement on droplet behaviour and flow field. . . . . . . . . . 74

5.23 Velocity profile in different confinements. . . . . . . . . . . . . . . . 75

5.24 MSD and angular autocorrelation $C(\tau) \ldots \ldots \ldots \ldots \ldots$

5.25 Example of curling trajectories. . . . . . . . . . . . . . . 78

5.26 Averaged phase shift and PTV results close to boundary. . . . . . . . . . . 79

5.27 Three dimensional trajectories in the light-sheet microscope. . . . . . . . . . . 80

5.28 Example of 3D helical trajectories. . . . . . . . . . . . . . . . . 81

5.29 Meandering trajectories in $2 \mathrm{D}$ confinement. . . . . . . . . . . . . 82

5.30 Comparison of curling and meandering trajectories. . . . . . . . . . . . . 83

5.31 Dilute systems in Light Sheet Microscopy. . . . . . . . . . . . . . . . . . . 85

5.32 MSD and correlation function from helical trajectories. . . . . . . . . . . 86

5.33 Trajectories in LSM with applied magnetic field. . . . . . . . . . . . . . . 87

5.34 Schematic illustration of the experimental chamber. . . . . . . . . . . . . 88 
5.35 Collective droplet behaviour determined by dimensional confinement. . . . . . 89

5.36 Time lapse of one clustering experiment over the course of $30 \mathrm{~min}$. . . . . . . 90

5.37 Polarized image of stable cluster and clustering stabilized by a convection roll, schematic and experimental proof. . . . . . . . . . . . . . 91

5.38 Cluster radius $R$ vs. hovering height $h \ldots \ldots \ldots \ldots \ldots$. . . . . . . 93

5.39 Minimal droplet number for clustering. . . . . . . . . . . . . . . 93

5.40 Average cluster distance $\Lambda$ for different reservoir heights $H$. . . . . . . . 95

5.41 Hovering height $h$ as a function of swimmer velocity $v_{\text {free }} \ldots \ldots \ldots$

5.42 Effect of buoyancy adjustments on clustering behaviour. . . . . . . . . . 97

5.43 Pattern formation influenced by dimensionality in a two-layer reservoir geom-

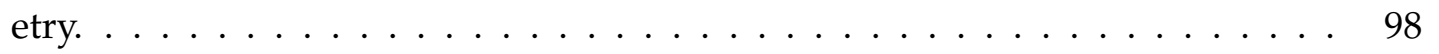

5.44 High density suspensions in LSM. . . . . . . . . . . . . . . . 99 



\section{Abstract}

Recently, artificial self-propelled systems at low Reynolds numbers have gained a lot of attention. The main interest lies in mimicking well-studied systems, e.g. the active Brownian motion of bacteria, or in gaining deeper insight into nature's schemes like search of food or light. Moreover, fundamental and hitherto unanswered questions like the Plankton paradox prevail. Due to the complexity of living (eco-)systems, computational simulations or experiments in nature can often only tackle parts of the underlying mechanisms. In all these aspects, reproducible artificial microswimmers in well controlled laboratory conditions offer a possibility to gain insights into these open questions. Hitherto, preceding studies on artificial systems mainly focused on Janus particles. These introduce an intrinsic symmetry breaking onto the system, and often propel due to external addition of energy.

The presented thesis discusses a well controllable and adaptable artificial system, consisting of nematic liquid crystal (LC) droplets in ionic surfactant solutions above the critical micelle concentration. In a spontaneously induced process, the droplets undergo micellar solubilization. If the surfactant concentration surpasses a threshold, this is accompanied by self-sustained propulsion, with the incorporation of the LC molecules in the surfactant micelles as the energy source. The active emulsion system generates a symmetry breaking on its own due to an inhomogeneity in surfactant coverage along the droplet interface. This generates Marangoni flows in an otherwise fully symmetric system.

The main advantages of our system are the well-studied microfluidic droplet preparation, active swimming periods up to hours and the tuneability of the buoyancy. The droplet system is therefore a prime candidate for studying the behaviour in various nature-like environments.

This thesis provides systematic studies covering a substantial range of the parameter space of interest in terms of dimensionality, driving force and number density of entities. Through the investigation of the solubilization behaviour, the propulsion of the droplets is linked to the molecular pathway of solubilization. In this respect, the (auto)chemotactic behaviour of the droplets is discussed by investigating maze solving and avoidance dynamics. The analysis of one-dimensional propulsion in capillaries is followed by the investigation of the hydrodynamic flow profile and wall interactions in two dimensions. One main aspect is the influence of the nematic nature of the droplets on the characteristic motion. It is found that both, curling in 2D and helical swimming in 3D, result from a second symmetry breaking in the system. The latter arises from the interaction of the nematic matrix with internal flows. Yet, this behaviour vanishes for temperatures exceeding the nematic-to-isotropic transition, giving rise to ballistic motion. Eventually, dense suspensions of droplets are examined in three dimensions, showing convection-roll stabilized large scale clustering when buoyancy is present, while in freely floating suspensions with buoyancy set to zero, autochemotactic caging dynamics occur. 



\section{Zusammenfassung}

Künstliche Mikroschwimmer bei kleinen Reynoldszahlen erlangten in den letzten Jahren große Aufmerksamkeit. Der Schwerpunkt liegt hierbei auf der Nachahmung von Verhaltensmustern bekannter biologischer Systeme, z. B. der aktiven Brown'schen Bewegung von Escherichia coli Bakterien, oder in der Erlangung eines tieferen Verständnisses der in der Natur vorkommenden Strategien für die Suche nach Nahrung oder Licht. Des Weiteren sind fundamentale Fragestellungen in biologischen Systemen, z. B. das Plankton paradoxon, noch unbeantwortet. Durch die intrinsische Komplexität der Systeme können diese Fragen nicht mit Computersimulationen oder Experimenten in der Natur behandelt werden, wohingegen künstliche Schwimmer unter reproduzierbaren Laborbedingungen einen möglichen Lösungsansatz bieten. Bisher bestehen künstliche Systeme zumeist aus Janus-Teilchen. Diese besitzen eine intrinsische Asymmetrie und müssen häufig durch eine externe Energiequelle angetrieben werden.

Die vorliegende Dissertation beschreibt ein kontrollierbares und gleichzeitig variables künstliches System, bestehend aus aktiven Emulsionen. Die Grundbausteine sind aus einem nematischen Flüssigkristall bestehende Öl-Tropfen, die in eine ionischen Tensidlösung mit Konzentrationen oberhalb der kritischen Mizellbildungskonzentration gegeben werden. Die Tropfen lösen sich in einem spontanen, mizellären Prozess auf. Überschreitet die Tensidkonzentration einen Schwellwert, wird neben der Löslichkeit auch ein Eigenantrieb induziert. Hierbei liefert der Lösungsprozess die Energie für die Fortbewegung. Dieses System aktiver Emulsionen erfährt eine selbstgenerierte Symmetriebrechung durch eine inhomogene Belegung der Tropfenoberfläche mit Tensidmolekülen. Daraus entsteht ein Marangoni-Fluss in einem ansonsten vollständig symmetrischen System.

Vorteile dieses Systems sind gegeben durch zuverlässige, mikrofluidische Strategien zur Herstellung von Emulsionen, aktive Perioden der Tropfen bis hin zu Stunden und die Möglichkeit, die Auftriebscharakteristika anzupassen. Diese Eigenschaften zeichnen das System als geeigneten Kandidaten für die Untersuchung von biologischen Systemen aus.

Die Experimente in dieser Arbeit decken einen großen Bereich des Parameterraums hinsichtlich Dimensionalität, Antriebskraft und Anzahl der Schwimmer ab und liefert dabei systematische Studien hinsichtlich differenzierter Systemparameter.

Die Untersuchung des Löslichkeitsverhaltens resultiert in der Erkenntnis, dass der Lösungsprozess dem molekularen Schema folgt. In dieser Hinsicht werden (auto)chemotaktische Verhaltensmuster in Labyrinthstrukturen und anhand des Meidungsverhaltens der Tropfen untersucht. Der Analyse der eindimensionalen Fortbewegung in Glaskapillaren folgt die hydrodynamische Untersuchung des Flussfelds und der Wandinteraktionen in zwei Dimensionen. 
Einer der Hauptaspekte der Arbeit behandelt den Einfluss des nematischen Direktorfeldes auf die Bewegungscharakteristik der Tropfen. Hierbei wird eine zweite Symmetriebrechung durch die Wechselwirkung zwischen dem Direktorfeld und dem internen Flussfeld erzeugt. Daraus resultieren charakteristische kreisende Bewegungen in 2D und helikale Trajektorien in 3D. Oberhalb des nematisch/isotropen Phasenübergangs werden diese Muster unterdrückt und die Tropfen bewegen sich auf ballistischen Pfaden mit großer Persistenzlänge. Abschliessend werden dichte Schwimmersuspensionen betrachtet. Es zeigt sich, dass die Clusterbildung von sedimentierenden Tropfen durch Erzeugung einer Konvektionsrolle stabilisiert wird. Wird der Auftrieb hingegen angepasst, sodass die Tropfen schweben, entstehen Käfigdynamiken durch autochemotaktische Verhaltensmuster und Stoßinteraktionen. 


\section{Introduction}

The present thesis is driven by the fundamental curiosity of scientists to understand the mechanisms of life, where locomotion and interaction of organisms play a fundamental role. Even though scientists have understood how single birds can fly or single fish can swim, the collective mechanisms that lead to formation of flocks and schools of many entities still eludes us. When going down in scale to microscopic objects, even the simple propulsion schemes of macroscopic systems, like opening and closing the shells of a scallop, cannot be adapted. Swimming at the microscale in liquids implies a transition of regimes in terms of fluid friction, viscosity and inertia, described by the unit free Reynolds number. While the motion of birds and fish is dominated by inertia, the motion of bacteria, algae or sperm is subject to friction and viscosity. This results in various sophisticated propulsion schemes in nature. Many of these schemes involve beating or rotating of flagella or cilia to overcome the viscous drag [1].

Under this aspect, the bacterium Escherichia coli (E.coli) is probably the best studied organism undergoing active Brownian motion. The latter term implies the superposition of active propulsion and Brownian diffusion due to the small sizes of the entities. The influence of diffusion onto the emerging motion characteristics depends not only on the translatory diffusion constant but furthermore on the rotational diffusion constant of the swimmer. Moreover, most organisms have developed strategies to find sources of food, light or oxygen, referred to as chemotaxis. For example, E.coli bacteria move in a run-and-tumble fashion along chemical gradients with high efficiency [2], whereas cells of Chlamydomonas reinhardtii rotate slowly around their long axis to detect light intensity changes by their eyespot [3].

Hitherto, even the swimming of single bacteria poses open questions, although they seldom swim individually in nature. In assemblies, the swimmers reach new levels of complexity, either through formation of colonies, networks, biofilms or due to the huge variety of plankton species in the ocean currents. The underlying mechanisms range from purely physical interactions, e.g. hard-core repulsion or hydrodynamic interactions, to fully biological mechanisms, like chemotaxis or olfactory and visual signals [4, 5]. These large-scale phenomena are far from being understood and call for practicable experimental investigations.

Fig. 1.1 allows to catch a glimpse of the span of sizes of swimmers and the number of interacting species in collective dynamics. In the depicted examples, the sizes of the single entities range from tens of centimetres down to micrometres. Yet, up to thousands of entities move cooperatively in large ensembles, resulting in intriguing phenomena.

In the last decade, the effort of producing artificial swimmer systems to investigate and understand the underlying mechanisms in nature has increased drastically. Most strongly in demand are systems with well controllable and adjustable interactions which can be fully de- 

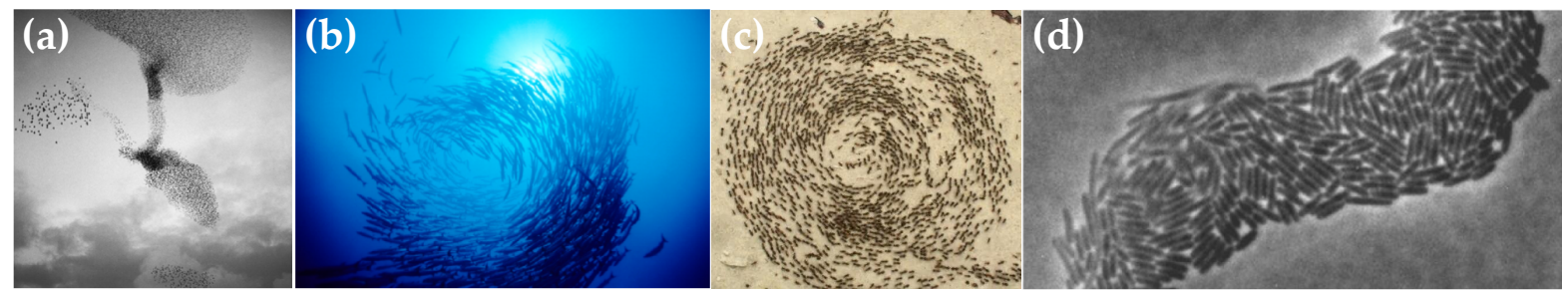

Fig. 1.1: Collective behaviour on length scales ranging from tens of meters down to tens of microns (from left to right). (a) Flock of birds, (b) school of fish, (c) ant mill and (d) bacterial colony. Images (a), (b) and (d) adapted from [6]. Image (c) taken from [7].

scribed by physical models. This restriction is inevitable to distinguish single properties in living cells, i.e. between a purely physical phenomenon and the biological impact.

Computer simulations can only provide simplified models, constraining either the shape or interactions of the swimmers. Yet, large-scale systems are nearly impossible to compute, due to their spatial extent, large number of involved entities and long range hydrodynamic interactions. The latter only decay algebraically, making it difficult to choose a reliable cut-off for the mutual interactions [4].

Therefore, experimental systems are of vital importance, not just as benchmarks to validate available computer simulations but also to increase the extent of study. In the endeavour for artificial swimmer systems, the availability of energy and the subsequent conversion into a propulsive force pose the main difficulties which have to be overcome. The first approaches to tackle the intriguing questions of nature were realized in granular systems and by micromotors with external agitation, e.g. induced by vibrating the samples [8, 9] or by applying dynamic magnetic fields [10]. Many of the micromotors mimic biological organisms, for example a helical screw resembling the beating pattern of E.coli [11] or Purcell's famous scallop [12].

One of the main approaches involves self-induced phoretic forces to achieve displacement of colloids. Depending on the created gradients, different types of motion are distinguished, for example diffusio-, electro- or thermophoretic motion [13]. Many studies concerning the diffusio- or thermophoresis of Janus particles have been reported on [14-16]. Although this is a remarkable system for observing active Brownian motion in various conditions, the drawbacks of sedimentation of the colloids and the necessity of an external energy supply prevail and hence, the system misses to bridge the gap between artificial and living systems.

Thus, studies on active emulsions, droplets immersed in a second immiscible fluid, have emerged recently [4, 17,-19]. Active droplets, which are radially symmetric at rest, spontaneously generate interfacial tension gradients that lead to propulsion. Since no external energy input is necessary, resulting in a truly autonomous motion with hydrodynamic flow 
fields that are comparable to bacteria or plankton species, active emulsions are of special interest. As a suitable driving mechanism, the Marangoni effect is utilized. This mechanism generates surface flows to equilibrate interfacial tension inhomogeneities [20]. These deviations are obtained by either chemically changing the activity of surfactant molecules appropriately or by solubilization processes, resulting in an asymmetric depletion of surfactants at the surface of the droplet [4]. The first mechanism provides a wide range of tuneability concerning the chemical reactions. However, the reaction products often interfere with unbiased propulsion or the "fuel" incorporated inside the droplet limits the propulsion duration. Concerning these aspects, the second mechanism of solubilizing active emulsions promises better performance. Nevertheless, the number of reported systems relying on this mechanism is limited so far [19]. The key aspect in this case is a finite solubility of the droplet material in the surrounding bulk liquid, such that the droplet medium in itself is the "fuel" and the diameter of the droplet will shrink in size over time.

Through the outlined adaptability of active emulsions, numerous life-like aspects are imaginable. For example, every swimming entity can provide a reservoir for carrying cargo and even respond to chemical gradients for guiding the droplets to specific places. Both aspects are key features for possible applications, e.g. drug delivery in medicine and pharmacy.

The system of active emulsions is a prime candidate for mimicking biological systems, since a large number of identical droplets can be generated in microfluidic devices. Moreover, the swimming periods can reach hours and, besides the mentioned chemotactic behaviour, external stimuli e.g. temperature or magnetic fields can control the droplet behaviour.

In nature, the interplay of buoyancy, advection and propulsion is of utter importance. Hence, the presented system in this thesis is tuned in buoyancy by adjusting the density of the continuous phase. In accordance to the selected buoyancy, droplets sediment, float or swim freely in three dimensions.

The thesis is outlined as follows: In Chapter 2, the theoretical basics of this project are elucidated. Starting from swimming at low Reynolds numbers, the biological systems to be mimicked will be presented in more detail and previous studies on artificial systems will be highlighted. Brief summaries of liquid crystal theory and of surface-active agents are appended. The chapter concludes with a detailed description of the Marangoni-powered model system at hand. Here, the underlying propulsion mechanism, the hydrodynamic flow field expected for a spherical swimmer, the basic mathematical description for Brownian motion and analysis tools for active propulsion are outlined.

Chapter 3 covers the used materials and their properties, namely the liquid crystal 5CB and the ionic surfactants, with a focus on the cationic surfactant TTAB. In both cases, important physical parameters are listed. 
Chapter 4 introduces the applied methods. This section is of special importance, since a wide range of different applications is necessary for covering all aspects of the system. The starting points are the device fabrication with photolithography and the production of droplets in a microfluidic environment. Optical tools, polarized optical microscopy and light sheet microscopy for data recording in two and three dimensions, are introduced. Processing of the data to obtain droplet trajectories will be elucidated, followed by methods to determine the hydrodynamic flow profile in the vicinity of a single droplet.

Chapter 5 contains the main results of this study, starting from basic experiments on solubilization for a better understanding of this mechanism. Subsequently, the one-dimensional propulsion of droplets depending on surfactant concentration is investigated. Here, the chemotactic behaviour of the system is exploited for guiding droplets through mazes and autochemotactic effects are analysed.

Hereafter, the hydrodynamic flow profile in the vicinity of a droplet is determined. Thus, not only the squirmer type is specified but insights into the interaction behaviour are gained, tested with different obstacles and reservoir geometries.

In two dimensional reservoirs, a curling behaviour of the droplets emerges, reminiscent of flagellated helical swimmers. The fundamental mechanism is based on the interplay of the nematic matrix of the droplets with internal convective flows, generating a torque and resulting in the curling trajectories. Remarkably, droplets exhibit ballistic propulsion with large persistence lengths when heated above the nematic-to-isotropic transition.

In three dimensions, this behaviour results in helical trajectories with radii and period times of rotations that are comparable to two-dimensional measurements.

Dilute systems already show a plethora of remarkable behaviours but large-scale dynamics are fundamentally important in understanding biological systems. Therefore, suspensions at high number densities in unconfined reservoirs are studied. The resulting collective dynamics at length scales far beyond single droplet diameters show the formation of clusters that are stabilized by convection-rolls if buoyancy is sufficiently strong. If buoyancy is lowered, the clustering is inhibited and droplets move freely in three dimensions. Thus, the droplets are only constricted by interactions with other single entities, repulsive or autochemotactic, resulting in caging dynamics.

Chapter 6 concludes the present thesis and recovers the gained insights.

Chapter 7 offers a perspective outlook on ongoing studies and future questions to be tackled. 


\section{Theory}

In this chapter, the theoretical basis for this thesis will be set. As a first step, a general description of swimming at low Reynolds numbers will be provided. This includes a few selected examples for both, biological and artificial systems. Then, the topic is changed to the properties of our droplet material, including a brief liquid crystal theory to subsequently describe the model system at hand. The foundation in theory and mathematical analysis techniques of active Brownian motion, applied to the case of micrometer sized swimmers, will conclude this chapter.

\subsection{Swimming at low Reynolds numbers}

In our environment, many different schemes for locomotion are present. This includes not only motorized movements, e.g. of airplanes, trains and cars, but also humans propulsion, e.g. running or swimming. All these examples have in common that they are in a regime where inertia plays a major role. Stop the engine of a car and it will still move along until friction will decelerate it, stop swimming and you will still glide on for a few seconds. In a regime where inertia plays a minor role, completely different locomotion mechanisms are necessary. As an example, constant motion in a time-irreversible manner is necessary for propulsion, otherwise net forward motion is impossible. This famous story was first told by Purcell in 1977 [21].

The parameter describing the different regimes is the Reynolds number $R e$, a dimensionless parameter comparing inertial with viscous forces:

$$
\operatorname{Re}=\frac{\text { inertial forces }}{\text { viscous forces }}=\frac{\rho v^{2} / d}{\eta v / d^{2}}=\frac{d v \rho}{\eta} \text {. }
$$

Here, $d$ is a characteristic length scale, i.e. the diameter of a particle, $v$ is the velocity of the object, $\rho$ is the density of the fluid and $\eta$ is the dynamic viscosity of the surrounding fluid. The critical transition is at $R e=1$. Above this limit, the system is dominated by inertia and below, viscous forces prevail. A human breaststroke swimmer is approximately at $R e \approx 10^{4}$ while many microswimmers, biological or artificial, are at $R e \approx 10^{-4}$. A prominent example to illustrate the importance of swimming mechanisms at low Reynolds numbers is Purcell's scallop theorem [21]. The scallop moves by opening the shells slowly and closing them fast while squirting water out. This simple mechanism bases on inertia and would therefore not work at low Reynolds numbers. Furthermore, this emphasizes that breaking the time-reversal symmetry is necessary for propulsion of micron sized swimmers since the reciprocal changes 
(a)

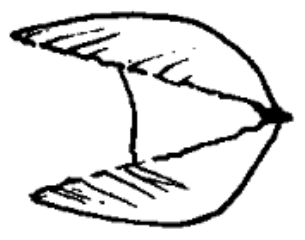

(b)

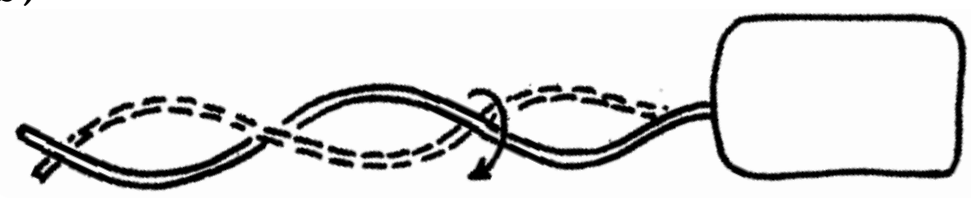

Fig. 2.1: (a) Schematic illustration of a scallop and (b) of a bacterium moving by helical rotation of a flagellum as drawn by Purcell [21]. The scallop will not move at low Reynolds numbers, while the non-reciprocal propulsion in the case of (b) will result in a net-movement of the bacterium.

in shape of the scallop will not result in net-propulsion at low Reynolds numbers.

The regime at low Reynolds numbers does not only influence the propulsion mechanisms but also the fundamental equations for the description of Newtonian fluids. The well known Navier-Stokes equation, describing the dynamics of incompressible fluids quantitatively, is given by [22]

$$
\rho \frac{\partial \mathbf{v}}{\partial t}+\rho(\mathbf{v} \cdot \nabla) \mathbf{v}=-\nabla p+\rho \mathbf{f}+\eta \nabla^{2} \mathbf{v}
$$

with $\mathbf{v}=\mathbf{v}(\mathbf{r}, t)$ as the velocity field, the pressure gradient $\nabla p$ and the body force $\mathbf{f}$ per unit volume of the fluid. For the overdamped case of $\operatorname{Re} \ll 1$, the inertia term $\rho(\mathbf{v} \cdot \nabla) \mathbf{v}$ is small as compared to the viscous term $\eta \nabla^{2} \mathbf{v}$. Thus, the dissipative terms on the left hand side of eq. (2.2) are negligible. With $\nabla \cdot \mathbf{v}=0$ for incompressible fluids, eq. (2.2) reduces to the time independent Stokes equation:

$$
0=-\nabla p+\rho \mathbf{f}+\eta \nabla^{2} \mathbf{v}
$$

The lack of dissipative terms shows that also the time inverted velocity field $-\mathbf{v}$ solves eq. (2.3) by accordingly inverted pressure and external forces. Hence, eq. (2.3) furthermore demonstrates the mentioned necessity of a non-reciprocal propulsion scheme. Thus, the aforementioned scallop would not move forward but a rotating helical movement of a flagellum would result in propulsion (see Fig. 2.1] [21].

In the following, biological model swimmers and their different propulsion schemes are introduced. Subsequently, artificial entities for mimicking various of the biological counterparts will be listed that have recently been reported on. 


\subsubsection{Biological model systems}

In nature, micrometer sized swimming organisms evolved various propulsion mechanisms, usually based on beating flagella, cilia or shape changes of filaments. Fig. 2.2 shows a collection of different swimmers. Already this representation of a small group shows the variety of body sizes, beating mechanisms and locations of the flagella. A brief introduction of the various schemes includes the reasoning of Lauga et al. [1] and is followed by a more detailed consideration of single bacteria types and their abilities. The biggest organism in this group is the

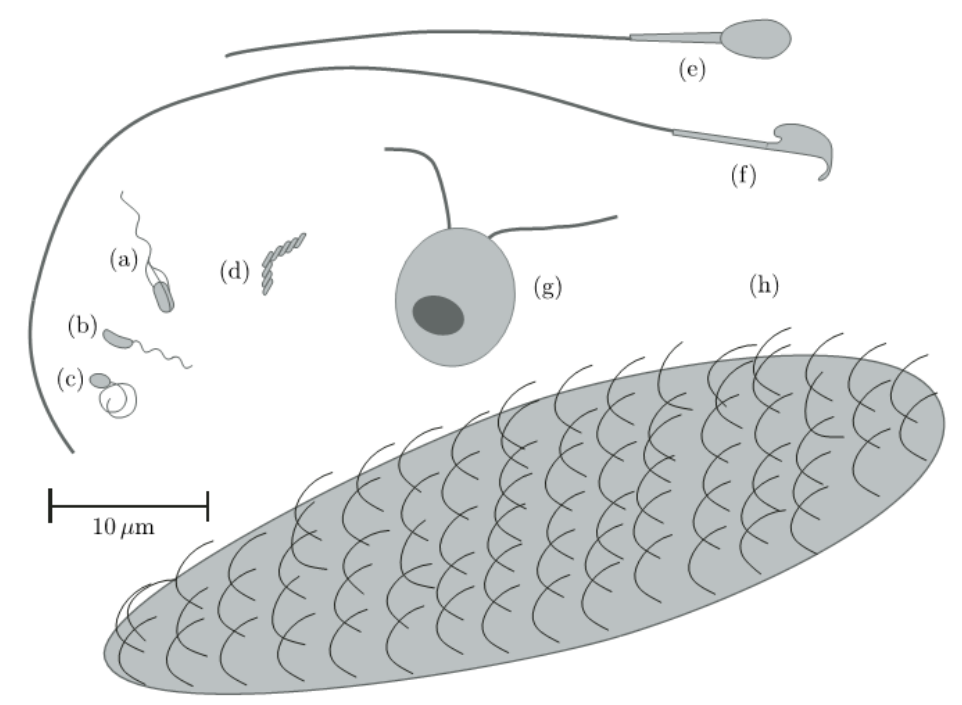

Fig. 2.2: Collection of biological swimmers to scale [1]. (a) E. coli. (b) C. crescentus. (c) R. sphaeroides, with flagella in the coiled state. (d) Spiroplasma, two regions of right-handed and left-handed coiling are separated by a single kink. (e) Human spermatozoon. (f) Mouse spermatozoon. (g) Chlamydomonas reinhardtii. (h) A small Paramecium.

pelled by flagella located at the rear end. For example, the spermatozoa of many species propel with long flagella undergoing whip-like motion. Other organisms rotate a helical flagellum for propulsion, like C. crescentus (Fig. $2.2 \mathrm{p}$ ). The right-handed helix generates forward thrust when rotated clockwise and pulls the body when rotated counterclockwise. The rotary motor of R. sphaeroides (Fig. 2.2.) turns only clockwise but stops from time to time, resulting in a compact filament coil. Probably the most common studied organism is E.coli (Fig. 2.2.). Here, a bundle of left-handed helical filaments is attached to rotary motors by a hook. When the motors turn counterclockwise, the single filaments bundle and the organism is propelled. If a single or multiple motors reverse, these filaments leave the bundle and change handedness of the helical orientation, resulting in a change of the swimming direction. Due to this mechanism, the propulsion scheme of E.coli and related organisms is called run-and-tumble. 
In Fig. 2.3a, an example trajectory of E.coli is shown. The long stretches of run-phases linked by the short parts of tumbling are clearly visible. This kind of propulsion scheme helps the organism to effectively find sources of food. In experiments, it was shown that the run-phase increases in length by a factor of two in the presence of a chemical gradient, while the tumble sections remain constant [2].

In general, the sensitivity to chemical gradients is called chemotaxis and multiple organisms invented mechanisms for following gradients of chemical attractants in one way or another. Besides the run-and-tumble of E.coli, also run-and-reverse or run-reverse-flick strategies occur in nature. Both cases outperform the run-and-tumble movement of E.coli in finding nutrient sources. Especially the run-reverse-flick of the marine bacteria Vibrio alginolyticus was found to have a threefold larger chemotactic velocity [23]. Instead of tumbling, this organism can change direction in a $90^{\circ}$ angle by flicking its single flagellum and also reverse on their own trajectory (see Fig. 2.3.).
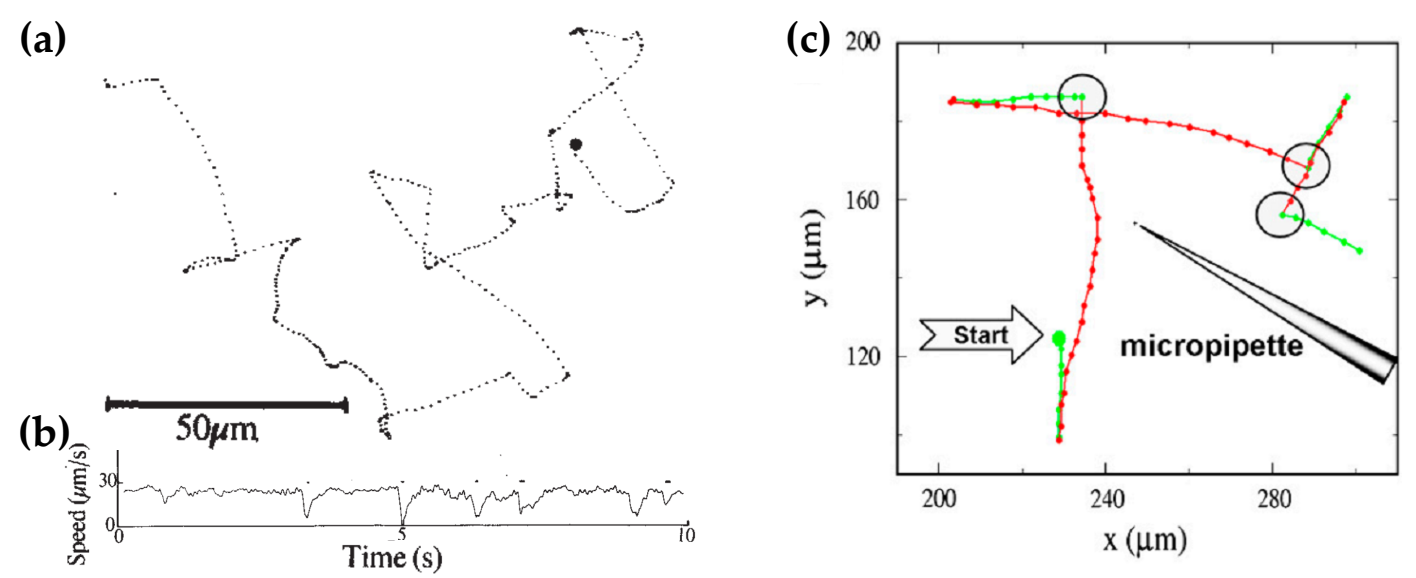

Fig. 2.3: Example trajectories of two chemotactic organisms. (a) Trajectory of E.coli wild type AW405, recorded for $29.5 \mathrm{~s}$. (b) The corresponding speed of the bacterium. Mean speed is $21.2 \mu \mathrm{m} / \mathrm{s}$ and the dots emphasize the decrease in speed due to tumbling. Taken from [2]. (c) Run-reverse-flick strategy of $V$. alginolyticus. The pipette marks the position of an emerging chemical gradient. Taken from [23].

In the vicinity of boundaries, an additional peculiarity arises in E.coli. Due to hydrodynamic interactions, circling trajectories with radii of $10-50 \mu \mathrm{m}$ are observed, depending on conditions like temperature and slip length of the interface [24]. While a first symmetry breaking is necessary for propulsion at low Reynolds numbers, a second one is needed to generate curling trajectories. In the experimental study of Lauga et al. [24], a hydrodynamical model is proposed, taking the interaction of the generated flow field with the interface into account. Briefly explained, the rotation results from viscous forces generating a torque on the swimmer's body. In the case of E.coli, the viscous drag arises from the hydrodynamic coupling of the generated flow field to the helical flagella bundle. Hence, a torque results which has to be balanced since the whole system is force free. A counter-force acting on the cell body results 
in circling trajectories. Such a curling motion of flagellates close to interfaces is also observed in other experimental studies [25-32].

However, these are by no means the earliest reports on helical motion of microswimmers. In nature, helical trajectories are observed for a number of reasons and with various proposed mechanisms. Already in 1901, Jennings [33] proposed the stabilization of trajectories as a possible mechanism of helical motion of Protozoa. Other schemes of helical motion are orientation along gradients, e.g. phototaxis [34], gravitaxis [35] or chemotaxis [36]. Crenshaw et al. provided a mathematical description of helical motion in [37], outlining that one translational degree of freedom and two rotational asymmetries are sufficient for creating helical trajectories. In simulations, a similar behaviour as compared to [24] was found by Hu et al. [38]. Inspired by E.coli, a cell propels in either counterclockwise or clockwise oriented circles, depending on the slip length of the surface. There are more examples in simulations, both, in 2D [39, 40] and 3D [41, 42], motivating the torque for curling or helical motion as a result from initial asymmetries in the system. Later in this chapter, a brief discussion is given on how such behaviour can be achieved in artificial swimmers.

So far, the mechanism of swimming and the different phenomena in dilute systems were discussed. In the following, a brief overview of denser systems and the collective phenomena will be given. Here, experimental conditions scale from oceanic dimensions to $\mathrm{cm}$ sized vessels and range down to microfluidic confinements. They all have in common that the number density of swimmers is high. Many studies were performed on bacterial cell colonies, e.g. by Goldstein et al. [43-45]. In the mentioned references, mostly the organism Bacillus subtilis was used, with an ellipsoidal body of $5 \mu \mathrm{m} \times 0.8 \mu \mathrm{m}$. Due to this anisotropy in shape, different phenomena in varying confinements of the bacteria suspensions occurred. In an unconfined reservoir in three dimensions, turbulent flow was self-generated in dense suspensions [43]. In a cylindrical confinement, spiral vortices emerge due to the nematic ordering of the bacterial bodies [44, 45]. Other studies in this field include e.g. the effect of turbulence on patches of phytoplankton [46] or the effect of mobility on rheological properties of the suspension [47, 48].

On a larger scale, upward swimming organisms like Tetrahymena pyriformis generate bioconvective patterns depending on the organism density and the height of the container [49-51]. In these comparably large flows, the whole suspension moves rather than the solution around it.

Probably the largest scale of organism turbulence is described in the Plankton paradox, stating that the high diversity of phytoplankton in the ocean is in strong conflict with all the so far known competitive exclusion principles or predator-prey dynamics. Hutchinson described this paradox for the first time in 1961 [52]. Since then, many solutions for this paradox were 
proposed without a unified picture emerging [53, 54]. Hence, artificial model systems are promising candidates to bridge the gap between nature and experiments, possibly providing solutions for these long-standing questions. In particular, results from biological systems are difficult to obtain and numerical simulations are not feasible anymore in these large scale systems.

\subsubsection{Artificial swimmer systems}

In literature, a large amount of different mechanisms of artificial propulsion are described, ranging from microrobots to Janus particles and active emulsions. In the following, freely swimming systems without the need of interfaces will be emphasized.

The first artificial swimmers were microrobots controlled by external magnetic fields as external energy sources. Dreyfus et al. [10] reported first on such a micromotor. They linked magnetic beads via DNA strands and connected these to red blood cells (see Fig. 2.4a). Thus, they gained a swimmer propelling in the flexible oar mode described by Purcell [21]. The actuation of the artificial flagellum is realized by two magnetic fields. The first field is uniform and aligns the beads along one direction. The second field oscillates along a perpendicular direction with respect to the first field and changes the shape of the flagellum. Another fabrication approach is to manufacture helices on the nanoscale and apply rotating magnetic fields [11] to follow Purcell's idea of the corkscrew swimmer (see Fig. 2.4p). The swimmers are magnetized by evaporation of a thin layer of Cobalt and controllable by magnetic fields. The propelling direction depends on the chirality of the helix and movement with speeds up to $40 \mu \mathrm{m} / \mathrm{s}$ is reported on.

(a)

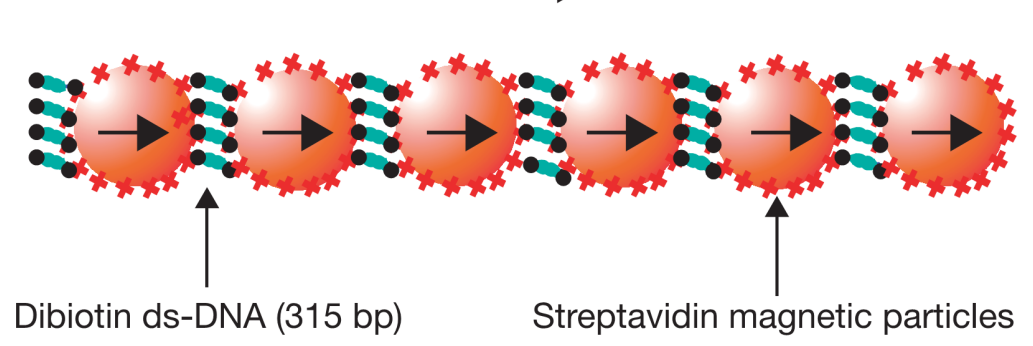

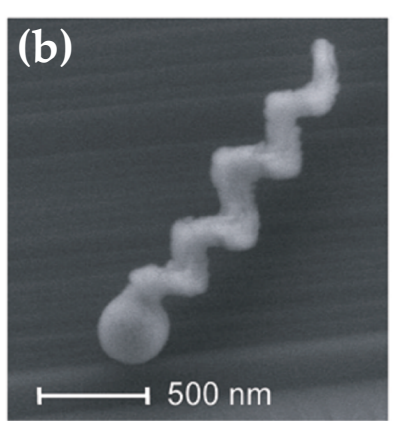

Fig. 2.4: Two exemplary microrobots utilizing the two modes of propulsion proposed by Purcell. (a) The flexible oar assembled by magnetic beads linked with DNA strands and connected to a red blood cell. Applying two magnetic fields generates propulsion. From [10]. (b) Artificial corkscrew swimmer. A rotating magnetic field triggers the propulsion of the swimmer. Taken from [11].

The most widely used artificial microswimmers are Janus particles. The term "Janus", following from the Roman god with two faces, indicates that the particle's hemispheres consist of 
two different materials. Thus, a Janus sphere has an intrinsic polar symmetry breaking. The first studies used the catalysis of $\mathrm{H}_{2} \mathrm{O}_{2}$ at a platinum interface for propulsion. Here, a flow was generated and thus, the droplet propelled in a ballistic fashion [55]. Later experimental realizations were driven by light. Here, either thermophoresis at high light intensities [56] or diffusiophoresis at low light intensities was evaluated. In the latter case a critical mixture of Lutidine/Water was used, where small increases in temperature resulted in spinodal decomposition. Introducing a Janus particle with hemispheres of different hydrophilicity resulted in propulsion [15, 57]. In all cases, ballistic motion on short time scales with velocities depending on either the concentration of $\mathrm{H}_{2} \mathrm{O}_{2}$ or the applied light intensity were observed. Intriguingly, it is also possible to fabricate different shapes of the swimmers by introducing a second symmetry breaking due to shape anisotropies. In this regard, Kümmel et al. [58, 59] reported on L-shaped particles undergoing circular swimming and also showing gravitactic behaviour.
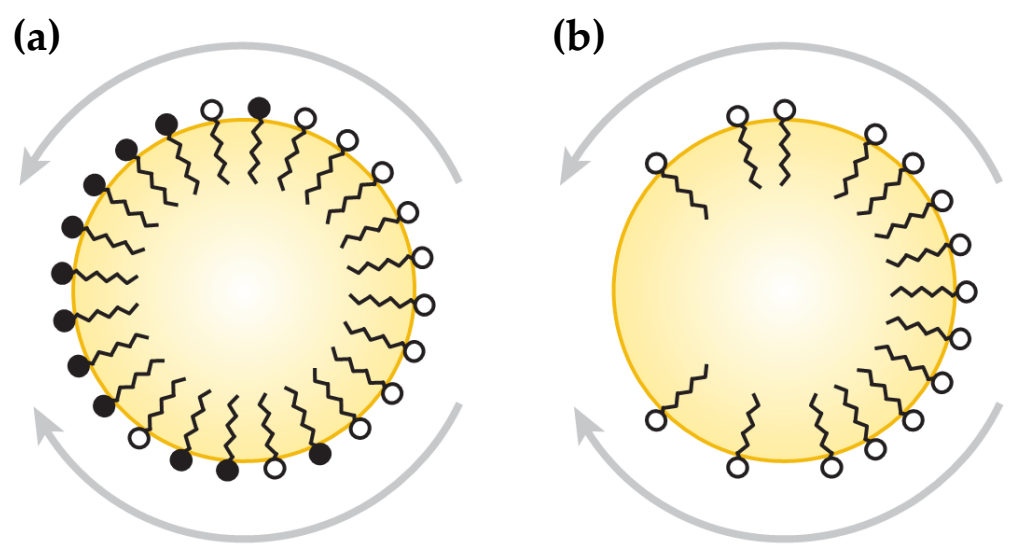

Fig. 2.5: Schematic depiction of two mechanisms for generating Marangoni stresses. (a) A chemical reaction generates an inhomogeneous interfacial tension, while maintaining a constant surface coverage. (b) Inhomogeneous coverage of one surfactant species. The grey arrows indicate the Marangoni flow from low to high interfacial tension. The droplet will be displaced in the opposite direction. Taken from [19].

The second studied group of artificial swimmers consists of active emulsions, with many different schemes proposed [19]. In most cases, complicated reactions are necessary, resulting in propulsion only on small time scales and usually with reaction products interfering with the swimmer [60-64]. A promising model of active emulsions includes systems based on Marangoni force propulsion [4, 17, 18, 65]. The latter is realized either by chemical reactions on the interface or by solubilization of the dispersed droplet medium (see Fig. 2.5). In this case, an inhomogeneity of interfacial tension results in an interface flow that generates propulsion. The system studied in this thesis consists of oil-in-water emulsions and belongs to the category of Marangoni-type swimmers utilizing solubilization mechanisms (Fig. 2.5p). In the following, the droplet material will be introduced (Sec. 2.2) and subsequently, the propulsion scheme is discussed in detail (Sec. 2.3). 


\subsection{Liquid crystal phases}

After introducing various active systems, biological and artificial, the main properties of the droplet material will be described. In the present case, the material is of liquid crystalline nature and thus, the different implications resulting from the liquid crystal anisotropy will be mentioned.

(a)

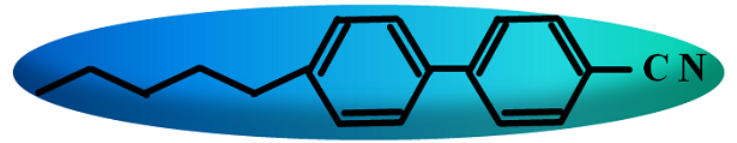

(b)

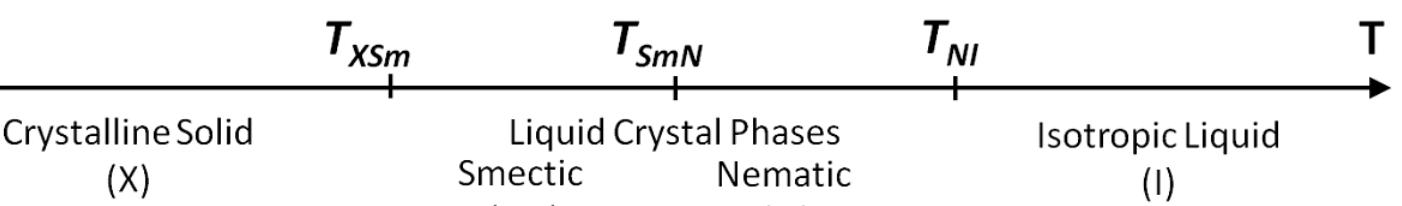

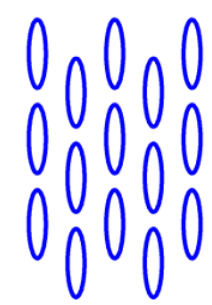

(Sm)

(N)
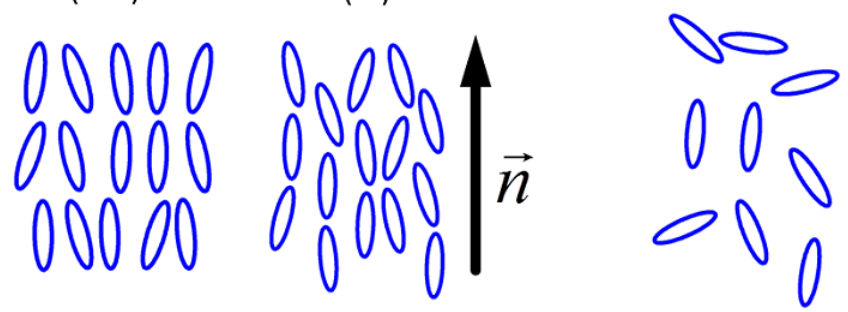

Fig. 2.6: (a) Exemplary illustration of a calamitic LC molecule, in particular of 5CB. The anisotropy in shape is readily seen. (b) Phase behaviour of a thermotropic liquid crystal. The ordering and thus the phase changes with temperature. The ordering decreases from left to right. Taken from [66].

In general, matter can take different phases: solid, liquid or gas, decreasing in the degree of ordering. In 1888, the botanist Friedrich Reinitzer observed that cholesteryl benzoate showed two melting points, changing from solid to a turbid liquid and then into a clear liquid [67]. In collaboration with the physicist Otto Lehmann, this intermediate phase was further examined. Various properties that were so far only associated with solid crystals were found and thus, the term liquid crystal (LC) was coined for these mesophases [68].

Liquid crystals are materials which, although in the liquid phase, show long-range orientational order [69]. Often, the molecules of LCs are rod like (calamitic) or disk like (discotic) in shape, resulting in anisotropic physical properties. In LC materials, one distinguishes between thermotropic and lyotropic liquid crystals. While materials of the first type switch phases by changes in temperature, the phase of the second class depend on the concentration of the monomers. LC phases can show different degrees of positional and orientational order (see Fig.2.6. 
In the case of a thermotropic LC, starting from the crystalline phase, heating the sample results in reaching the smectic phase. Here, the molecules show positional and orientational ordering, assembled in layered structures. The mean orientation of the molecules in the sample is determined by the director $\vec{n}$ and the layering is oriented parallel to $\vec{n}$. Through further heating, a second mesophase is reached. In the nematic phase, the positional ordering is lost but the orientational ordering is kept. A variant of the nematic phase is the so-called cholesteric phase, often achieved by admixture of chiral dopants to a nematic LC. Here, the orientation of the director changes in a helical fashion. In these cases, the pitch $p$, depicting the height of one complete helix turn, is an important descriptive parameter. The pitch is changing with the amount of dopant, usually in accordance to $p=\kappa / c$ with $\kappa$ in $\mu \mathrm{m} / \mathrm{wt} \%$ as a

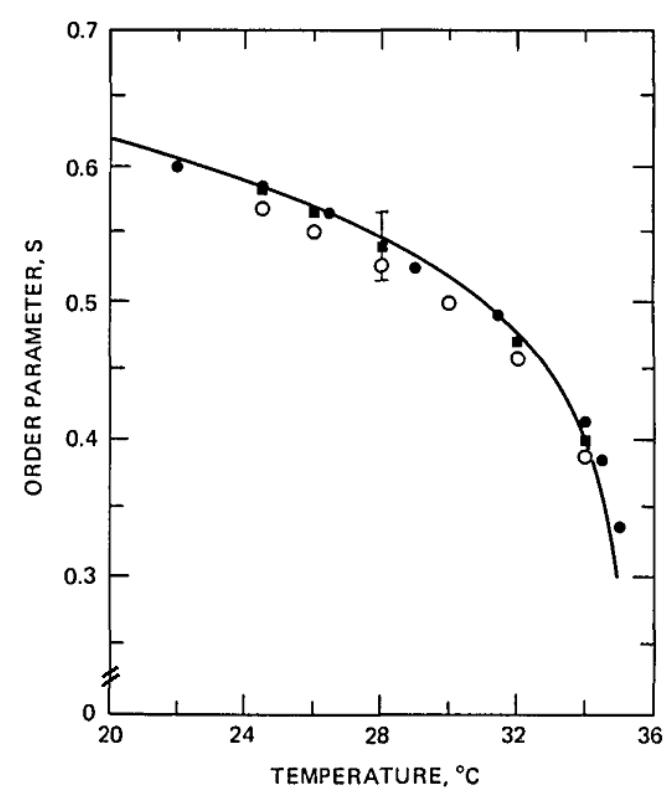

Fig. 2.7: Order parameter $S$ vs. temperature for $5 \mathrm{CB}$. The black squares correspond to results from NMR measurements. The circles correspond to calculated values. Modified in accordance to [70]. material property and $c$, the concentration in $\mathrm{wt} \%$. Heating the sample beyond the nematicto-isotropic transition erases the ordering between molecules.

The physical parameter capturing the difference of isotropic and nematic phases is the order parameter $S$. This quantity results from the correlation of the director $\vec{n}$, a vector with $\vec{n} \equiv-\vec{n}$, with the orientations of the single molecules, summed spatially and temporally along the entire sample [69]. Thus,

$$
S=\frac{1}{2}\left\langle 3 \cos ^{2} \theta-1\right\rangle
$$

with $\theta$ as the angle between the molecule's long axis and the director. The extreme cases are $S=1$ for the perfectly oriented nematic phase and $S=0$ for the isotropic phase. The order parameter varies between 0.3 and 0.8 without external influences and depends on the temperature. The transition between nematic and isotropic is a first order phase transition. In Fig. 2.7. the order parameter for $5 \mathrm{CB}$ as a function of temperature is shown.

Two major theoretical descriptions for liquid crystals have been established, the microscopical Maier-Saupe theory and the phenomenological model of de Gennes, based on the Landau theory [69]. 
The shape anisotropy of the molecules and the orientational ordering in the mesophases result in anisotropic physical properties. This has implications on the birefringence (see Sec. 4.3), the behaviour in electric and magnetic fields, the diffusion constants of tracer particles in a nematic liquid, the viscosity and other material properties [69]. Especially when it comes to nematic LCs in flows, the anisotropy of the viscosity results in interesting behaviour.

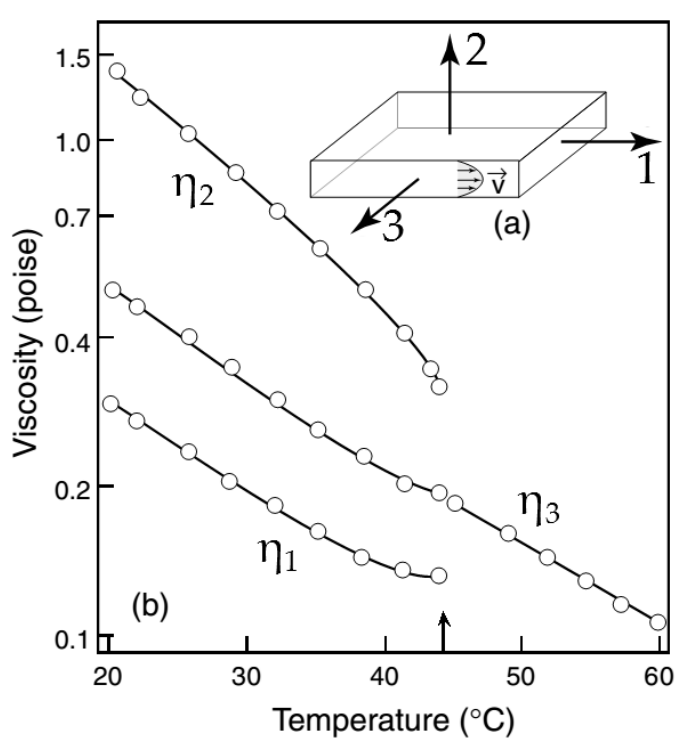

Fig. 2.8: Representation of the anisotropy of viscosity in nematic LC vs. temperature for the example of MBBA. The inset elucidates the respective director orientations as compared to the flow direction. After the nematic-to-isotropic transition, only the value for $\eta_{3}$ persists. Taken from [71].
Miesowicz first reported on nematic LCs confined between boundaries, with director orientations stabilized by strong magnetic fields and external flows applied [72]. Two relations become important, first, the flow direction as compared to the director orientation and second, the direction of the flow gradient with respect to the director orientation. This leads to a set of three viscosity coefficients (see Sec. 3.1 for values of 5CB). $\eta_{3}$ indicates that $\vec{n}$ is perpendicular to both, the flow direction and flow gradient direction (see also inset of Fig. 2.8. $\eta_{1}$ implies that the flow direction is parallel to the director $\vec{n}$, usually with the smallest contribution of the three coefficients. $\eta_{2}$ applies when the flow gradient is parallel to $\vec{n}$, obtaining the highest value of the set. With increasing temperature, all three viscosity coefficients de-
isotropic phase, the value $\eta_{3}$ is the remaining crease in magnitude. After the transition to the isotropic phase, the value $\eta_{3}$ is the remaining viscosity coefficient. One additional coefficient describes the effective viscosity for rotating molecules, the rotational viscosity $\gamma$.

Anchoring conditions and topological defects Without any external influence, the director field will gain an equilibrium orientation governed by the internal ordering of the mesophase. In the vicinity of interfaces though, molecules are biased to orient along an easy axis. This bias is called surface anchoring and is either an intrinsic property of an untreated interface or achieved by surface treatments [66]. Usually, parallel (degenerate or uniform) or perpendicular (homeotropic) alignments are distinguished. Often, mechanical rubbing or molecules are used to imprint alignment directions. For example, surface active molecules (surfactants), often used for stabilizing water/oil interfaces, can impose certain anchoring conditions [73]. The amount of external energy necessary to reorient the molecules from the 

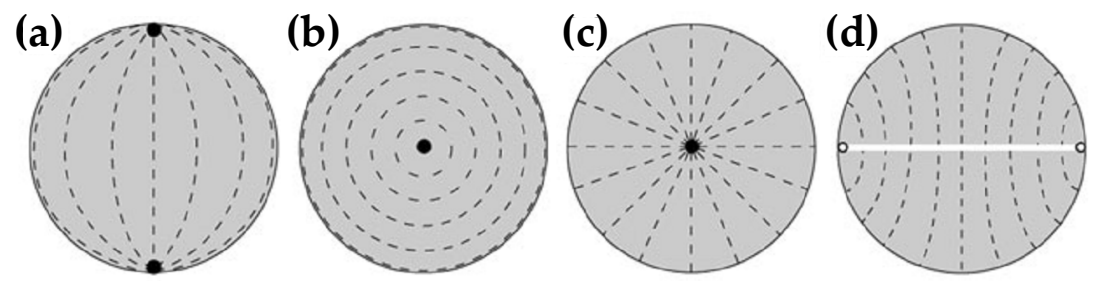

Fig. 2.9: Director structures in droplets with varying surface anchoring conditions. Dashed lines depict the local orientation of the director and black dots emphasize defects. $(a / b)$ Show droplets with planar and (c/d) show droplets with homeotropic boundary conditions. (a) Bipolar droplet with two defects (so-called boojums) at the poles of the droplet. (b) Concentric droplet with a defect in the centre and a director structure of concentric rings. (c) Radial droplet with a hyperbolic hedgehog defect located in the centre. (d) Axial droplet with an equatorial disclination ring (white line) on the surface. Adapted from [74].

equilibrium is called anchoring strength or energy. In the case of homeotropic anchoring, the energy varies between $10^{-7}$ and $10^{-3} \mathrm{~J} / \mathrm{m}^{2}$ [66].

In the specific case of LC droplets, defect structures or dislocations occur. The reason for these topologies is the interplay of the anchoring conditions and the geometric frustration due to the curved surfaces. Here, defects/dislocations describe singularities or discontinuities, similar as in solid-state crystals. Close to these defects, single molecules do not have a preferred direction of orientation. Fig. 2.9 shows different possibilities of orientational alignment in droplets, with example (c) displaying the case of the presented model system. In the later stages of the thesis, an example situation (see Sec. 5.2.5) to show the importance of the interplay of defect structures with flow is investigated.

\subsection{Propulsion of Marangoni-powered emulsions}

So far, biological and artificial model systems without details about the mathematical description have been discussed. In the following section, the model system used in this thesis is depicted in more detail, while also commenting on the mathematical description of various parameters. Therefore, swimming at low Reynolds numbers is related to micrometer-sized emulsion droplets.

The model system consists of only three ingredients - water, surfactant and oil. The basic building block for surfactants is an unpolar, hydrophobic alkyl chain of variable length and a polar, hydrophilic head group. Due to this amphiphilic structure, the molecules are well suited for stabilizing water/oil interfaces. Up to a specific concentration in a solution, called the critical micelle concentration (CMC), single molecules diffuse solely. Increasing the concentration above the $\mathrm{CMC}$ results in formation of micelles, with single molecules at the level of $\mathrm{CMC}$. The aggregation into micelles, spherical objects with single surfactant molecules lining 

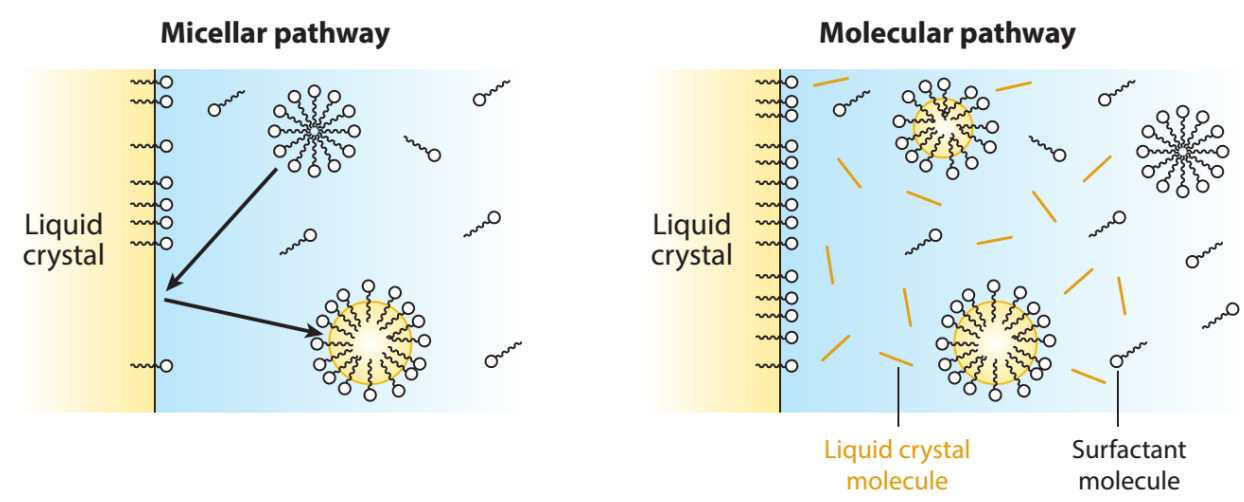

Fig. 2.10: Two pathways for solubilization of oil in surfactant solutions. (left) Micellar pathway: Micelles are filled directly at the oil/water interface. (right) Molecular pathway: Diffusion of single oil molecules into the aqueous solution where micelles collect the oil molecules. Taken from [19].

its surface, reduces the unfavourable contact of polar/unpolar parts (see Fig. 2.10] [75]. A further increase of concentration will lead to lamellar phases or to other lyotropic LC phases.

Apart from the concentration, the temperature plays an important role in the micellization of surfactants. Below a critical temperature, the Krafft-temperature, the surfactant molecules will form hydrated crystals. For homologue series, the Krafft-temperature increases for longer alkyl chains.

When an oil droplet is placed inside an aqueous surfactant solution at concentrations above the $\mathrm{CMC}$, solubilization takes place. This results in an increase of area coverage of the oil and thus, a decrease in total energy of the system. In literature, two mechanisms are proposed, the micellar and the molecular pathway, both resulting in the formation of a nanoemulsion [76]. In the first case, empty micelles attach to the oil/water interface, take up some portion of the oil and subsequently detach in a filled state (see Fig. 2.10, left). In the second case, the oil needs a finite solubility in water. For a finite distance, single oil molecules diffuse away from the interface and are incorporated into empty micelles in the continuous phase (see Fig. 2.10. right). In the theoretical framework of the latter case [4], the solubilization is accompanied by self-propulsion of the droplets, if a threshold surfactant concentration is exceeded.

As mentioned in Sect. 2.1. a symmetry breaking is necessary for self propulsion at low Reynolds numbers. Here, an advection of empty micelles at the leading edge creates this symmetry breaking and results in a stabilized Marangoni flow. The latter is generated by inhomogeneities of interfacial tension and is a mechanism for equilibrating the inhomogeneity by a flux of surfactant molecules from low to high surface tension regions. An estimation of the interfacial stresses will show that already minor changes in the equilibrium surface coverage 
can result in propulsion. Starting point is the viscous drag force [22]:

$$
F_{v i s c}=2 \pi R \eta_{c} V \frac{2+3 \mu}{1+\mu}
$$

with $\mu=\eta_{d} / \eta_{c}$ as the ratio of viscosities of the droplet medium $\eta_{d}$ and of the continuous phase $\eta_{c} . R$ and $V$ are the droplet radius and velocity, respectively. For $\mu \rightarrow \infty$, eq. (2.5) reduces to the well known Stokes drag of $F_{v i s c}=6 \pi R \eta_{c} V$ and in the limiting case of $\eta_{d} \approx \eta_{c}$, $F_{v i s c}=5 \pi R \eta_{c} V$. When taking the energy dissipation across the interface due to a moving boundary of a swimming droplet into account one finds that $F_{v i s c}$ is on the order of $4 \pi R^{2} V \nabla \gamma$. Here, $\nabla \gamma$ denotes the total variation in interfacial tension along the interface. In a damped system, this should be equal to the dissipation due to friction $F_{v i s c} V=5 \pi R \eta_{c} V^{2}$. This relation is captured by the Capillary number $C a$ with

$$
C a=\frac{\eta_{c} V}{\gamma} \approx \frac{4}{5} \frac{\nabla \gamma}{\gamma} R
$$

Following eq. (2.6), it is readily seen that already small perturbations in surfactant coverage $(\Delta \gamma=\nabla \gamma \Delta x)$ will generate velocities on the order of micrometers per second, while the droplet shape remains almost spherical [19].

The most important question is how to achieve a stable, self-sustaining gradient of surfactant coverage. In a Gedankenexperiment, let us consider an oil droplet in equilibrium with a surfactant solution. If a spontaneous motion of the droplet occurs, the surfactant coverage will be distorted in a way that surfactants accumulate at the rear end of the droplet. Mathematically, this can be described as the time dependent surface coverage $c$ of surfactants

$$
\frac{\partial c}{\partial t}=k\left(c_{0}-c\right)+\nabla\left(D_{i} \nabla c-u c\right)
$$

with $k$ as the desorption rate, $c_{0}$ as the equilibrium coverage, $D_{i}$ as the diffusion constant of the molecules and $u$ as a tangential surface velocity [4]. The induced Marangoni flow will point into the opposite direction of the spontaneous motion and will equilibrate the surfactant coverage. Thus, the droplet's propulsion is retarded and the droplet even stops. Without additional bias, the droplet therefore reverts into an equilibrium state at rest and with a homogeneous surfactant coverage. The key requirement for propulsion in Marangoni-driven systems is to orient the gradient into the propulsion direction. In this case, the advection of fresh surfactant at the leading edge has to be stronger than the diffusion of single surfactants on the interface. The parameter describing this ratio is the Péclet number Pe [22]:

$$
P e=\frac{\text { advection }}{\text { diffusion }}=\frac{L V}{D}=\frac{2 R V}{D}
$$


with a system-inherent length scale $L$, in this case $L=2 R$, and the diffusion coefficient $D$. If this ratio is above unity, propulsion can be sustained and stabilized. Parameters for the LC swimmer system are on the order of $R=25 \mu \mathrm{m}, V=10 \mu \mathrm{m} / \mathrm{s}$ and $D=10^{-10} \mathrm{~m}^{2} / \mathrm{s}$, resulting in a Péclet number of 5 . This is a reasonable approximation for propulsion and similar to the values mentioned in [77], describing a general approach of phoretically powered swimmers.
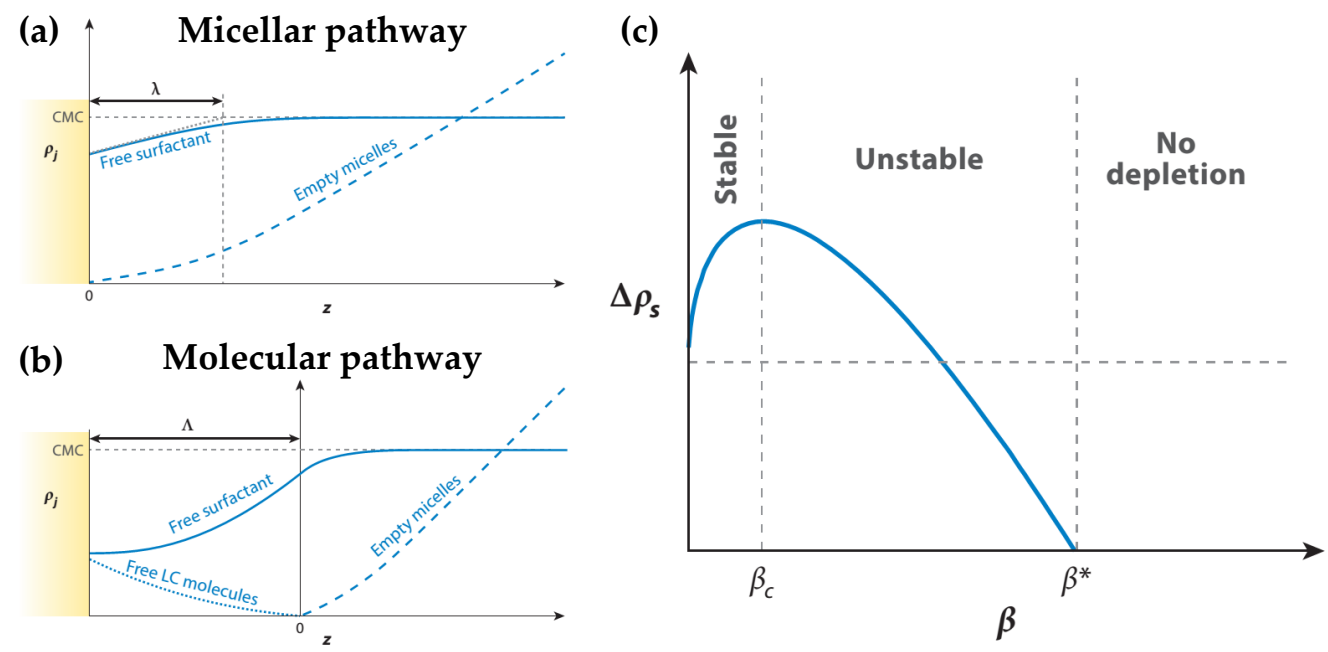

Fig. 2.11: (a) \& (b) Concentration profiles $\rho_{j}$ of the participating species $j$. The $z$-axis depicts the distance from the oil interface. (a) Micellar pathway: the interface is located at the origin with $\lambda$ as the thickness of the diffusive boundary layer. (b) Molecular pathway: the interface is located at $z<0$ with $\Lambda$ as the thickness of the reactive boundary layer. In this finite layer, all free oil molecules are incorporated into micelles. (c) Surface depletion $\Delta \rho_{s}$ of surfactants as a function of the driving parameter $\beta$. From [19].

In the case of solubilizing oil droplets, the pathway of solubilization takes a key role. The two mechanisms mentioned in Fig. 2.10 will result in swelling micelles as they take up additional free surfactants from the vicinity. Thus, different depletion patterns corresponding to the pathway of solubilization emerge. A driving parameter $\beta$ is considered, which corresponds to a gradient in empty micelle density [4, 19]. Subsequently, its influence on the depletion patterns is discussed.

In the case of the micellar pathway, empty micelles attach to the front of the droplet and detach as filled ones. Thus, they take additional free surfactant molecules directly from the droplet's interface and deplete the free surfactant density close to the leading edge of the droplet. For increasing $\beta$, this depletion is gaining in magnitude, such that the leading edge acts like a sink for free surfactant molecules. Consequently, the interfacial tension increases with stronger advection, which results in inhibition of propulsion.

In the case of the molecular pathway, a different picture emerges. Single oil molecules enter the continuous phase across the interface, diffuse up to a finite distance $\Lambda$ away from the surface and are incorporated into micelles. In this boundary layer, micelles take up additional free surfactant molecules from the continuous phase. This implies that the free surfactant 
concentration can drop significantly below the CMC. Here, the concentration is coupled to the coverage of the interface. Considering the advection of fresh molecules and empty micelles at the leading edge of the droplet, it follows from the description in [4] that $\Lambda \propto \beta^{-1}$. Thus, an increasing advection results in a decreasing depletion at the leading interface as compared to the trailing end, breaking the symmetry for this system.

The concentration profiles of the three participating species for both solubilization mechanisms are shown in Fig. 2.11. Additionally, the qualitative behaviour of the surface depletion $\Delta \rho_{s}$ versus the driving parameter $\beta$ for the molecular pathway is shown. This follows from the relation [19]

$$
\Delta \rho_{s}=c_{1} \beta^{\frac{2}{3}}+c_{2}\left(1-c_{3} \beta\right) .
$$

Droplet motion is only achieved in a region of dynamic instability that amplifies small drift velocities. Here, $d \Delta \rho_{s} / d \beta<0$, as in the case for $\beta_{c}<\beta<\beta^{*}$ with a sharp onset of motion at $\beta_{c}$. For a positive slope, the Marangoni stresses inhibit the motion $\left(\beta<\beta_{c}\right)$ and for the upper limit of $\beta$, no depletion can be achieved due to large amounts of available surfactant molecules.

For estimating the Marangoni forces achieved by this solubilization mechanism and the respective velocities, the reasoning from [18, 77] is followed. The droplet's velocity $U$ depends on the tangential surface flow and the phoretic mobility, in other words on the interaction of the particle surface with the solute. Relating these parameters and including assumptions to model the concentration profiles, the first estimation for the velocity follows as

$$
U=\frac{A M^{\prime}}{D}=\frac{\left\{3 \kappa / 4 \pi \delta^{3}\right\} \cdot\left\{k_{B} T \lambda\left[R+3 \eta_{d} \lambda^{2} / 2 \eta_{c}\right] /\left(2 \eta_{c}+3 \eta_{d}\right)\right\}}{k_{B} T / 6 \pi \eta_{c} \delta}
$$

with $\kappa$ as the dissolution rate, $\delta$ as the micelle size and $\lambda$ as an interaction length, typically in the range of nanometres. Considering the same assumptions as in [18], eq. (2.10) is reduced to

$$
U=\frac{18}{4} \frac{\kappa R}{\delta\left(2+3 \eta_{d} / \eta_{c}\right)}
$$

In Sec. 5.2.2, we will find estimates for the parameters $\kappa$ and $\delta$ and apply eq. (2.11) to the results retrieved from experiments.

\subsection{Flow fields generated by droplet swimmers}

After clarifying the mechanism of symmetry breaking and propulsion, a description of the flow field close to the droplet's interface (Chapter 9 in [22]) is given at this point. The equations are derived starting from the Stokes equation (cf. eq. (2.3)) with appropriate boundary conditions. 
In the following, the case of a spherically symmetric body immersed in a fluid at rest at infinity is treated. An external force is acting on it and the considered system is e.g. a droplet sedimenting under gravity. Generalizing this scheme to a force acting on a point at the origin, the Stokes equation offers an exact solution, called the Stokeslet [78]. Due to rotational symmetry, the derivation bases on spherical coordinates $(r, \theta, \phi)$. It is considered that a droplet of radius $R$, with its centre at the coordinate origin, is moving in the direction of the polar axis with a constant speed $V$. While the velocity component $v_{\phi}$ is zero in an axially symmetric system, the components $v_{r}$ and $v_{\theta}$ are independent of $\phi$. Solving the Stokes equation for the stream function $\Psi(r, \theta)$ results in a polynomial series. This, infinite in principle, can be truncated by taking specific boundary conditions into account, i.e. by excluding flow-field singularities. In the rest frame of the droplet, the radial and polar components of the external flow are given by [22]

$$
\begin{aligned}
& v_{r}=V \cos \theta\left[1-\frac{3+2 \mu}{2(1+\mu)} \frac{R}{r}+\frac{1}{2(1+\mu)} \frac{R^{3}}{r^{3}}\right] \\
& v_{\theta}=V \sin \theta\left[\frac{3+2 \mu}{4(1+\mu)} \frac{R}{r}+\frac{1}{4(1+\mu)} \frac{R^{3}}{r^{3}}-1\right] .
\end{aligned}
$$

At the droplet interface $(r=R)$, the radial velocity $v_{r}(R, \theta)$ follows from the boundary conditions to zero. Thus, a solely tangential flow is left with

$$
u=v_{\theta}(r=R)=-V \sin \theta \frac{\mu}{2(1+\mu)}
$$

The maximal velocity is reached at the equator with two stagnation points at the poles of the droplet (A and B in Fig. 2.12). An internal convection arises from the viscous coupling of outer and inner fluid. In the far-field $(r \gg R)$, only the $r^{-1}$ term in eq. (2.12) prevails and the characteristic flow profile of the Stokeslet is retrieved. With this algebraic decay of the flow velocity, the long range of hydrodynamic interactions is emphasized.

If droplets are purely propelled by surface stresses without external forces acting on it, a common model [79] is to derive the interfacial velocity $\mathbf{u}^{s}$ from a simple combination of Stokeslets at the droplet origin with the balanced forces. This configuration is then called a stresslet.

Moreover, for swimmers propelled with flagella either at the leading edge (squirmer type puller) or at the rear end (pusher), higher order terms in the slip velocity $\mathbf{u}^{s}$ have to be considered to describe its flow field properly [79, 80]. This results in a power series in the first derivative of the $n$-th order Legendre polynomial $P_{n}^{\prime}$ [78]. Thus, the tangential surface velocity $\mathbf{u}^{\mathrm{s}}$ is given by

$$
\mathbf{u}^{\mathcal{S}}=\sum_{n=1}^{\infty} \frac{2}{n(n+1)} B_{n}(\hat{\mathbf{e}} \cdot \hat{\mathbf{r}} \hat{\mathbf{r}}-\hat{\mathbf{e}}) P_{n}^{\prime}(\hat{\mathbf{e}} \cdot \hat{\mathbf{r}})
$$




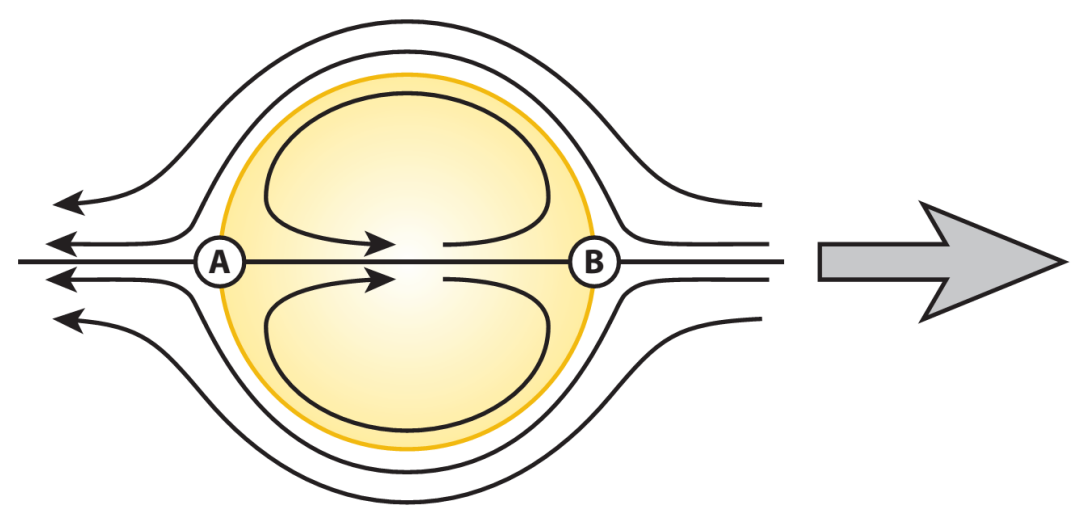

Fig. 2.12: Schematic illustration of the flow field around and inside a moving droplet in a comoving reference frame. A and B denote stagnation points, while only B is replenished with fresh surfactant. The internal convection arises due to the viscous coupling of the inner and outer phase. The grey arrow shows the propulsion direction. Taken from [19].

Here, $\hat{\mathbf{e}}$ is the squirmer's fixed swimming axis, $\hat{\mathbf{r}}$ is a unit vector pointing from the particle centre to the surface and $B_{n}$ is the $n$-th mode of the surface squirming velocity. However, recent numerical studies showed that it is sufficient to consider only the first two orders $(n<3)$ of the power series of the interface velocity to achieve reasonable accuracy when approximating the hydrodynamic flow profiles of flagellated swimmers [81, 82]. With the aforementioned assumptions, the polar velocity component in spherical coordinates follows from eq. (2.14) as

$$
u_{\theta}=B_{1} \sin \theta+\left(B_{2} / 2\right) \sin 2 \theta
$$

with $\theta$ as the polar angle [82]. The speed $V$ of the squirmer depends solely on $B_{1}$ and is given by $V=2 B_{1} / 3$, while the second mode $B_{2}$ determines the strength of the stresslet. Thus, a parameter $\beta^{\prime}$ is defined to relate the second mode of squirming to the first mode of squirming, resulting in $\beta^{\prime}=B_{2} / B_{1}$. Note that a primed expression $\beta^{\prime}$ is used that varies from literature, since the expression $\beta$ is already exploited in a different context (see Sec. 2.3).

However, it is possible to distinguish the different squirmer classes by $\beta^{\prime}$. The neutral squirmer is given for $\beta^{\prime}=B_{2}=0$, the pusher relates to $\beta^{\prime}<0$ and the puller is described by $\beta^{\prime}>0$. In Fig. 2.13, the two force dipole squirmers with their respective biological equivalents are shown. Additionally, the surface velocities $u_{\theta}$ for all three squirmer classes are depicted [80, 82].

So far, the flow field was mainly considered in the vicinity of the surface. The far field contribution can be derived from a multipole expansion of the Stokes equation. Thus, the velocity field results from a power series in $r^{-1}$. In eq. (2.12), the $r^{-1}$ far-field dependency for a Stokeslet is already described, with an external force acting on the sphere. In the terms of a 

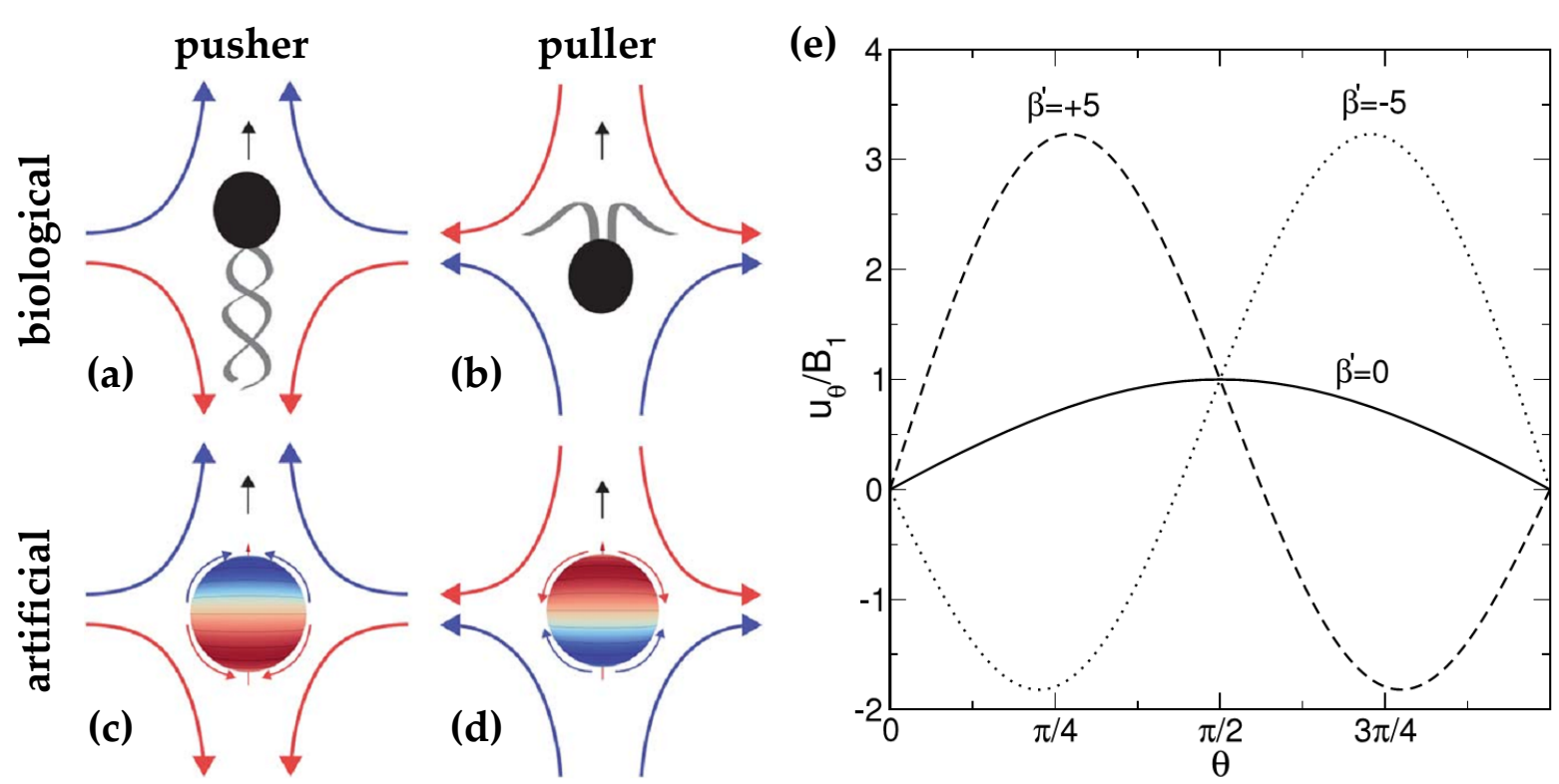

Fig. 2.13: (a) - (d) Schematic representation of the propulsion mechanism and flow profiles of squirmers for biological (top row) and artificial (bottom row) examples. (a) \& (c) Quantities for a pusher and (b) $\&$ (d) for a puller type squirmer. The propulsion direction is indicated by the black arrows. Taken from [80]. (e) Polar velocity on the surface of the three squirmer classes. Taken from [82].

squirmer, which is force and torque free and generates a driving on its own, one can derive a dipolar flow velocity in the far-field that decays with $r^{-2}$. This is similar to electrostatic charges. And indeed, the creation of a stresslet by two Stokeslets with forces pointing into opposite directions resembles two charges with opposite signs. In such a configuration, the $r^{-1}$ contributions of each of the Stokeslets in the far field cancel each other and only higher order terms remain.

With the technique of Particle Image Velocimetry, it is possible to map the flow profile around the squirmers and thus, to determine the propulsion mode (see Sec.4.6).

\subsection{Brownian motion \& mean squared displacement}

Hitherto, active propulsion mechanisms have been discussed. For micron-sized particles, an additional contribution has to be taken into account, the Brownian motion. Brownian motion describes the intrinsic behaviour of small particles to move randomly when in equilibrium with a fluid at a finite temperature $T$. The movement results from the interaction of the particle with the solvent molecules. If an additional active component superposes with the random motion, a new term is coined, the active Brownian motion. In the following, a brief description of the underlying physics and mathematical description is given. 
In the early 19th century, the Scottish botanist Robert Brown reported first on the random motion of pollen grains floating in water, excluding currents in the gradually evaporating fluid as sources for this movement [83]. The mathematical description was not found until the beginning of the 20th century when two scientists, quite simultaneously, started to solve the problem. The well known paper of Albert Einstein covered the problem first, considering the osmotic pressure as the driving force [84, 85]. The relation of osmotic pressure forces to the resistance of the fluid due to friction delivered the famous equation for the diffusion coefficient $D$ :

$$
D=\frac{k_{B} T}{m \gamma}
$$

with $k_{B}$, the Boltzmann constant and $\gamma$, the friction coefficient. For spherical particles with radius $R$ in a fluid, eq. 2.16 results in the Stokes-Einstein-equation with the additional relation of $m \gamma=6 \pi \eta R$. Marian van Smoluchowski tackled the same problem with a different ansatz and formulated a kinetic model with only minor deviations from Einstein's result despite of the various assumptions made [86]. A stochastic approach by Paul Langevin bridged the gap between Einstein's and Smoluchowski's contributions, starting with a Newtonian ansatz and thus, taking the forces acting on the particle into account. The 1D case is given by [87]

$$
m \dot{v}_{x}=-m \gamma v_{x}+\xi(t) .
$$

The first term on the r.h.s. describes the velocity-dependent dissipative force resulting from friction, the second term introduces the interaction of the particle with the solvent molecules. Following the assumptions of Gaussian white noise, $\xi$ has to fulfil the conditions

$$
\langle\xi(t)\rangle=0,\left\langle\xi_{i}(t) \xi_{j}\left(t^{\prime}\right)\right\rangle=\delta_{i j} \delta\left(t-t^{\prime}\right)
$$

Calculating the mean of eq. 2.17) and taking the equipartition theorem into account, $\left\langle v_{x}^{2}\right\rangle=$ $\frac{k_{B} T}{m}$, a differential equation emerges. Applying the initial condition $x(0)=0$, the well known expression for the mean squared displacement (MSD) is derived:

$$
\left\langle\Delta x^{2}(t)\right\rangle=\left\langle x\left(t_{0}+t\right)-x\left(t_{0}\right)\right\rangle_{t_{0}}^{2}=\frac{2 k_{B} T}{m \gamma}\left\{t-\frac{1}{\gamma}[1-\exp (\gamma t)]\right\} .
$$

The prefactor is the diffusion constant that has already been mentioned in eq. 2.16). Two regimes, depending on the time scale, arise. The short time scale limit for $t \ll 1 / \gamma$ is neglected, since it has only an impact on the timescale of nanoseconds. In the limit for $t \gg 1 / \gamma$, the MSD approaches 


$$
\left\langle\Delta x^{2}(t)\right\rangle=2 D t-\frac{2 D}{\gamma} \approx 2 D t
$$

As a consequence, the one-dimensional MSD for a purely Brownian-driven particle increases linearly with time, with a slope of $2 D$. Expanding this result to three dimensions yields $\left\langle\Delta \mathbf{r}^{2}(t)\right\rangle=6 D t$.

As already mentioned, not only translational diffusion has an impact on micron-sized particles but also rotational diffusion. In analogy to $\left\langle\Delta x^{2}(t)\right\rangle$, the mean angular displacement is given by [88]

$$
\left\langle\Delta \theta^{2}(t)\right\rangle=2 D_{r} t
$$

with $D_{r}=\frac{k_{B} T}{8 \pi \eta R^{3}}$.

In the case of an active swimmer, an additional term for the propulsion force $\left(f_{\text {prop }}\right)$ has to be added to the Langevin equation (see eq. (2.17)). We cover the case for two dimensions via

$$
m \dot{\mathbf{v}}=-m \gamma \mathbf{v}+f_{\text {prop }}+\xi(t)
$$

In order to obtain a MSD solution for this ansatz, a new time constant is introduced. Depending on the rotational diffusion $\tau_{r}=1 / D_{r}$, the propulsion direction is reoriented and thus, the persistence of the swimmer is defined. These assumptions lead to [55]

$$
\left\langle\Delta \mathbf{r}^{2}(t)\right\rangle=4 D t+\frac{v^{2} \tau_{r}^{2}}{2}\left[\frac{2 t}{\tau_{r}}+\exp \left(-\frac{2 t}{\tau_{r}}\right)-1\right]
$$

with the limiting cases:

$$
\begin{array}{ll}
\left\langle\Delta \mathbf{r}^{2}(t)\right\rangle=4 D t+v^{2} t^{2} \quad \text { for } t \ll \tau_{r}, \\
\left\langle\Delta \mathbf{r}^{2}(t)\right\rangle=\left(4 D+v^{2} \tau_{r}\right) t \quad \text { for } t \gg \tau_{r} .
\end{array}
$$

The MSD of Platinum-coated Janus particles (Fig. 2.14 will be used as an example to illustrate the different time scales. For short time scales (red line), the ballistic propulsion outweighs the rotational diffusion and adds quadratically to the MSD. The characteristic persistence length for the ballistic propulsion follows from $L=v \tau_{r}$. For long times (green curve), the diffusive contribution results in a randomization of the propulsion directions, also called Random Walk, with an effective diffusion constant of $D_{\text {eff }}=D+v^{2} \tau_{r} / 4$. 


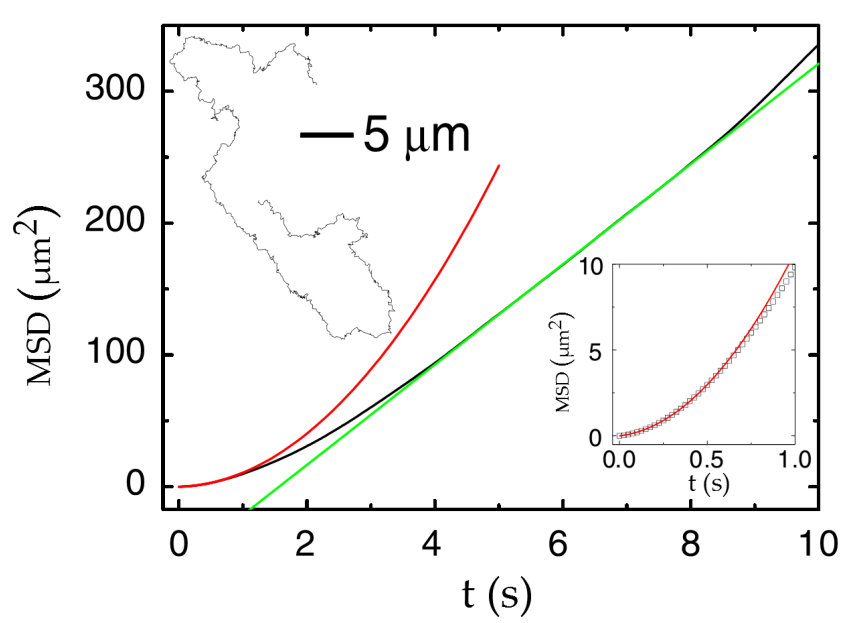

Fig. 2.14: $\mathrm{MSD}$ of $1.6 \mu \mathrm{m}$ sized Platinum coated Janus particles, catalysing the reaction of $\mathrm{H}_{2} \mathrm{O}_{2}$. Data for $10 \% \mathrm{H}_{2} \mathrm{O}_{2}$. The red curve emphasizes the short time regime, the green line the long time regime. The inset magnifies the course for times below $1 \mathrm{~s}$. The analysis provides a reorientation time of $\tau_{r}=3.9 \mathrm{~s}$. Adapted from [55].

As an estimation, the diffusion constants in eqs. (2.16) and 2.21) are calculated for three different diameters of 15, 50 and $120 \mu \mathrm{m}$ for LC swimmers in aqueous surfactant solutions (see Tab. 2.1).

Tab. 2.1: Theoretical diffusion constants and persistence times. Calculated for $T=293 \mathrm{~K}$ and $\eta_{\mathrm{H}_{2} \mathrm{O}}=1 \mathrm{mPa} \cdot \mathrm{s}$ for three different particle radii in the micron range.

\begin{tabular}{cccc}
\hline $\mathrm{R}(\mu \mathrm{m})$ & $\mathrm{D}\left(\mu \mathrm{m}^{2} / \mathrm{s}\right)$ & $\mathrm{D}_{r}\left(\right.$ radians $\left.^{2} / \mathrm{s}\right)$ & $\tau_{r}(\mathrm{~s})$ \\
7.5 & $2.9 \times 10^{-2}$ & $3.8 \times 10^{-4}$ & $2.6 \times 10^{3}$ \\
25 & $8.6 \times 10^{-3}$ & $1.0 \times 10^{-5}$ & $9.7 \times 10^{4}$ \\
60 & $3.6 \times 10^{-3}$ & $7.4 \times 10^{-7}$ & $1.3 \times 10^{6}$ \\
\hline
\end{tabular}

As can be seen from the theoretical values, the rotational diffusion for spherical particles with these diameters is negligible. However, other mechanisms in artificial propulsion might lead to a reorientation of the swimmers, see e.g. Sections 5.2.2 and 5.2.4.

At this point, an additional analysis parameter is introduced, the velocity autocorrelation $C(t)$. This is a measure to determine the persistence length of a swimmer's trajectory. The relation is given by [89]

$$
C(t)=\left\langle\frac{\mathbf{v}\left(t_{0}+t\right) \cdot \mathbf{v}\left(t_{0}\right)}{\left|\mathbf{v}\left(t_{0}+t\right)\right|\left|\mathbf{v}\left(t_{0}\right)\right|}\right\rangle_{t_{0}}
$$

For moving particles, the correlation will exponentially decay over time with the persistence time $\tau_{p} \approx \tau_{r}$ as the decay constant. 



\section{Materials}

This section introduces the employed materials, namely the LC utilized as droplet material and the ionic surfactants used for stabilizing the interfaces and to generate propulsion by Marangoni flows. The parameters given below will be applied to quantify the experimental results (see Sec. 5).

\subsection{Liquid crystals}

In experiments presented in this thesis, mainly droplets consisting of the calamitic, thermotropic liquid crystal 4-pentyl-4'-cyanobiphenyl (5CB) are used. 5CB is popular in experimental studies since it is nematic at room-temperature and furthermore a low-cost material with appropriate viscosities for microfluidic applications. Synthon Chemicals (Germany) distributes this material that can be used without further purification. The rod-like LC molecule consists of two linked phenyl rings as a centre part, attached to a nitrile group (CN) on one end and a carbon alkyl chain $\left(\mathrm{C}_{5} \mathrm{H}_{11}\right)$ on the other. Selected physical properties of $5 \mathrm{CB}$ are listed in Tab. 3.1.

Tab. 3.1: Properties of 5CB [90, 91]. The schematic representation of the molecule is taken from [92].

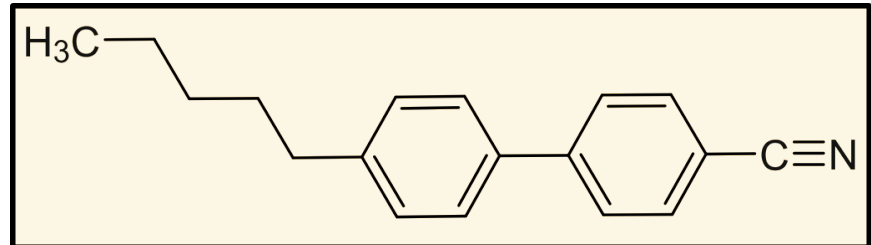

\begin{tabular}{|c|c|c|c|c|}
\hline \multicolumn{5}{|c|}{ Thermodynamic properties } \\
\hline $\mathrm{M}(\mathrm{g} / \mathrm{mol})$ & \multicolumn{2}{|c|}{$\rho\left(\mathrm{kg} / \mathrm{m}^{3}\right)$} & $\mathrm{T}_{X N}\left({ }^{\circ} \mathrm{C}\right)$ & $\mathrm{T}_{N I}\left({ }^{\circ} \mathrm{C}\right)$ \\
\hline 249.359 & \multicolumn{2}{|c|}{1002.4} & 24 & 35.3 \\
\hline \multicolumn{5}{|c|}{ Optical properties } \\
\hline $\mathrm{T}\left({ }^{\circ} \mathrm{C}\right)$ & \multicolumn{2}{|c|}{$n_{\|}$} & $n_{\perp}$ & $\Delta n$ \\
\hline 25 & \multicolumn{2}{|c|}{1.727} & 1.539 & 0.188 \\
\hline 36 & \multicolumn{2}{|c|}{1.65} & 1.65 & 0 \\
\hline \multicolumn{5}{|c|}{ Viscosity coefficients } \\
\hline $\mathrm{T}\left({ }^{\circ} \mathrm{C}\right)$ & $\eta_{1}(\mathrm{mPa} \cdot \mathrm{s})$ & $\eta_{2}(\mathrm{mPa} \cdot \mathrm{s})$ & $\eta_{3}(\mathrm{mPa} \cdot \mathrm{s})$ & $\gamma_{1}(\mathrm{mPa} \cdot \mathrm{s})$ \\
\hline 25 & 22 & 123 & 43 & 77 \\
\hline 36 & 26 & 26 & 26 & - \\
\hline
\end{tabular}


The numerical classification of CB liquid crystals is derived from the length of the alkyl chain. The molecule shape results in highly anisotropic properties and a strong polarity. 5CB, as a small homologue in this class, only undergoes the nematic-to-isotropic transition, lacking more ordered phases. At the transition temperature $T_{N I} \approx 34.5^{\circ} \mathrm{C}$, the local ordering diminishes and the cloudy appearance of the material changes to transparent. When decreasing $T$ below $T_{X N} \approx 22.4^{\circ} \mathrm{C}, 5 \mathrm{CB}$ crystallizes.

In a few experiments in Sec. 5, instead of temperature as a trigger of the isotropic transition, admixture of a second oil is used. The respective oil is 1-Bromopentadecane (BPD, $\mathrm{CH}_{3}\left(\mathrm{CH}_{2}\right)_{13} \mathrm{CH}_{2} \mathrm{Br}$ ) purchased from Sigma Aldrich (USA). The molecular weight is $M=293.31$ $\mathrm{g} \mathrm{mol}^{-1}$ and the density is $\rho=1005 \mathrm{~kg} \mathrm{~m}^{-3}\left(25^{\circ} \mathrm{C}\right)$, close to the density of $5 \mathrm{CB}$. Admixture of $\approx 8 \mathrm{vol} \%$ is sufficient to create isotropic $5 \mathrm{CB} / \mathrm{BPD}$ droplets without inhibiting the self-propulsion. Pure 1-Bromopentadecane droplets show no propulsion.

A second kind of material is introduced to change the director structure of the nematic $5 \mathrm{CB}$ to a chiral LC (see Sec. 5.4.1). By admixture of R811 or $S 811$ (in particular: (R)/(S)-Octan-2yl 4-((4-(hexyloxy)benzoyl)oxy)benzoate), right- or left-handed helical director structures are generated with the pitch $p$ depending on the weight fraction of the dopant. The chemicals are purchased from Merck KGaA (Germany). For these two dopants, the $\kappa$-parameter equals $10 \mu \mathrm{m} / \mathrm{wt} \%$ [93]. Concentrations between 0.05 and $4 \mathrm{wt} \%$ are used, resulting in pitches of $200 \mu \mathrm{m}$ down to $2.5 \mu \mathrm{m}$.

\subsection{Ionic surfactants}

Surface-active agents play a major role in active emulsions to stabilize oil/water interfaces (see Sec. 2.3). Without this effect, the swimming droplets would not remain spherical, but coalesce or wet the reservoir boundaries. Furthermore, solubilization processes as shown in Fig. 2.10 are impossible without micelles being present.

In the present thesis, mainly aqueous solutions of the cationic surfactant Tetradecyltrimethylammonium Bromide (TTAB) are used. In the case of TTAB, an alkyl chain consists of 14 carbon atoms and is attached to a charged ammonium head group with a negative bromine ion as the counterion. For some experiments, the higher homologue CTAB (16 carbon atoms) or the anionic surfactant Sodium Dodecyl Sulfate (SDS) are employed. Some chemical properties of the surfactants are listed in Table 3.2. For TTAB, concentrations between 0.1 and $25 \mathrm{wt} \%$ are applied. All surfactants are purchased from Sigma Aldrich and used without further purification. Non-ionic surfactants are not utilized, as the more complicated interactions of micelles and droplet interfaces are not included in the model presented in Sec. 2.3. In case of non-charged micelles, the micellar pathway and inverted micelles inside the oil droplet are possible. 
Tab. 3.2: Properties of ionic surfactants. Data obtained from [75]. Molecular structures from [94-96].

\begin{tabular}{|c|c|c|c|}
\hline Amphiphile & molecular structure & $\mathrm{CMC}$ in $\mathrm{H}_{2} \mathrm{O}(\mathrm{wt} \%)$ & $\begin{array}{c}\text { Krafft Temperature } \\
\left({ }^{\circ} \mathrm{C}\right)\end{array}$ \\
\hline TTAB $\left(\mathrm{C}_{14} \mathrm{TAB}\right)$ & $\begin{array}{r}\mathrm{CH}_{3} \mathrm{Br} \\
\mathrm{CH}_{3}\left(\mathrm{CH}_{2}\right)_{12} \mathrm{CH}_{2}-\mathrm{N}^{+}-\mathrm{CH}_{3} \\
\stackrel{!}{\mathrm{CH}_{3}}\end{array}$ & 0.13 & - \\
\hline CTAB $\left(\mathrm{C}_{16} \mathrm{TAB}\right)$ & $\begin{array}{c}\underset{\mathrm{C}}{\mathrm{I}} \mathrm{H}_{3} \mathrm{Br}^{-} \\
\mathrm{H}_{3} \mathrm{C}\left(\mathrm{H}_{2} \mathrm{C}\right)_{15}-\underset{N^{+}-\mathrm{CH}_{3}}{\stackrel{\mathrm{C}}{\mathrm{C}} \mathrm{H}_{3}} \\
\mathrm{H}_{3}\end{array}$ & 0.035 & 25 \\
\hline SDS & $\mathrm{CH}_{3}\left(\mathrm{CH}_{2}\right)_{10} \mathrm{CH}_{2} \mathrm{O}-\mathrm{N}_{\mathrm{O}}^{\mathrm{O}}-\mathrm{ONa}$ & 0.187 & 16 \\
\hline
\end{tabular}

An extensive study of the properties of TTAB micelles can be found in Haramagatti's PhD thesis [97]. Here, Small Angle Neutron Scattering (SANS) was used to obtain the geometrical parameters of empty micelles like the aggregation number $N_{a g g}$, meaning the number of molecules assembled in a single micelle, and the shape of the micelle defined by the length of the three axes $a, b$ and $c$. This resulted in the insight that, at low surfactant concentrations close to the $\mathrm{CMC}$, the micelles are nearly spherical, while with increasing concentrations, the shape changes to prolate ellipsoidal. The reason is that the micelles grow along the major axis $c$ and are restricted in growth along the minor axes $a$ and $b$. Furthermore, shape changes of swelling micelles caused by an uptake of a solute at constant surfactant concentration $c_{s}$ was studied in [98]. The results of both studies are individually shown in Fig. 33.1 a, b and combined in Fig. 3.1k.

The data from [97] is converted from $\mathrm{mM}$ to $\mathrm{wt} \%$ to match the notation of this thesis (blue graphs in Fig. 3.1F). This study covers the range of $c_{s}$ between 0.85 and $22.5 \mathrm{wt} \%$ TTAB and provides the SANS spectra as well as values for $N_{a g g}$ and $c$. In the case of the study in [98], only the SANS spectra are given. In these measurements, the surfactant concentration was kept constant at $c_{S}=25 \mathrm{wt} \%$, while the concentration of the solute $5 \mathrm{CB}$ was changed in the range from 0 to $3 \mathrm{wt} \%$. For a comparison, the maximum value of the spectra $Q_{\max }$ are obtained and subsequently, the approximate solution given in [97] is followed to derive the values for the long axis $c$ and the aggregation number $N_{a g g}$.

Both studies provide data for $N_{a g g}$ and $c$ in the high surfactant range. However, a slight deviation in the data is observed. This might be due to the difference in the aqueous solvent for the surfactant, heavy water $\mathrm{D}_{2} \mathrm{O}$ [97] versus light water $\mathrm{H}_{2} \mathrm{O}$ [98]. For completeness, the equations with the appropriate approximations derived in [97] are given. Here, the aggregation number $N_{a g g}$ is assumed to be 

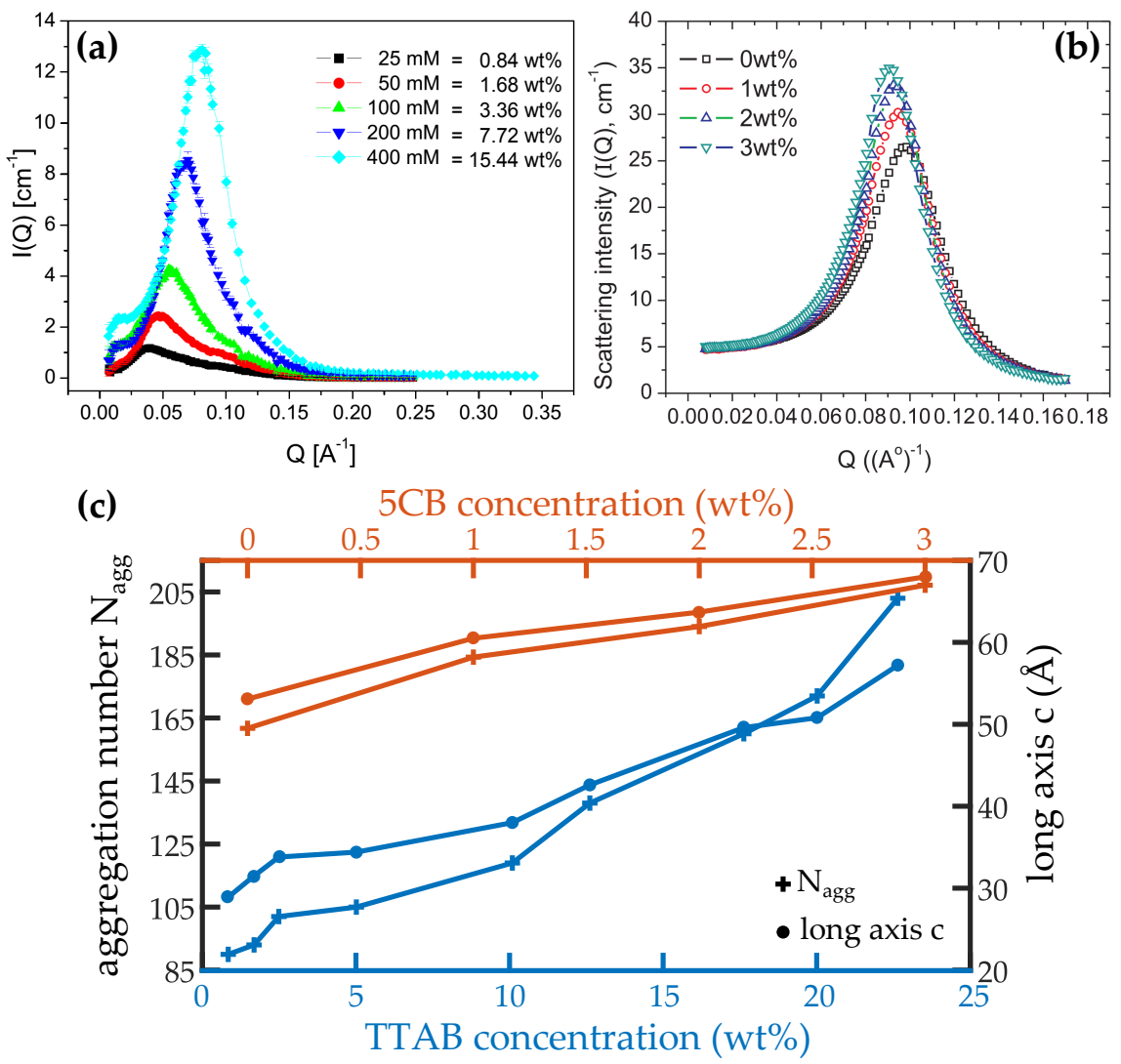

Fig. 3.1: Collected values for TTAB micelles. (a) SANS measurements of TTAB $/ \mathrm{D}_{2} \mathrm{O}$ solutions with varying surfactant concentration $c_{s}$. Adapted from [97. (b) SANS measurement of TTAB micelles in $\mathrm{H}_{2} \mathrm{O}$ with differing amount of solute $5 \mathrm{CB}$. In this case, $c_{S}$ is constant at $25 \mathrm{wt} \%$ TTAB and the concentration of $5 \mathrm{CB}$ changes between 0 and $3 \mathrm{wt} \%$. Adapted from [98]. (c) Combined data from [97, 98]. The symbols distinguish the two calculated quantities. Cross symbols depict the aggregation number $N_{\text {agg }}$ and dots mark the values for the long axis $c$ of the micelles. The data from [97] is shown in blue, with changing surfactant concentration $c_{S}$ and no solute present. The data at constant surfactant concentration $c_{s}=25 \mathrm{wt} \%$ TTAB with increasing solute concentration is depicted in orange [98].

$$
N_{a g g}=\frac{\left(c_{s}-\mathrm{CMC}\right) N_{L}}{f Q_{\max }^{3}}
$$

with $c_{s}$ and $\mathrm{CMC}$ in $\mathrm{mM}, N_{L}$ as the Avogadro constant and $f$ as a factor depending on the local structure of the micellar solution, in this case $f=2.88 \times 10^{-3}$ [97]. The long axis $c$ of the micelle is calculated from the volume of an ellipsoid $V=4 / 3 \pi a b c$. The volume $V$ is approximated by $V=V_{0} N_{a g g}$, with $V_{0}=550 \AA^{3}$ as the effective volume of a single surfactant. Hence,

$$
c=\frac{3 V_{0} N_{a g g}}{4 \pi a b}
$$

with $a=b \approx 20 \AA$. 


\section{Methods}

In this chapter, the methods used during the course of experiments will be introduced. This elucidation covers a large range from microfabrication techniques and microfluidic applications to optical methods necessary for data acquisition and the post processing of data, e.g. droplet tracking and particle image velocimetry. The introduction of light sheet microscopy covers a brief review of geometrical optics and beam width measurements. Furthermore, a characterization of droplet production in terms of diameter vs. flow rates and polydispersity is given.

\subsection{Microfabrication}

\subsubsection{Photolithography}

The preparation of the microfluidic chips follows a top-down approach using soft-lithography. The first step is to prepare a mask for the master silicon wafer via photolithography [100]. Depending on the type of the resist, either positive or negative, the areas illuminated need to be chosen appropriately. In this case, the photoresist is SU-8, a negative photoresist. Thus, areas which subsequently serve as microfluidic channels are illuminated. Masks are created in a 2D AutoCad application and printed as a high resolution emulsion film by an external company (128k DPI, JD Photo-Tools, UK).

Following Fig. 4.1, the first step done in house is to cover the silicon wafer with photoresist by spin coating. The height of the photoresist layer defines the height of the resultant channels. To control this, the SU-8 photoresist is used

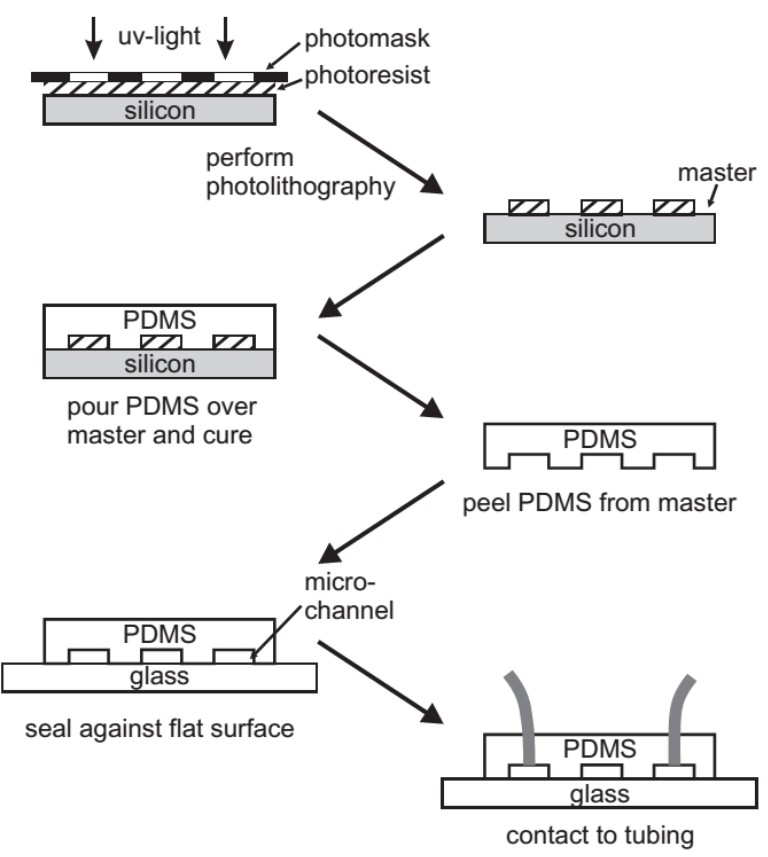

Fig. 4.1: Schematic illustration of general steps for fabricating microchannels, using photolithography and PDMS as a polymer. Taken from [99]. in different viscosities and the parameters for the spin coating are chosen accordingly. Illumination of the wafer with UV light through the mask will crosslink the photoresist under 
transparent areas on the mask. The removal of these areas during the developmental processing of the wafer is therefore prevented. This step finalizes the so-called master. Afterwards, the master is used as a mould for Polydimethylsiloxan (PDMS).

The PDMS (Sylgard 184, Dow Corning, USA) is composed of two separate ingredients, a polymer and a cross-linking agent. Mixing these two parts in a 10:1 weight ratio produces the final polymer. Degassing the mixture, pouring it over the wafer and curing it at $75^{\circ} \mathrm{C}$ for $2 \mathrm{~h}$ yields the PDMS replica. Subsequently, the layer is peeled off, cut into single pieces and inlets for the tubing are punched.

To guarantee a tight seal between PDMS and glass after contact bonding, a preceding plasma cleaning step is necessary. Therefore, a mixture of air/oxygen is used to terminate the $\mathrm{OH}^{-}$groups rendering a covalent bonding between PDMS and glass. Connecting the inlets and outlets with Teflon tubing finalizes the microfluidic channel.

PDMS is originally hydrophobic, by two hours after plasma treatment at the latest. Thus, an additional surface treatment step is necessary when using a flow focusing device for the production of oil-in-water emulsions [101]. After activation of the surfaces by a 1:1 volumetric mixture of $\mathrm{H}_{2} \mathrm{O}_{2} / \mathrm{HCl}$, the channel is filled with a silanization solution $\left(\left(\mathrm{C}_{2} \mathrm{H}_{2} \mathrm{O}\right)_{n} \mathrm{C}_{7} \mathrm{H}_{18} \mathrm{O}_{4} \mathrm{Si}\right)$ for $30 \mathrm{~min}$. Afterwards, the channel is rinsed with milli-Q water. The surfaces are now covered by a hydrophilic layer of a few nanometre thickness.

For the flow focusing devices, channel widths between $20-100 \mu \mathrm{m}$ and heights of 45 or $95 \mu \mathrm{m}$ are used to generate droplets of various diameters.

\subsubsection{Experimental reservoirs}

The fabrication procedure mentioned in Sec. 4.1.1 is valid for all microfluidic channels, independently of the use for droplet production or as experimental chambers. However, there are further ways of producing experimental reservoirs. Microfluidic geometries can e.g. be made directly from SU-8. In this case, the polarity of the mask is inverted and the photoresist is spincoated directly onto a glass slide of $50 \times 75 \mathrm{~mm}^{2}$. The process follows Sec. 4.1.1 without the need of manufacturing a PDMS replica. The major advantages in this procedure are the adjustment of the layer thickness in the $\mu \mathrm{m}$ range and the variability in reservoir geometries due to the versatile photolithography. On the other hand, a drawback of these reservoirs is the height limitation $(10-130 \mu \mathrm{m})$ due to the different thermal expansion rates of glass and photoresist. Anyhow, these reservoirs are used directly after fabrication and after filling them with liquid, they are sealed by cover slides to prevent evaporation.

A further fabrication procedure is applied for chambers with heights exceeding $200 \mu \mathrm{m}$. Here, a steel mould is produced by milling and subsequently filled with PDMS. Due to the me- 
chanical manufacturing constraints the geometries of the reservoirs are limited and thus, only circular geometries with differing diameters are produced in this manner. The resultant PDMS chambers are directly used without a surface treatment and closed by a cover glass after being filled with liquid.

\subsection{Droplet production in microfluidics}

This section is dedicated to the recipes for producing monodisperse droplets. In the past years, different emulsification procedures have been described, ranging from sonication, shear induced emulsification [102], shearing fluids between plates [103] and pushing liquid through a two-dimensional array of pores [104] to microfluidic techniques, characterized by throughput and polydispersity of the emulsion. In the methods mentioned above, the monodispersity increases, while the throughput decreases. Thus, microfluidic methods are incredibly useful for the precise control of droplet sizes, while the throughput is often secondary. Usually, the microfluidic chips are manufactured in PDMS, allowing to either produce water-in-oil or oilin-water emulsions, since the wettability of the surfaces can be changed accordingly [101]. Either volumetric pumps with fixed volume flow rates $Q$ or pressure-driven pumps are used for applying flow in the channel.

In general, different schemes for droplet production in microfluidics have been proposed [99, 105-108] and for the emulsification of LCs specifically [109, 110]. For the experiments in this thesis, the flow focusing geometry is chosen [107, 111, 112]. In this geometrical arrangement, two immiscible fluids meet at a cross junction of four channels. Here, two opposite channels are filled with the continuous phase fluid, the third channel is filled with the fluid of the dispersed phase and the generated emulsion leaves the junction in the fourth direction (s. Fig. 4.3).
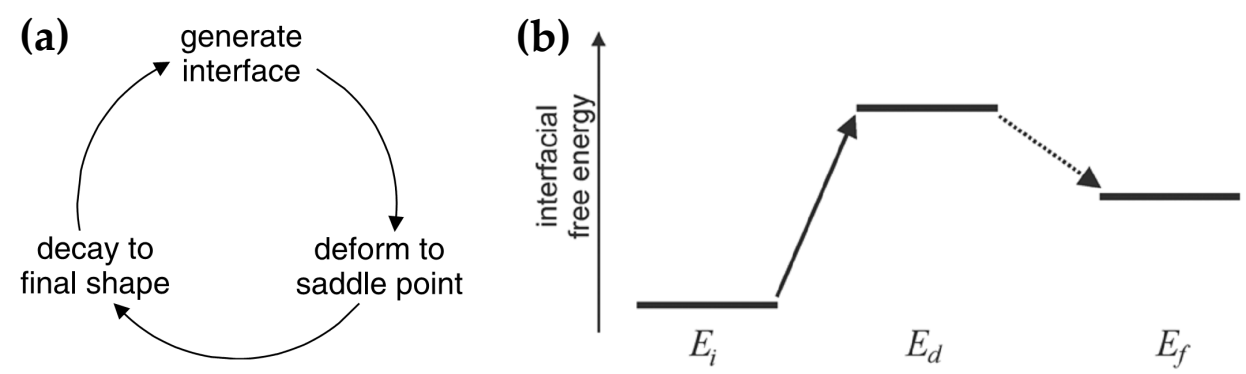

Fig. 4.2: Schematic of droplet generation, in terms of universal steps and interfacial free energy. (a) Depicts the basic steps of a droplet generation device. (b) Shows the evolution of droplet generation in terms of interfacial free energy. Starting from two individual fluids $\left(E_{i}\right)$, generating an interface is necessary. Subsequently, the interface is deformed across the saddle point $\left(E_{d}\right)$. Finally, it decays into dispersed droplets inside a continuous liquid $\left(E_{f}\right)$. Both images taken from [99]. 


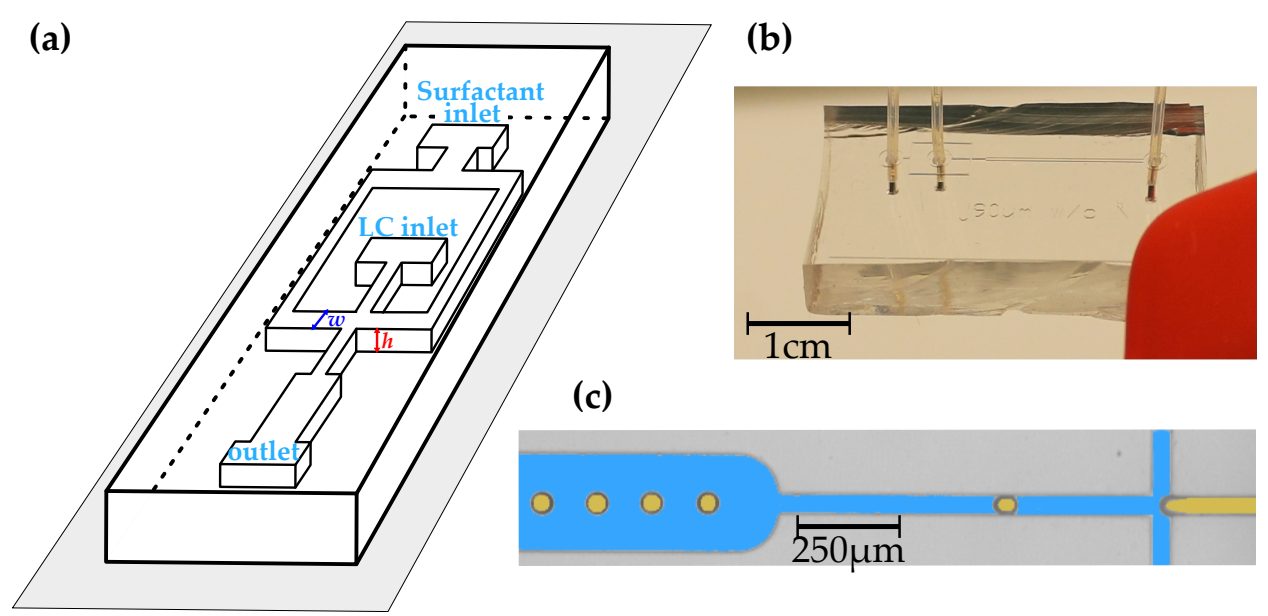

Fig. 4.3: (a) Schematic representation of a microfluidic flow-focusing device with the width $w$ (blue) and height $h$ (red) of the channel. (b) An image of a PDMS device with inserted tubing before contact bonding to a glass plate. (c) Coloured micrograph of droplet production in the dripping mode recorded with a high speed camera. The continuous surfactant solution is depicted in blue and the dispersed LC phase in yellow.

Basically, the droplet generation follows the scheme depicted in Fig. $4.2 \mathrm{a}$ with the related energy levels shown in (b). At the meeting point, an interface is generated between the two immiscible fluids. This interface first deforms and increases the interfacial energy of the system until it spontaneously decreases beyond the saddle point $\left(E_{d}\right)$. Subsequently, the system relaxes into the final state $\left(E_{f}\right)$ with a higher energy as compared to the initial energy level of two individual fluids $\left(E_{i}\right)$ [99]. Following Young's equation, these energy levels depend on the interfacial tensions between the fluids and the channel surfaces [113]. To describe the process in other words, droplets are generated when the viscous drag force of the continuous liquid exceeds the capillary forces of the dispersed liquid.

In the flow-focusing device, different modes of droplet production are obtained by adjusting the ratio of the flow rates. The so-called dripping mode is achieved for small flow rates of both, dispersed and continuous fluid phase. Here, droplet pinch-off occurs when the dispersed phase blocks the outlet channel, the pressure in the constriction increases and the continuous fluid pushes the two interfaces together. When increasing the continuous flow rate above a threshold, droplets are generated by a Rayleigh-Plateau instability. The latter occurs downstream from the cross junction and results in smaller droplets as compared to the dripping mode. When the dispersed-phase flow is further increased, such that the dispersed fluid fills the outlet chamber, the emergent behaviour is specified as tubing and results in large droplets close to the size of the collection chamber [114].

Dimensionless numbers are obtained from the principal parameters of the two immiscible fluids, in particular the Capillary number and the Reynolds number. The Capillary number is defined as $C a_{i}=\eta_{i} u_{i} / \gamma$ with $\eta_{i}$ as the fluid viscosity, $u_{i}=Q_{i} /(w \times h)$ (see Fig. 4.3a) as the 
(a)

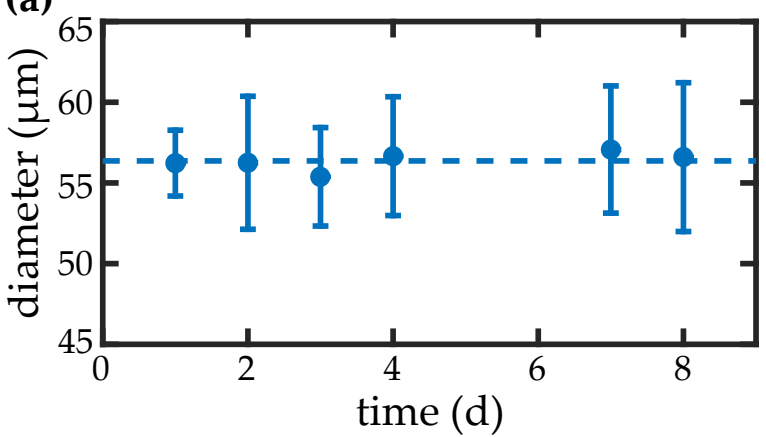

(b)

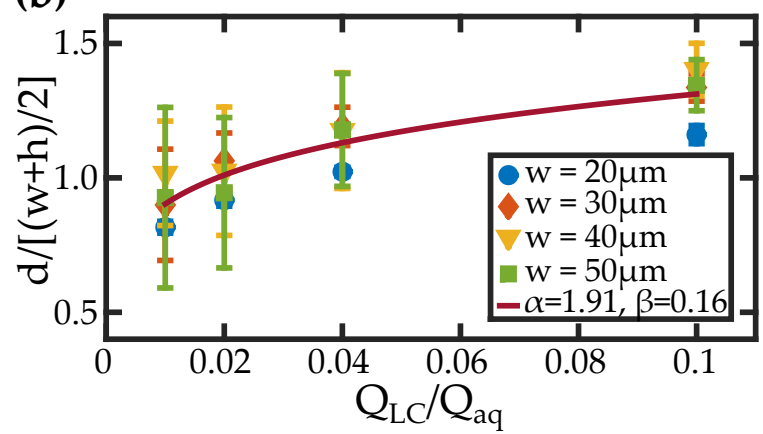

Fig. 4.4: (a) Stability of droplet diameter over time. The horizontal line emphasizes the mean value of the data points. (b) Normalized droplet size vs. flow-rate ratio. $Q_{L C}=10 \mu \mathrm{L} / \mathrm{h}$ was kept constant. The fit follows $d /[(w+h) / 2]=\alpha \cdot\left(Q_{L C} / Q_{a q}\right)^{\beta}$.

fluid velocity and $\gamma$, the interfacial tension [115]. For co-flow geometries, values of $C a_{\text {crit }} \approx 0.1$ are experimentally found for the dripping regime [116, 117]. The Reynolds number is given by $R e_{i}=\rho_{i} Q_{i} /\left(\eta_{i} h\right)$ with $\rho_{i}$, the density of liquid $i$ and $h$, the height of the square microchannel. Usually, $R e \ll 1$ applies and thus, inertial effects play no role [117]. Empirically, a relation for the droplet size ratio $d / w$ to the flow-rate ratio was found to be $d / w=\alpha\left(Q_{d} / Q_{c}\right)^{\beta}$ with $\alpha \geq 1$ and $\beta \leq 1$ [109, 115, 118, 119]. As an example, the values for the liquid crystal droplet production in silicon oil are cited with $\alpha=3.14$ and $\beta=0.65$ [109]. Additionally, Hamlington et al. reported on different regimes for stable droplet production for both, the nematic and the isotropic phase.

A disadvantage of using PDMS as a polymer is that some solvents swell PDMS and that there is a finite evaporation of water in long-term measurements. Both effects are negligible in our cases, since the experimental times are rather small and swelling was not observed for the liquids in use [66, 120].

An overview of the calibration data obtained in the experimental droplet production is given in Fig. 4.4. In this case, flow focusing devices with a variable junction width $(20-50 \mu \mathrm{m})$ but constant height of $45 \mu \mathrm{m}$ were used. The utilized fluids are 5CB as the dispersed phase and an aqueous solution of $0.1 \mathrm{wt} \% \mathrm{TTAB}$ as the continuous phase. The concentration of surfactant is high enough to prevent coalescence of the droplets, but below the CMC to rule out solubilization of the droplets over time. Flow rates $Q$ between 5 and $1000 \mu \mathrm{L} / \mathrm{h}$ are applied by syringe pumps (neMESYS, Cetoni, Germany) resulting in flow velocities between 0.15 $300 \mathrm{~mm} / \mathrm{s}$.

Fig. 4.4 a shows the stability of the emulsion obtained in a $40 \mu \mathrm{m}$ wide cross junction at a flowrate ratio of $Q_{L C} / Q_{a q}=0.1$ with $Q_{L C}=10 \mu \mathrm{L} / \mathrm{h}$. It can be seen that, within the experimental errors, there is no apparent decrease in droplet size due to i.e. solubilization over a period of several days. 
Fig. 4.4p shows the normalized droplet diameter vs. the flow-rate ratio. Here, the diameter is normalized by the mean value of channel width and height since rectangular cross sections are used in the current designs. The fit parameters $\alpha$ and $\beta$ are obtained and show reasonable values as compared to the literature, in particular $\alpha=1.91$ and $\beta=0.16$. The occurrence of slightly higher polydispersity than reported on for other flow-focusing devices is related to the anisotropic viscosity of the liquid crystal.

\subsection{Polarized Optical Microscopy}

In this section, the technique of Polarized Optical Microscopy (POM) is introduced. Besides regular brightfield microscopy, this is the main technique for observing liquid crystalline samples. Additionally, various parameters that are not obtainable by other visual means, especially the director configuration, can be determined with this approach. The present technique exploits the anisotropic shape of LC molecules through the resulting birefringence.

When considering a uniaxial LC molecule, the anisotropy generates two different refractive indices with respect to the molecule axis. The index component $n_{\|}$along the optical axis coincides with the director orientation, whereas $n_{\perp}$ is perpendicular to the latter. 5CB is an optically positive material, meaning that $n_{\|}>n_{\perp}$. An incoming ray of light is split into two orthogonal components, the extraordinary and the ordinary ray. The refractive index $n_{o}$ that influences the ordinary ray corresponds to $n_{\perp}$. The refractive index $n_{e}$ depends on the angle $\phi$, defined by the direction of the incident light and the orientation of the optical axis [121]:

$$
n_{e}=\frac{n_{\|} n_{\perp}}{\sqrt{n_{\|}^{2} \cos ^{2} \phi+n_{\perp}^{2} \sin ^{2} \phi}} .
$$

The two refractive indices already indicate the modulation of an incident ray of light. The optical anisotropy will result in different propagation speeds of the orthogonal beam components and thus, they exhibit a phase difference $\delta$ after propagation through the material. Taking the vacuum wavelength $\lambda$ and the sample thickness $d$ into account, $\delta$ is given by

$$
\delta=\frac{2 \pi}{\lambda}\left(n_{e}-n_{0}\right) d
$$

with $\left(n_{e}-n_{0}\right)=\Delta n$ as the optical birefringence of a material. The phase difference imposed on formerly linear polarized light by birefringent materials generates elliptically polarized light. In POM, these effects are exploited, as depicted in Fig. 4.5. Two polarizers are inserted into a regular brightfield transmission microscope, the polarizer and the analyzer. With the polarizer, one linear direction of the initially unpolarized light is chosen. After passing 
through the sample, the outcoming beam is probed by the analyzer, usually enclosing an angle of $90^{\circ}$ with the polarizer. If the material is not birefringent, the polarization of the beam remains unchanged and no light will pass the analyzer. If birefringence occurs, the intensity $I$ of the transmitted beam depends on [121]

$$
I=I_{0} \sin ^{2}(2 \varphi) \sin ^{2}\left(\frac{\delta}{2}\right)
$$

with $I_{0}$ as the intensity after the polarizer and $\varphi$ as the angle between the analyzer and the projection of the optical axis into the sample plane.

\section{Polarized Light Microscope Configuration}

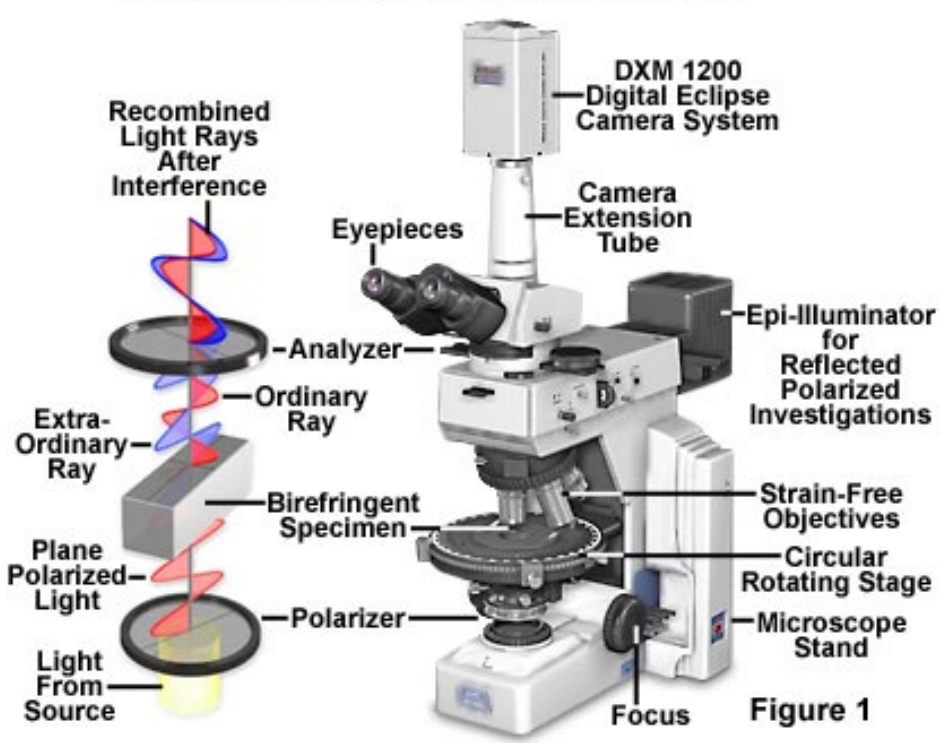

Fig. 4.5: (left) Schematic representation of polarization direction change due to a birefringent specimen. (right) Example of a Nikon Eclipse LV100. Taken from [122].
For the swimmer system, POM is especially helpful to determine the location of the point defect that is generated by the homeotropic anchoring on the droplet surface [73, 123]. This is investigated by using an Eclipse LV100 (Nikon, Japan) POM equipped with a commercial digital camera (EOS 600d, Canon, Japan). Fig. 4.5 shows a schematic representation of the microscope. Both, polarizer and analyzer, are rotary mounted with an accuracy of $\pi / 180=1^{\circ}$. Multiple objective lenses with differing magnification in the range of $2 \times-100 \times$ are accessible. The numerical aperture changes between 0.5 for air and 1.4 for oil immersion objective lenses.

Another helpful device in POM is a $530 \mathrm{~nm}$ retardation $(\lambda)$-plate, which encloses a $45^{\circ}$ angle with the polarizer orientation and is placed before the analyzer. With this plate, the interference colours of the phase shifted beam components are visualized and thus, the director orientation in nematic fields can be determined. Figs. 4.6a and (b) depict a self-propelling droplet with homeotropic anchoring, in the case of (a) imaged with crossed polarizers and in the case of (b) with an additional retardation plate. Fig. 4.6. illustrates how the interference colours appearing in (b) are related to the orientation of the liquid crystal molecules. 


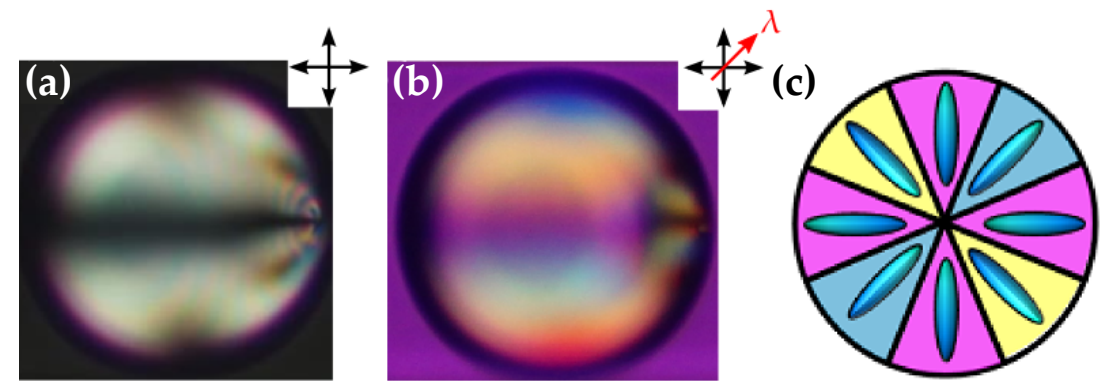

Fig. 4.6: Polarized images of $5 \mathrm{CB}$ droplets propelling in an aqueous surfactant solution. The surfactant imposes homeotropic anchoring. (a) Droplet under crossed polarizers (black arrows) and (b) with additional retardation $(\lambda)$-plate (red arrow). (c) Schematic representation of emerging interference colours for differing director orientations when a retardation plate is applied. (c) Taken from [66].

\subsection{Light-Sheet Microscopy}

Two-dimensional data sets are easily recorded by brightfield microscopy but mapping samples in three dimensions requires more advanced techniques. Different approaches for a full 3D reconstruction of experimental volumes include block-face imaging, tomography techniques (MRI \& CT), stereoscopic cameras or confocal laser-scanning techniques (CLSM). The tomography techniques are expensive and pose furthermore certain requirements on the sample, while confocal laser-scanning techniques exhibit other disadvantages. The basis of CLSM is confocal fluorescence microscopy, meaning that a laser beam is focused into the sample and that the signal is detected through a pinhole. Despite of the high resolution and contrast achievable, the method is found to be slow, it introduces photobleaching onto sections of the sample surrounding the focal spot and has only a limited penetration depth [124]. Instead, a technique from developmental biology is introduced, related to CLSM but without the mentioned disadvantages, called Light Sheet Fluorescence Microscopy (LSFM) [125, 126]. The main difference is the sample illumination by a thin laser sheet instead of a focused beam. Through sectioning the sample into planes instead of points or voxels, the photobleaching is reduced and the scanning speed is increased.

Even though there is a plethora of mentioned setup variations, the basic concept is always the same. The illumination unit consists of a laser, beam expander and a cylindrical lens. The latter generates a thin laser sheet focused only in one direction, leaving the other direction unaltered. The detection unit is oriented perpendicular to the illumination plane and consists of an objective lens, a tube lens, a fluorescence filter and a CCD camera [124]. The scanning of the sample is realized either by rotating the sample stage [126] or by moving the laser sheet and keeping the sample fixed [127]. With this basic setup, 2D images are recorded and processing yields three dimensional reconstructions. The lateral resolution in LSFM is determined, simi- 


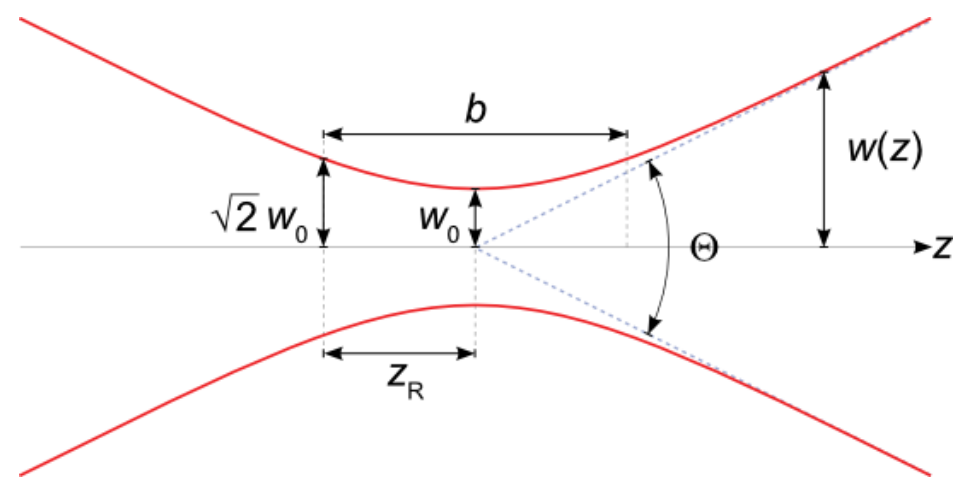

Fig. 4.7: Schematic illustration of a focused Gaussian beam. Depicted is the beam width $w(z)$ vs. the axial distance $z$ with the beam waist $w_{0}$ at $z=0$, the Rayleigh length $z_{R}$ and the confocal parameter $b=2 z_{R}$. Taken from [132].

lar as in brightfield microscopy, by the numerical aperture $N A$ of the detection objective lens and the fluorescence wavelength $\lambda$ of the fluorophore [124]. For a theoretical approximation, the resolution in the lateral plane is given by [128]

$$
r_{\text {lateral }}=\frac{\lambda}{2 N A}
$$

In the axial direction, the width of the light sheet defines the resolution, since the depth-offield (DOF) of a low NA objective is comparably larger. Theoretically, the DOF results from [128-130]:

$$
r_{\text {axial }}=\frac{\lambda n}{N A^{2}}+\frac{n e}{M N A}
$$

with $M$, the magnification of the objective and $e$, the smallest distance that can be resolved by the detector placed in the image plane of the objective. This is similar to the pixel size when using a CCD camera. Note that the light sheet undergoes divergence across the field-of-view (FOV), such that the confocal parameter $b$ plays a crucial role to describe the behaviour across the sample [131]. In the following, the theoretical description of the Gaussian beam waist of the laser sheet is given (see Fig. 4.7).

When a laser beam is focused by a lens, it obtains a minimum size $d$ at a finite distance $z$ from the lens, obeying the diffraction limit $d \geq \lambda$. Closer examination shows a Gaussian intensity distribution perpendicular to the propagation direction. A beam width $w(z)$ can be attributed to every distance $z$, with the convention that $z=0$ for the position of the beam waist $w_{0}$. The evolution of the beam width $w(z)$ is described by [133] 


$$
w(z)=w_{0} \sqrt{1+\left(\frac{\lambda z}{\pi w_{0}^{2}}\right)^{2}}
$$

Over a distance of $z_{R}$, the beam width increases to $\sqrt{2} w_{0} . z_{R}$ is called Rayleigh length and is defined as

$$
z_{R}=\frac{\pi w_{0}^{2}}{\lambda}
$$

Finally, from the Rayleigh length, the confocal parameter follows as $b=2 z_{R}$, defining the region with beam properties suitable for LSFM.

For the fluorescence signal, a dye is introduced into the nematic matrix, the fluorophore NileRed. This dye is used in various LC studies since it is not only fully soluble in the LC phase but also aligns with the director axis [134, 135]. Fig. 4.8 shows the molecular structure and the fluorescence characteristics of NileRed.

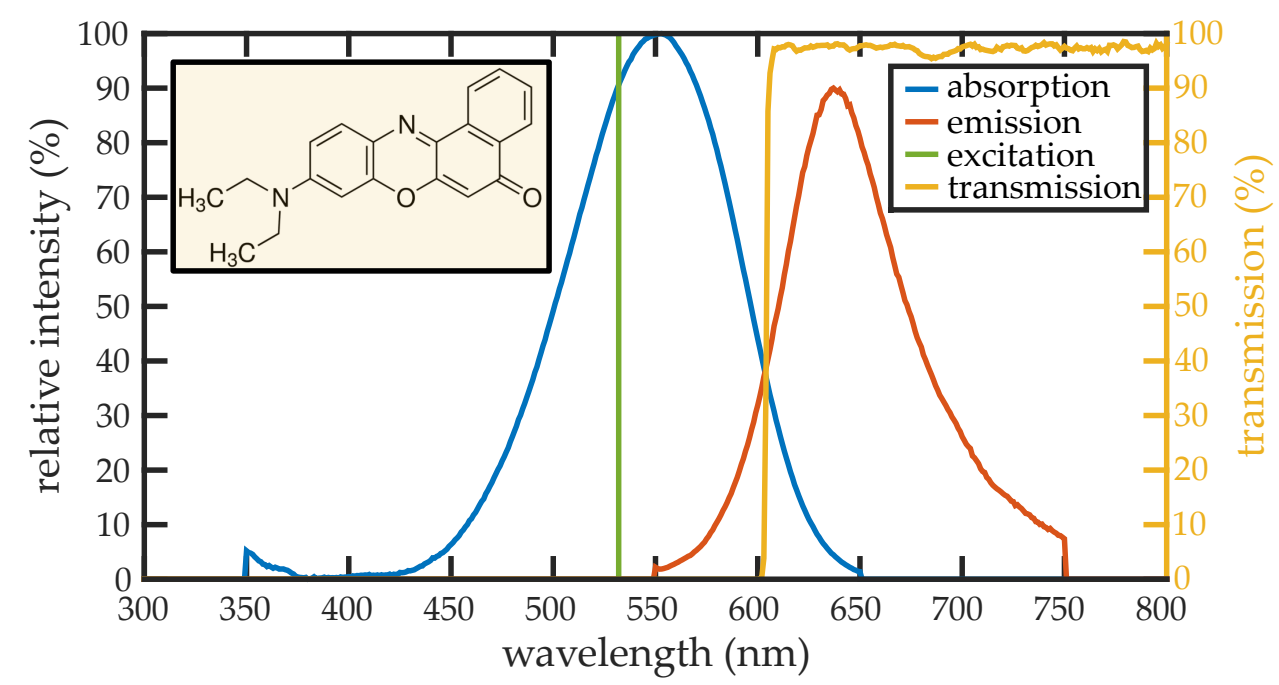

Fig. 4.8: Characteristics of the fluorophore NileRed. (Inset) Representation of the molecular structure [136]. The graph depicts the absorption (blue) and emission (orange) spectra of NileRed [137]. Additionally, the excitation wavelength of the laser diode (green) and the transmission of the long pass filter in front of the CCD camera (yellow) [138] are given. Thus, the full fluorescence boundaries of the light-sheet setup are presented.

Since the theoretical background is given above, the focus is now set on the technical realization of a light sheet setup and the sample preparation. In Fig. 4.9 , a schematic illustration of the in-house built setup is given. The setup and basic calibration was realized in a Masters thesis by Gunnar Klös [139] and has subsequently been improved. The most common application of LSFM in literature is the imaging of cell tissue [126, 127]. However, our goal is the scanning of large volumes of swimmer suspensions. 


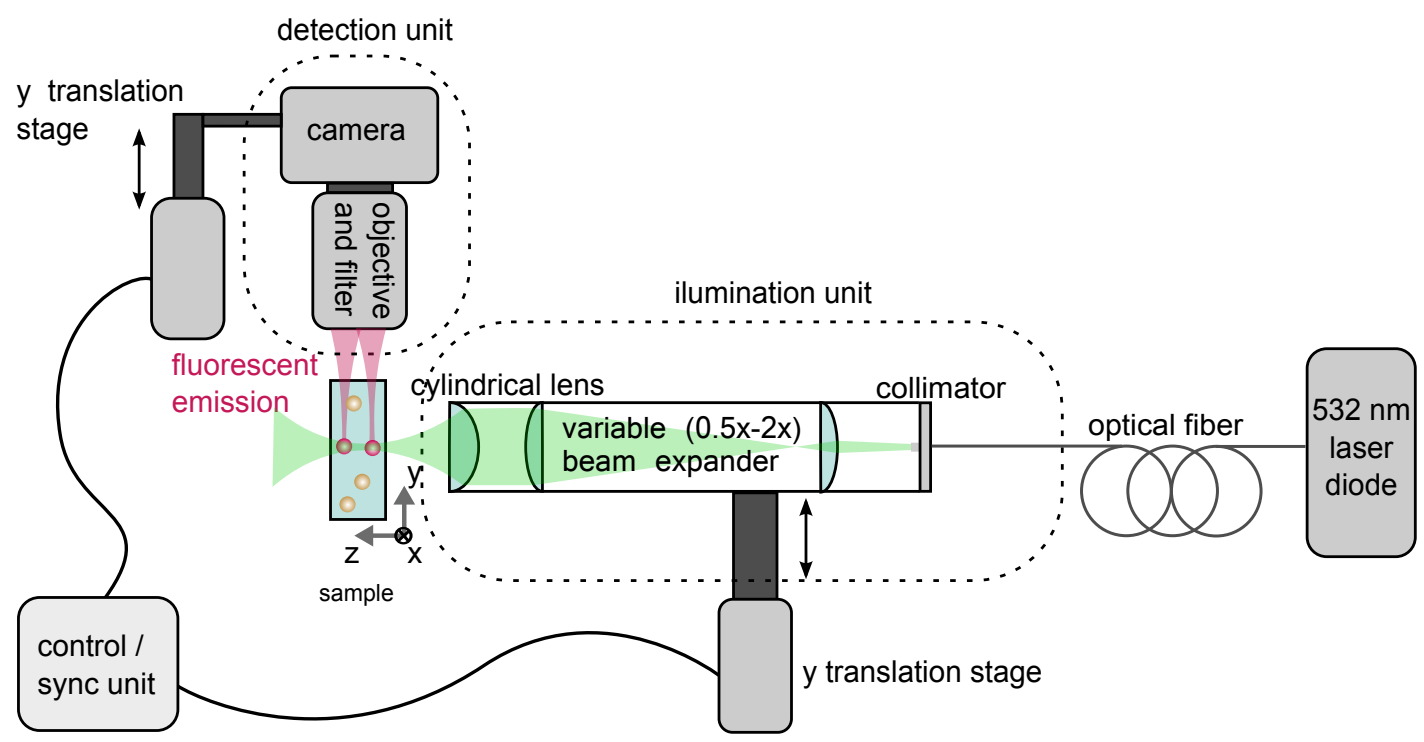

Fig. 4.9: Schematic illustration of the in-house built light sheet microscope. Generally, two parts are distinguished, the illumination and detection unit. Adapted from [139]. The definition of the $x, y$ and $z$-orientation is in accordance to the description in the main text.

As mentioned above, the key parts of the setup are the cylindrical lens and the capability of moving either the sample or the light sheet itself for scanning. In the setup shown, the illumination and detection unit are translated by stepper motors (8MVT70-13, Standa, Lithuania) and synchronized by a two-axis controller (8SMC4-USB-B9-2, Standa, Lithuania). It is possible to generate synchronized motion in a cyclic triangular profile with a step height of $5 \mu \mathrm{m}$ and an accuracy of $1 \mu \mathrm{m}$ at a maximum speed of $20 \mathrm{~mm} / \mathrm{s}$ and maximum travel range of $13 \mathrm{~mm}$. The stepper motors are controlled via a LabView program.

The illumination unit consists of a laser diode emitting light at $532 \mathrm{~nm}$ with a maximum output of $50 \mathrm{~mW}$ (DJ532-40, Thorlabs, USA). The laser diode is temperature and current controlled (TED200C \& LDC205C, Thorlabs, USA) for adjusting the output power. For achieving a nearly Gaussian beam with a $\operatorname{TEM}(0,0)$ profile and removing inherent divergence, the laser is coupled into a single mode fibre (P3-460B-FC-2, Thorlabs, USA) and collimated to a beam diameter of $2.1 \mathrm{~mm}$ (F220APC-532, Thorlabs, USA). Further change in beam diameter is realized by a $0.5 \times-$ $2 \times$ beam expander (BE052-A, Thorlabs, USA) before the light sheet is created by a cylindrical lens with a focal length of $50 \mathrm{~mm}$ and a diameter of $25.4 \mathrm{~mm}$.

The detection unit follows the basic principle of a brightfield microscope. It collects the fluorescent light perpendicular to the illumination by an infinity corrected $4 \times$ objective lens (UPlanFL N, NA $=0.13$, Olympus, Japan) and is focused by a $f=60 \mathrm{~mm}$ lens onto the near-infrared enhanced CCD chip of a high-speed camera (GS3-U3-41C6NIR-C, Point Grey, Canada). To prevent scattered laser light from reaching the CCD chip, a long pass filter with a cut-off below $600 \mathrm{~nm}$ (605 LP ET, AHF analysetechnik, Germany) is inserted into the beam 

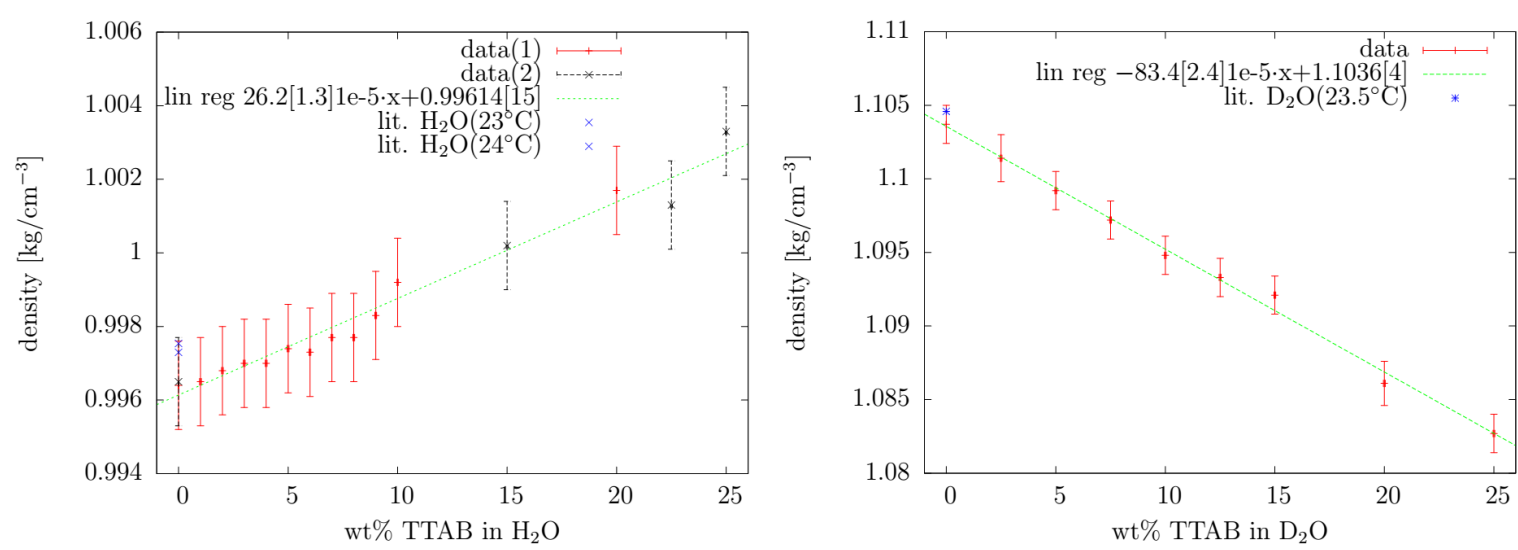

Fig. 4.10: Density of aqueous surfactant solutions for various surfactant concentrations $c_{s}$. Dissolved in $\mathrm{H}_{2} \mathrm{O}$ (left) and in $\mathrm{D}_{2} \mathrm{O}$ (right), measured with a pycnometer. Taken from [139].

path of the camera. Noteworthy, the infinity correction of the objective lens allows additional optical components to be inserted into the detection beam path.

Besides the adaption of LSFM, the second requirement for tracking active emulsions in three dimensions is the buoyancy matching of the continuous phase to generate droplet motion within a force free environment. In our case, this is achieved by admixture of heavy water to the surfactant solutions to obtain perfect density matching without altering the chemical properties of the solutions. A detailed description of the density matching is published in [139] and the calibration curves are shown in Fig. 4.10 for completeness. Knowing the density of 5CB and the concentration dependent densities of the $\mathrm{H}_{2} \mathrm{O}$ - and $\mathrm{D}_{2} \mathrm{O}$-TTAB solutions enables the calculation of the correct mixing ratios for the surfactant solutions at a given concentration.

Measuring the minimum sheet width is of utmost interest since this parameter defines the axial resolution and has strong implications on many of the experimental parameters of the light-sheet setup, as will be discussed below. For quantifying the sheet width, a commercial beam profiler (BP209-VIS, Thorlabs, USA) is used. This profiler makes it feasible to measure profiles in two perpendicular directions at the same time.

The measurement is executed in the rotating slit mode. The main idea of this mode is to measure narrow parts of the intensity distribution by rotating a narrow slit of width $w_{S}$ across the power meter [140]. Summation over the whole area of the aperture yields the intensity distribution along an orientation perpendicular to the optical path. For this method, the slit width has to be smaller than the beam to be measured. Following the assumptions of a Gaussian beam with a $\operatorname{TEM}(0,0)$ mode perpendicular to the propagation direction $(x, y$-plane), the intensity profile follows as [133]

$$
I=I_{0} \exp \left[-2\left(x^{2}+y^{2}\right) / w(z)\right]
$$


with the total intensity $I_{0}$, the coordinates $x, y$ and the beam width $w(z)$ following from eq. (4.6). The power $P$ of the laser results from integration of the intensity. Assuming that the measurement is executed along the $x$-direction and depending on the position $a$ of the slit, the integration boundaries are $\left(\left[a-w_{S} / 2, a+w_{S} / 2\right],[-\infty, \infty]\right)$ for $d x$ and $d y$, respectively. Assuming $w_{S} \rightarrow 0$, the measured power profile corresponds to the intensity distribution. By fitting a Gaussian function onto this profile, the $4 \sigma$-width as a measure for the sheet thickness is retrieved and plotted versus the distance $z$, as illustrated in Fig. 4.11. The minimum sheet thickness is $40 \mu \mathrm{m}$ in the focus, increasing to maximal $100 \mu \mathrm{m}$ at the edge of the field of view. Compared to the DOF resulting from eq. (4.5) and the objective properties $r_{\text {axial }} \approx 45 \mu \mathrm{m}$, the sheet width in the focus is more narrow and determines the axial resolution.

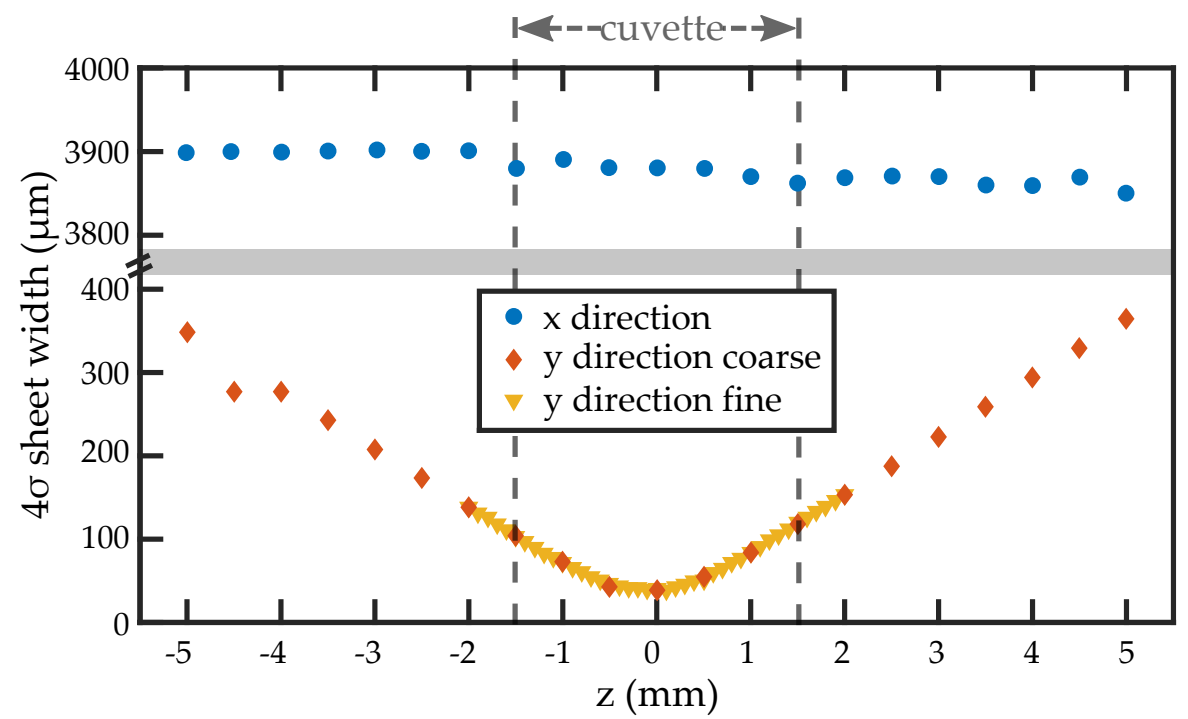

Fig. 4.11: $4 \sigma$ sheet width measured with the beam profiler along the $x$ and $y$ direction with $z$ being the propagation axis. The cuvette covers the $z$ axis from $-1.5 \mathrm{~mm}$ to $+1.5 \mathrm{~mm}$ (dashed lines). In $x$, a uniform width of $\approx 3.9 \mathrm{~mm}$ is measured, while the minimum width in $y$ is determined to $\approx 40 \mu \mathrm{m}$. The $y$-axis of the graph is increasing non-monotonously to cover the full extend.

Following the description of the setup and the determination of the sheet width, further measurement parameters have to be listed. The $x$-z plane is defined by the quartz cuvette (Hellma, 101.015-QS) to $3 \times 3 \mathrm{~mm}^{2}$ and the $y$-movement is fixed to $3 \mathrm{~mm}$. Calculating the confocal parameter in $z$-direction in accordance to eq. (4.7) gives $b=2 z_{R} \approx 4.7 \mathrm{~mm}$, exceeding the FOV.

The usual droplet diameter used in these measurements is $50 \mu \mathrm{m}$ and these droplets propel with an average speed of $5-25 \mu \mathrm{m} / \mathrm{s}$. Hence, droplets roughly move one radius per second. The size of the droplet further determines the inter-frame $y$-displacement. Here, it is desirable to record on average 2 - 3 slices per droplet. Therefore, an inter-frame movement in $y$ should not exceed $30 \mu \mathrm{m}$. This is determined by the ratio of the frame rate and the speed of the stepper motor. The camera is capable of recording images at $90 \mathrm{fps}$ for the full CCD-chip size of 
$2048 \times 2048 \mathrm{px}^{2}$. Taking the magnification of the objective and the cross section of the cuvette into account, it is possible to decrease the field of view to $928 \times 928 \mathrm{px}^{2}$. These recording settings result in droplet projections of $15 \mathrm{px}$ in diameter on the CCD chip, which is sufficient for droplet tracking. Considering the maximum data transfer rate for USB 3 of $130 \mathrm{Mbyte} / \mathrm{s}$, frame rates up to $150 \mathrm{fps}$ can be realized. With the requirement for the inter-frame movement the speed of the stepper motors is limited to $4.5 \mathrm{~mm} / \mathrm{s}$, resulting in a temporal duration of a full cycle, one upward and one downward movement, of $1.33 \mathrm{~s}$ which corresponds approximately to 0.75 cycles $/ \mathrm{s}$. Furthermore, this guarantees a maximum displacement of droplets of one diameter per cycle.

One further restriction needs to be mentioned at this point. While the light absorption of 5CB at $532 \mathrm{~nm}$ is negligible, the refractive index mismatch between aqueous phase and droplet phase is on average $\Delta n \approx 0.3$, resulting in strong scattering of both, excitation and fluorescence [91]. Due to this mismatch and for fluorescence signal to reach the detector, the droplet number density has to be in the range of the single scattering limit. With this assumption a volume fraction of $\approx 1.5 \times 10^{-3}$ can be derived following the anomalous diffraction approximation [141]. This volume fraction results in a total number of approximately 2000 droplets for the given volume of the cuvette, setting an upper limit to the capability of droplet tracking. In general, refractive index matching of the LC and the aqueous phase is not feasible, due to the optical anisotropy of the LC.

After taking care of the experimental parameters, the output of LSFM experiments are three data files. The first file is the video recorded by the CCD camera, the second and third are text files with temporally resolved frame positions and the stepper motor positions, respectively.

\subsection{Particle tracking}

In active matter, tracking single particles in acquired recordings and subsequently identifying their individual trajectories is the foundation for further data analysis. Thus, the following section will be separated into two parts: image processing and successive trajectory reconstruction.

\subsubsection{Image processing}

The single images from an experimental data set are used for determining the $x-z$ positions of all droplets in the field of view. For this, a blob detection algorithm is used, based on thresholding of single frames to generate binary images. The centres of mass of the particles are determined by topological structural analysis of contiguous white areas [142], assuming 
spherical structures and fitting disks to the contours in the image. Deviations from spherical contours are used for further analysis, comparing the area of the fitted disk to the enclosed area by the contour. For high deviations from circular contours, an analysis for convexity defects follows in order to separate close particles. The result is the centre of mass position of a single centroid as well as the area of the fitted disk [143].

For data recorded in LSFM, an additional step is necessary before the same processing as in 2D trajectory reconstruction is applied. Here, it is important to locate the $x y z$ centres of mass of centroids, which result from a number of slices recorded along an individual droplet in a single scanning cycle. First, the $y$-coordinates for respective centroids are obtained by combining the temporal information of the recorded video and the movement profile of the translation stages. Second, individual droplets are identified from spatial clusters of $x y z$ coordinates in a single scanning cycle using the mean shift method [144]. Finally, this results in temporally resolved $x y z t$ coordinates of determined droplets.

\subsubsection{Trajectory reconstruction}

After obtaining the coordinates of single centroids, individual particle trajectories are reconstructed, using a simple Crocker-Grier type nearest-neighbour approach [143].

Starting with the coordinates from the first frame or 3D image stack, respectively, the nearest neighbours within a given area estimated by the average droplet speed are determined in the consecutive unit. If no candidate has been found, the preceding trajectory is used to calculate a displacement vector, which is used to predict the direction and distance of the associated droplet. If this prediction fails for a given number of successive frames, usually two to three, the droplet is deemed lost and the trajectory is closed. This finally results in connected timeresolved $x z t$ trajectories in $2 \mathrm{D}$ and $x y z t$ trajectories in $3 \mathrm{D}$ for each identified droplet.

In the specific case of tracking in this thesis, we have developed a Python code using the OpenCV library API for image processing, with a graphical user interface to conveniently set and check parameters like binarization thresholds or particle sizes.

\subsection{Determination of flow profiles}

In microfluidic flows or in active systems, knowledge of the generated flow field and local velocities is highly desirable. In recent years, the technique of Particle Image Velocimetry (PIV) has been used more and more often [145]. The basic idea is to seed the liquid with a high number of small fluorescent tracer particles, usually with sizes in the range of $100-300 \mathrm{~nm}$ [146]. By recording the displacement $\delta \mathbf{r}$ of these particles at short time intervals $\delta t(\approx 100 \mathrm{~ms})$, the 
velocity $u=\delta \mathbf{r} / \delta t$ of the tracer particles can be obtained. Besides the small size, the particles should have a density close to that of the fluid for two reasons: first, to follow the streamlines accurately without altering the hydrodynamics and second, to not sediment to the ground [22, 146]. A light sheet or high magnification objective lenses with small DOF are used for narrowing the illuminated volume [129]. The displacement vectors are extracted by using an auto-correlation approach on pairs of images. In more detail, not the displacement of a single particle is considered, but rather correlations between interrogation areas in consecutive images. This results in velocity vectors distributed on a uniform grid with a resolution determined by the minimum interrogation area. For only one pair of images, the result is noisy and high fluctuations are visible but by averaging over many of such pairs, a smooth velocity profile is obtained [146]. The auto-correlation is given by [22]

$$
C(\delta \mathbf{r})=\sum_{i, j} I_{1}\left(x_{i}, y_{i}\right) I_{2}\left(x_{i}+\delta x, y_{i}+\delta y\right)
$$

with the intensity $I_{k}$ of the $k$-th image, the coordinates $x_{i}, y_{i}$ of the pixel and the components $\delta x, \delta y$ of displacement $\delta \mathbf{r}$. The value of $\delta \mathbf{r}$ with the highest $C(\delta \mathbf{r})$ corresponds to the displacement of the fluid in this interrogation area. Taking the time difference $\delta t$ into account, a velocity can be attributed to the area.

A second method that is extensively used for tracking the flow profile is applied if the number of seed particles is low and therefore bases on single tracer detection. The so-called Particle Tracking Velocimetry (PTV) [147] determines inter-frame displacements for single particles and after sectioning the whole field of view into interrogation areas, the velocities are averaged and interpolated onto a uniform grid to obtain the flow profile [148].

In most of our cases, a high number of tracer particles is used. Instead of using fluorescent particles, an alteration of the technique is employed, called Particle Shadow Velocimetry (PSV) [149]. Here, the scattering and absorption of the seed particles is recorded in transmission microscopy. This results in an inverse PIV signal, implying dark spots on a bright background. In our case, this has the advantage that the swimming, non-fluorescent droplet can be observed simultaneously. The basic analysis procedure is described in Fig. 4.12 .

Usually, a measurement consists of a video of a droplet transition at high magnification, typically at least $60 \times$ at $N A>0.7$, and at high frame rates of $50-100 \mathrm{fps}$. To show that the high magnification objective lens delivers a sufficiently narrow DOF, the measurement depth $\delta z_{m}$ is calculated by following the reasoning of Meinhart et al. [129]. Thus,

$$
\delta z_{m}=\frac{3 n \lambda_{0}}{N A^{2}}+\frac{2.16 d_{p}}{\tan \theta}+d_{p}
$$


with the refractive index $n$ of the medium between objective and sample, the particle diameter $d_{p}$, the numerical aperture $N A$, the collection angle of the objective $\theta=\sin ^{-1}\left(\frac{N A}{n}\right)$ and the wavelength $\lambda_{0}$ of the monochromatic light source [129]. To give a specific example of $\delta z_{m}$, values for a $60 \times$ air objective (LUCPLFL, Olympus, Japan), $\lambda_{0}=530 \mathrm{~nm}$ and $d_{p}=1 \mu \mathrm{m}$ result in $\delta z_{m} \approx 6.4 \mu \mathrm{m}$. When considering a droplet diameter of $d>60 \mu \mathrm{m}, \delta z_{m}$ is close to $10 \%$ of the diameter and thus, a reasonable measurement depth for the PSV analysis.

After the video recording, every image is, in a first step, thresholded and binarized. The second step inverts and centres the frame onto the droplet centre to change into the droplets' frame of reference. This delivers the final image sequence for analysis with PIVLab, an open source software based on MatLab (Mathworks, USA) [150, 151]. Averaging over at least 40 image pairs results in the final velocity profile allowing extraction of data like the velocity magnitude and the tangential components of the velocity around the droplet swimmers.

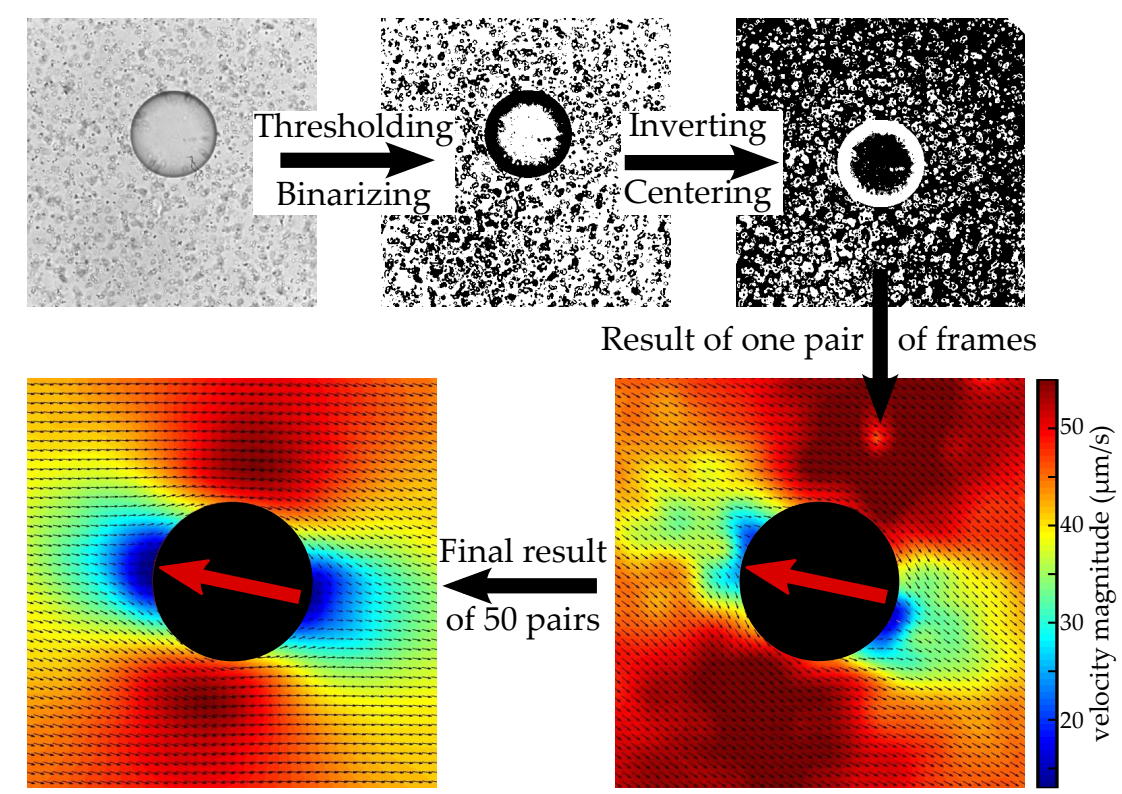

Fig. 4.12: Example for PSV analysis following the described procedure. The processed images in the bottom row are plotted with the same colour code and boundaries. Red arrows emphasize the propulsion direction. 



\section{Results}

This chapter is devoted to the outcome of this thesis. The results will be presented in a fashion of increasing system complexity. The elucidation of the one-dimensional propulsion realized within a capillary is followed by the depiction of two-dimensional measurements of single droplets and large ensembles of swimmers. Finally, three-dimensional experiments will be discussed, covering the range from single swimmer dynamics to the interaction of swimmers in a dense suspension.

\subsection{Droplet motion in one dimension}

\subsubsection{Solubilization experiments}

In the theoretical part of this thesis, the two different solubilization mechanisms of oil droplets in surfactant solutions have been mentioned. It has also been outlined that only the molecular pathway will result in a self-sustained Marangoni flow due to depletion and advection (see Sec. 2.3). For the proof of this concept and to quantify the solubilization rates in different surfactant concentrations, we performed long time experiments of solubilizing droplets. For this experiment, a small number of droplets with sizes between $50-120 \mu \mathrm{m}$ in diameter are inserted into a reservoir that is filled with the surfactant solution of the desired concentration. We assured that the total volume fraction of $5 \mathrm{CB}$ inside the surfactant solutions stays reasonably low to prevent the solution from being saturated. Brightfield images are taken every two to five minutes and the sizes of the droplets are retrieved from the images. The results are shown in Fig. 5.11, depicting the change in droplet size $u=d R / d t$ vs. the surfactant concentration $c_{s}$. The linear relation between $u$ and $c_{s}$ confirms previous investigations [65]. However, in the present study, a larger range of concentrations as compared to previous works is tested. Qualitatively, the solubilization rate increases after the CMC $\left(c_{C M C}=3.87 \mathrm{mM}\right.$ $=0.13 \mathrm{wt} \%$ ) is reached and shows an asymptotic behaviour for surfactant concentrations from the $\mathrm{CMC}$ to $3 \mathrm{wt} \%$. Then, the rate increases linearly with the surfactant concentration up to the upper limit of tested concentrations. The transition between the asymptotic regime and the range of linear increase coincides roughly with the movement threshold for large droplets, as will be shown in Sec. 5.2.3

An analysis scheme for the solubilization in surfactant solutions, called the Todorov model, is found in literature [152]. We applied this scheme to the asymptotic regime, after the CMC is exceeded. Basically, this analysis takes into account that no filled micelles are present inside a diffusion layer of extent $\kappa^{-1}$ around the droplet, indicating that micellar diffusion is faster than micellar solubilization. As mentioned in Sec. 2.3 , this boundary layer is small compared to the 


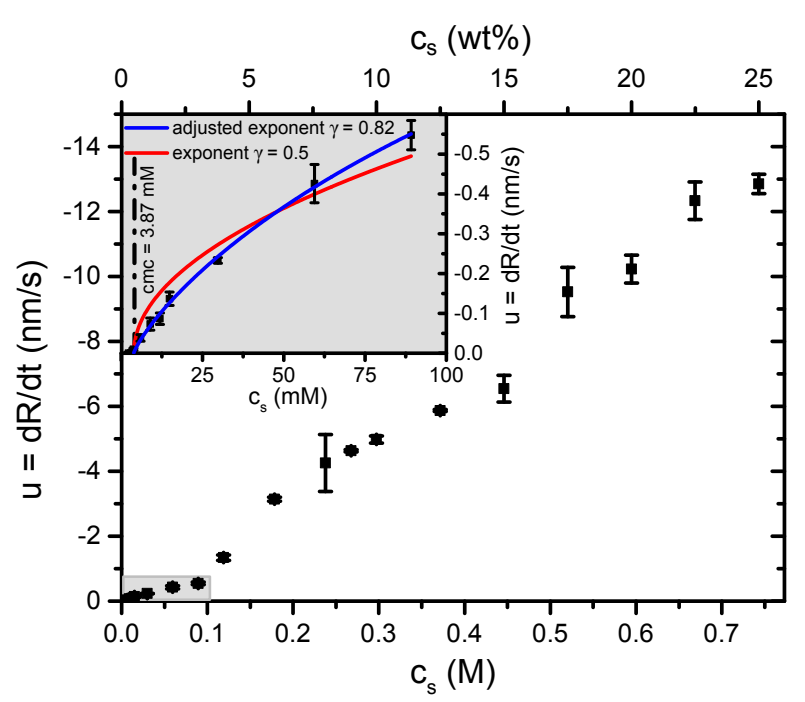

(a)

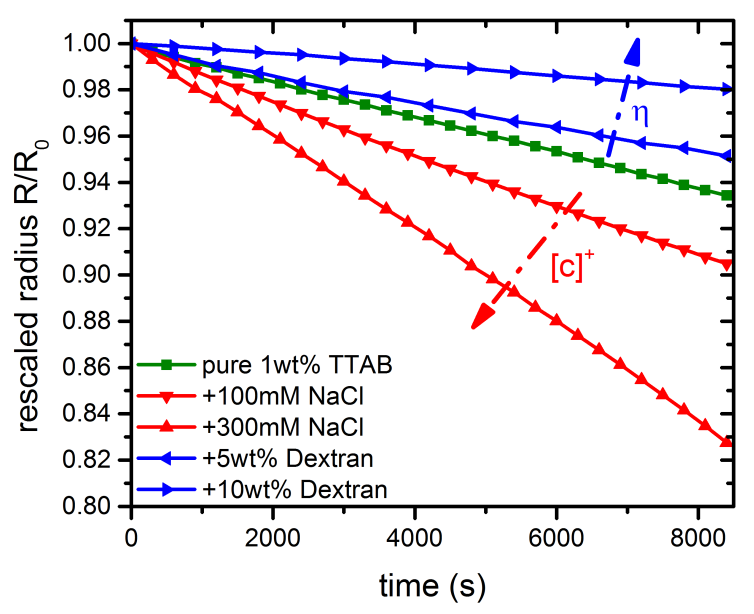

(b)

Fig. 5.1: Solubilization experiments of $5 \mathrm{CB}$ in TTAB solutions. (a) Solubilization rate $u$ vs. surfactant concentration $c_{s}$. The inset emphasizes the regime analysed by the Todorov model $(0 \mathrm{mM}$ to $100 \mathrm{mM})$. For large surfactant concentrations, a linear increase of $u$ evolves. Scale in $w t \%$ is given atop. (b) Decrease of droplet diameters over time in $c_{s}=1 \mathrm{wt} \%$ TTAB solutions, pure (green) and with additives (red and blue). An increase in viscosity $\eta$ is achieved by adding Dextran to the continuous phase (blue graphs). $\mathrm{NaCl}$ addition increases the ion amount $[c]^{+}$, thus altering the electrostatic repulsion (red graphs). The slopes of the lines reflect the solubilization rates in the varying conditions.

droplet's radius. Taking only active, i.e. not yet filled, micelles into account and calculating the local concentration of oil molecules with the appropriate boundary conditions yields the final equation for the solubilization rate $u$ [152]. In particular,

$$
u=\frac{d R}{d t}=\frac{a\left(c_{s}-\mathrm{CMC}\right)^{\gamma}}{b+\left(c_{s}-\mathrm{CMC}\right)^{\gamma}}=\frac{a c_{s 0}^{\gamma}}{b+c_{s 0}^{\gamma}}
$$

with system parameters $a$ and $b$ and $\gamma=0.5$ for the pure Todorov model. For 5CB droplets in TTAB solutions, a slightly differing exponent $\gamma$ is needed to fit the model satisfactorily and to obtain reasonable values for $a$ and $b$. Instead of $\gamma=0.5$ (red fit in Fig. 5.17), a change to $\gamma=0.82$ (blue fit) offers better results. Since the Todorov model only considers resting droplets in surfactant solutions, this calculation is not able to reproduce the linear regime observed for high surfactant concentrations in the above experiments. The slight change of the exponent might result from an additional mass flux into the LC droplet [153], which is neglected in the Todorov model. A further reason for the required adaption is that the dynamics of micelles are influenced by the surrounding liquid and interface. If a micelle is filled, additional single molecules are needed to accommodate the micelle's expansion. This results in a local depletion below the CMC. If this depletion gets large enough, empty micelles disso- 
ciate to replenish the $\mathrm{CMC}$ and thus, the number of active micelles is decreasing. Moreover, Todorov et al. considered the surfactant SDS which might react differently than TTAB.

However, using the fit-parameters $a$ and $b$ and the solubilization scheme from Todorov et al. provides two important factors, the mass-transfer coefficient $\alpha$ and the rate constant of solubilization $k_{+}$. The scheme is similar to calculation procedures for $n$-Decane mentioned in Ref. [152]. The two relations are given by

$$
\begin{aligned}
& \alpha=\frac{a}{\beta}=\frac{a}{v_{o i l} D_{o i l} c_{e q}}, \\
& k_{+}=D_{o i l} N_{a g g}\left(\frac{\alpha}{b}\right)^{2}
\end{aligned}
$$

with $\beta$ purely depending on the oil properties, the total volume $v_{\text {oil }}$ and the diffusivity $D_{\text {oil }}$ of single oil molecules, the equilibrium concentration $c_{e q}$ of oil in water and $N_{a g g}$ as the aggregation number for micelles. $N_{a g g}$ is set to 100 for the following approximations (see Sec. 3.1. The knowledge of $k_{+}$allows us to calculate the extent of the solubilization zone $\kappa^{-1}$ via

$$
\mathcal{\kappa}^{-1}=\frac{1}{\sqrt{\frac{k_{+}\left(c_{s}-\mathrm{CMC}\right)}{N_{a g g} D_{\text {oil }}}}} .
$$

The parameters and calculated values are summarized in Tab. 5.1 .

Here, we approximated the diffusivity of the oil molecules via the Perrin equation [154] for prolate ellipsoids with a main axis of $d=20 \AA$. We have no tabulated values for the equilibrium solubility $c_{e q}$ of $5 \mathrm{CB}$ in water, but according to UV-absorbance measurements reported on in Ref. [155], it should be on the order of $\mu \mathrm{M}$. For both values of $\kappa^{-1}$, obtained for different surfactant concentrations, the zone of solubilization is two orders of magnitude smaller than the droplet radius of at least $R_{0}=25 \mu \mathrm{m}$. Hence, the approximation of a narrow solubilization area around the droplet (see Sec. 2.3) is appropriate.

A qualitative determination of the pathway of solubilization was conducted with additives to the surfactant solution. We assume the prevalence of the molecular pathway from the ionic nature of TTAB. We verified this assumption by adding sodium chloride or dextran to the surfactant solutions, thereby modifying screening length and viscosity, respectively. All experiments were executed with a constant surfactant concentration $c_{s}$ of $1 \mathrm{wt} \%$ and thus, the droplet is stationary. Figure $5.1 \mathrm{p}$ shows the time-dependent diameter change for the varying conditions. The green graph displays the behaviour for a pure TTAB solution and serves for comparison of the solubilization rate. $\mathrm{NaCl}$ was used to alter the screening of the droplet surface charge by ions and dextran was used to increase the viscosity of the surfactant solution. 
Tab. 5.1: Parameters used in and obtained from the calculation of the Todorov model.

\begin{tabular}{cc}
\hline Parameter & 5 CB \\
Mass Density $\left(\mathrm{kg} / \mathrm{m}^{3}\right)$ & 1022 \\
$v_{\text {oil }}\left(\mathrm{m}^{3}\right)$ & $4.052 \times 10^{-28}$ \\
$D_{\text {oil }}\left(\mathrm{m}^{2} / \mathrm{s}\right)$ & $4.83 \times 10^{-10}$ \\
$c_{e q}\left(\mathrm{~m}^{-3}\right)$ & $10^{19}$ \\
$\beta\left(\mathrm{m}^{2} / \mathrm{s}\right)$ & $1.17 \times 10^{-16}$ \\
\hline Values from fit & 2.27 \\
$a\left(\mathrm{~nm}^{2} \mathrm{~s}\right)$ & 0.41 \\
$b\left(\mathrm{M}^{1 / 2}\right)$ & 19.3 \\
\hline calculated values & $1.85 \times 10^{-19}$ \\
$\alpha\left(\mu \mathrm{m}^{-1}\right)$ & 0.45 \\
$k_{+}\left(\mathrm{m}^{3} / \mathrm{s}\right)$ & 0.071 \\
$\kappa^{-1}(\mu \mathrm{m})$ for $0.2 \mathrm{wt} \%$ & \\
$\kappa^{-1}(\mu \mathrm{m})$ for $3 \mathrm{wt} \%$ & \\
\hline
\end{tabular}

Addition of sodium chloride at concentrations of $100 \mathrm{mM}$ and $300 \mathrm{mM}$ (Fig. 5.1p, red lines) increases the ion amount inside the continuous phase. Thus, the charges on the droplet surface are strongly screened. This results in micelles approaching closer to the droplet surface, while attaching is still suppressed. Due to the decreased diffusion layer ( $\Lambda$ in Fig. 2.11), the LC molecules are incorporated closer to the droplet surface and the droplets shrink faster. However, we have to consider the influence of the salt concentration on the micelles. In Ref. [156] an increase in micelle size with salt concentration is reported on and this will furthermore influence the total solubilization rate.

Dextran $\left(\mathrm{C}_{6} \mathrm{H}_{10} \mathrm{O}_{5}\right)$ has a molecular weight $M_{W}$ of $70 \mathrm{kDa}$ and was used at weight fractions of $5 \mathrm{wt} \%$ and $10 \mathrm{wt} \%$. The viscosity of the bulk liquid increases significantly $(\approx 3 \times$ at $5 \mathrm{wt} \%$ [157]) with addition of dextran. We confirmed that the solubilization rate decreases (see Fig. 5.1p, slopes of blue graphs), since the diffusion constants of the partaking species are lowered. It was reported that in the micellar pathway, the change of the diffusion constant of micelles does not influence the solubilization rates strongly [158]. The latter scheme is interfacially dominated, since the attaching, filling and detaching of micelles from the droplet surface defines the reaction rate. The molecular pathway, however, is clearly diffusion dominated. Thus, the decreased solubilization rate with viscosity increase is a strong indication to the molecular pathway. 
To summarize, the experiments with additives strongly suggest that the molecular pathway is favoured over the micellar pathway. This verification is in accordance with the theoretical assumptions (see Sec. 2.3.

\subsubsection{D movement in capillaries}

The first observations of moving LC droplets were reported on in Refs. [65, 98], including measurements of droplet velocities in square glass capillaries. This is the starting point for the investigation of active emulsions and thus, the first step in the current thesis was to confirm the droplet propulsion behaviour of Refs. [65, 98]. Initially, we produced droplets of $50 \mu \mathrm{m}$ in diameter with a microfluidic flow-focusing device (see Sec. 4.2) and square glass capillaries with inner cross sections of $50 \mu \mathrm{m}^{2}$ (CM Scientific Ltd., UK) served as observation chambers. After mixing the surfactant solution with a small number of $5 \mathrm{CB}$ droplets, filling the capillary by means of capillary forces and sealing the capillary, the propulsion of droplets was recorded. Image-processing provided the $x y$ positions of the droplets and the velocity of the droplet was calculated from the inter-frame displacement and the frame rate. Fig. 5.2 1 depicts the mean velocity vs. the surfactant concentration $c_{s}$ over the entire concentration range of $6-25 \mathrm{wt} \%$. The qualitative behaviour of the droplet velocity is in good agreement with the description of Sec. 2.3. Here, the propulsion in dependence of the driving parameter $\beta$ was analysed (see Fig. 2.11k). This behaviour can be translated to our results. A distinct onset of droplet propulsion at a critical driving parameter $\beta_{c}$, specifically at $c_{s}=6 \mathrm{wt} \%$, is followed by an

(a)

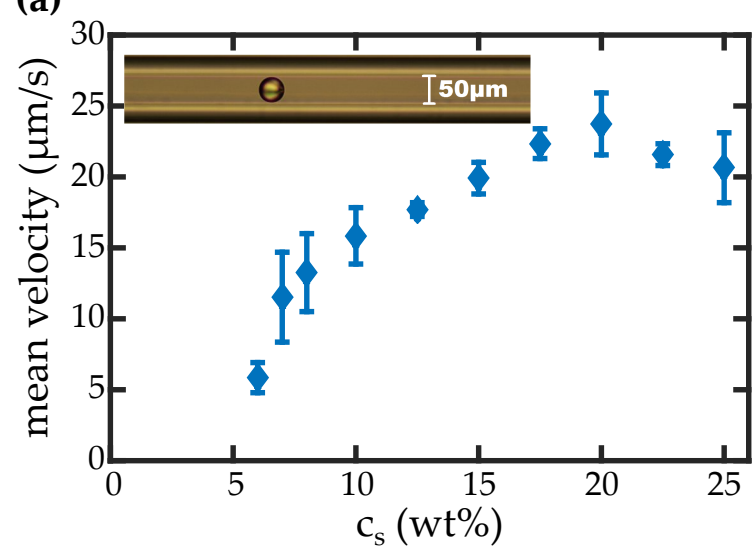

(b)

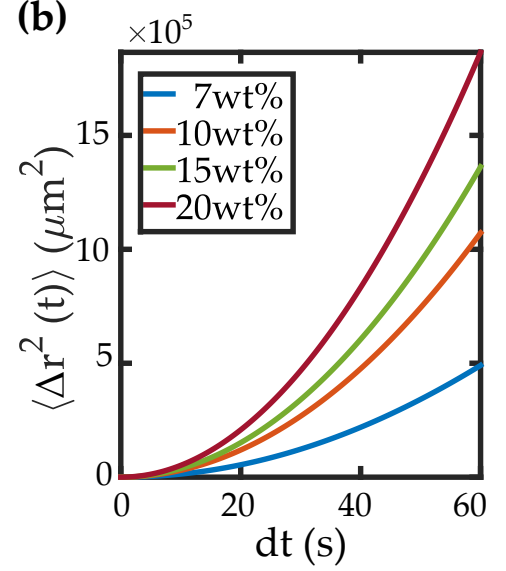

Fig. 5.2: (a) $1 \mathrm{D}$ velocity of $50 \mu \mathrm{m}$ sized droplets in a capillary. The graph shows the behaviour of the mean velocity vs. the surfactant concentration $c_{s}$. Distinct start of propulsion, increase in velocity and a final decrease at high concentrations can be observed. The inset shows an experimental micrograph with one polarizer oriented along the capillary axis. Figure adapted from [4]. (b) Mean squared displacement calculated for one-dimensional experiments with $c_{s}=7,10,15$ and $20 \mathrm{wt} \%$. The MSD is depicted in linear scale over a period of $60 \mathrm{~s}$. The general behaviour of the data follows a $v^{2} t^{2}$ dependence as expected for ballistic motion. 
increase of the mean velocity up to $23 \mu \mathrm{m} / \mathrm{s}$ at $c_{s}=20 \mathrm{wt} \%$. The decrease in velocity for high surfactant concentrations results from increasing rotational diffusion and thus, the droplet is arrested at the capillary boundaries before it reorients back along the long axis of the capillary.

For the same data set, the mean squared displacement was calculated via eq. 2.19). To analyse the different temporal behaviour of the swimmers, the limiting cases for small and large $t$ as compared to the persistence time $\tau_{r}$ (see eq. (2.24)) were considered following the description of Howse et al. [55]. In the case of propulsion in a capillary, the diffusive regime will not be reached even for long time scales. This holds true for droplet diameters that are comparable to the cross section of the capillary, since the droplets are guided by the walls. The results for $c_{s}=7,10,15,20 \mathrm{wt} \%$ for an elapsed time of $60 \mathrm{~s}$ are shown in Fig. 5.2 $\mathrm{p}$. The difference in speed is clearly visible from the respective slopes of the mean squared displacements, following the $v^{2} t^{2}$-behaviour.

(a)

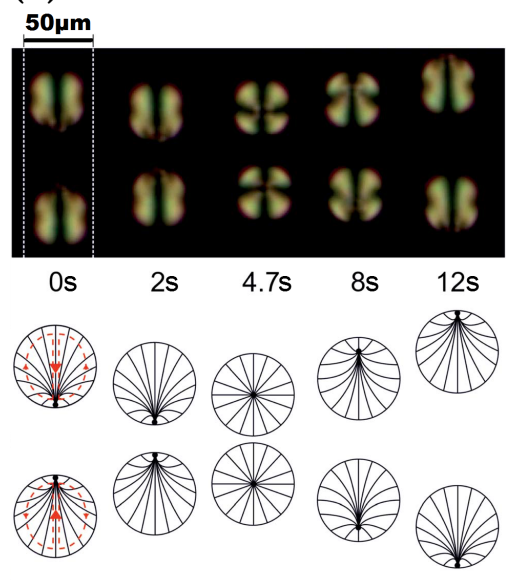

(b)

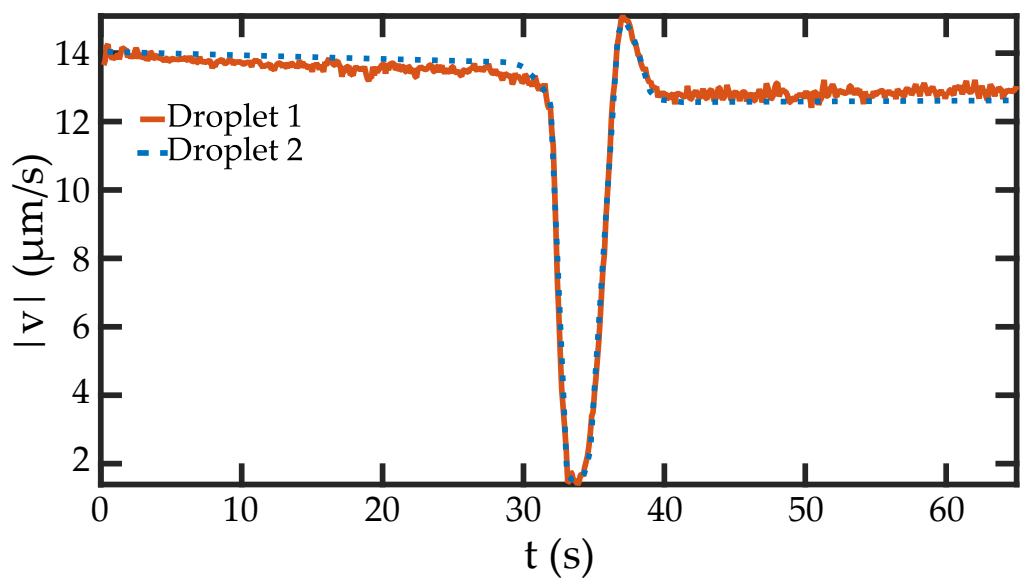

Fig. 5.3: (a) (top) Micrographs of collision event in 1D over the course of $12 \mathrm{~s}$. The dotted line in the left picture indicates the dimensions of the capillary, not visible under crossed polarizers. (bottom) Depiction of director structure of the droplets for the different situations. The black spot marks the defect position and the red dashed line in the left column emphasizes the internal convection with the red arrows pointing into the flow direction. Taken from [4]. (b) Speed $|v|$ vs. time $t$ for an exemplary collision event in a capillary. The mean droplet speed before and after collision is $|v| \approx 14 \mu \mathrm{m} / \mathrm{s}$. The full collision event takes approximately $6 \mathrm{~s}$ and the total recording has a duration of $65 \mathrm{~s}$.

We continue the investigation by focusing on droplet collision dynamics of two droplets in a capillary (see Fig. 5.3). If two droplets collide in the capillary, the droplets touch and reorient into opposite directions, accelerate and reach the mean velocity again shortly after reorientation. Using polarized microscopy, the orientation of the droplets can be visualized (Fig. 5.3.). The whole collision event has a duration of $\approx 12 \mathrm{~s}$ and the reorientation after the collision is clearly observed from the internal droplet structures. Below the micrographs, a schematic illustration of the director profile during the event is shown, with the black spot indicating the defect position and the red dashed line in the left column emphasizing the internal convection. 


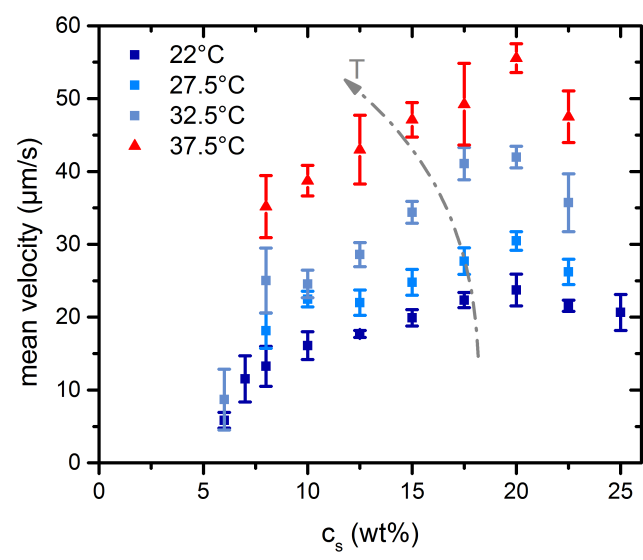

(a)

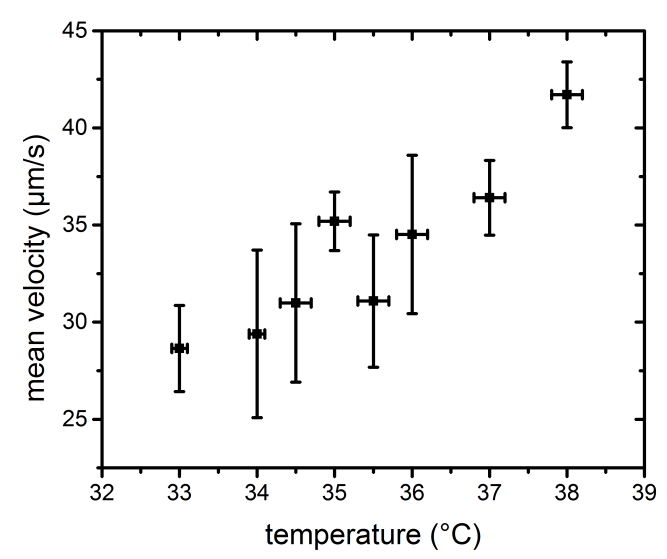

(b)

Fig. 5.4: Temperature dependent propulsion in 1D. (a) Mean velocity vs. surfactant concentration $c_{s}$ for temperatures of $22,27.5,32.5$ and $37.5^{\circ} \mathrm{C}$. The last setting exceeds the temperature for the nematicto-isotropic transition. The increase in temperature results in an overall increase of the velocity, while the transition to the isotropic phase does not inhibit propulsion. (b) Mean velocities around the nematic-to-isotropic transition. A small decrease in velocity is observed close to the transition point of $T_{N I}=35.5^{\circ} \mathrm{C}$.

In Fig. $5.3 \mathrm{~b}$, a more detailed observation of a collision event in terms of the local velocities is shown. In this example, two droplets approach each other and collide in the centre of the field-of-view. The whole recording takes $65 \mathrm{~s}$, while the collision and reorientation takes roughly $6 \mathrm{~s}$. The mean velocity of the droplets before and after collision is $14 \mu \mathrm{m} / \mathrm{s}$. The collision abruptly decelerates the droplets and afterwards, the droplets accelerate over a short duration. Here, they even surpass the mean velocity until they reach the mean velocity again due to the force balance of viscous drag and propulsion. The increase in speed shortly after the collision is due to a repulsion resulting from negative autochemotaxis (see Sec. 5.1.4).

So far, the surfactant concentration was considered as the driving parameter of propulsion. In the following, the effect of temperature and of the liquid crystalline phase will be investigated. The surfactant concentration range is kept between 6 and $25 \mathrm{wt} \%$ TTAB as long as feasible. However, for high temperatures and high surfactant concentrations, the solubilization is very fast, such that recording the droplets at an appropriate initial diameter is impossible. This sets the upper limit of surfactant concentrations tested. The temperature of the capillaries was controlled by a heating stage attached to a thermometer.

The results are shown in Fig. 5.4 for temperatures varying from 22 to $37.5^{\circ} \mathrm{C}$ (see colour code in Fig. 5.4a). Square symbols in shades of blue indicate the nematic phase, while red triangles show the velocities of droplets above the nematic-to-isotropic transition. An overall increase in velocity is observed for an increasing temperature, while propulsion is still maintained when the liquid crystalline phase changes. This indicates the independence of the propulsion 
mechanism on the nematic character (see also Ref. [65]). The increase in velocity is easily understood by an acceleration of diffusion dynamics, solubilization rate and decrease of bulk fluid viscosity. More intriguing, however, is the behaviour close to the nematic-to-isotropic transition (see Fig. 5.4 $\mathrm{p}$ ), with the transition temperature of $T_{N I}=35.5^{\circ} \mathrm{C}$. Here, a small decrease in speed at the transition is observed, suggesting a minor effect either of the nematic ordering or of the transition dynamics on the droplet propulsion speed. After the small dip, the approximately linear increase in velocity with temperature is again observed.

\subsubsection{Estimation of Marangoni strength}

For an estimate of the generated interfacial-tension gradients $\nabla \gamma$ that result in Marangoni flows, the calculations mentioned in Sec. 2.3 are applied to the experiments and compared to the equilibrium interfacial tension of 5 CB droplets in TTAB solutions. Recalling Sec. 2.3, the basic equation for this relation results from the comparison of viscous drag force times the velocity and the energy dissipated due to friction. Eqs. 2.5) and 2.6) can be rewritten to

$$
\begin{aligned}
F_{\text {visc }} V & =E_{\text {diss }} \\
2 \pi R \eta_{c} V^{2} \frac{2+3 \mu}{1+\mu} & =4 \pi R^{2} V \nabla \gamma \\
\nabla \gamma & =\eta_{c} \frac{V}{R} \frac{2+3 \mu}{2(1+\mu)}
\end{aligned}
$$

with the viscosity of the continuous phase $\eta_{c}, R$ and $V$ as the droplet's radius and velocity and $\mu$, the ratio of $\eta_{d}$ and $\eta_{c}$. In this case, we chose $\eta_{d}=\bar{\eta}_{1,2,3}=0.053 \mathrm{~Pa} \cdot \mathrm{s}$ and $\eta_{c}$ depending on the surfactant concentration $c_{s}$ between 0.0123 and $0.0204 \mathrm{~Pa} \cdot \mathrm{s}$ (estimated from Ref. [98]), $R=25 \mu \mathrm{m}$ and the velocities in accordance to Fig. 5.2.

The blue squares in Fig. 5.5 show the data for $\nabla \gamma$, which range from 0.3 to $2.5 \mathrm{mN} / \mathrm{m}^{2}$. The total difference in interfacial tension $\Delta \gamma$ along the droplet surface follows now from the gradient $\nabla \gamma$ and the respective length of the surface $\delta x(\Delta \gamma=\nabla \gamma \cdot \delta x)$. Since we are interested in the difference between the leading and the rear end we consider the circumference of one hemisphere of a droplet with $\pi R$ as the length scale of interest. For droplets with a radius of $25 \mu \mathrm{m}$, an interfacial tension difference $\Delta \gamma$ of approximately $10^{-5}$ to $10^{-4} \mathrm{mN} / \mathrm{m}$ is derived.

To compare now the obtained values of $\Delta \gamma$ with the equilibrium interfacial tension $\gamma$, pendant drop measurements of millilitre sized 5CB droplets for varying surfactant concentrations were performed. In such a configuration, the interfacial tension is derived from the shape change of the droplets circumference under gravity. Up to a surfactant concentration $c_{s}$ of $12.5 \mathrm{wt} \%$ $\mathrm{TTAB}$, this measurement is feasible. 


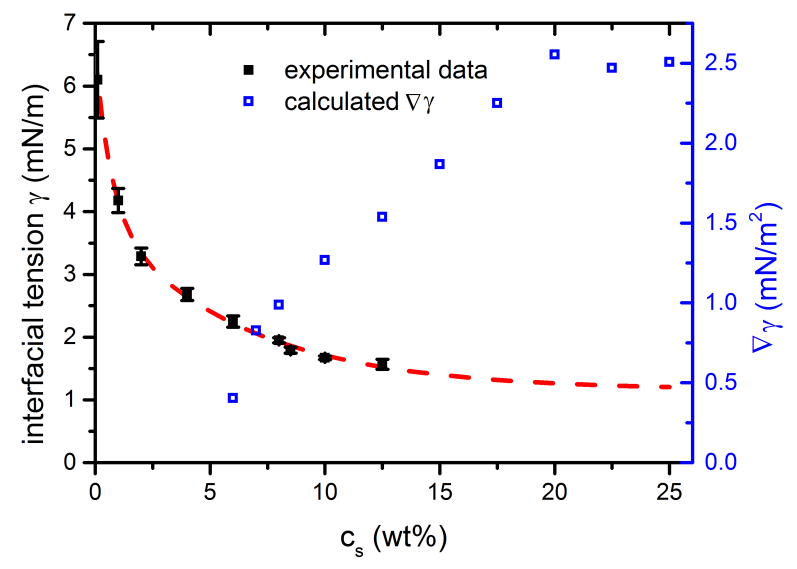

Fig. 5.5: To estimate the interfacial-tension gradients $\nabla \gamma$, the 1D velocity and the bulk viscosity are considered, following eq. (5.5) (blue squares). For a better understanding, the calculated values are compared to the equilibrium interfacial tension measured in a pendant drop assembly (black squares). The red dashed line is a guide to the eye for higher surfactant concentrations.

For higher concentrations, it is extrapolated by an exponential fit (red dashed line in Fig. 5.5), since a stable droplet is not guaranteed any more due to strong surface flows. The latter will compress the surfactant molecules on the interface beyond the equilibrium distance due to static repulsion and thus, increase the total molecule density on the interface. This is the reason for the decreasing interfacial tension after the CMC is reached. The pendant drop measurement provides values for $\gamma$ between 1.5 and $6 \mathrm{mN} / \mathrm{m}$.

We conclude from the ratios $\Delta \gamma / \gamma$ that a change in interfacial tension across the droplets circumference on the order of $10^{-4}$ of the equilibrium situation is sufficient for droplet propulsion in the range of $5-25 \mu \mathrm{m} / \mathrm{s}$. These minor values are in good agreement with the basic assumptions for Marangoni-powered swimmers (see Sec.2.3).

\subsubsection{Effect of chemotaxis}

In Sec. 2.1.1, the effect of chemotaxis on biological swimmers like E.coli was discussed. This behaviour is used to reach nutrient or light sources in an efficient manner. The same biases were also observed for surfing swimmers on interfaces [159, 160] or in Janus particles under illumination of laser light [16]. Following the discussion of the model system in Sec. 2.3 and the role of advection of empty micelles for the propulsion mechanism, it seems reasonable that the LC droplets are sensitive to global surfactant gradients. In this system, the attractants are the empty micelles, similar to a nutrient source.

To test whether chemotactic behaviour is present in LC droplets, we used a reservoir, millimetre sized in diameter and height, and a macroscopic amount of solid surfactant on one side of the reservoir (see Fig. 5.6). In this specific case, the background surfactant concentration was high enough for droplet propulsion. Qualitatively, the bulk surfactant starts to dissolve into the solution and creates an initial concentration of approximately $40 \mathrm{wt} \%$. Thus, a concentration gradient, pointing away from the source, evolves. Hence, the LC droplets move along this gradient. 


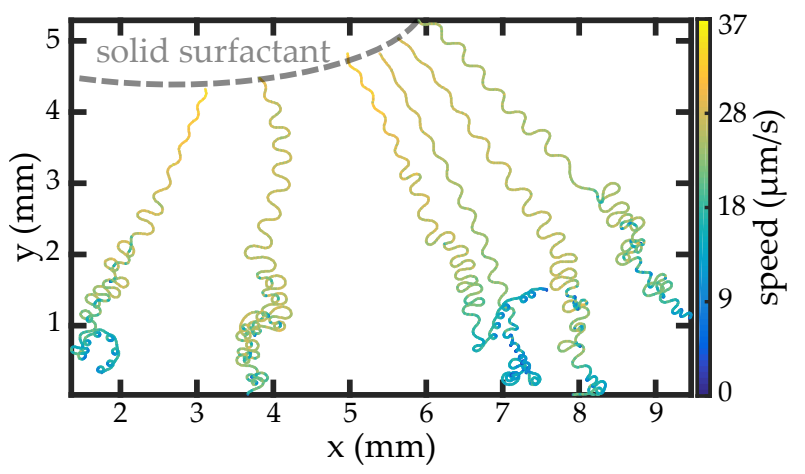

Fig. 5.6: Experiment highlighting the chemotactic behaviour of swimming LC droplets. A macroscopic amount of solid TTAB is inserted into a reservoir filled by a droplet suspension. The mean direction of the droplets follows the chemical gradient of the TTAB source. The movement of the droplets is accelerated until these reach the surfactant source. The colour code indicates the local velocity.
In Fig. 5.6, six representative trajectories are depicted. These were observed from the beginning of the experiment, implying the adding of solid surfactant, up to the point where the droplets reached the source. Far away from the source, each droplet propels with rather constant velocity (see colour code) until the gradient at the droplet's position is strong enough. Then, the droplet moves into the direction of the surfactant source, while its motion is even accelerated along the gradient. The superimposed curling motion in the early stages of the experiment is discussed in detail in Sec. 5.2.5.

The chemotactic behaviour is furthermore exploited in a second qualitative experiment. The latter is inspired by artificial maze solving systems [161, 162], usually considering oil droplets in $\mathrm{pH}$ - or salt gradients. A sub-millimetre sized maze, with channel widths of $200 \mu \mathrm{m}$ and a shortest path through the maze of $6 \mathrm{~mm}$ in length, is fabricated in PDMS. Two reservoirs serve as start and finish point for the droplets. The maze is filled with a background solution above the critical propulsion concentration. In particular, $c_{s}=12.5 \mathrm{wt} \%$ TTAB. The start reservoir contains droplets of $100 \mu \mathrm{m}$ in diameter and the finish reservoir contains the solid surfactant.

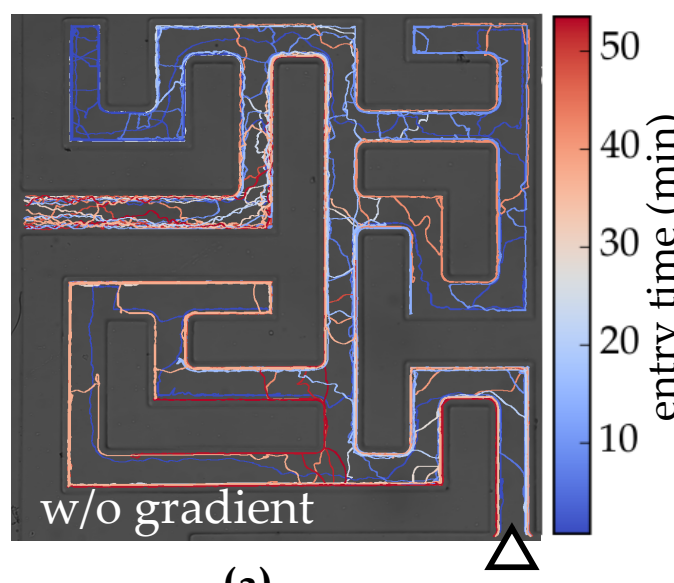

(a)

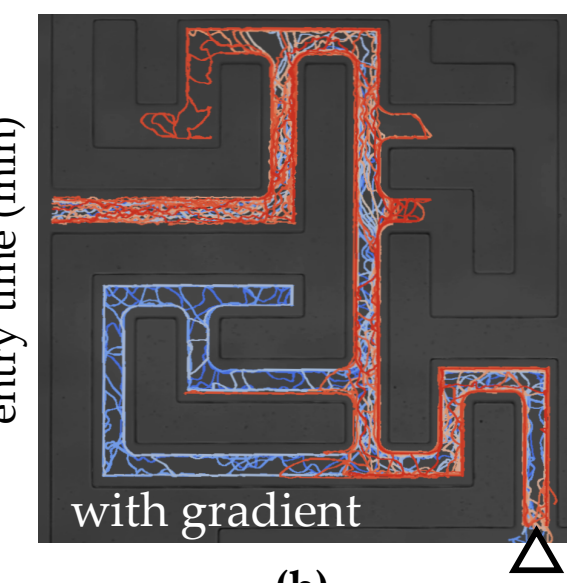

(b)

Fig. 5.7: Maze-solving experiment of chemotactic LC swimmers. (a) Experiment without and (b) with concentration gradient present. The arrowheads emphasize the entrance into the maze. The tracks are colour coded for displaying the entrance time into the maze. For intermediate times, the gradient provides a sweet spot for solving the maze along the shortest path, while in the control case, no distinct difference for varying starting times is observed. 
Motion is recorded over a course of roughly $60 \mathrm{~min}$. Droplets will start to invade the maze and move randomly through it. When a gradient is present (Fig.5.7p), a distinct maze solving is observed. In the control experiment (no gradient present), randomized motion of droplets occur (Fig. 5.7 ). Obviously, some of these droplets solve the maze even without the gradient, either by pure statistics or by rectification effects along the channel walls (see Sec. 5.2.4 for a discussion of wall effects).

However, a distinction between the gradient and control experiments can be made when comparing the trajectory length for maze solving. Therefore, the starting time, denoting when the droplet moves from the reservoir into the maze (see Fig. 5.8), needs to be considered. For early times (blue trajectories in Fig. 5.7), random motion is observed for both cases until, at intermediate times (20-40 $\mathrm{min})$, a temporal sweet spot for the gradient experiment emerges (white trajectories). In this regime, the gradient has fully evolved along the optimal path within the maze and droplets solve it nearly without deviations. Subsequently, additional surfactant saturates the side branches and a small increase in path length is observed (red trajectories) since droplets

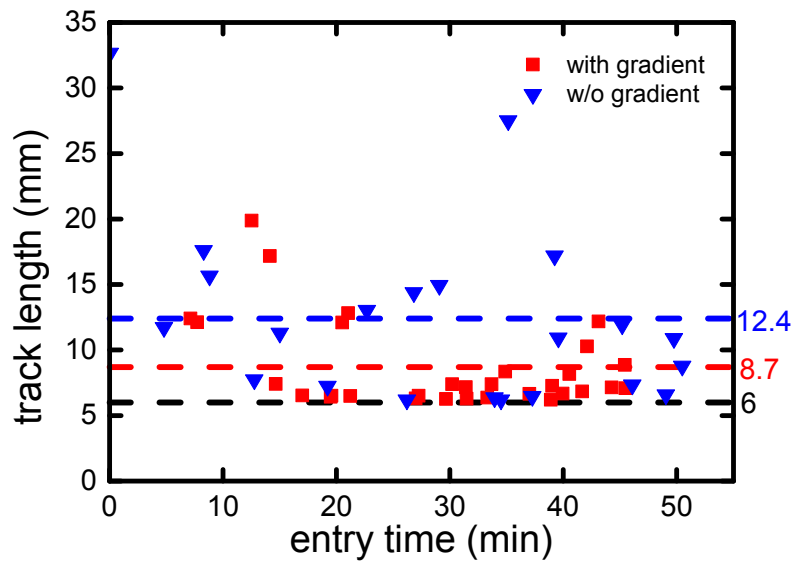

Fig. 5.8: Analysis of trajectory length of maze solving droplets. Here, the track length within the maze vs. the starting time is depicted. In the control experiment (blue triangles), no trend is observed. However, in the gradient experiment (red squares), after a short "incubation" time, paths close to the perfect solving are taken. This clearly shows the evolved surfactant gradient along the shortest path, which results in a substantially lower mean path length as compared to the control experiment. Dashed lines emphasize the mean track length of the measurement with a gradient present (red), without a gradient (blue) and the optimal path length (black). are misguided by multiple areas of higher surfactant concentration. Quantitatively, this results in average track lengths of $8.7 \mathrm{~mm}$ with a gradient present and $12.4 \mathrm{~mm}$ without a gradient (see Fig. 5.8).

Hitherto, only attractive forces generated by external biases have been discussed. However, in nature, also self-generated influences are present [163], especially for signal transfer in large groups of similar entities. This behaviour is called autochemotaxis, if the entities produce a chemo-attractant or -repulsive with which the entities themselves or others in the suspension interact. Positive autochemotaxis has been reported on in simulations [164-166], resulting in circular trajectories that depend on the interaction strength of the swimmer with the produced attractant. In the case of the LC droplet swimmer, however, one would expect the opposite case, namely negative autochemotaxis. In previous observations, we took the ex- 
ternally added empty micelles as a nutrient source into account. In this scenario, the filled micelles are rather considered as repellents. This results in avoidance of areas that have been beforehand passed by the same or another droplet for times on the order of tens-of-seconds.

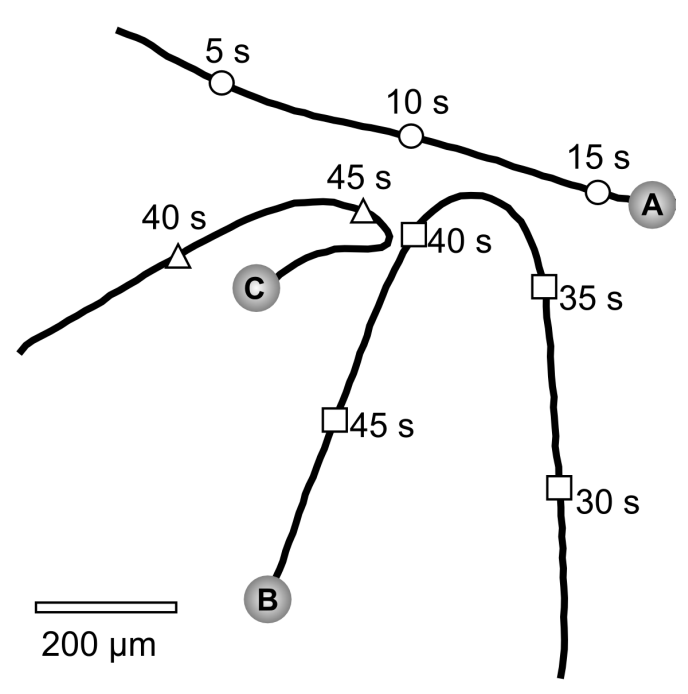

Fig. 5.9: Trajectories for droplets with autochemotactic repulsion. The symbols depict three different freely moving droplets, while the numbers written along the trajectories describe the temporal position of the locations. The two swimmers ( $B$ and $C$ ) are repelled by the trail of the beforehand passing swimmer (A). Figure provided by C. Jin, personal communication.
In simulations, this behaviour is as well shown in Brownian dynamics [167]. Here, the resultant ballistic motion at short times changes to diffusive behaviour on long terms. These interactions depend on the sensitivity of the droplets, the solubility and the diffusivity of the partaking species. The first notion of autochemotactic behaviour of the LC droplets appeared when droplets were observed at elevated temperatures, exceeding the nematic-to-isotropic transition. Here, the droplets move on rather ballistic trajectories and dissolve faster than at ambient conditions, such that more material is deposited in a trail behind the droplet. Thus, it occurs that droplets get repelled by trajectories of other recently passed droplets. This phenomenon is depicted in Fig. 5.9. The different symbols represent single swimmers and the numbers written along the trajectory mark different time stamps. It is clearly visible that the two droplets (B and C, around $40 \mathrm{~s}$ ) get repelled in the area that has been passed by a third droplet (A) within the first $15 \mathrm{~s}$ of recording. In this specific case, the experimental conditions were $c_{s}=7.5 \mathrm{wt} \%$ and $\mathrm{T}=40^{\circ} \mathrm{C}$ for $50 \mu \mathrm{m}$ sized droplets.

To visualize the deposited material in the trail of the droplet, we performed experiments at high surfactant concentrations and high temperatures. The idea is to show qualitatively how the droplet expels material and how the trail equilibrates by diffusion. For the respective measurements, we used $50 \mu \mathrm{m}$ sized droplets at $c_{s}=25 \mathrm{wt} \%$ and $T=35^{\circ} \mathrm{C}$, slightly below $T_{N I}$. Images are recorded in phase contrast microscopy at $10 \times$ magnification. Fig. 5.10 depicts three exemplary micrographs with the retrieved data below. Qualitatively, expulsion of material in the continuous medium is visible due to refractive index changes by 5 CB molecules. Material is expelled along the entire surface and is transported to the back of the droplet by surface flow. The micrographs in the top row show, with time differences of 10 and $40 \mathrm{~s}$, the evolution of the trail. In the first two images, the expulsion is visible, as well as the generated trail along the droplet's trajectory. The last image shows the maximum extent in time 

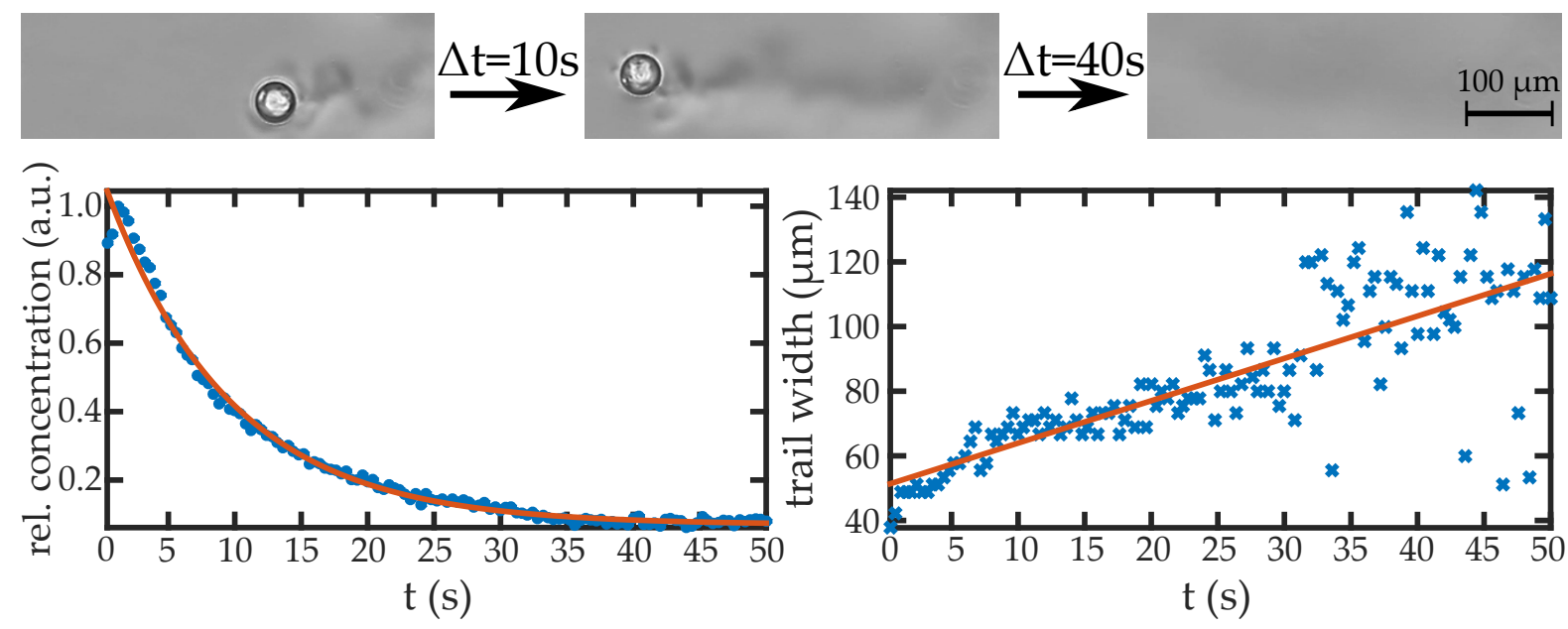

Fig. 5.10: In phase contrast microscopy, the temporal evolution of the $5 \mathrm{CB}$ concentration deposited in the trail is investigated. The top row shows three different micrographs, covering the whole duration of analysis. The first two pictures show the emerging trail of a passing droplet, while the last picture shows the equilibrated region $40 \mathrm{~s}$ after passage. In the bottom row, two graphs depict the concentration (left) and the trail width (right) as functions of time. For the concentration profile an exponential decay with the constant $t_{d}=9.3 \mathrm{~s}$ is retrieved, while for the trail width, a linear relation with a slope of $1.3 \mu \mathrm{m} / \mathrm{s}$ emerges.

that is analysed in this experiment. Here, the droplet has already left the field-of-view and no remains of the trace are visible.

In the phase contrast image, the trail appears as bright and dark areas across the trail, allowing us to investigate two quantities. The first quantity is the difference in brightness, which correlates with the local concentration of $5 \mathrm{CB}$ molecules inside micelles. The second quantity is provided by the doubled spatial distance of the minima and maxima of the hue values, corresponding to the width of the trail. A decrease in brightness difference is observed over time, whereas the trail width increases with time. For the brightness, an exponential decay is observed, described by a decay constant of $t_{d}=9.3 \mathrm{~s}$. The width of the trail increases linearly with a slope of $1.3 \mu \mathrm{m} / \mathrm{s}$. The intercept of the fit to the trail width corresponds to the droplet diameter, as expected. Thus, the phase contrast images can shed some light on the diffusive behaviour of the deposited trace of the swimmers.

These findings were the starting point for a quantitative investigation of the swimmers' autochemotactic behaviour. For these investigations, two experiments were constructed (see Figs. 5.11 and 5.12.

In a first attempt, the wall attraction of droplets is exploited in PDMS-based reservoirs with pillars of varying diameter. Here, the droplets attach to the walls and will be guided back onto their own trajectories. Different behaviours emerge, depending on the diameter $D$ of the pillars. The used surfactant concentration is $c_{s}=7.5 \mathrm{wt} \%$ and BPD is added in a ratio of 1:10 to 

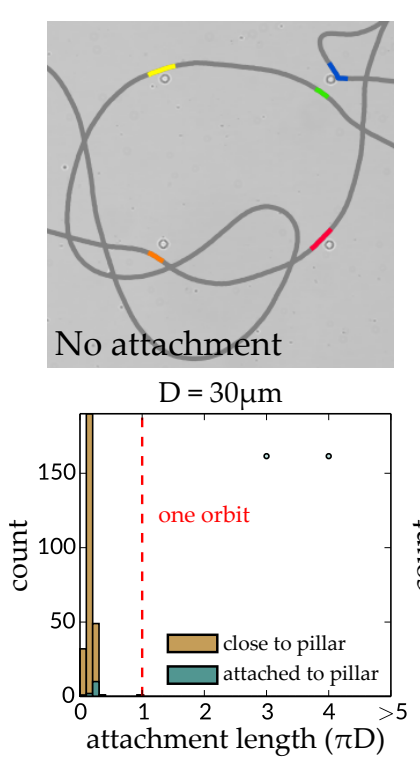
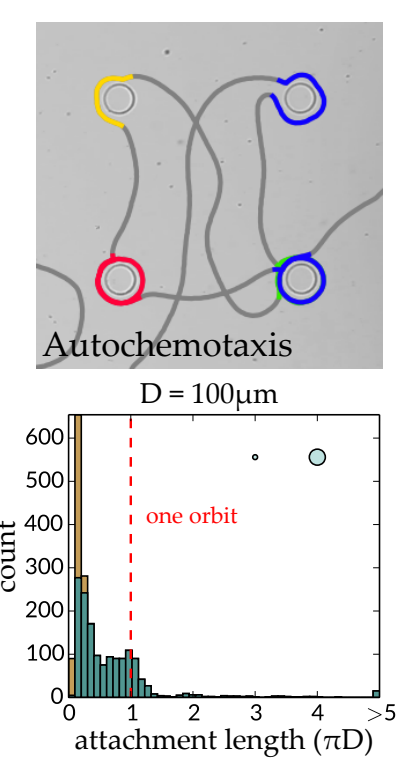
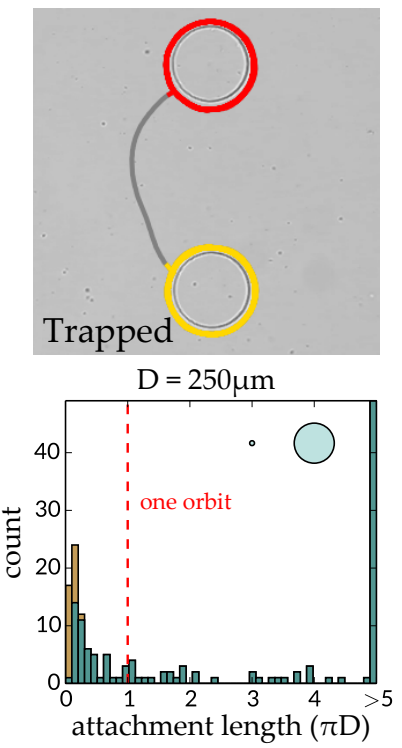

Fig. 5.11: Experiment for showing the self-generated autochemotactic behaviour of the LC/BPD droplets by trapping them at pillars with differing diameters. Top row, experimental micrographs and bottom row, histograms of the length of pillar attachment. Here, it is distinguished between the cases of trapped and colliding droplets. Figures provided by C. Jin, personal communication.

the $d=30 \mu \mathrm{m}$ sized droplets to switch off the nematic character. Fig.5.11 shows experimental micrographs in the top row and resultant histograms of the attachment length in the bottom row. In the histograms, it is distinguished between two different types of incident: first, the case when a droplet collides with but bounces off the pillar (yellow bars) and second, the case when it attaches to the wall of the pillar (green bars).

If the pillar diameter is small $(D \approx d)$, it is not possible to trap droplets along the circumference and thus, only droplet collisions with the pillar are observed. The reason for this is the high curvature of the obstacles. For large diameters, $D=250 \mu \mathrm{m}$ in this case, the droplets get trapped and revolve along the circumference of the pillar without any autochemotaxis visible. The orbital periods are long enough to disperse the autochemotactic trail by diffusion and the convex curvature of the pillar is low enough to be easily followed by the droplet. In an intermediate diameter range, e.g. $D \approx 100 \mu \mathrm{m}$, the curvature is low enough for droplets to get trapped but the transition time of one revolution is not large enough for equilibration of the bulk fluid. Thus, a significant number of droplets detach from the pillar after one orbit due to autochemotaxis. This is reflected by a peak in the histogram (see Fig. 5.11, middle) at an attachment length of one.

The second experiment consists of a row of pillars in a straight channel, modelling a series of binary junctions in a microfluidic device. Again, the channels are made from PDMS (see Fig. 5.12, top). Here, the retarded interaction of a droplet pair is investigated. The surfactant concentration is $7.5 \mathrm{wt} \%$ and the droplet's diameter is $50 \mu \mathrm{m}$, adjusted to the channel width. 


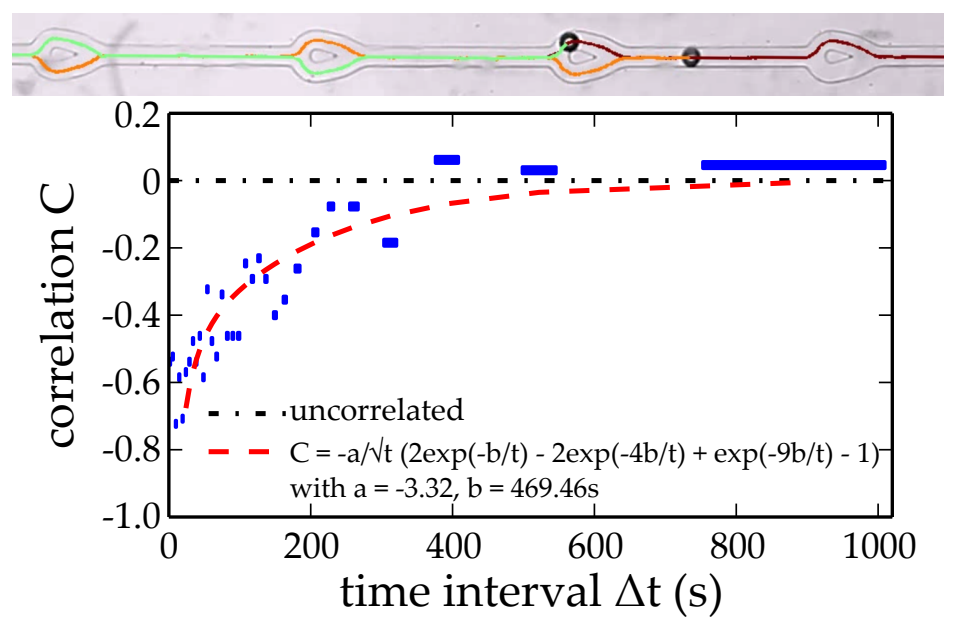

Fig. 5.12: Autochemotactic behaviour of the LC/BPD droplets in a branching channel. (Top) Experimental realization. (Bottom) Correlating the behaviour of a pair of droplets with various temporal distances resolves the exponential decay of anticorrelation. Blue bars emphasize the extend of the bin with the mean value as its centre [168].

The pillars are tear shaped to prevent droplets from turning back and rotating around the obstacle.

If the inter-passage time $\Delta t$ of two droplets is small, the second droplet will avoid the branch taken by the leading one. For large time differences, diffusion is able to equilibrate the micelle distribution around the pillar and no preference in path choice is observed. This time-dependent behaviour can be nicely visualized by a correlation of the droplets tracks in the vicinity of the junctions. Here, a strong anti-correlation is observed for small $\Delta t$. The anti-correlation decreases exponentially for increasing $\Delta t$. The time scale of the anti-correlation regime is on the order of $470 \mathrm{~s}^{-1}$ (Fig. 5.12, bottom) [168].

The correlation times in the chamber experiments exceed substantially the decay times measured in the phase contrast experiment mentioned above. Thus, the high sensitivity of the droplets to chemical gradients is emphasized. So far, we were not able to quantify e.g. the micelle concentration or the gradient threshold for which autochemotaxis occurs.

\subsection{Dilute systems in two dimensions}

\subsubsection{Hydrodynamic flow profile}

The self-propulsion of swimmers is always accompanied by interface distortions and a generated flow. In Sec. 2.4 the basics for describing these hydrodynamic flow profiles have been introduced. With the technique of Particle Shadow Velocimetry, we are able to quantify 

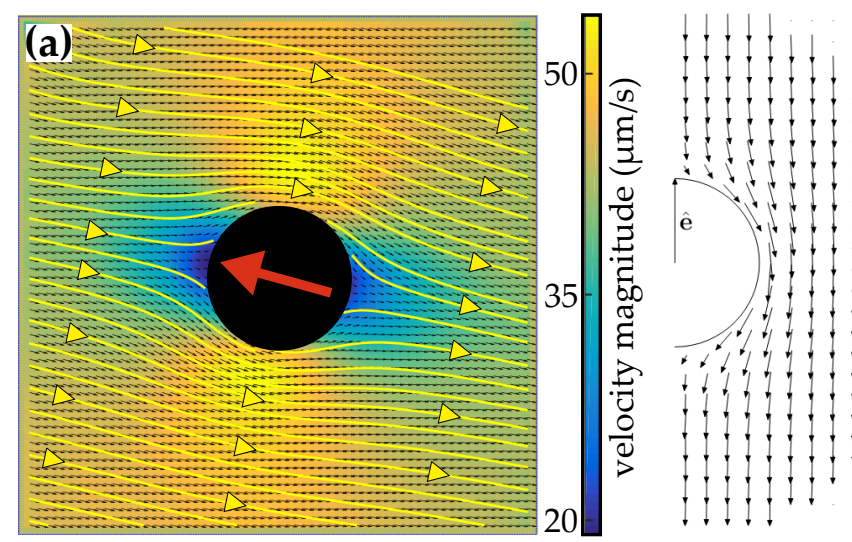

(b)

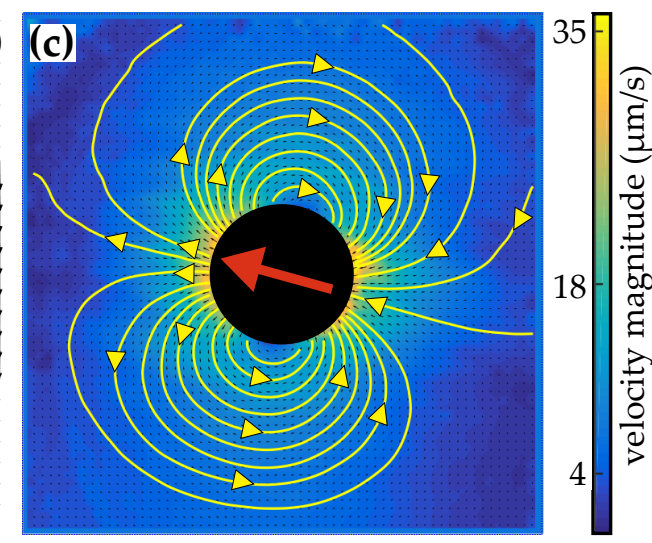

Fig. 5.13: Hydrodynamic flow profile. (a) Flow field in the droplet's co-moving frame of reference. (b) Simulated flow field for neutral squirmers, $\beta^{\prime}=0$, in the same frame as (a). Taken from [82]. (c) Flow field in the laboratory frame of reference. The red arrow emphasizes the propulsion direction, while the yellow arrows point in the flow direction of the outer liquid.

the flow profiles of the LC swimmers experimentally. In Fig. 5.13, typical results of such a measurement are shown. In this specific case, $60 \mu \mathrm{m}$ sized droplets were imaged with a $60 \times$ objective lens at a frame rate of $50 \mathrm{fps}$ with a high-speed camera. $1 \mu \mathrm{m}$ sized polystyrene colloids are used as tracer particles. We used a mixture of 10:1 LC/BPD as droplet material for switching to the isotropic state at room-temperature. The resultant suppression of curling motion will be discussed in detail in Sec.5.2.5. The analysis of the data follows the description in Sec. 4.6,

In the co-moving reference frame (Fig. $5.13 \mathrm{a}$ ), the streamlines bend around the droplet with the maximal velocity at the droplet's equator and stagnation points at the droplet's poles. This is in accordance with the schematic in Fig. 2.12. Since PSV is used as a measuring technique, limitations in resolution close to the droplet surface are inherent. This results in non-continuous streamlines at the droplet's poles. The obtained flow profile is similar to that of a neutral squirmer with $\beta^{\prime}=0$ as simulations, e.g. in [82] (see Fig. 5.13 ), suggest.

By subtracting the mean $x$ - and $y$-velocities, we transform the system into the laboratory frame (Fig. 5.13 ). Here, the streamlines start from the leading pole of the droplet and end at the rear, showing the typical butterfly pattern for $\beta^{\prime}=0$ squirmers.

Through plotting the tangential velocity along the droplet's surface (see Fig. 5.14a), we obtained a sinusoidal profile $(\alpha \sin \theta)$. This behaviour is in accordance with Fig. 2.13 for $\beta^{\prime}=0$ or with observations described in Ref. [79]. The oscillatory course is expected in the case of spherically symmetric Marangoni powered swimmers. When plotting the velocity at the equator $\left(\theta=\pi / 2=90^{\circ}\right)$ versus the relative distance $r / R$ from the droplet's interface, a decrease in magnitude is noticeable (see Fig. 5.14 $\mathrm{p}$ ). For $2 \mathrm{D}$ flow profiles, a $1 / r^{2}$ dependence is expected [169] as emphasized by the dashed line. Our experimental data are in good agree- 

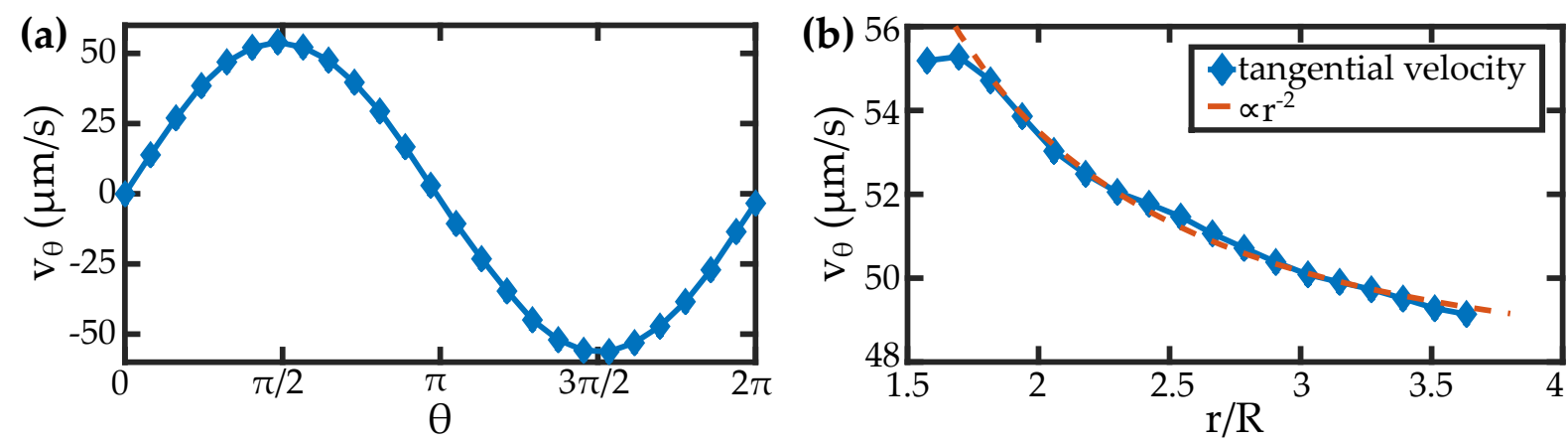

Fig. 5.14: (a) Tangential velocity $v_{\theta}$ vs. angle $\theta$. The proportionality of the surface velocity to $\sin \theta$ can be observed as expected. (b) $v_{\theta}$ vs. the relative distance $r / R$ from the droplet's surface with the droplet radius $R$. The expected relation of $\propto r^{-2}$ is emphasized by the orange dashed line. As can be seen from the $x$-axis in (b), the maximum distance is still in the vicinity of the droplet.

ment with the theoretical expected course. Deviations for small $r / R<1.7$ result from the aforementioned resolution limitations in the vicinity of the droplets. Furthermore, the $1 / r^{2}$ dependence is valid for the far field of the flow profile. Due to experimental restrictions, however, a maximum distance of four radii is achieved. This distance is still in the transition region between the near and far field.

With the hydrodynamic flow measurements, it is also possible to observe the interactions of the swimming droplet with colloidal tracers. This tracer scattering at low Reynolds numbers can provide valuable information about biological systems where nutrient uptake can also be described as interactions of motile swimmers with advected non-motile particles. This is studied experimentally in the case of Chlamydomonas rheinhardtii [170]. Here, an increase in diffusivity of tracer particles in bacteria suspensions is observed that furthermore depends on the bacteria density [171]. In the case of force free swimmers in simulations, these interactions result in loopy trajectories of tracers [172] which were experimentally confirmed in Ref. [6].

We investigated these interactions for the LC system to verify the assumption of force free propulsion. While $200 \mathrm{~nm}$ sized tracers were used in Ref. [6], being subject to strong Brownian dynamics, the $1 \mu \mathrm{m}$ sized colloids used here undergo significantly less randomized motion. Fig. 5.15 a, depicts the trajectories of the tracers in the laboratory frame of reference. Closed loops can be seen in the vicinity of the droplet's transit region (grey area), while only a minor effect is observed for larger distances. Due to the limited recording time, the loops of many tracers, especially for those that are further away from the droplet's surface, appear unfinished. However, the general form of the trajectories suggests the formation of closed loops, as predicted in Ref. [172].

These closed loops can be furthermore linked to the flow profile in the laboratory frame depicted in Fig. 5.13. Here, arcs of streamlines are visible, which the tracers follow during the transit. The loop is finalized by advection into the direction of the droplet, followed by drag 
(a)

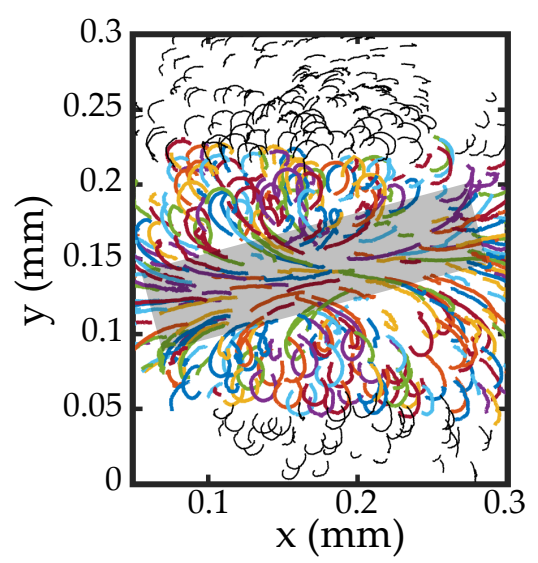

(b) $\quad x(\mu \mathrm{m})$

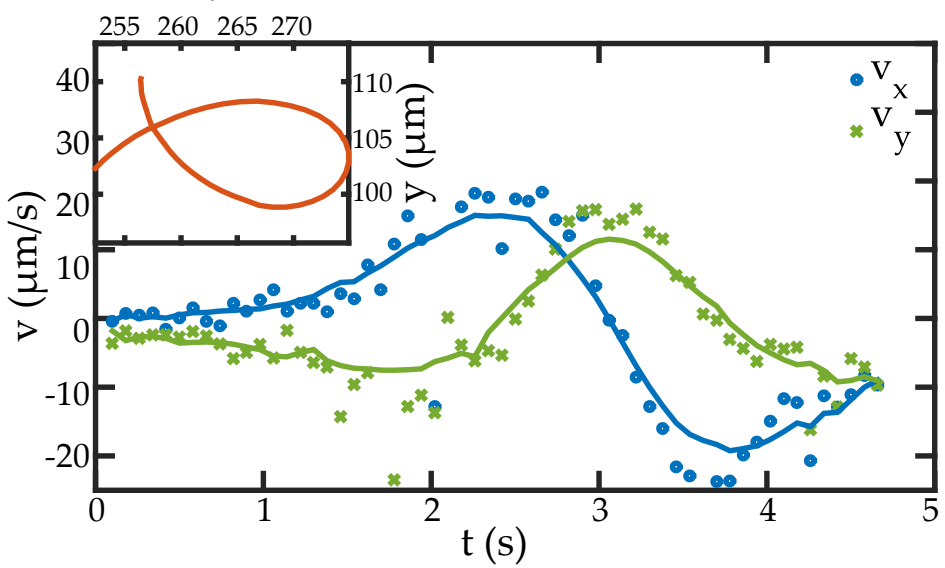

Fig. 5.15: Loopy tracer scattering by a force-free swimmer. (a) Interaction of colloids with the droplet. Loopy trajectories are observed in the laboratory frame. Grey area emphasizes the region passed by the droplet. Closed loops of tracers are visible in the vicinity of the transit region. (b) A single closed loop trajectory (inset), with the tracer velocity in $x$ - and $y$-direction.

when the droplet passed the tracer. In the co-moving reference frame, the tracers follow the streamlines that are shown in Fig. 5.13 . Hence, the two different approaches for flow-field analysis provide consistent results.

These interactions already indicate the influence of hydrodynamics on the droplet interactions, as we will see later on, and furthermore suggests a specific behaviour when droplets come close to walls (see Sec. 5.2.4).

\subsubsection{Diameter dependent velocity}

For experiments in two dimensions, we produced observation chambers by following the recipes described in Sec.4.1.2. Thereafter, a small number of droplets were mixed with the solution of desired surfactant concentration. The mixture was pipetted into the reservoir which was then closed by a cover slide (see Fig. 5.16a), resulting in a Hele-Shaw geometry. The small liquid layer between the edge of the reservoir and the cover glass results in minor changes in the total reservoir height but the initial evaporation of liquid seals the reservoir to prevent drift and further evaporation. Data is recorded by either bright-field or polarized microscopy. In both cases, the CCD camera provides recordings at frame rates between 4 and $25 \mathrm{fps}$. As a first step, velocities for droplets with initial diameters of $50 \mu \mathrm{m}$ were obtained for various surfactant concentrations (see Fig. 5.16p). The qualitative behaviour of the velocities is similar to the observations for the 1D case.

More interestingly, the additional degree of freedom as compared to the preceding 1D experiments is exploited. We measured the size-depending propulsion speed, since the ad- 


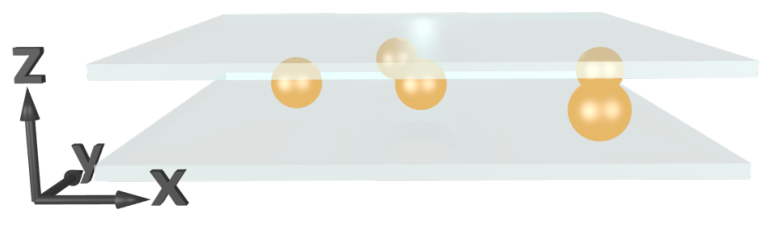

(a)

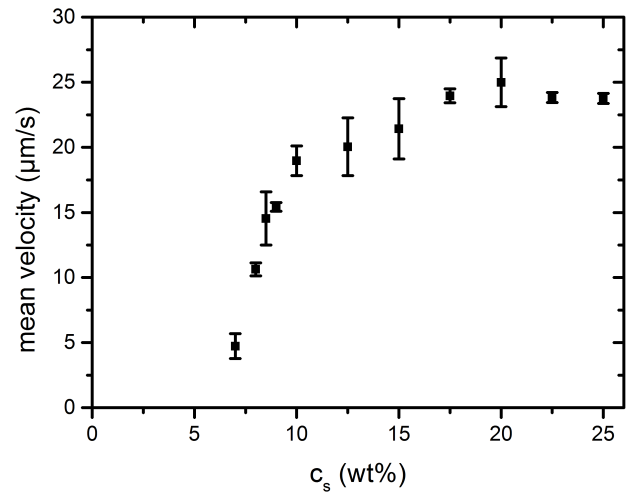

(b)

Fig. 5.16: (a) Schematic illustration of the Hele-Shaw geometry. The droplets are in a quasi-2D confinement and are observed via brightfield microscopy. (b) Mean velocity vs. surfactant concentration $c_{S}$ for droplets with $50 \mu \mathrm{m}$ in diameter. The qualitative behaviour is comparable to the 1D case. Figure adapted from [19].

vection-diffusion model presented in Sec. 2.3 predicts a size dependence (cf. eq. (2.11)). In Fig. 5.17a, data for four different surfactant concentrations, $c_{s}=10,15,20$ and $25 \mathrm{wt} \%$, with droplet diameters ranging from $8 \mu \mathrm{m}$ to $125 \mu \mathrm{m}$ are depicted. In both experiments, one- and two-dimensional, the velocities are in the range of $5-40 \mu \mathrm{m} / \mathrm{s}$. Interestingly, the mean velocity stagnates for large diameters at velocities of approximately $37 \mu \mathrm{m} / \mathrm{s}$, independent from the surfactant concentration. The velocity fluctuations in this regime can be easily explained by the internal flows. These need to be aligned for propulsion and start to get chaotic for large droplet volumes as compared to the ordered case that has been shown in Fig. 5.3. For droplets smaller than $60 \mu \mathrm{m}$, a linear increase in velocity is observed with increasing diameter, following a slope $m$ of $\approx 0.62 \mathrm{~s}^{-1}$. The latter is independent of the surfactant concentration and the behaviour is in qualitative agreement with eq. (2.11).

Using eq. 2.11 with appropriate parameters allows us to estimate the predicted velocity. The latter results from the approximations outlined in the theory section (cf. Sec. 2.3). As a reminder, we will start from eq. 2.11] with

$$
U=\frac{18}{4} \frac{\kappa R}{\delta\left(2+3 \eta_{d} / \eta_{c}\right)}
$$

For $5 \mathrm{CB}$ and TTAB, the viscosities are $\eta_{d}=\bar{\eta}_{1,2,3}=0.053 \mathrm{~Pa} \cdot \mathrm{s}$ and $\eta_{c}=0.0133 \mathrm{~Pa} \cdot \mathrm{s}$ [98], respectively. This results in the ratio $\eta_{d} / \eta_{c} \approx 3.96 . \kappa$ follows from Fig. 5.1 to $\kappa(10 \mathrm{wt} \%) \approx 5 \mathrm{~nm} / \mathrm{s}$. $\delta$ is the micelle size and is approximately $\delta \approx 2.5 \mathrm{~nm}$, assuming a spherical micelle instead of an elliptical one (see Fig. 3.1 for comparison). The droplet radius is chosen to $R=25 \mu \mathrm{m}$, since this size has been mainly used in these experiments. With these parameters, eq. (5.6) yields 


$$
U \approx \frac{18}{4} \frac{5 \mathrm{~nm} / \mathrm{s} \cdot 25 \mu \mathrm{m}}{2.5 \mathrm{~nm} \cdot(2+3 \cdot 3.96)}=16.2 \mu \mathrm{m} / \mathrm{s}
$$

This value is in good agreement with the values determined from the measurements shown in Figs. 5.2 a and 5.16b of $16 \mu \mathrm{m} / \mathrm{s}$ and $19 \mu \mathrm{m} / \mathrm{s}$, respectively. Furthermore, a merging of the constant parameters in eq. (5.7) simplifies eq. (5.6) to $U=c R$ with $c \approx 0.65$. This constant $c$ is in good agreement with the slope $m$ extracted from the experimental data. Thus, it is emphasized that the approximations in eq. (5.7) describe the experimental behaviour satisfactorily. However, the system's deviation from the linear behaviour at large droplet diameters cannot be modelled.

In active systems, one parameter is often used to compare systems of different origin with each other, namely the cruising range (CR). This parameter considers for how many diameters a droplet moves before the propulsion ceases. Thus, it can be described as

$$
\mathrm{CR}=\frac{\text { total distance travelled }}{\text { droplet diameter }}
$$

For our LC droplet system, this equation becomes slightly more complicated, since the droplet diameter and velocity changes over time. We therefore obtain

$$
\mathrm{CR}=\int_{0}^{t} \frac{v\left(t^{\prime}\right)}{d\left(t^{\prime}\right)} d t^{\prime}
$$

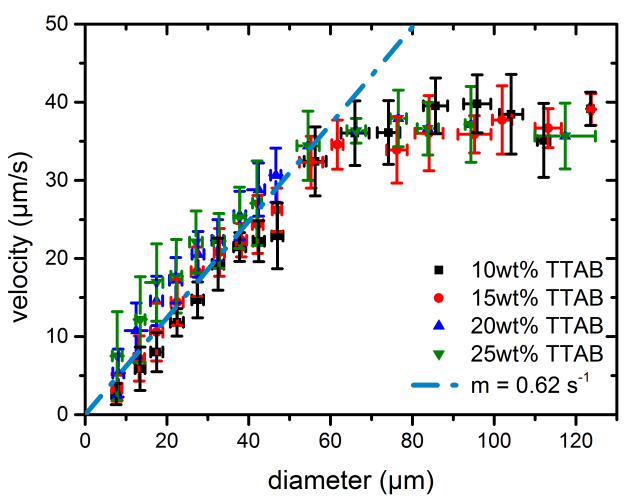

(a)

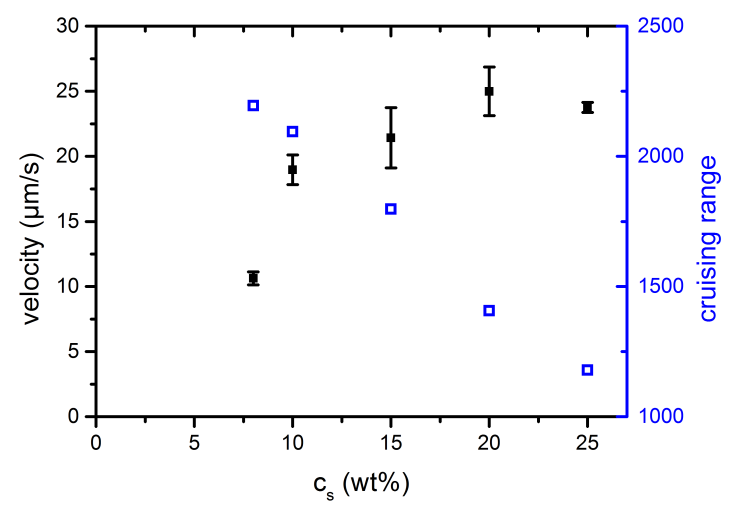

(b)

Fig. 5.17: (a) Velocity vs. diameter for four different surfactant concentrations, $c_{S}=10,15,20$ and $25 \mathrm{wt} \%$. The dash-dotted line emphasizes the linear increase in velocity with a slope of $m \approx 0.62 \mathrm{~s}^{-1}$. (b) Velocity for $d=50 \mu \mathrm{m}$ sized droplets (black $y$-axis) and cruising range (blue $y$-axis) vs. surfactant concentration. Values for the cruising range (blue open squares) are approximated with the gained $v(d)$ data and the solubility from Fig. 5.1. 
This equation is applied to different surfactant solutions $\left(c_{s}=8-25 \mathrm{wt} \%\right)$ and droplet diameters shrinking from $50 \mu \mathrm{m}$ to $10 \mu \mathrm{m}$. These parameters describe reasonable experimental conditions. The decrease in diameter and velocity is calculated from the aforementioned experimentally found values (see Figs. 5.1 $\mathrm{a}$ and 5.17 ) and compared to the mean velocity of droplets with a diameter of $50 \mu \mathrm{m}$ and two-dimensional confinement (Fig. 5.16 ). The results are shown in Fig. 5.17p. Even though the velocity increases with surfactant concentration, the cruising range decreases due to a non-linear ratio of solubilization and velocity. However, the cruising range takes values between 1000 and 2200 for all calculated cases. These values have been so far only obtained in very few active emulsion systems [18] or Janus-particle systems [16]. Many swimmer systems do not exceed a CR of a few hundred (see Tab. 1 in Ref. [19]). A large cruising range is beneficial for long term measurements, allowing to observe dynamics that occur on long time scales. In particular, some dynamics need time to evolve (see Sec. 5.4.1. A stability of the experimental conditions for such long times cannot be provided by systems with small cruising ranges.

\subsubsection{Movement threshold}

In Fig. 2.11b and Sec. 2.3. the importance of a critical surfactant depletion or, in other words, a critical driving parameter $\beta_{c}$ for the onset of locomotion has been discussed. In the case of $50 \mu \mathrm{m}$ sized droplets, the critical surfactant concentration is in the range of $6 \mathrm{wt} \%$. However, since the propelling speed is size dependent (see Fig. 5.17a), the propulsion threshold should be size dependent as well. Thus, the movement threshold for droplets with sizes of approximately $125 \mu \mathrm{m}$ is experimentally determined. For these sizes, a constant speed for the used surfactant concentrations was measured (cf. Fig. 5.17a).

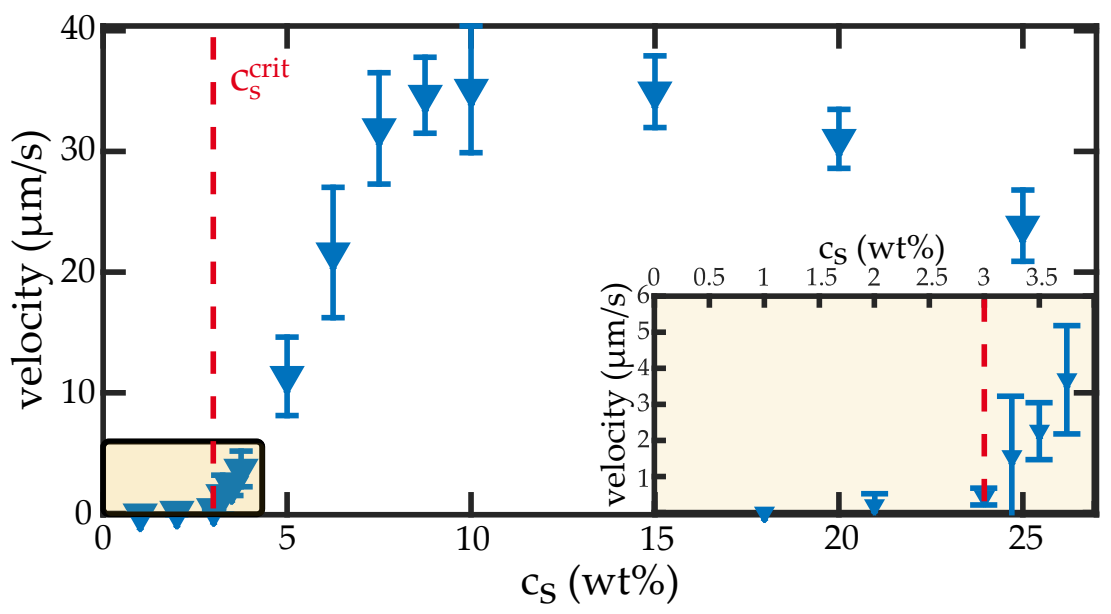

Fig. 5.18: Movement threshold for $125 \mu \mathrm{m}$ sized droplets in a $2 \mathrm{D}$ confinement. We determined the threshold concentration to $c_{S}^{c r i t} \approx 3 \mathrm{wt} \%$ (red dashed line), since a distinct velocity unequal from zero is measured for concentrations $c_{s}>3 \mathrm{wt} \%$. 
The mean velocity vs. surfactant concentration $c_{s}$ is depicted in Fig. 5.18. The inset emphasizes the low surfactant range between 0 and $4 \mathrm{wt} \%$. For small surfactant concentrations $\left(c_{s}<3 \mathrm{wt} \%\right)$, minor fluctuations in droplet positions in the processed data were visible. These fluctuations might result from Brownian motion or from pixelation effects in image processing. The mean velocities of the droplets, however, are not distinguishable from zero.

A distinct onset of locomotion is observed at $c_{s}=3 \mathrm{wt} \%$ with a non-zero velocity (red dashed line in Fig. 5.18 that increases with the surfactant concentration up to $35 \mu \mathrm{m} / \mathrm{s}$ at $15 \mathrm{wt} \%$. Thereafter, the velocity decreases with increasing surfactant concentrations $\left(c_{s}>15 \mathrm{wt} \%\right)$. Taking the obtained values and the left hand side of eq. (49) in Ref. [4] into account, a value for the critical driving parameter $\beta_{c}$ can be derived:

$$
\beta_{c}=\frac{c_{s}^{c r i t}-\mathrm{CMC}}{d / 2 \cdot \mathrm{CMC}}=0.35 \mu \mathrm{m}^{-1}
$$

\subsubsection{Wall interactions}

Active systems often show wall attraction and biological systems evolved strategies for biofilm formation with wall interactions as a key feature [173, 174]. Especially these interactions were reported on in flagellated swimmer systems, like E.coli or Chlamydomonas Reinhardtii. In E.coli, the interactions of near-wall hydrodynamics with the swimming entities generate circling trajectories [24]. In a subsequent study, these interactions were exploited in a microfluidic PDMS device. Here, the aligning behaviour of the entities on the right hand side of channels with respect to the propulsion direction has been shown [28]. Furthermore, E.coli shows accumulation of individuals close to walls [175]. Many more studies considering this effect have been conducted, focusing on e.g. the difference in interactions of pusher and puller type squirmer with straight walls [176], the inhibition of tumbling in E.coli near surfaces [32] or on the scattering and trapping behaviour of E.coli [177] or Chlamydomonas Reinhardtii [178] at obstacles with varying convex curvatures.

One of the first studies applying these wall interactions to passively maintain a rectification in non-equilibrium systems of bacteria is reported on in Ref. [179]. Here, the experimental chamber was divided by chevron-shaped obstacles. This arrangement induces a shape asymmetry to the system, such that an increase in density can be observed in one of the halves. This phenomenon is caused by hydrodynamic interactions and is therefore entirely physical in nature. Fig. 5.19 a depicts a sketch of how transitions from one to the other half are possible (track 1), while it is inhibited for other cases (tracks 2-4). The key feature is the guidance of swimmers along the walls. The interaction with one side of the chevrons allows transitions into the other half of the reservoir, while interactions along the other side of the chevrons capture the swimmers and inhibit the transition. 

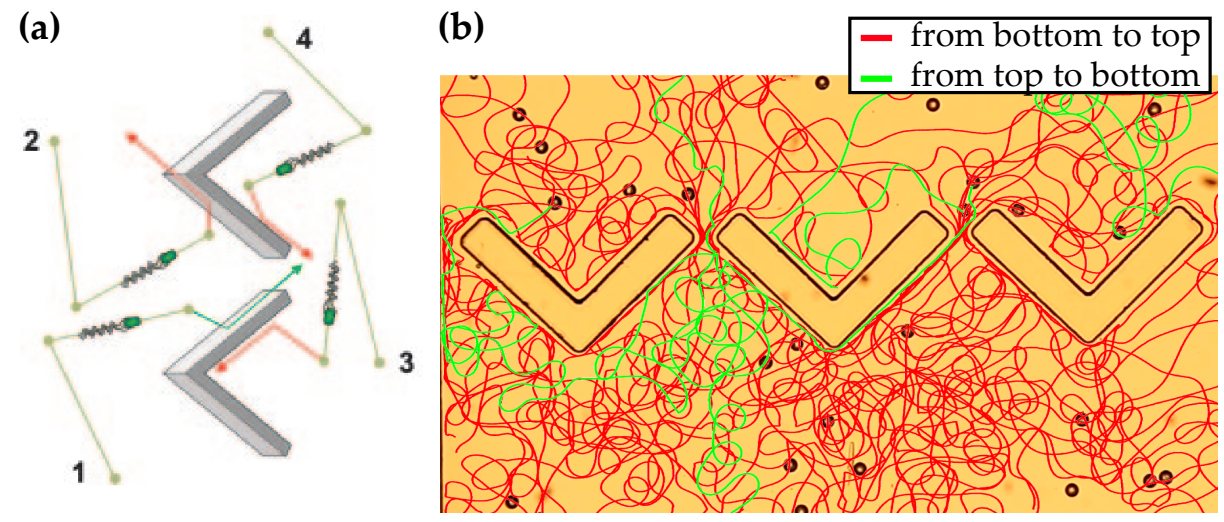

Fig. 5.19: (a) Schematic illustration of swimmer guidance due to wall attraction resulting in passive rectification. Track 1 results in a transition to the opposite half, while tracks 2-4 do not. Reproduced from [179]. (b) Experimental quantification of passive rectification. Green trajectories depict droplet transitions from the top to the bottom reservoir, while droplet trajectories highlighted in red point into the opposite direction. Droplets remaining in their respective halves are neglected. Of all droplets transitioning, $20 \%$ move to the bottom and $80 \%$ to the top, emphasizing the rectification.

This behaviour could be shown in simulations [180, 181], as well as in artificial experimental systems [6]. The ideas of rectification and sorting are further exploited in microfluidics, e.g. for E.coli [182] or in simulations with chiral swimmers [183].

We replicated the chevron setup in a test experiment for the LC droplets to emphasize the nonequilibrium behaviour of the system. Therefore, we used droplets with $50 \mu \mathrm{m}$ in diameter and chevrons with a gap of $75 \mu \mathrm{m}$ at a surfactant concentration of $c_{s}=10 \mathrm{wt} \%$ TTAB. The trajectories were recorded and subsequently analysed for droplets transitioning between the halves of the reservoir. In Fig. 5.19 b, a snapshot with the selected droplet trajectories is shown. Red trajectories depict droplets switching from bottom to top, while droplet trajectories shown in green follow a transition in the opposite direction. Trajectories of droplets remaining in one of the reservoirs are not highlighted. The red trajectories exceed the green paths substantially in number, which indicates a passive accumulation of droplets in the upper reservoir. Over the whole experiment, only 12 droplets transitioned to the bottom, while 50 droplets moved to the top. Thus, of all droplets transitioning, $80 \%$ moved to the top. This clearly emphasizes the rectification due to shape asymmetries.

After this rather complex geometry, we consider the simpler case of straight/curved walls. Simulations showed that swimmers align differently along these walls with detention times depending on the squirmer type [184-188]. The simulations indicated that especially pushers remain in the vicinity of walls, while neutral squirmers show a smaller tendency of attachment. Pullers align perpendicular to the walls and thus, get immobilized. Experimental systems showed wall hugging for artificial swimmers [6, 15, 189] with detention times linked to the persistence and rotation dynamics of the swimming entity. In these cases, the distribution of detention times mostly decays exponentially. 
The wall attachment is also observed for the liquid crystal swimmers in various settings. In Sec. 5.1.4, we already exploited the strong influence of wall attraction. Here, droplets even attached to convex curvatures. On straight walls and especially in circular observation chambers, droplets stay near a wall for a significant amount of time. A preliminary study will be discussed below.

In all the systems mentioned, the mechanisms for reorienting after capture are either due to rotational diffusion, which is rather negligible for the used droplets of $50 \mu \mathrm{m}$ in diameter (see Tab. 2.1), or due to inherent fluctuations as a result of the swimmers' properties. From the measurements of the hydrodynamic flow profile (see Sec. 5.2.1), we concluded that the LC droplets are of the neutral squirmer type $\left(\beta^{\prime}=0\right)$.

In the analysis scheme of the recorded data, attachment events are only counted as an incident if the droplet enters a region close to the boundary, defined by a threshold distance, and stays longer than $1 \mathrm{~s}$ in this region. Thus, elastic collisions are not counted, while minor distance fluctuations along the wall are still considered as one event. In particular, in the case of rectangular reservoirs, even $90^{\circ}$ turns are taken into consideration without splitting the event into two. This is necessary since straight walls longer than the attachment length are not observable within the spatial limit of the field of view at hand.

(a)

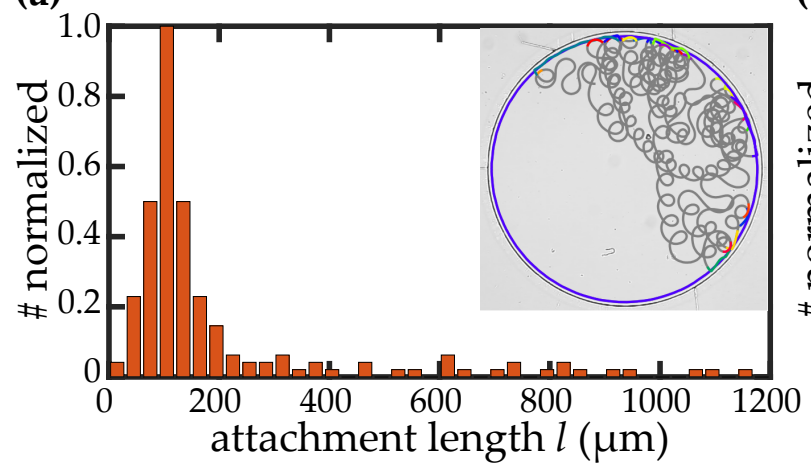

(b)

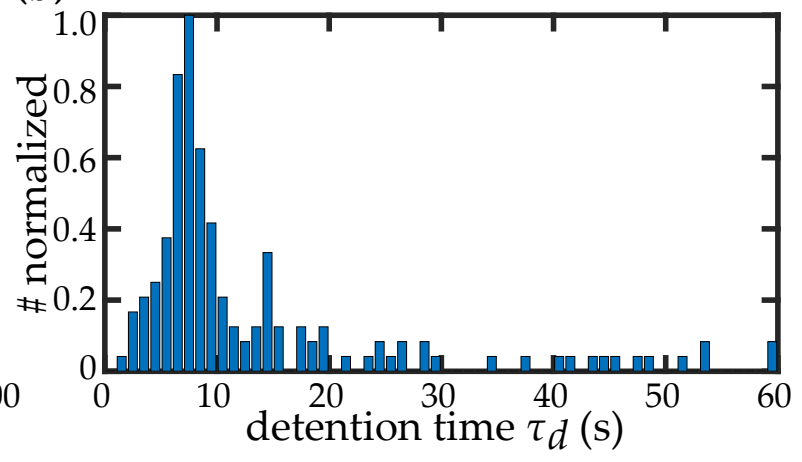

Fig. 5.20: Droplets of $d=50 \mu \mathrm{m}$ in circular confinement at $c_{s}=10 \mathrm{wt} \%$ and a reservoir diameter of $d=1.7 \mathrm{~mm}$. (a) Distribution of the attachment length $l$ at the walls with a typical trajectory as an inset. All coloured incidents are used for the analysis. Curling motion is observed inside the reservoir, which is completely ceasing at the wall. (b) Distribution of wall detention time $\tau_{d}$. The maximum values are obtained for $l_{\max }=105 \mu \mathrm{m}$ and $\tau_{d}=7.5 \mathrm{~s}$.

In the case of circular confinement $\left(D_{\text {res }}=1.7 \mathrm{~mm}\right)$, histograms of attachment length $l$ and detention time $\tau_{d}$ for $c_{s}=10 \mathrm{wt} \%$ TTAB are depicted in Fig. 5.20. The inset emphasizes a representative trajectory. Coloured instances mark parts of the trajectory within the threshold vicinity. Curling swimming is observed in the bulk reservoir but is inhibited at the wall. In total, 170 events are analysed and the distributions of both, attachment length and detention time, differ from an exponentially decaying distribution that has been observed for Janus 
particles [15]. The maximum of the distribution is at attachment lengths of approximately two droplet diameters $\left(l_{\max }=105 \mu \mathrm{m}\right)$. Moreover, the distribution exhibits a very long tail up to many tens of diameters ( $x$-scale not fully shown in the depicted histogram). The distribution for detention times shows a similarly behaviour with its maximum at $\tau_{d}=7.5 \mathrm{~s}$.

(a)

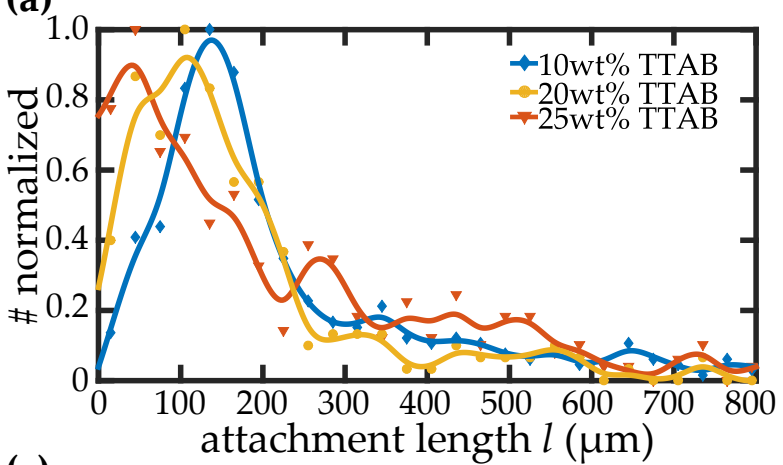

(c)

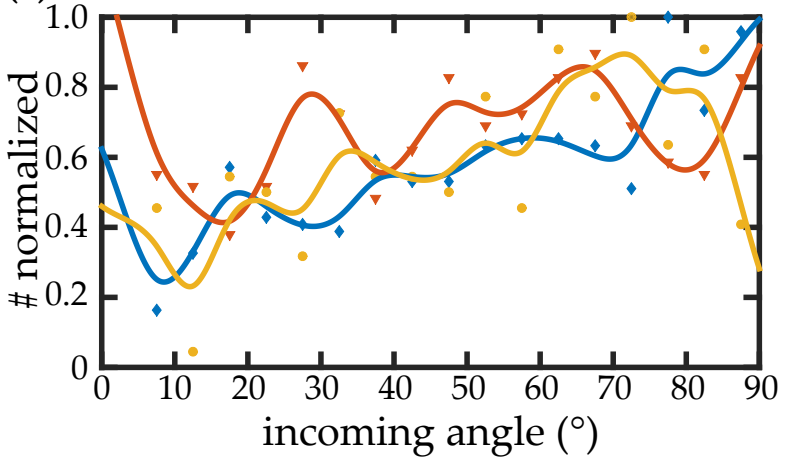

(b)

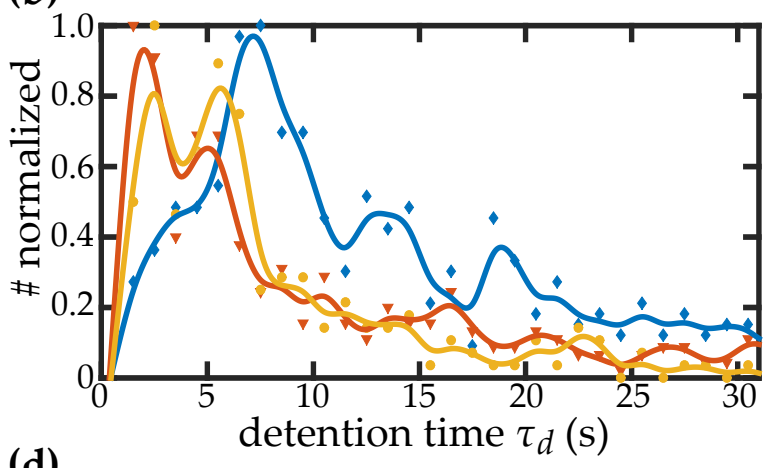

(d)

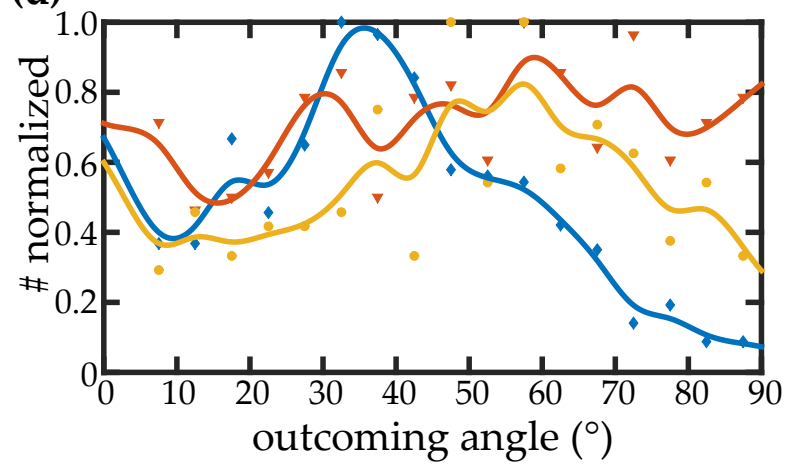

Fig. 5.21: Number of events for (a) attachment length $l$ and (b) detention time $\tau_{d}$ in the case of rectangular reservoirs in 2D and a droplet diameter of $50 \mu \mathrm{m}$. Both, the maxima for attachment length and detention time, decrease in magnitude with increasing surfactant concentrations, while the values for $c_{s}=10 \mathrm{wt} \%$ are comparable with the case of curved walls. (c) Depicts the incoming and (d) the outcoming angles for collision events. For $10 \mathrm{wt} \%$, the incoming angle shows a slight increase for perpendicular approach, while for the outcoming events, a hydrodynamic escape angle of $\approx 35^{\circ}$ emerges. Comparing the different concentrations, the distribution of incoming angles becomes broader, while the distribution of outcoming angles shifts to larger values with surfactant concentration. The colour code is the same in all four diagrams and a smoothing spline has been applied as a guide to the eye.

For rectangular reservoirs and thus, straight walls, the same quantities $l$ and $\tau_{d}$ are determined for 10, 20 and $25 \mathrm{wt} \%$ TTAB. Here, up to 500 events for each experiment have been considered (see Fig. 5.21a, b). For $10 \mathrm{wt} \%$ TTAB, the data sets for attachment length and detention time of curved and straight walls are comparable. When considering the different concentrations in rectangular reservoirs, however, the expected increase in the attachment length for higher velocities is not observed. Even contrary, the detention times decrease with higher weight percentage due to more frequent reorientation. In the case of $20 \mathrm{wt} \% \mathrm{TTAB}$, the velocity almost balances this effect with an attachment length $\left(l=v \cdot \tau_{d}\right)$ that is comparable to the $10 \mathrm{wt} \%$ case. For $25 \mathrm{wt} \%$, the attachment length decreases substantially. 
For straight walls, the incoming and outgoing angle for each event have been furthermore obtained (see Fig. 5.21k, d). Here, we consider the angle enclosed by the surface orientation and the propulsion direction of the swimmer. When analysing the data for $c_{s}=10 \mathrm{wt} \%$, intriguingly, there is no dependence of the maximal detention time on the incoming angle as mentioned in Ref. [188]. A rather flat distribution of incoming angles is observed, with a slight increase at high angles, that implies an approximately orthogonal incident. In this case, a droplet hits the wall head first and moves along the wall after a quick reorientation. For a smaller incident angle, the droplet slides along the wall without long delays due to reorientation. However, there seems to be a sweet spot for detaching at angles around $35^{\circ}$ with respect to the surface. This emphasizes an escape angle defined by the hydrodynamic interactions of the swimmers with the boundary.

Comparing the differing surfactant concentrations, a less distinct distribution for both angles is observed for 20 and $25 \mathrm{wt} \%$. For the incoming angles, one reason for the more flat distribution is the stronger hydrodynamic flow profile for faster droplets. Thus, a capture due to wall interactions is more likely independent of the incoming angle. On the same behalf, the escape angle due to increased hydrodynamic interactions should increase for faster droplets. Indeed, a slight shift of the distribution to larger outcoming angles is observed in the data.

For a more detailed analysis, further experiments are being planned for both confinements, circular and rectangular, and for varying parameters e.g. the reservoir diameter, surfactant concentration, droplet size and liquid crystalline phase.
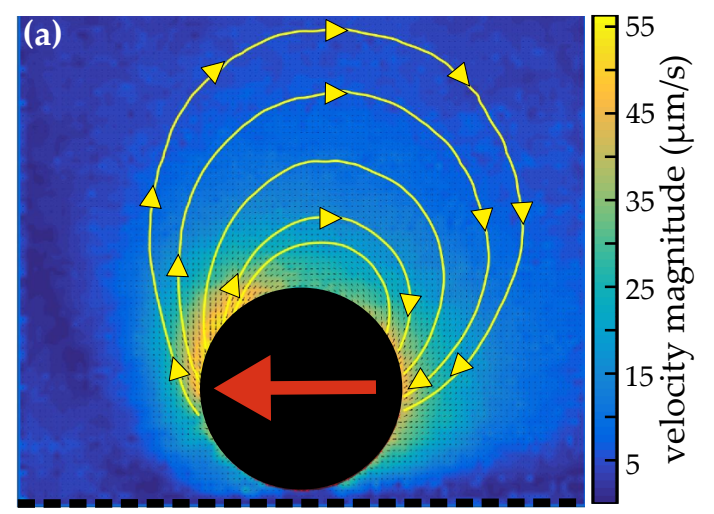

(b)

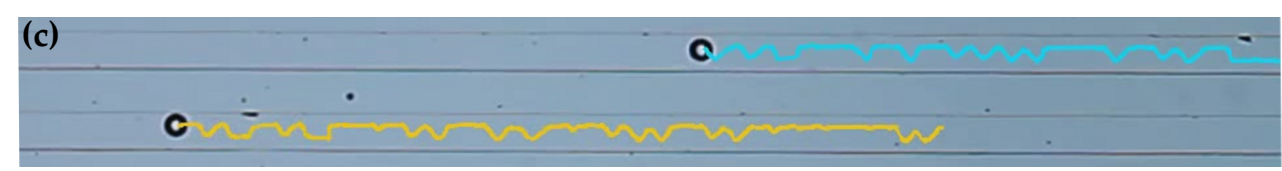

Fig. 5.22: Influence of confinement on droplet behaviour and flow field. (a) \& (b) PSV results in the laboratory frame obtained for $c_{s}=7.5 \mathrm{wt} \%$ TTAB and $85 \mu \mathrm{m}$ sized droplets. The wall-to-wall distances are 400 and $150 \mu \mathrm{m}$, respectively. The red arrow indicates the propulsion direction, yellow arrows depict the flow direction and black dashed lines indicate the boundaries. The distortion of the flow profile in the narrower channel is clearly visible. (c) Exemplary trajectories for wall switching droplets in narrow channels. Fig. (c) is provided by C. Jin, personal communication. 
In recent experiments, we observed that droplets start oscillating between two walls when the wall-to-wall distance is comparable to the droplet diameter. The basic idea for the following measurements is that the flow profile of the propagating droplet in the equatorial plane interacts with the opposing channel walls. When the droplet is close to the boundary, a back action on the droplet will occur. This results in symmetric interaction potentials between the droplet and either of the two channel walls that provide an oscillatory motion of the droplet. The analysis of this experimental setting leads to information about the strength and range of the droplet-wall interaction.

Fig. 5.22 depicts exemplary trajectories of $100 \mu \mathrm{m}$ sized swimmers in $150 \mu \mathrm{m}$ wide channels for a surfactant concentration of $c_{s}=7.5 \mathrm{wt} \%$. In these experiments, the nematic character of $5 \mathrm{CB}$ is switched off by admixture of $10 \%$ BPD. Thus, the droplets are prevented from curling swimming, as subsequently discussed in Sec. 5.2.5. We used PSV to visualize the interaction of the flow with the confining walls. TTAB solutions with $7.5 \mathrm{wt} \%$ were seeded with $500 \mathrm{~nm}$ sized polystyrene colloidal particles and droplets of $85 \mu \mathrm{m}$ in diameter were observed in different confining settings. Figs. $5.22 \mathrm{a}$ and $\mathrm{b}$ depict flow fields for

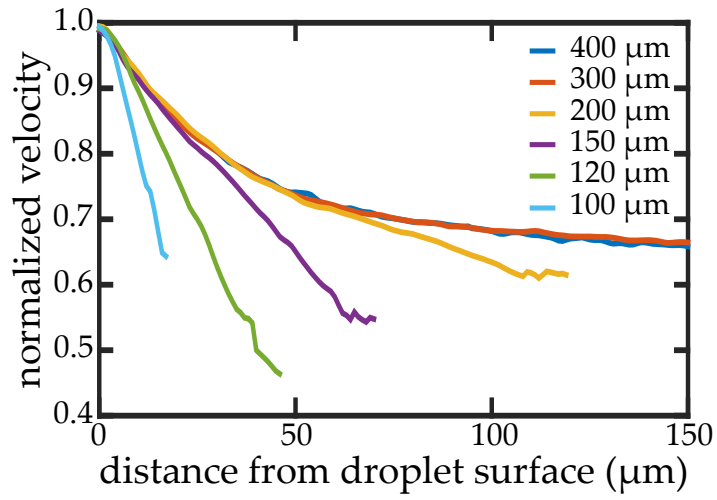

Fig. 5.23: Velocity profile analysed along the equatorial surface normal of the droplet. Data is shown for all tested confinements, starting from wall-to-wall distances of $400 \mu \mathrm{m}$ down to $100 \mu \mathrm{m}$, with a droplet diameter of $85 \mu \mathrm{m}$. wall-to-wall distances of 400 and $150 \mu \mathrm{m}$ in the laboratory frame. The droplet swims along one of the walls (indicated by black dashes with the propulsion direction marked by the red arrow) and the flow velocity profile is analysed in the azimuthal mid-plane of the droplet. The hydrodynamic flow profile is nearly undisturbed in the large channel, while it is strongly distorted due to the interaction of the flow profile with the upper wall in the smaller channels.

The influence of the confinement on the flow profile is even better emphasized when the normalized radial flow velocity vs. the distance from the droplet surface is analysed. The data along the equatorial surface normal for all tested cases with wall-to-wall distances between 100 and $400 \mu \mathrm{m}$ is depicted in Fig. 5.23. A difference in slope is clearly visible for different wall-to-wall distances, indicating the back action of the second wall onto the velocity profile. 


\subsubsection{Curling liquid crystal swimmers 1}

In Sections 2.1.1 and 2.1.2, different mechanisms for curling in 2D or helical motion in 3D in both, biological and artificial systems, caused by structural asymmetries were introduced. For bioswimmers the main reason for this behaviour is either a propulsion strategy or hydrodynamic interactions in the vicinity of boundaries [24, 31, 38]. In artificial systems, the necessary twofold structural asymmetry is examined in both, experiments and simulations [39, 42, 58, [59, 191-193].

Similar to the studies on biological and asymmetric artificial swimmers, a curling motion in two dimensions and helical trajectories in three dimensions for liquid crystal swimmers are observed. The important difference is that our system is spherically symmetric at rest. The necessary rotational torques are generated by the interplay of surface flow and nematic order. Thus, the curling motion can be switched off by heating the system to the isotropic state and the droplet reverts to persistent swimming. Since fundamental parameters and forces can be reproducibly tuned, this system is exceptionally suited for studying the curling motion.

In addition to hydrodynamic propulsion forces, the droplets are also sensitive to gradients in empty micelle concentration. Since each droplet deposits a trail of filled micelles behind, the mentioned sensitivity induces them to avoid each other's as well as their own trajectories (see Sec.5.1.4). Consequently, they show negative autochemotaxis [194] on time scales shorter than the required time for micellar diffusion. However, this autochemotactic repulsion is not strong enough to entirely suppress self-crossing trajectories.

In the curling case, trajectories in quasi-2D Hele-Shaw cells are analysed. Here, we varied the surfactant concentration $c_{s}$ between $7.5 \mathrm{wt} \%$ and $15 \mathrm{wt} \%$. Thus, the concentration is well above the $\mathrm{CMC}$ and above the propulsion threshold. Droplets of $5 \mathrm{CB}$ with an initial diameter of $50 \mu \mathrm{m}$ were injected into a quasi two-dimensional reservoir with an area of $10 \times 6 \mathrm{~mm}$ and a height of $h \approx 50 \mu \mathrm{m}$, prefilled with TTAB solution. Trajectories were recorded at $4 \mathrm{fps}$ under a bright-field microscope with a $2 \times$ objective lens.

Self-propulsion was quantified via the MSD (cf. eq. (2.19) with

$$
\left\langle\Delta r^{2}(t)\right\rangle=\left\langle\left(\mathbf{r}\left(t_{0}+t\right)-\mathbf{r}\left(t_{0}\right)\right)^{2}\right\rangle_{t_{0}}
$$

and the angular autocorrelation function $C(t)$ of the trajectories, given by

$$
C(t)=\left\langle\frac{\mathbf{v}\left(t_{0}+t\right) \cdot \mathbf{v}\left(t_{0}\right)}{\left|\mathbf{v}\left(t_{0}+t\right)\right|\left|\mathbf{v}\left(t_{0}\right)\right|}\right\rangle_{t_{0}}
$$

\footnotetext{
${ }^{1}$ The content of this section is published in: C. Krüger et al. Curling liquid crystal Swimmers: A Cascade of Spontaneous Symmetry Breakings, Physical Review Letters 117, 048003 (2016) [190]
} 

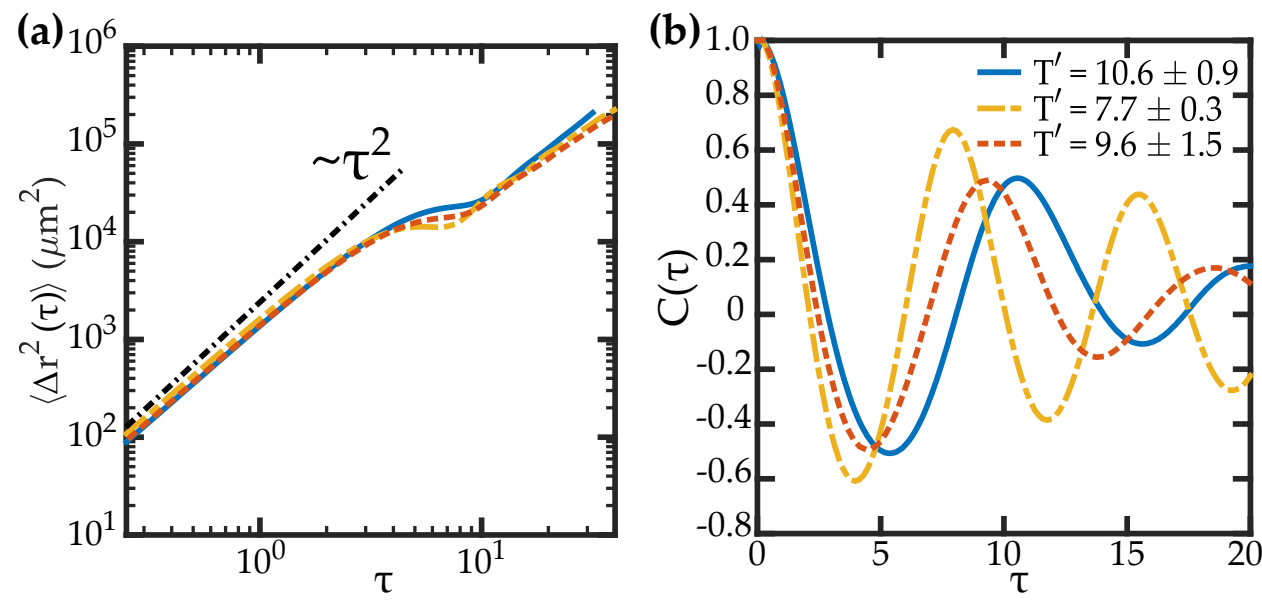

Fig. 5.24: (a) MSD and (b) angular autocorrelation $C(\tau)$ for experiments in the nematic state with $c_{s}=7.5 \mathrm{wt} \%$ at $22^{\circ} \mathrm{C}$ (solid), $30^{\circ} \mathrm{C}$ (dash-dotted) and $34^{\circ} \mathrm{C}$ (dashed). Through the rescaling of the MSD and the autocorrelation with respect to $\tau$, the data sets coincide up to one rotation period $T^{\prime}$.

As the droplets dissolve continuously, neither speed nor diameter are constant over time. These variations have to be considered for correlations in the long time limit (see Sec. 5.2.2. A simple method to derive a natural, unit free progressive scale $\tau$ for the system is provided by the accumulated trajectory length in units of the current diameter $d(t)$. In particular, $\tau$ can be obtained by integrating the ratio of the instantaneous speed $v(t)$ and current diameter $d(t)$ over time:

$$
\tau=\int_{0}^{t} \frac{v\left(t^{\prime}\right)}{d\left(t^{\prime}\right)} d t^{\prime}
$$

We have plotted the rescaled quantities $\left\langle\Delta r^{2}(\tau)\right\rangle$ and $C(\tau)$ (see Fig. 5.24) for a series of experiments taken at constant $c_{S}$ while increasing the temperature in the range below $T_{N I}$. This leads to an increase in swimming speed, while the curling motion is retained. Each data set contains between 12-16 trajectories, similar to those plotted in Fig. 5.25a, recorded over a time span of 4-5 minutes. Individual droplet sizes vary between 30 and $45 \mu \mathrm{m}$. Respective MSDs are plotted in Fig. 5.24(a).

The characteristic MSD for this type of swimmer shows a ballistic course which is proportional to $\tau^{2}$ for short times up to the rotation period. Here, we see a dip in the curve that is caused by the trajectory bending back on itself while curling. On longer time scales, the MSD recovers towards a slightly subballistic behaviour. When compared to the predicted MSD for active Brownian particles (see eq. (2.23) ) and experiments on Janus swimmers [15, 55], we note that, due to our swimmer's large size, their diffusion constant is substantially smaller $(D \approx 5 \times$ $\left.10^{-3} \mu \mathrm{m}^{2} / \mathrm{s}\right)$, whereas rotational diffusion times $\left(\tau_{r} \approx 60 \mathrm{~s}\right)$ are comparably long. Accordingly, we do not see diffusive contributions for very short times and only beginning deviations from 

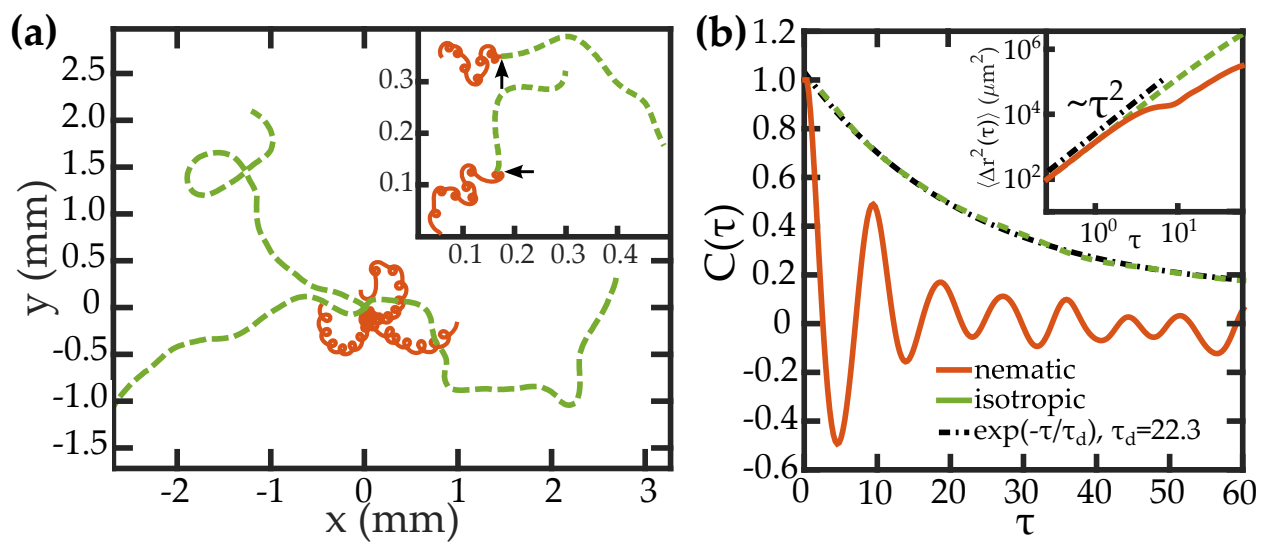

Fig. 5.25: (a) Example trajectories of $50 \mu \mathrm{m}$ sized droplets at $c_{s}=7.5 \mathrm{wt} \%$ over the course of $90 \mathrm{~s}$ at $34^{\circ} \mathrm{C}$ (nematic, solid line) and $37^{\circ} \mathrm{C}$ (isotropic, dashed). Above $T_{N I} \approx 35^{\circ} \mathrm{C}$, the curling is suppressed. Inset: Trajectories of $100 \mathrm{~s}$ duration with a temperature ramp across the nematic-to-isotropic transition. The black arrows emphasize the transition. (b) Comparison of angular autocorrelation $C(\tau)$ in the rescaled time frame $\tau$. An exponential fit to the isotropic data set of $C(\tau)$ results in a decay time $\tau_{d}=22.3$ (dash-dotted, black). Inset: Comparison of MSD $\left\langle\Delta r^{2}(\tau)\right\rangle$ for the curling and the isotropic case.

ballistic behaviour for long times, as evidenced by the high trajectory persistence beyond the regime of superimposed curling (Fig. 5.25 ). The rescaling to the progressive time scale $\tau$ yields a good compliance for data of ballistic, rotational and recovery regimes, with the characteristic dip at $\tau \approx 10$.

The angular autocorrelation is expected to be constant $(C(\tau)=1)$ for ideal ballistic swimmers and exponentially decaying $\left(C(\tau)=e^{-\tau v / L_{p}}\right)$ in the case of diffusive behaviour with persistence length $L_{p}$. In our system, the curling causes a characteristic superimposed oscillation $\propto \cos 2 \pi T^{\prime}$ with the rotation period $T^{\prime}$ of the swimmer. Rescaling to the new time scale $\tau$ is successful up to one period $T^{\prime} \approx 10$ (Fig. 5.24 ). This observation is consistent with the dip in the rescaled MSD.

As shown in Fig. 5.25a, the droplet trajectories show a pronounced curling when observed at temperatures below the nematic-to-isotropic transition (solid lines) of $T_{N I} \approx 35^{\circ} \mathrm{C}$. When heated above the transition (dashed lines), the curling motion ceases immediately (see inset in Fig. 5.25a) and the trajectory reverts to persistent swimming. The latter is nearly ballistic on the time scale of observation. We should note that no chiral component is present in our system and thus, clockwise and counterclockwise rotations are observed with equal probability.

The observed data sets indicate that the nematic ordering is necessary for the curling instability. In Fig. 5.25 , we show trajectories for the same surfactant concentration $c_{\mathrm{s}}=7.5 \mathrm{wt} \%$ at temperatures $\mathrm{T}=34^{\circ} \mathrm{C}$ and $37^{\circ} \mathrm{C}$, i.e. slightly below and above $T_{N I}$. The curling is entirely suppressed for $T>T_{N I}$. As soon as the transition temperature is reached, the curling motion changes to a smooth trajectory with exponentially decaying angular autocorrelation 

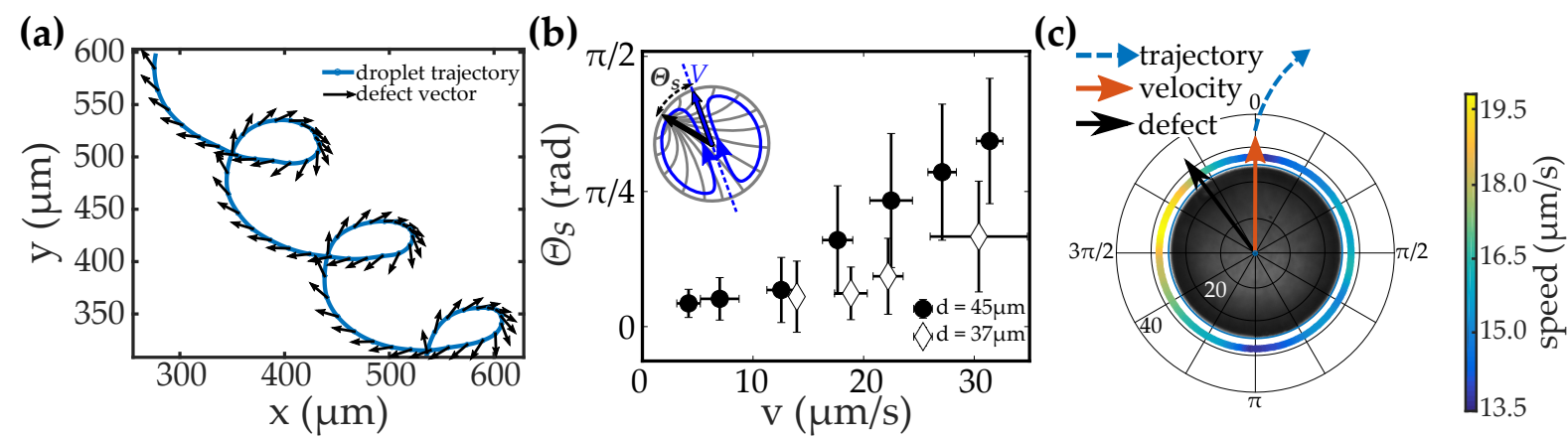

Fig. 5.26: (a) Curling trajectory of a $50 \mu \mathrm{m}$ sized droplet with depicted defect vectors (arrows) and swimmer trajectory (solid line). (b) Averaged phase shift $\Theta_{s}$ between velocity and defect vs. droplet speed for two droplet sizes. (c) Tangential velocity at a distance $\approx 4 \mu \mathrm{m}$ from the droplet interface. The flow is increased around the defect location.

(Fig. $5.25 \mathrm{~b})$, corresponding to a quite large persistence length $\left(L_{p} \approx 20 d\right)$. The characteristic dip in $\left\langle\Delta r^{2}(\tau)\right\rangle$ and the oscillation in $C(\tau)$ observed for the curling case are fully suppressed for isotropic droplets.

The underlying physical mechanism for the curling motion can be found by studying the nematic structure of a moving droplet between crossed polarizers. As discussed in Secs. 2.2 and 5.1.2, the surfactant layer at the surface of the droplet causes a homeotropic anchoring of the nematic director at the droplet interface. This anchoring results in a hedgehog defect in the centre of a resting droplet [195]. The internal convection of a self-propelling droplet advects this point defect towards the leading edge of the droplet (cf. Fig. 5.3 a).

Intriguingly, for curling motion in a two-dimensional reservoir, we always observe a finite angle $\Theta_{s}$ between the velocity vector and the defect vector. The latter points from the droplet centre to the defect location (Fig.5.26a and inset in Fig.5.26p). This deviation can be explained by the fact that the defect orientation towards the stagnation point at the droplet apex is not stable against fluctuations in the polar angle $\theta$, since the surface flow $v_{s}$ follows $v_{s} \propto \sin \theta$ to first order [79] (see Sec. 5.2.1. Accordingly, the defect is pulled away from the apex until this deflection is countered by elastic restoring forces that are caused by the deformation of the nematic director field. Since tangential stresses are continuous across the interface and there is no elastic restoring force in the aqueous phase, the droplet experiences a net tangential force. Hereby, the outer phase is pulled towards the droplet equator, resulting in a counterrotating torque acting on the droplet.

Microscopically, due to the homeotropic anchoring, the flow of the nematic material near the droplet surface depends on the Miesowicz viscosity $\eta_{2}$ (director parallel to the velocity gradient, see Fig. 2.8), which is the largest of the three nematic viscosity coefficients [72]. Close to the defect, however, the director is rotated away from the interface normal and the nematic order parameter decreases. This causes the effective viscosity to decrease towards the 
(a)

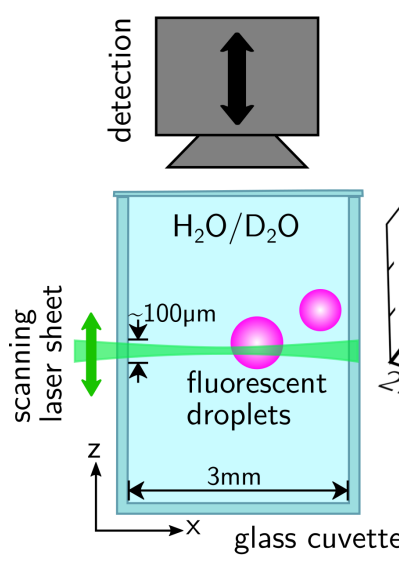

(b)

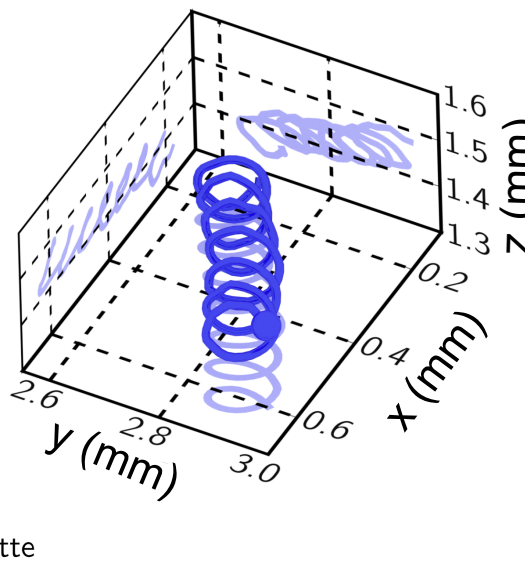

(c)

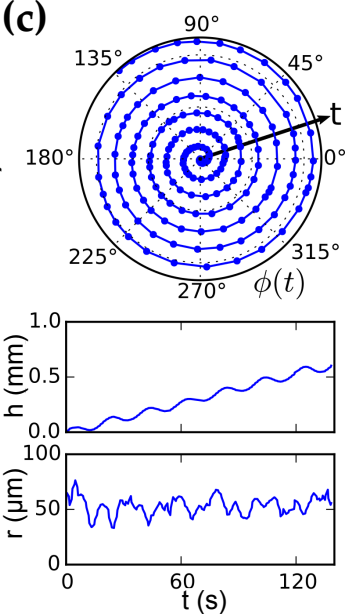

Fig. 5.27: (a) Schematic of the light-sheet microscope. (b) Reconstructed helical 3D trajectory of a density matched $50 \mu \mathrm{m}$ sized swimmer at $c_{s}=7.5 \mathrm{wt} \%$. (c) Azimuthal angle $\phi$, height $h$ along helical axis and curvature $r$ vs. time for the trajectory depicted in (b).

isotropic average $\eta_{\text {iso }} \approx \frac{1}{3} \eta_{2}[90]$. In consequence, the Marangoni surface flow will be stronger near the defect and this asymmetry adds a torque to the motion of the droplet and hence, a rotational component.

By tracking $1 \mu \mathrm{m}$ sized colloidal tracers in the continuous phase close to the interface $(\approx 4 \mu \mathrm{m})$, we indeed find that the surface velocity increases near the defect location. The defect vector always points to the convex side of the curved trajectory, followed by a faster surface flow (Fig. 5.26).

We have plotted the average angle $\Theta_{s}$ enclosed by the defect and the velocity vector versus the average droplet speed $v$ (see Fig. 5.26b). $\Theta_{s}$ increases with $v$ in a nonlinear manner. Qualitatively, we see that the defect will be pulled further towards the droplet equator for increasing $v$. In turn, the surface speed $v_{s}$ increases, such that we can expect a $\Theta_{s} \propto v^{2}$ component for small $\Theta_{s}$. Moreover, the small angle approximation holds true and thus, $v_{s} \propto \sin \theta \approx \theta$. For smaller droplets with diameters of $d=37 \mu \mathrm{m}, \Theta_{s}$ increases less with $v$. Here, the same topological charge of the defect applies to a smaller volume, thus requiring a higher deformation energy.

We further addressed the question of how the droplets propel in an unconfined environment, since rotation can also be caused by interaction with a solid interface, as known from bioflagellates like E. coli [24, 31]. By adding heavy water $\left(\mathrm{D}_{2} \mathrm{O}\right)$ to the surfactant solution, the densities of the aqueous phase and the swimming droplets can be matched. We recorded 3D trajectories with a custom built light-sheet fluorescence microscope in a volume of $3 \times 3 \times 3 \mathrm{~mm}^{3}$, as shown in Fig. 5.27. We found helices with well defined pitch $p$, azimuthal angle $\phi$ and curvature $r$ over the persistence length of the overall trajectory (cf. example in Fig. 5.27p, c with 
$r=55 \pm 4 \mu \mathrm{m}, p=100 \pm 20 \mu \mathrm{m}, v=17.4 \pm 3 \mu \mathrm{m} / \mathrm{s})$. The experimental conditions were kept similar to the quasi-2D study, except for the addition of $\mathrm{D}_{2} \mathrm{O}$ to the surfactant solution and doping the 5CB droplets with the fluorescent dye NileRed.

The instability that results in helical swimming cannot be caused solely by a static deflection of the defect vector. If the force on the defect is purely polar without any azimuthal component, as assumed for our quasi-2D system, the trajectory should be circular and confined to the plane spanned by the velocity and the defect vector (see Fig. 5.28, (right, 1)) [42]. However, if a swimmer finalizes one orbit to meet its trajectory, it will encounter a repulsive chemotactic gradient caused by its own trail of filled surfactant micelles. In turn, this gradient leads to an out-of-plane force component. Effectively, the swimmer is pushed into a direction perpendicular to the plane of rotation, leading to a stable helical trajectory within the time frame of persistent swimming (Fig. 5.28, (right, 3)).

A constant external force, e.g. gravity, would also lead to helical trajectories but with the helical axis aligning with the force vector. Trajectories from an experiment with several droplets are shown in the left panel of Fig. 5.28. The helix axes show no global directional bias, especially not in the direction of gravity with $\vec{g} \| \vec{z}$. From the eleven recorded trajectories, five helices are right handed (blue) and six are left handed (red). This equidistribution confirms that there is no global chirality in the system, as expected from the symmetry of the underlying flow instability. The autochemotactic repulsion furthermore provides a good

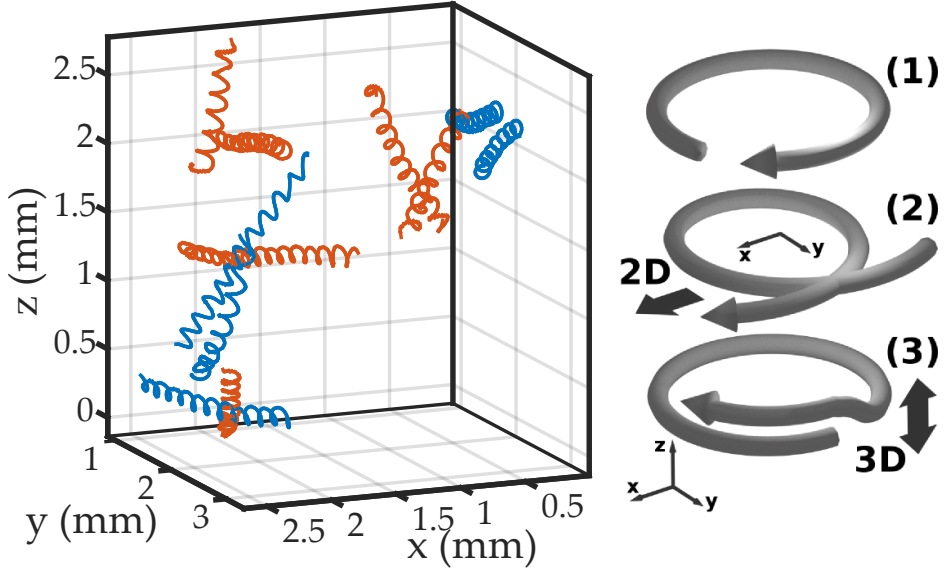

Fig. 5.28: Left: Trajectories of 11 droplets in a buoyancymatched aqueous TTAB solution of $c_{s}=5 \mathrm{wt} \%$ at room temperature recorded over a time span of 5 minutes. The trajectories show no global bias of orientation or chirality (in blue 5 right handed and in red 6 left handed trajectories). Right: Conceptual sketch of trail-avoidance strategies. (1) The initial undisturbed orbit. In 2D (2), the resulting autochemotactic force (black arrow) is in the $x y$-plane and leads to a trajectory crossing. In 3D (3), the force vector points parallel or anti-parallel to the orbit normal. explanation for the behaviour in the 2D environment (Fig. 5.28, (right, 2)). Here, the droplets do not move along a circular trajectory but show a net propagation that points away from regions containing filled micelles (cf. Fig. 5.26a).

To summarize, curling or helical swimming in active droplets results from a spontaneous symmetry breaking with two-steps. The first step results in self-propelled motion. The second step, which is only present when the droplets are in the nematic state, adds a rotational 
component to the active motion. Thus, although the droplets are radially symmetric at rest, they show curling motion in 2D and helical swimming in 3D, purely driven by spontaneously emerging, dynamic flow instabilities and autochemotactic effects.

Hitherto, the behaviour of droplets in rather low surfactant concentrations was observed. If we increase the surfactant concentration $c_{s}$ above $\approx 17.5 \mathrm{wt} \% \mathrm{TTAB}$, the autochemotactic effects are strong enough to fully repel the droplets from their own trajectories, resulting in strictly self-avoiding trajectories even in a confined 2D environment. This switching of the characteristic motion by varying the surfactant concentration offers another perspective to gain insights into the sensitivity of LC droplets to surfactant gradients. Furthermore, this extends the study discussed in Sec.5.1.4.

In Fig. 5.29, 2D trajectories at $c_{s}=20 \mathrm{wt} \%$ TTAB are shown. Instead of curling, meandering motion emerges. Thus, the autochemotactic repulsion results in a characteristic sharp turn when a droplet approaches its own trajectory. The main differences between the systems of curling and meandering are the number of micelles in the bulk solution, the solubilization rate and the velocity of the droplets.

The quantities of interest emerging from the measurements are the mean velocity $v_{\text {mean }}$, the period length $t_{p}$ and the travelled circumference $d r$. These values were retrieved at three different temperatures for $c_{s}=7.5 \mathrm{wt} \%$ TTAB and for $20 \mathrm{wt} \%$ TTAB at $\mathrm{T}=22^{\circ} \mathrm{C}$.

The resultant data sets are depicted in Fig. 5.30a. Here, circles indicate experiments at $7.5 \mathrm{wt} \%$ with colour coded temperature increase and diamonds show data at $20 \mathrm{wt} \%$ TTAB. The right column in (a) exemplarily depicts single events investigated, either for the curling or for the meandering case. For curling trajectories of droplets at different temperatures, the circumference of one curl is rather constant between approximately 200 and $270 \mu \mathrm{m}$. Accordingly, the pe-

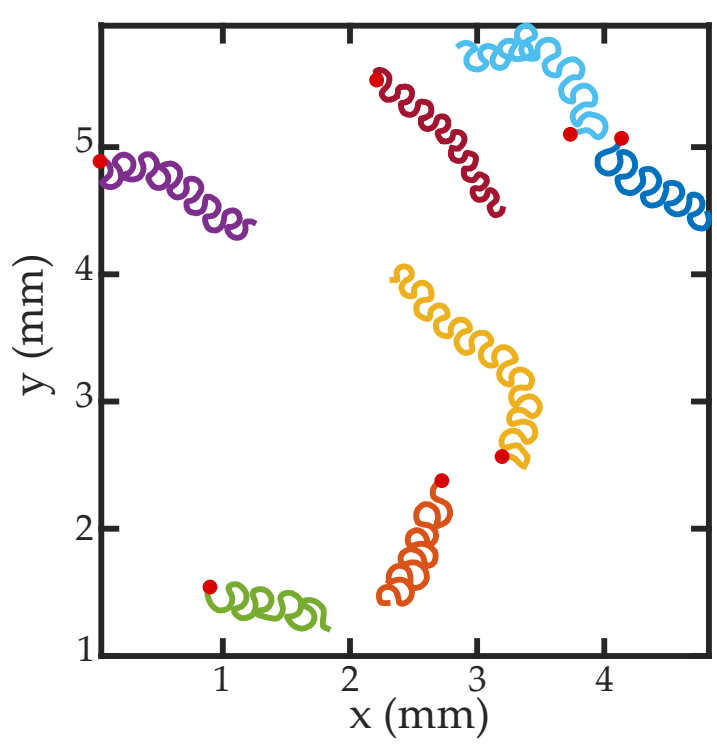

Fig. 5.29: Meandering 2D trajectories of nematic droplets at elevated surfactant concentration $\left(c_{s}=20 \mathrm{wt} \%\right.$ TTAB). Red dots emphasize the starting point of the trajectory. Sharp turns due to autochemotactic repulsion are visible when droplets approach their own trail. riod time decreases, implying temperature dependent velocities (cf. Fig. 5.4). However, crossing the path is still possible and the negative autochemotaxis stays below a threshold value. In the meandering case, the rotation time is longer than for the depicted curling case. Despite 

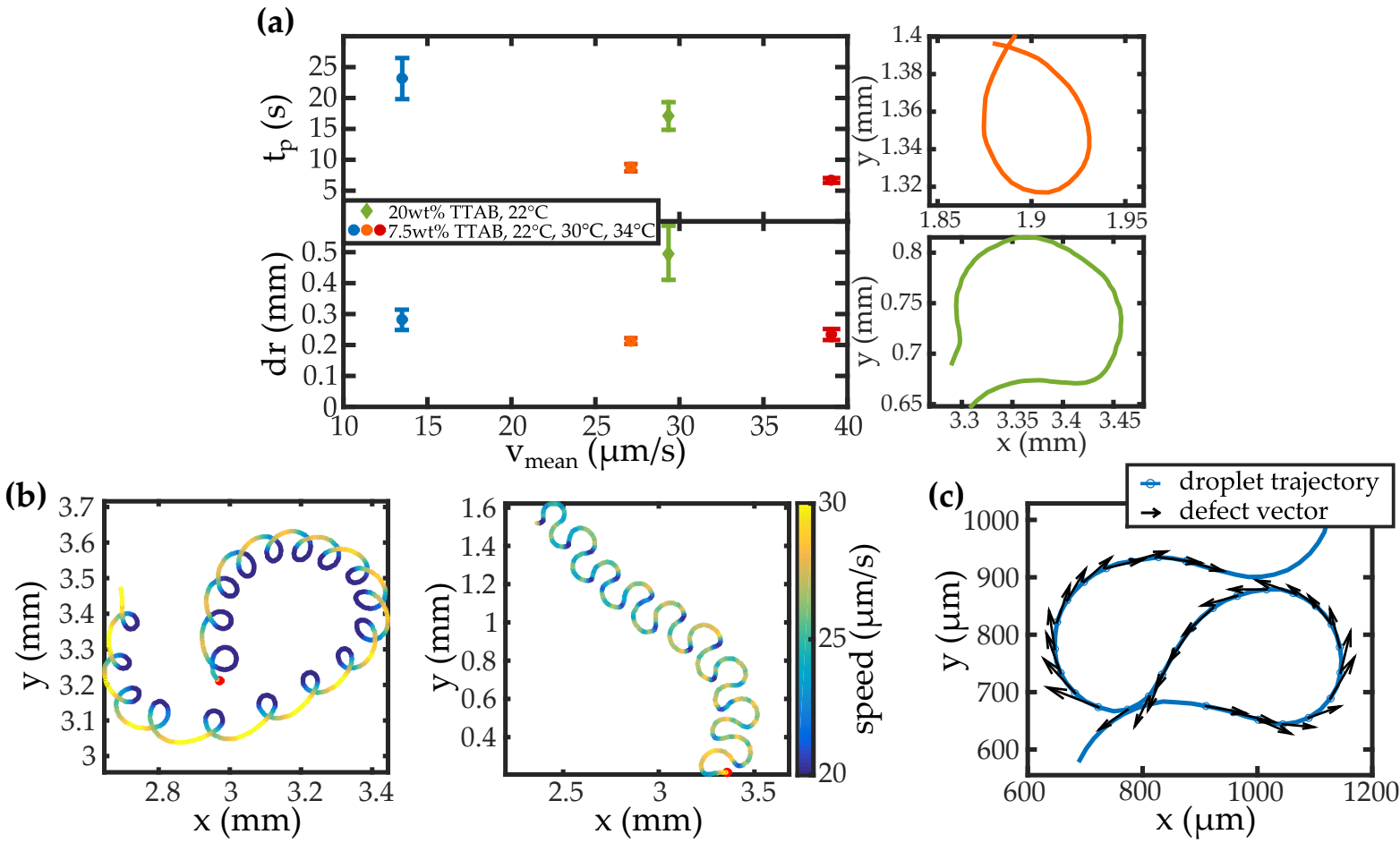

Fig. 5.30: (a) (left, top) Period time $t_{p}$ and (left, bottom) circumference $d r$ of a single rotation vs. the velocity for all discussed cases. Circles show data for $c_{S}=7.5 \mathrm{wt} \%$ at temperatures of 22,30 and $34^{\circ} \mathrm{C}$ (colour code) and green diamonds show data for $c_{S}=20 \mathrm{wt} \%$ at $22^{\circ} \mathrm{C}$. (right) For each case, one example event of curling (top, orange) and meandering (bottom, green) is depicted. The colours are in accordance with the left hand graph. (b) Two example trajectories for each case, colour coded by local velocity. Interestingly, the velocity in the curling case deviates strongly between the curl parts and the straight parts in the trajectory, while for the meandering case, differences are only visible when the droplet approaches its own trajectory. (c) Depiction of the defect orientation (black arrows) along a meandering trajectory.

the increased rotation period and circumference, it is impossible for the droplets to cross their own trajectory.

In the following, we will compare two settings for curling and meandering propulsion with similar velocities $\left(v_{\text {mean }} \approx 28 \mu \mathrm{m} / \mathrm{s}\right)$ and initial droplet diameters $(d=50 \mu \mathrm{m})$ and discuss the implications on the characteristic motion. We focus the discussion on the diffusion characteristics of the partaking species. The respective settings chosen are, first, droplets at $30^{\circ} \mathrm{C}$ in $7.5 \mathrm{wt} \%$ and, second, droplets at room temperature in $20 \mathrm{wt} \%$ TTAB.

Here, the diffusion constants of the micelles change as they increase in size (see Fig. 3.1). Also the diffusivity of the single LC molecules will be altered due to a change in bulk viscosity of the continuous fluid. Both parameter changes depend on the surfactant concentration.

For an assumption of the micelle diffusion constants, the Perrin equation [154] modelling friction factors for spheroids is applied with the data for the micelle geometries from Fig. 3.1 
and the following approximations for the bulk viscosities. We consider values of $\eta(7.5 \mathrm{wt} \%$, $\left.30^{\circ} \mathrm{C}\right)=1 \mathrm{mPa} \cdot \mathrm{s}$ [196] and $\eta\left(20 \mathrm{wt} \%, 20^{\circ} \mathrm{C}\right)=10 \mathrm{mPa} \cdot \mathrm{s}$ [98]. The resulting diffusion constants show a roughly 25 times higher value in the curling case than for the meandering case. This points to the fact that, even with the longer period times in the latter case, it is not possible to decrease the local filled micelle concentration by diffusion below a threshold value. Thus, negative autochemotaxis prevents the droplets to cross their own trails, resulting in the meandering motion.

Furthermore, Fig. 5.30 p depicts one trajectory for each case, both colour coded by velocity. Interestingly, in the curling case, the droplet velocity along the curls is lower than along the straight parts of the trajectory. This might be explained by two reasons. First, an autochemotactic repulsion accelerates the droplets away from the point of crossing, while the approach of a passed region decelerates the droplet (cf. Fig. 5.3b). Or second, a minor movement along the $z$-axis along the curls to avoid the beforehand passed region. The latter reason suggests a confinement effect influencing the appearing characteristic motion. This question can be resolved by further experiments with varying reservoir heights. In the meandering case, the velocity drops only substantially when it approaches its preceding trajectory and is autochemotacticly decelerated.

Hitherto, the meandering behaviour has not been observed in three dimensions and with buoyancy-matched bulk fluid. This suggests that the displacement of the defect into the polar direction is easier than displacing the defect across the droplet apex in the azimuthal plane. As can be seen in the curling case (see Fig. 5.26), the angle between the propulsion direction and the defect position stays rather constant. In the meandering case, the defect has to oscillate across the droplet apex (see Fig. 5.30k). Such a distortion of the elastic nematic field needs much more energy and is therefore unfavourable.

\subsection{Dilute systems in three dimensions}

In the previous chapter, we have already shown results from density matched three-dimensional measurements obtained via light-sheet microscopy. In this section, we will focus in more detail on the change of the relevant quantities, in particular, the pitch $p$, the helix radius $r$ and the period length $T$ in dependence of the surfactant concentration $c_{s}$.

Fig. 5.31 depicts trajectories of $50 \mu \mathrm{m}$ sized droplets at varying surfactant concentrations between 5 and $25 \mathrm{wt} \%$ that have been recorded over $120 \mathrm{~s}$. For low surfactant concentrations, the helical movement is clearly visible. With increasing surfactant concentration, the trajectories get more and more erratic until, starting at $c_{s}=17.5 \mathrm{wt} \%$, no curling is observed any more. The latter behaviour rather indicates active Brownian motion. When analysing the tra- 
jectories with respect to their mean velocity, the values start from $14 \mu \mathrm{m} / \mathrm{s}$ at $5 \mathrm{wt} \%$, increase up to $24 \mu \mathrm{m} / \mathrm{s}$ for a concentration of $15 \mathrm{wt} \%$ and subsequently decrease drastically down to $9.4 \mu \mathrm{m} / \mathrm{s}$ for $c_{s}=25 \mathrm{wt} \%$. The larger decrease in velocity as compared to the one and two dimensionally confined data (cf. Figs. $5.2 \mathrm{a}$ and $5.16 \mathrm{p}$ ) is possibly an artefact due to the low effective frame rate of the light-sheet microscope $(<2 \mathrm{fps})$. This effectively smooths out the erratic movement of the droplets.

(a)

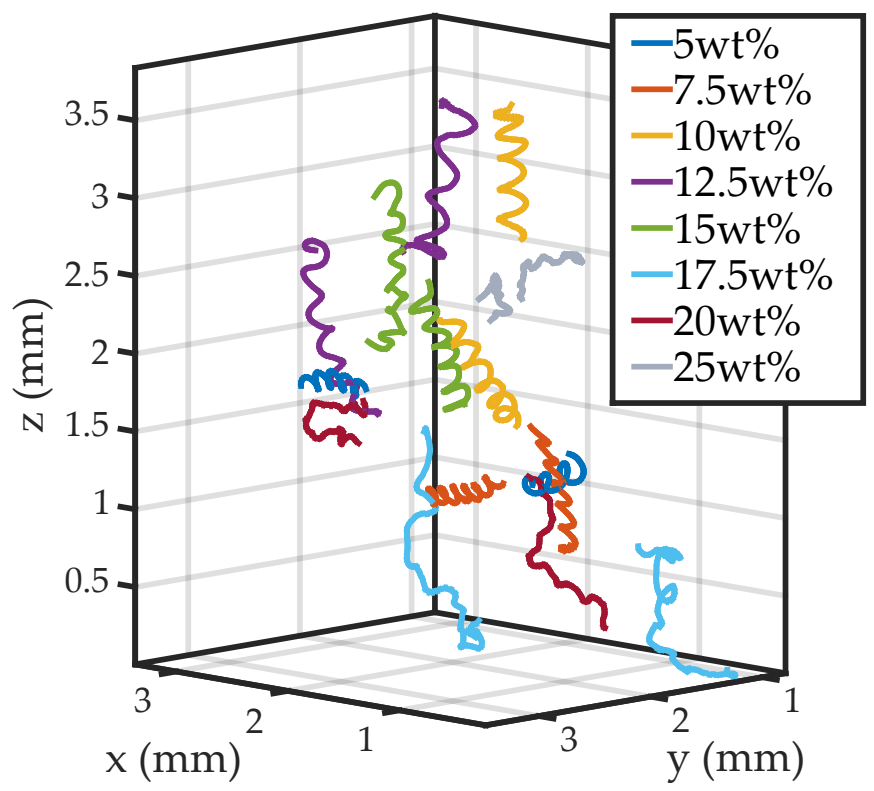

(b)

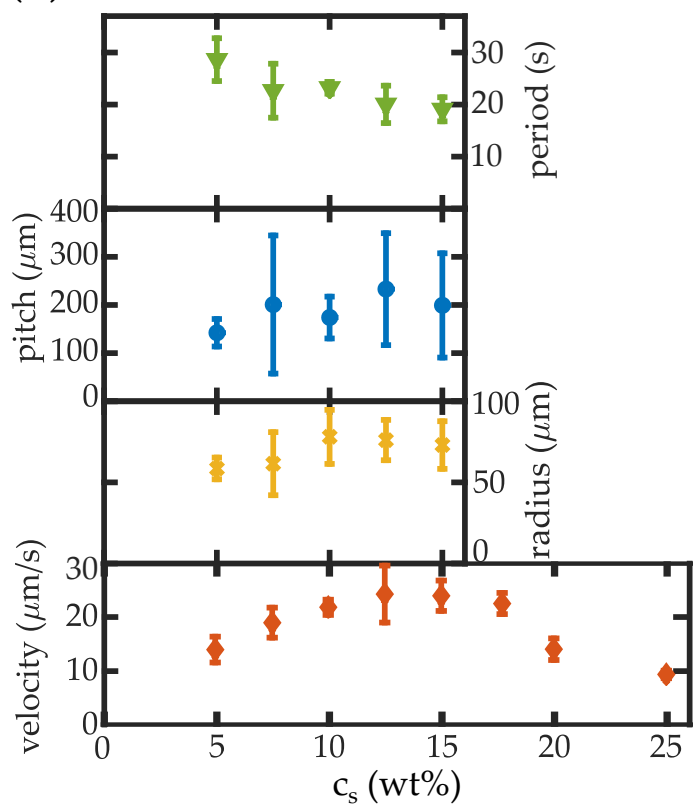

Fig. 5.31: (a) Trajectories of droplets for different surfactant concentrations, recorded over a time span of $120 \mathrm{~s}$. A change in the qualitative behaviour with increasing surfactant concentration is visible $\left(c_{s}>\right.$ $15 \mathrm{wt} \%$ ). (b) Extracted quantities from the trajectories. The velocity is obtained for the whole range of used surfactant concentrations $(5-25 \mathrm{wt} \%)$, while the pitch, period and radius are extracted only for cases where helical motion is observed.

In the case of low surfactant concentrations ( $5 \mathrm{wt} \%<c_{s}<15 \mathrm{wt} \%$ ), helical motion is present and thus, values for the pitch, helical radius and period length can be extracted and are depicted in Fig. 5.31?

The pitch $p$ is by far the most strongly fluctuating quantity, since the sensitivity to autochemotaxis is the main driving force for the pitch and a perfect density match is necessary for robust measurements. Following this reasoning, one would expect an increase in pitch with increasing surfactant concentration and velocity due to stronger autochemotactic interactions. This is consistent with the data shown in the second panel of Fig. $5.31 \mathrm{p}$.

The other quantities exhibit smaller standard deviations. Moreover, they show reasonable agreement with the expectations gained from the measurements mentioned in Sec. 5.2.5 and highlighted in Fig. 5.30. The radius and, likewise, the circumference of one rotation is not 
changing strongly with increasing velocity, only the period $T$ decreases due to the increasing velocity. Even though the droplets are confined differently (2D vs. 3D), the mean helix radius $\bar{r}_{h}$ in $3 \mathrm{D}(\approx 60 \mu \mathrm{m})$ complies with the mean curl radius $\bar{r}_{c}(\approx 45 \mu \mathrm{m})$. The latter is calculated from the circumference in $2 \mathrm{D}$ with corresponding data depicted in $5.30 \mathrm{a}$ and $5.31 \mathrm{~b}$. This suggests the same physical driving mechanism for both propulsion modes.

With the experimental values, we are able to calculate a viscous torque $M_{\text {visc }}$ using Faxén's law [197] with the assumptions of no background flow and no interactions with other swimmers. The equation is given by

$$
M_{\text {visc }}=8 \pi \eta R^{3} \omega=8 \pi \eta R^{3} \frac{2 \pi}{T}
$$

We calculate $M_{\text {visc }}$ for $c_{s}=7.5 \mathrm{wt} \%$ at ambient temperatures and take the experimental parameters into account. We estimate $\eta\left(22^{\circ}\right)=$ $1.2 \mathrm{mPa} \cdot \mathrm{s}$ [98] and $R=25 \mu \mathrm{m}$. Thus, the viscous torque results to $M_{\text {visc }} \approx 140 \mathrm{pN} \cdot \mu \mathrm{m}$. Furthermore, we split the velocity into one component for the $x y$-plane and one for the $z$-direction. One readily obtains that the second symmetry breaking and generated torque forces the droplet onto a circle with velocity $v_{x y}=17.1 \mu \mathrm{m} / \mathrm{s}$. The autochemotaxis leads to a shift in $z$ with velocity $v_{z}=8.9 \mu \mathrm{m} / \mathrm{s}$, resulting in helical trajectories. This is in good

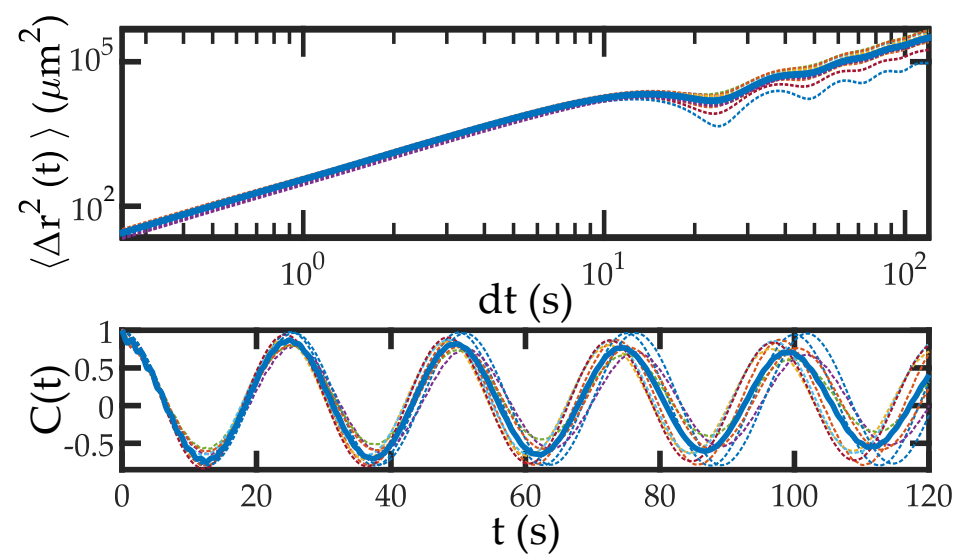

Fig. 5.32: Analysis of helical trajectories depicted in Fig. 5.28(left). The top graph displays the MSD, with the characteristic dip at $\approx 22 \mathrm{~s}$. Subsequently, an oscillatory behaviour in the MSD can be observed. (bottom) Velocity autocorrelation of the trajectories. Strong oscillations are visible with a periodicity of $\approx 24 \mathrm{~s}$, corresponding to the dip in the MSD. In both cases, dash-dotted lines show data of single experiments, while the thick blue line is the average of the data set. agreement with the proposed mechanism in Fig. 5.28(right).

For the set of experimentally found trajectories shown in Fig. 5.28 (left), the mean squared displacement and the autocorrelation function are retrieved (see Fig. 5.32). The surfactant concentration is $c_{s}=5 \mathrm{wt} \%$ in this case and the droplet diameter amounts to $50 \mu \mathrm{m}$.

The top graph depicts the MSD. For short times, ballistic motion results in the $t^{2}$ course of the MSD. This regime ends with a characteristic dip due to the helical behaviour at $\approx 22 \mathrm{~s}$. Subsequently, as a further consequence of the helices influencing the MSD, oscillatory behaviour emerges on long timescales. As discussed (cf. Fig. 5.28 (left)), due to the autochemotaxis and a thus resultant propagation into the $z$-direction, the MSD still exhibits a net slope close to two. 
In the case of the autocorrelation $C(t)$ (Fig. 5.32, bottom), strong oscillations, similar to the $2 \mathrm{D}$ behaviour (see Fig. $5.24 \mathrm{p}$ ), are visible. Since these exhibit a periodicity of $\approx 24 \mathrm{~s}$, they are in accordance to the dip in the MSD. The stable oscillatory amplitude in $C(t)$ is another indicator for the persistent helical swimming of the LC droplets. Finally, both values confirm the measured period length that is shown in Fig. $5.31 \mathrm{p}$.

So far, a behaviour that is comparable to our observations has only been described in simulations on positive autochemotactic swimmers with strong coupling to the self-generated trails [164, 166]. However, the effect is less pronounced in the LC system, since a net propulsion that points away from the starting position is still maintained.

It is interesting to note that the introduction of a homogeneous magnetic field on the order of $100 \mathrm{mT}$ results in ceasing of the helical motion. In Fig. 5.33, trajectories with the same experimental settings as in Fig. 5.31 but with an additional magnetic field are shown. In this case, the blue arrows depict the direction of the magnetic field. No helical motion is observed, comparable to the

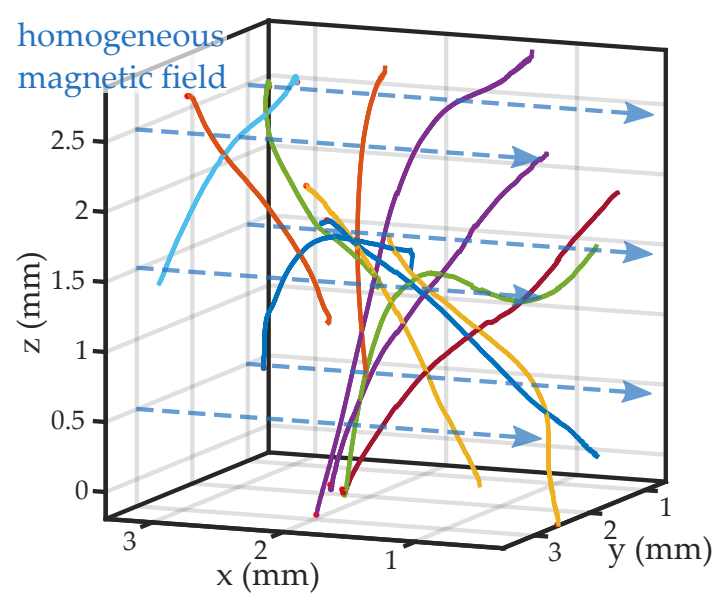

Fig. 5.33: Trajectories in LSM with applied magnetic field. The experimental settings are similar to Fig. 5.31a, with an additional magnetic field depicted by the blue arrows. Helical motion ceases completely and persistent swimming emerges. case at elevated temperatures in the isotropic phase (see Fig. 5.25). However, instead of decreasing the ordering of the molecules, the magnetic field fixes the director structure against the fluctuations due to the internal convection. Thus, no torque operates on the droplets and the propulsion behaviour changes to ballistic motion.

\subsection{Dense systems in two and three dimensions}

Up to now, we showed that the swimmer behaviour can be controlled by system parameters like surfactant concentration or temperature, leading to changes in the propulsion characteristics and speed. However, the droplets were investigated mostly in a confined geometry and at low-number densities. To mimic dense biological systems in a more realistic environment, the number density and dimensionality has to be increased. In this respect, we will investigate suspensions of a high number of droplet swimmers in terms of varied confinement and changing buoyancy settings. 


\subsubsection{Influence of the dimensionality on the collective behaviour ${ }^{2}$}

In Sec. 2.1.1. large scale dynamics of bacterial colonies or of plankton distributions in the oceans have been discussed. Both cases are difficult to trace with simulations and experimental studies on artificial systems are highly desired. They are of even higher interest, if these artificial systems are sensitive to the interplay between self-propulsion, buoyancy and advection. In this respect, our LC system shows promising features. In particular, our system has a high symmetry, scalability, long lifetimes and the option to tune speed, dimensionality and even features of motion characteristics.

As shown in the following, we encountered a novel kind of self-assembled clustering that is driven by convection. This clustering cannot be understood in a framework lacking either hydrodynamics or a full 3D description, in particular when high numbers of droplet swimmers are observed under varying dimensional confinement and buoyancy settings.

Therefore, the experimental settings were adapted to address these specific issues. A typical experiment consists of a large number of droplets ( $>1000)$ with initial diameter of $a=45 \mu \mathrm{m}$ in cylindrical PDMS reservoirs with a diameter $D$ of $6 \mathrm{~mm}$ and heights $H$ varying between $0.3 \mathrm{~mm}$ and $5 \mathrm{~mm}$ (see Fig. 5.34). Surfactant concentrations $c_{s}$ range between $7.5 \mathrm{wt} \%$ and $17.5 \mathrm{wt} \%$. We observe the in-plane droplet motion with bright-field microscopy with a $2 \times$ objective lens and record data at frame rates of $4 \mathrm{fps}$. For height measurements of clusters, we use a calibrated z-stage and an objective lens of $40 \times$ magnification. The latter microscopy settings are also used to map fluid motion in the aqueous phase by particle tracking or particle image velocimetry (PTV/PIV). Here, $2 \mu \mathrm{m}$ sized latex colloids serve as tracer particles. By admixture of heavy water $\mathrm{D}_{2} \mathrm{O}$, we generate different buoyancy settings depending on the fraction of $\mathrm{H}_{2} \mathrm{O} / \mathrm{D}_{2} \mathrm{O}$ in the surfactant solution (see Sec.4.4).

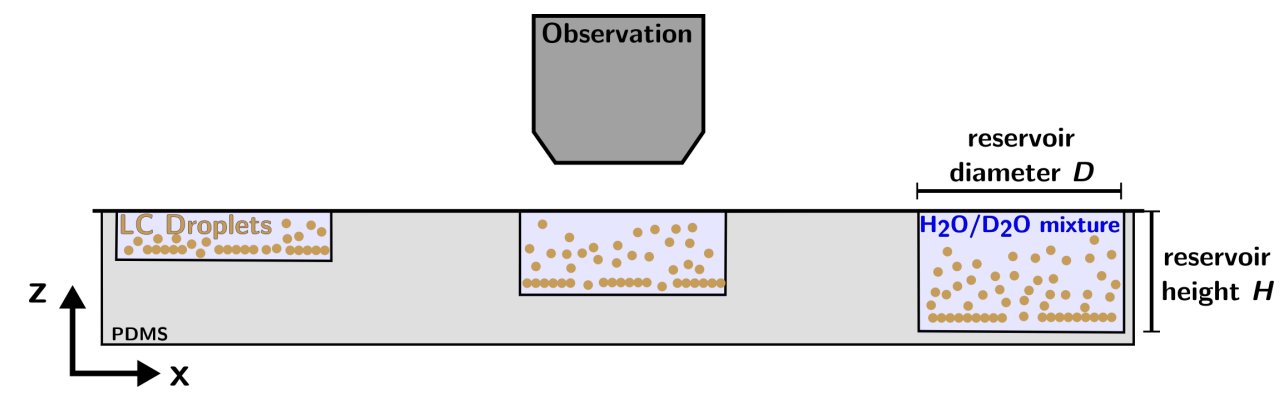

Fig. 5.34: Schematic illustration of the experimental chamber. Reservoirs of different height $H$ are made by mould casting PDMS. The surfactant solution mixed with droplets is filled into the reservoirs. The reservoirs are closed by a cover slip to prevent evaporation. Depending on the $\mathrm{H}_{2} \mathrm{O} / \mathrm{D}_{2} \mathrm{O}$ ratio, clusters will form at either the lower or upper reservoir boundary. The clustering is observed under bright field microscopy.

\footnotetext{
${ }^{2}$ The content of this section is published in: C. Krüger et al. Dimensionality matters in the collective behaviour of active emulsions, The European Physical Journal E, 39(6), 64 [198]
} 
5.4.1.1 Qualitative observations in dense systems We studied dense systems of sedimenting swimmers with initial area densities above $10 \%$ in cylindrical reservoirs of varying height $H$ (Fig. 5.35). We chose the $x y$ plane of observation to align with the reservoir bottom and the $z$-axis to point upwards and thus, against gravity (cf. the coordinate system in Fig. 5.34.

When large numbers of droplets are confined to a quasi-2D layer $(H \approx a)$, the clustering behaviour is qualitatively similar to the low density limit of numerical simulations by Zöttl et al. [199], with only transient clusters (Fig. 5.35, left). Here, the droplets are arrested after headon collisions until rotational diffusion enables them to leave such a transient cluster (cf. also experiments on Janus-particles from Ref. [57]). We were not able to compare our experiments with simulations at higher number densities because the rapid depletion of empty micelles at these number densities will stop droplet motion after less than 5 minutes. Thus, structures cannot fully evolve on the time scale at hand.

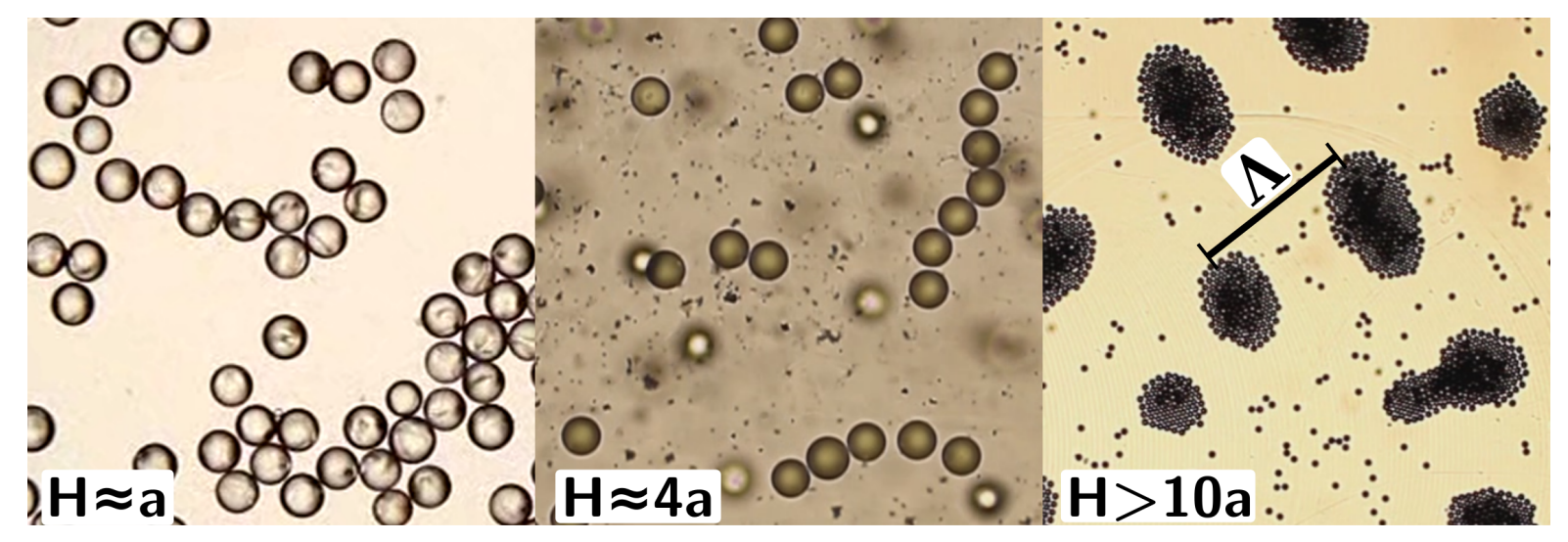

Fig. 5.35: The collective droplet behaviour is controlled by dimensional confinement, i.e. different ratios of reservoir height $H$ and droplet diameter $a$. In a quasi-2D confinement $(H \approx a)$, there are only transient aggregates that develop and break apart dynamically. For intermediate reservoir heights $(H \approx 4 a)$, hydrodynamically stabilized lines are formed perpendicular to the swimming direction. For unconfined reservoirs $(H>10 a)$, stable clusters with the average distance $\Lambda(H)$ assemble over large length scales. The droplet size is $a \approx 45 \mu \mathrm{m}$ in all three images. The field-of-view is $500 \times 500 \mu \mathrm{m}^{2}$ for the left and the middle, and $2.5 \times 2.5 \mathrm{~mm}^{2}$ for the right image.

Long-range hydrodynamic attraction is screened by the $2 \mathrm{D}$ confinement. In particular, the short distance between swimmer and cell boundary sets a cut-off length for the inter-particle interaction [197]. Consequently, if the reservoir height is increased to a few droplet diameters $(H \approx 4 a)$, the droplet behaviour changes (Fig. 5.35. middle). In Fig. 5.13a, the hydrodynamic flow field of the droplets is depicted. The fast fluid flow in the equatorial plane of the droplet acts attractively on other entities. Thus, droplets arrange in stable lines along their equatorial plane, perpendicular to the direction of motion [17, 200, 201] and continue moving collectively. These line arrangements are stable until they collide with single droplets or other lines. 
In a third geometric configuration, extending the reservoir height $H$ to more than 7-10 droplet diameters, we observe the formation of large, stable clusters of hexagonally packed monolayers (Fig. 5.35, right). At higher densities, the clusters are extended into second layer packing.

The time series in Fig. 5.36 depicts the dynamics of cluster assembly over the course of $30 \mathrm{~min}$. A mixture of surfactant solution and individual droplets at the desired number density $n_{d}$ is injected into the reservoir. As the mass density of the swimmers exceeds that of the surfactant solution by $2 \%$, they sediment and accumulate close to the bottom of the reservoir. When the number density of the droplets near the bottom is sufficiently high, they nucleate into flat, hexagonally packed clusters. These clusters are stable for most of the life time of the droplets (neglecting smaller rearrangements at the cluster edges) and coexist with a dilute gas-like phase of freely swimming droplets around them. The clusters hover at a finite height above the reservoir bottom and are rather stationary when compared to the single swimmers. The stable regime ends when the diameter of the droplets shrinks below a critical value during dissolution. Then, clusters start to disintegrate, sometimes buckling in the z-direction, accelerating in oblique directions and collide with the reservoir boundary or disassemble radially (not depicted in Fig. 5.36).

$\mathbf{t}(\min ) \quad 0$

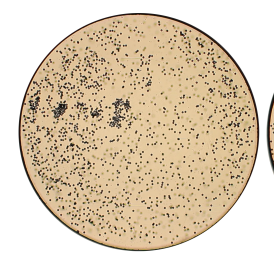

1

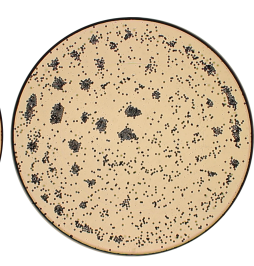

3

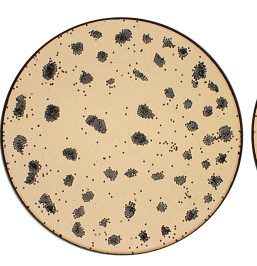

10

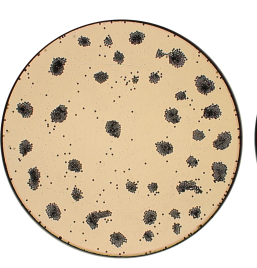

30

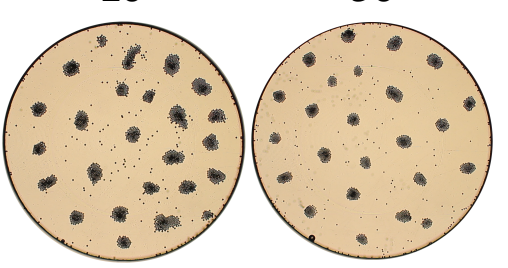

Fig. 5.36: Time lapse of one clustering experiment over the course of $30 \mathrm{~min}$. The height of the reservoir is $2 \mathrm{~mm}$, the reservoir diameter is $6 \mathrm{~mm}$, the initial particle diameter is $45 \mu \mathrm{m}$ and the surfactant concentration $c_{S}$ is $10 \mathrm{wt} \%$ TTAB. Respective times given above.

We will confine our quantitative analysis to the stable regime. Here, cluster mobility is low and rearrangements are infrequent.

5.4.1.2 Morphology of individual clusters To investigate the morphology of individual clusters, we make use of the optical anisotropy of the mesogenic droplet swimmers. As shown in Secs. 5.1.2 and 5.2.5, characteristic topological features can be observed between crossed polarizers. Since the deformation of the director field near the point defect results in a typical birefringence pattern under a polarizing microscope, we can determine the position of the defect and the orientation of the symmetry axis of the swimming droplet.

Fig. 5.37 shows a top view on a cluster between crossed polarizers. The cross-shaped pattern inside each droplet marks the position of the defect and thus, of the leading edge of the 
droplet. In the centre of the cluster, droplets point upwards in $z$. The defect of the droplets on the boundary are tilting inwards and enclose an average angle $\alpha$ of $25 \pm 6^{\circ}$ with the $z$-axis.

As the droplets are oriented upwards, they pump liquid downwards due to their surface flow field. Thereby, they generate a stagnation pressure that equilibrates the cluster at a finite height $h$ above the reservoir bottom $(z=0)$. This mechanism is confirmed by the observation that $h$ increases linearly with the typical swimming velocity $v_{\text {free }}$. This velocity $v_{\text {free }}$ refers to a freely swimming droplet in a surfactant solution of comparable concentration (see Fig. 5.41). From this observation we imply that $h$ increases with the pumping power of the cluster. An outflow of fluid from below the cluster and an influx from above suggest a closed toroidal convection roll as sketched in Fig. 5.37p.
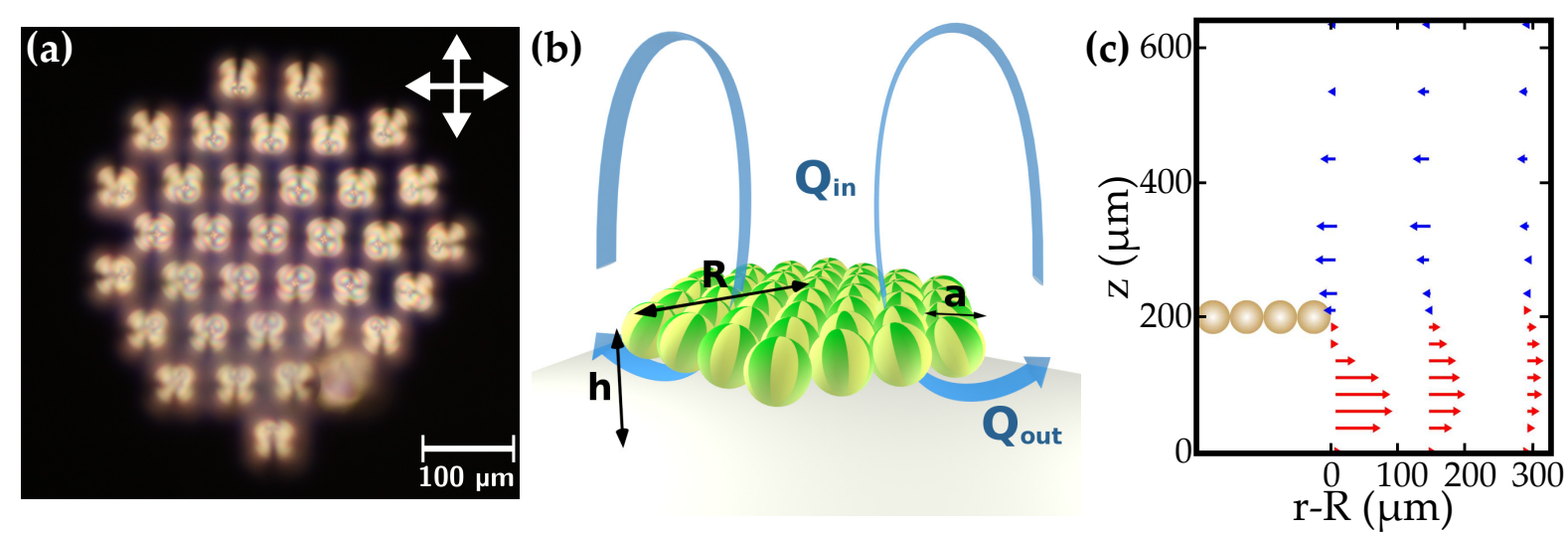

Fig. 5.37: (a) Morphology of a stable cluster observed between crossed polarizers. The cross-shaped pattern inside each droplet marks the position of the defect. Droplets in the centre point upwards and the tilted defect alignment on the boundaries encloses an angle of $\alpha=25 \pm 6^{\circ}$ with the $z$-axis. The white arrows show the orientation of the polarizers. (b) Schematic illustration of a stable cluster consisting of a single discoidal droplet layer with radius $R$ and hovering height $h$. The droplet diameter is $a$ and the stripe pattern symbolizes the defect position and orientation. The volume influx $Q_{i n}$ equals the outward flowing volume $Q_{\text {out }}$, indicating a toroidal convection roll. (c) Flow field around a stable cluster traced with $2 \mu \mathrm{m}$ sized latex particles. The spheres emphasize a cross section of the cluster. The radial flow velocity $v_{r}$ is determined at three different distances to the cluster edge and in various planes possessing different heights $z$ above the bottom of the reservoir $(z=0): v_{r}$ shows a Poisseuillelike profile, directed along $+r$ (away from the cluster centre) below the cluster and along $-r$ (towards the cluster centre) above.

For a quantitative measurement of the structure of the convection roll, we mixed the surfactant solution with $2 \mu \mathrm{m}$ sized latex tracer particles at a density below the single scattering limit and analysed their motion using PTV. In cylindrical coordinates, we consider the centre of a cluster of radius $R$ to be located at $r=0$ and $z=h$. We determined the radial component of the flow velocity, $v_{r}$, at three different distances from the cluster edge by recording the trajectories of the tracer particles. This measurement was repeated in several focal planes in the range between $z=0$ and $z \approx 3 h$. 
The complete data set maps the three dimensional profile for $v_{r}$ (Fig.5.37k). Below the cluster, $v_{r}$ is directed along $+r$, thus pointing away from the cluster centre. It increases in magnitude from the reservoir bottom to a height approximately in the middle between bottom and cluster $(z \approx h / 2)$. Then, $v_{r}$ decreases until the height of the cluster is reached $(z=h)$. Above the cluster, the aqueous phase is pumped inwards. This is indicated by the flow that is directed along $-r$. The speed $v_{r}$ increases up to a height $z \approx 2 h$ above this height it decreases again. Below the cluster, the average absolute value of $v_{r}$ is larger than above the cluster. We were not able to quantitatively map tracer motion in $z$ but observed a flux in the direction of $+z$ for $r>R$ and $-z$ for $r<R$. The flux exceeds the sedimentation speed of the colloids and was qualitatively monitored by watching tracers move through the focal plane at the cluster position and passing through the interstices between the droplets. This completes the picture of the toroidal convection roll as sketched in Fig. 5.37p.

We can now derive a correlation between the cluster radius $R$ and the hovering height $h$. Therefore, we use the simple assumption that the total inward flux through the cluster has to be equal to the outgoing flux below the cluster.

We consider a single-layered discoidal cluster, with its centre located at $r=0$ and $z=h$. Inside the cluster, droplets with radius $\frac{a}{2}$ arrange hexagonally with their leading edge pointing upwards. If a freely floating droplet moves at a speed $v_{i n}$, each droplet within the cluster will displace fluid at a speed $v_{\text {in }}$ times its cross section $\pi\left(\frac{a}{2}\right)^{2}$. A summation over the entire cross section of all droplets yields a cluster area $A_{c l}=\frac{\pi^{2}}{2 \sqrt{3}} R^{2}$. The total inward flux is given by $Q_{i n}=v_{i n} A_{c l}$ and has to be balanced by an outward flux $Q_{o u t}$. The area $A_{f l}$ that has to be passed by the outward flux can be described by the boundary of a cylindrical volume with radius $R$ and height $h$ that is spanned between the cluster and the bottom of the reservoir. Thus, $A_{f l}=2 \pi R h$. With $v_{\text {out }}$ denoting the mean velocity of the outward flow, we have $Q_{\text {out }}=v_{\text {out }} A_{f l}$. The flux balance requires

$$
Q_{\text {in }}=v_{\text {in }} A_{c l} \stackrel{!}{=} v_{o u t} A_{f l}=Q_{o u t}
$$

If we calculate $Q_{\text {out }}$ by integrating the velocity $v_{\text {out }}$ (data shown in Fig. 5.37k) over the respective area, we find $v_{\text {in }} \approx 11 \mu \mathrm{m} / \mathrm{s}$. Note that $v_{\text {out }}$ is measured by projecting a three-dimensional flow into a two-dimensional plane and thus, the component $v_{z}$ is neglected. Under this aspect, the calculated value for $v_{\text {in }}$ corresponds reasonably to the velocity of a single unconfined swimmer with $v_{\text {free }} \approx 19.5 \mu \mathrm{m} / \mathrm{s}$ (see Fig. $5.16 \mathrm{p}$ ).

The swimmers located at the edge of the cluster have to withstand any flow directed along $+r$. Since these swimmers are tilted by an angle $\alpha$, we can assign a radial velocity component $v_{r}=-v_{\text {in }} \sin \alpha$ to them. Assuming that these swimmers experience an outward flow of the surrounding fluid along $+r$ with a mean velocity $v_{\text {out }}$, a simple stability criterion for the cluster 


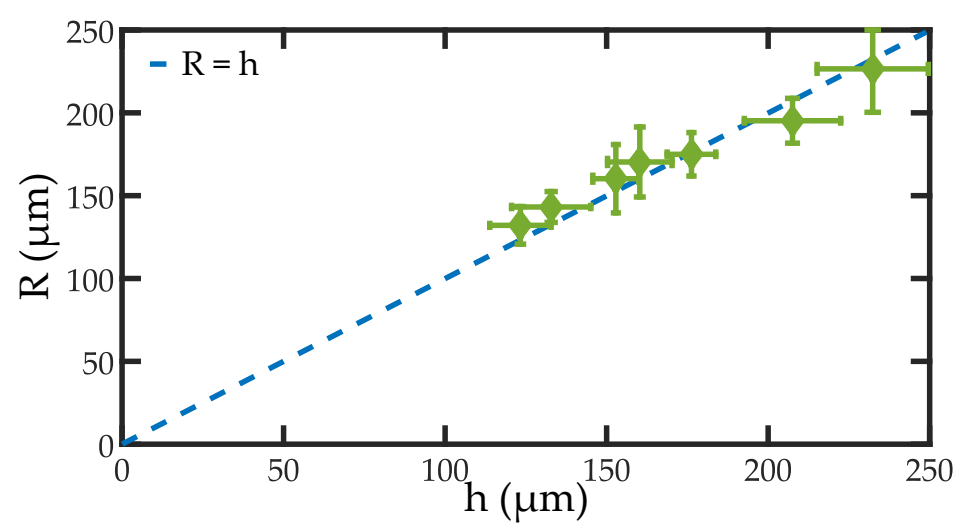

Fig. 5.38: Cluster radius $R$ vs. hovering height $h$. The dashed line assumes a linear relation between $R$ and $h$ with an angle $\alpha$ of $27^{\circ}$ (eq. (5.16)). Chosen surfactant concentrations between 10 and $16 \mathrm{wt} \%$ result in droplet speeds $v_{\text {free }}$ between 19 and $23 \mu \mathrm{m} / \mathrm{s}$.

is $v_{\text {out }} \leq v_{\text {in }} \sin \alpha$. If $v_{\text {out }}$ is larger than this threshold, the swimmers at the cluster boundary would detach from the cluster. Including this criterion in eq. (5.15) and solving for $R$ results in

$$
R=\frac{4 \sqrt{3}}{\pi} h \sin \alpha \approx 2.2 h \sin \alpha .
$$

As shown in Fig. 5.38, we find a linear relation with $R \approx h$ that corresponds to $\alpha=27^{\circ}$ in our experiments. This is consistent with observations from polarizing microscopy (Fig. 5.37 ) that suggest $\alpha=25 \pm 6^{\circ}$. An increasing droplet velocity leads to an increase of $h$ and accordingly to an increase of $R$ (see Fig. 5.38).

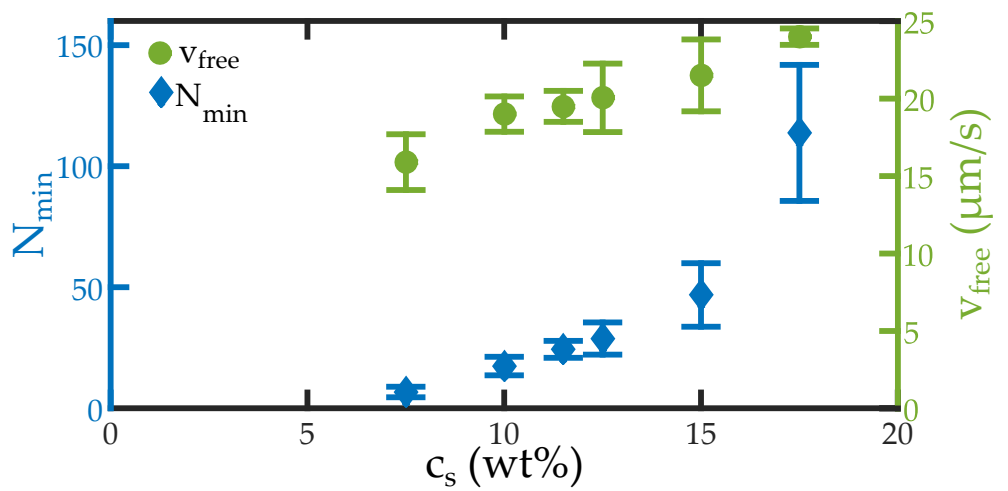

Fig. 5.39: Minimal droplet number for clustering $N_{\min }$ (blue diamonds) and free droplet velocity $v_{\text {free }}$ (green circles) as a function of the surfactant concentration $c_{s}$. The increase of velocity and decrease of reorientation time results in an increase of $N_{\text {min }}$.

The dependence of cluster formation on the droplet velocity and reorientation time in unconfined geometries can further be described by the minimum number of droplets $N_{\text {min }}$ necessary to form a cluster (see Fig. 5.39). For low surfactant concentrations, $N_{\min }$ will be smaller than for higher concentrations. This dependency results from the fact that the assembly of a cluster 
is biased by the droplet speed and especially by its reorientation time. The former quantity increases and the second decreases with increasing surfactant concentration.

For a surfactant concentration of $7.5 \mathrm{wt} \%$, seven droplets are already enough to form a stable cluster, while for a surfactant concentration of $17.5 \mathrm{wt} \%$ more than 100 droplets are necessary to ensure sufficient arresting. The strong increase of $N_{\min }$ from 15 to $17.5 \mathrm{wt} \%$ suggests a threshold concentration beyond which either the pumping action of the droplets will always exceed gravity or they reorient too fast to nucleate clusters and initiate convection. Accordingly, we observe no clustering when a surfactant concentration of $20 \mathrm{wt} \%$ is exceeded.

Hitherto, I have described how a cluster is formed and which mechanism drives the observed convection roll. Succeeding I will discuss two factors influencing the large scale cluster formation. The following experiments demonstrate that the interplay between the negative buoyancy of the droplets and the hydrodynamic flow field generated by the droplets is a key mechanism for this self-assembling behaviour. First, the effect of confining the convection roll by decreasing the height of the reservoir (Sec. 5.4.1.3) and second, the clustering behaviour of droplets in various buoyancy settings (Sec. 5.4.2.1) is examined.

5.4.1.3 Effect of confinement on the clustering behaviour As described in Sec. 5.4.1.1. a certain minimum height $H_{\min }$ of the reservoir is required to observe the formation of the convection-stabilized clusters. The value of $H_{\text {min }}$ depends on the droplet velocity $v_{\text {free }}$. It varies from $H_{\text {min }} \approx 0.25 \mathrm{~mm}$ for $v_{\text {free }}=19 \mu \mathrm{m} / \mathrm{s}$ to $H_{\text {min }} \approx 1.5 \mathrm{~mm}$ for $v_{\text {free }}=24 \mu \mathrm{m} / \mathrm{s}$. The swimmer velocity determines the flow velocity in the convection roll and thus, the spatial extent of the convection roll. The observed dependence of $H_{\min }$ on $v_{\text {free }}$ is a further indication of the importance of convection for the formation of the clusters. Clusters do not form for $H<H_{\min }$ because there is no space for the convection roll to develop.

In a certain range of reservoir heights $H$ above $H_{\text {min }}$, we still observe a pronounced effect of $H$ on the clustering behaviour. For heights $H$ slightly above $H_{\min }$, a convection roll develops but has to be smaller than in the unconfined case $\left(H \gg H_{\text {min }}\right)$, as it is compressed between bottom and ceiling of the reservoir. A direct measure of the lateral size of the convection roll is the average distance $\Lambda$ between neighbouring clusters (see right panel of Fig. 5.35). If we assume a roughly circular cross section of the convection torus, we can expect an asymptotic limit of $\Lambda=2 H$ for shallow reservoirs which is independent of the swimmer speed. The relation is asymptotical because we cannot exclude compression due to the finite lateral confinement.

Varying $H$ stepwise by changing reservoirs, we measured $\Lambda(H)$ for two swimmer systems with roughly equal swimmer numbers (3500 droplets) of initial diameter $a=45 \mu \mathrm{m}$. This results in an area density $n_{d} \approx 20 \%$ and is constant for all used reservoir heights $H$ due to the sedimentation of the droplets into a narrow volume close to the reservoir bottom. We 


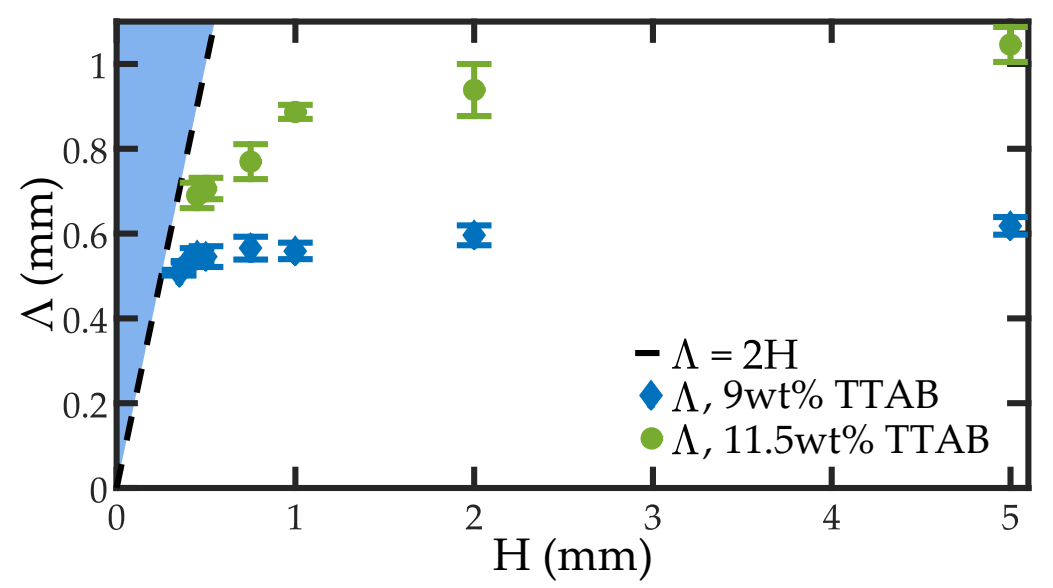

Fig. 5.40: Average cluster distance $\Lambda$ for different reservoir heights $H$. For shallow reservoirs, we observe a linear asymptote $\Lambda=2 H$ (dotted line) beyond which clustering is entirely suppressed (shaded area). For the transition to reservoirs that are unconfined in $z$, a plateauing value is obtained. The two systems consist of $\approx 3500$ droplets $(a=45 \mu \mathrm{m})$ with surfactant concentration $c_{s}=9 \mathrm{wt} \%$ and droplet velocity $v_{\text {free }}=15.5 \mu \mathrm{m} / \mathrm{s}$ (blue diamonds) and $c_{s}=11.5 \mathrm{wt} \%$ and $v_{\text {free }}=19.5 \mu \mathrm{m} / \mathrm{s}$ (green circles).

tune the free swimming speeds $v_{\text {free }}$ of the droplets by changing the TTAB concentration. The first system uses a concentration $c_{S}$ of $11.5 \mathrm{wt} \%$, corresponding to a free swimming speed of $v_{\text {free }}=19.5 \mu \mathrm{m} / \mathrm{s}$ and a minimum for clustering of $H_{\text {min }} \approx 0.45 \mathrm{~mm}$. The corresponding values for the second system are $c_{s}=9 \mathrm{wt} \%, v_{\text {free }}=15.5 \mu \mathrm{m} / \mathrm{s}$ and $H_{\text {min }}=0.35 \mathrm{~mm}$.

We have plotted $\Lambda$ as a function of $H$ in Fig. 5.40. For shallow reservoirs $\left(H_{\text {min }}<H<2 H_{\text {min }}\right)$, $\Lambda=2 H$ is indeed a reasonable asymptote for both data sets. For higher reservoirs, $H$ exceeds the natural extent of the convection roll and $\Lambda(H)$ approaches a constant value. This value increases with the swimming speed, i.e. with the pumping power that drives the convection roll.

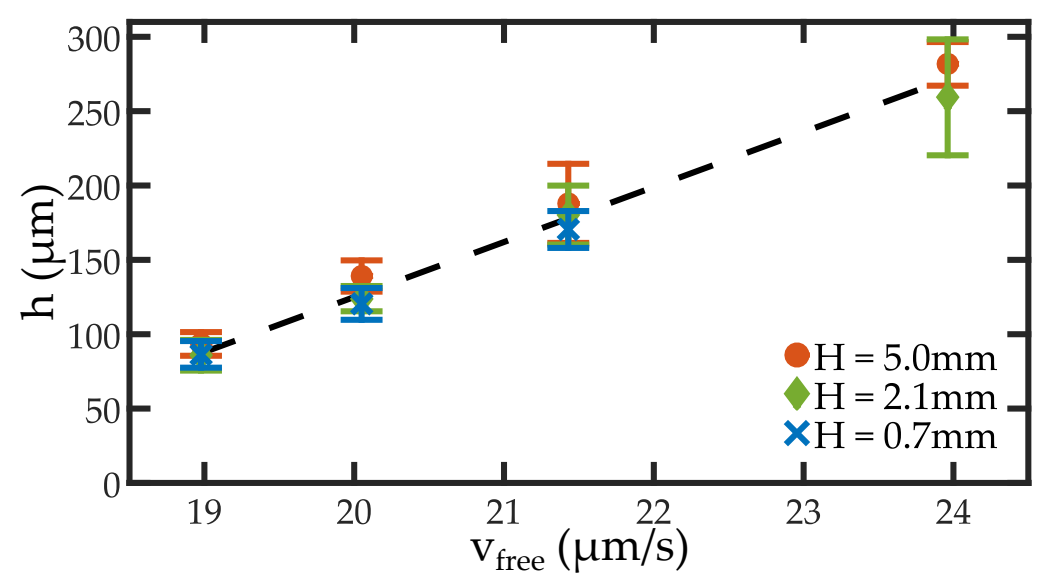

Fig. 5.41: Hovering height $h$ as a function of swimmer velocity $v_{\text {free }}$. Symbols correspond to different reservoir heights $H$. The dashed line marks a linear fit for $h(v)$. 
The reservoir height $H$ has furthermore a subtle influence on the relation between hovering height $h$ and droplet velocity $v_{\text {free }}$. As described in Sec. 5.4.1.2, the clusters hover at a certain distance $h$ above the reservoir bottom as a result of the stagnation pressure generated by the arrested droplets that pump fluid towards the bottom. Accordingly, $h$ increases linearly with the droplet velocity $v_{\text {free }}$ which determines the pumping power of the cluster. If the convection roll is distorted by decreasing $H$, we expect additional pressure contributions from the ceiling of the reservoir and thus, a decrease of $h$. Figure 5.41 depicts $h$ as a function of $v_{\text {free }}$ for three different reservoir heights $H$. The linear dependence of $h$ on $v_{\text {free }}$ is obviously not affected by $H$. However, for a fixed value of $v_{\text {free }}$, we observe a small decrease of $h$ when $H$ is decreased from $5 \mathrm{~mm}$ to $0.7 \mathrm{~mm}$, indicating again the confinement of the convection roll by the reservoir geometry.

\subsubsection{Influence of buoyancy on collective behaviour}

In the previous section, the influence of the confinement on the collective behaviour was investigated. In biological systems often the interplay of buoyancy and the hydrodynamic flow fields of the entities are important to consider. In this respect, the following section investigates the influence of buoyancy adjustment in different geometries.

5.4.2.1 Effect of varying the buoyancy on the clustering behaviour In this section, I report on the effect of varying the buoyancy of the droplet swimmers on their clustering behaviour. The density of $5 \mathrm{CB}, \rho_{5 \mathrm{CB}}$, is slightly larger than the density of the aqueous TTAB solution, $\rho_{\text {aq. }}$. Hence, the droplets sediment at the bottom of the reservoir. We can continuously adjust the density difference $\rho_{5 \mathrm{CB}}-\rho_{\text {aq }}$ without changing the propulsion characteristics of our system by preparing TTAB solutions with varying $\mathrm{H}_{2} \mathrm{O} / \mathrm{D}_{2} \mathrm{O}$ ratio and constant surfactant concentration $c_{s}$. If we approach buoyancy matching while the swimmer velocity is kept constant, swimmers can easily escape the region where a cluster starts forming and the cluster-stabilizing convection cannot develop. Clustering is suppressed when the stagnation pressure below the cluster exceeds gravity.

We added a fixed quantity of $2000-2500$ droplets $\left(n_{d} \approx 12.5 \%\right)$ to the aqueous phase and checked for cluster formation during a time interval of $20 \mathrm{~min}$. Figure $5.42 \mathrm{p}$ depicts a sequence of images, recorded $20 \mathrm{~min}$ after the start of the experiment, for $c_{s}=10 \mathrm{wt} \%$ TTAB and decreasing values of the relative buoyancy mismatch $\Delta \rho$. The latter is given by

$$
\Delta \rho=\frac{\rho_{5 \mathrm{CB}}-\rho_{\mathrm{aq}}}{\left(\rho_{5 \mathrm{CB}}+\rho_{\mathrm{aq}}\right) / 2} .
$$


(a)

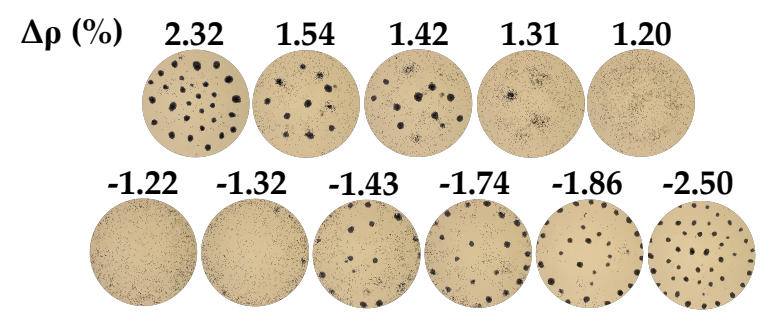

(b)

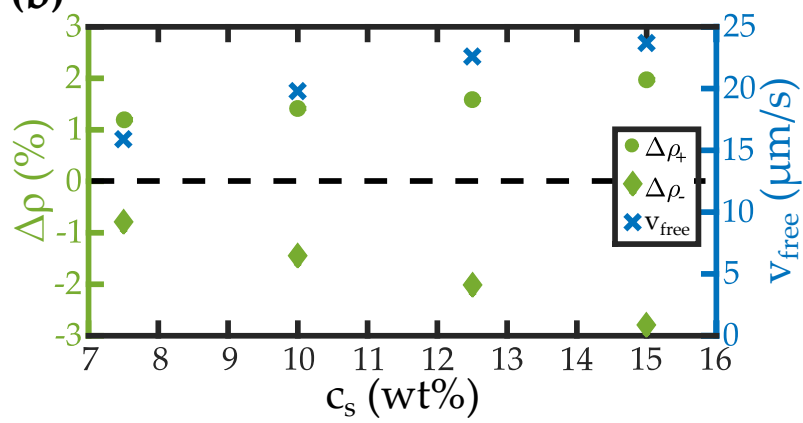

Fig. 5.42: (a) Buoyancy adjustment and cluster formation in a sample with $c_{s}=10 \mathrm{wt} \%$. At a critical density difference $\Delta \rho_{+}=1.31 \%$, the formation of clusters ceases and appears again for negative density differences beyond a threshold of $\Delta \rho_{-}=-1.43 \%$. The reservoir height is $2 \mathrm{~mm}$ and the initial droplet diameter is $45 \mu \mathrm{m}$. Images are taken $20 \mathrm{~min}$ after injecting the droplets. (b) Values of the clustering thresholds $\Delta \rho_{+}$(circles) and $\Delta \rho_{-}$(diamonds) for different surfactant concentrations $c_{s}$. Corresponding droplet velocities $v_{\text {free }}$ are marked by crosses.

We denote the threshold values in relative buoyancy mismatch for clustering with $\Delta \rho_{+}$and $\Delta \rho_{-}$. For the depicted case in Fig. 5.42 , no clustering is observed for $\Delta \rho<\Delta \rho_{+}=1.42 \%$, while clusters reappear for $\Delta \rho<\Delta \rho_{-}=-1.43 \%$. As the swimmers float in this case, the clusters for $\Delta \rho<0$ form at the ceiling of the reservoir.

Figure $5.42 \mathrm{p}$ summarizes the two threshold values for clustering, $\Delta \rho_{+}$for sedimenting and $\Delta \rho_{-}$for floating droplets, for different values of $c_{s}$. The absolute values of $\Delta \rho_{+}$and $\Delta \rho_{-}$ are roughly equal for a given surfactant concentration or droplet velocity. This indicates that clusters consisting of floating droplets are based on the same convection-based mechanism as the clusters of sedimenting droplets. The slight differences in slope between the two cases (see Fig. 5.42 p) might result from the different materials forming the ceiling (glass) and the bottom (PDMS) of the reservoir.

Following the above discussion, a critical ratio of the sedimentation speed $v_{\text {sed }}$ and the velocity of the droplets $v_{\text {free }}$ for clustering is implied. Calculating the ratio $v_{\text {free }} / v_{\text {sed }}$ yields values of $\approx 1.7$. This means, that clustering is suppressed if the propulsion speed exceeds the sedimentation speed by roughly a factor of two. This is intriguing, since a ratio of one already implies equal amounts. Thus, besides gravity, also the interaction of the droplets with each other has to be considered, resulting in the higher escape velocities necessary to suppress cluster formation.

Furthermore, the findings from the preceding sections and their implications are emphasized in a demonstration experiment. To show that the swimmers "sense" the geometry of the accessible space, even without lateral confinement, we provide a geometry where swimmers float in a high $\mathrm{D}_{2} \mathrm{O}$ fraction of $\Delta \rho=-4.35 \%$ at the ceiling of a two-step geometry. The lower part of the latter contains circular reservoirs with $H$ varying between 0.5 and $5 \mathrm{~mm}$ and the upper 


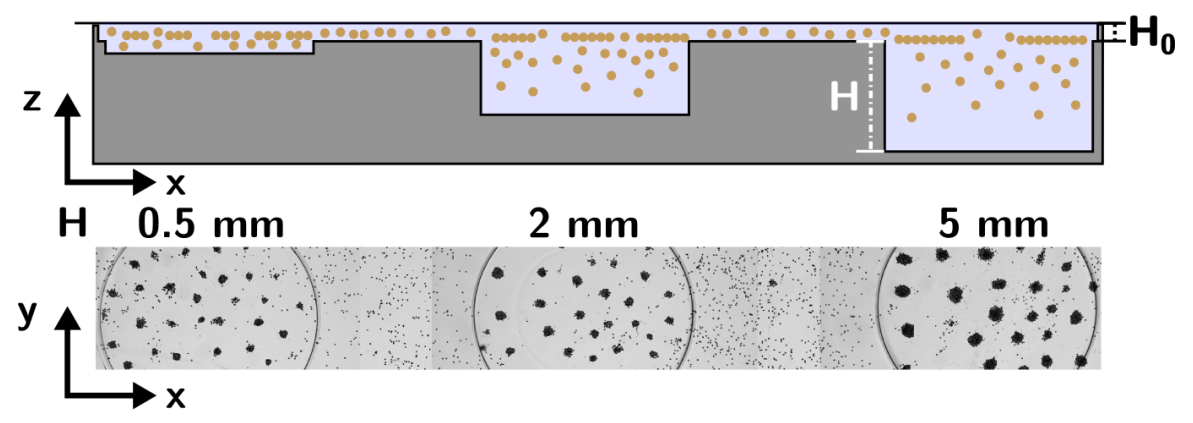

Fig. 5.43: Pattern formation influenced by dimensionality in a two-layer reservoir geometry. The top layer is $H_{0}=300 \mu \mathrm{m}$ high and thus, below the clustering limit. The second layer embeds reservoirs of depth $H$ between 0.5 and $5 \mathrm{~mm}$ and diameter of $6 \mathrm{~mm}$. The surfactant solution has a concentration of $15 \mathrm{wt} \%$ and, via $\mathrm{D}_{2} \mathrm{O}$ admixture, a negative density difference of $\Delta \rho=-4.35 \%$ is introduced. Thus, droplets float at the ceiling. The droplet diameter is $45 \mu \mathrm{m}$. Top: Schematic illustration of the experimental setup. Bottom: Micrographs of cluster formation after $20 \mathrm{~min}$.

part is a uniform layer of $H_{0}=300 \mu \mathrm{m}$ (see drawing in Fig. 5.43). The droplet swimmers are free to move across the entire area but form clusters only above the wells (lower panel of Fig. 5.43). Here, the cluster spacing and sizes increase with increasing well depth.

5.4.2.2 High-density suspensions in LSM In the previous section, large-scale collective behaviour in unconfined reservoirs was observed. It was shown that a significant buoyancy mismatch is necessary for generating convection rolls and establishing stable clustering. Decreasing the buoyancy mismatch to zero results in independently swimming droplets and suppressed formation of aggregates.

In this respect, light-sheet microscopy is exploited to investigate dense droplet suspensions in three dimensions. Even though no stable aggregates form, the droplets interact, either via direct collision or via autochemotaxis (see Sec. 5.1.4). Although the droplets can move freely on short time scales, caging effects should be visible on long time scales.

These effects are analysed using the MSD and the radii of gyration $R_{g}$. Both parameters are compared for dilute and dense systems. The MSD is determined via eq. (5.11). The radius of gyration is usually used in polymer physics. There, the end-to-end distance of a polymer is compared to its total length to describe the folding capability of the polymer chain. The radius of gyration is defined as [202]

$$
R_{g}^{2}=\frac{1}{N}\left\langle\sum_{k=1}^{N}\left(\mathbf{r}_{k}-\mathbf{r}_{\text {mean }}\right)^{2}\right\rangle
$$

with $N$ as the total number of monomers in the polymer chain, $\mathbf{r}_{k}$ as the location of the $k$ th monomer and $\mathbf{r}_{\text {mean }}$, the centre of mass of the polymer. In other words, it describes the 
averaged squared distance of each monomer to the centre of mass. For a stretched molecule, $R_{g}$ takes large values, while for a strongly folded molecule, $R_{g}$ becomes very small. In the case of droplet trajectories, single droplet positions are used as $\mathbf{r}_{k}$ and the centre of mass of the trajectory as $\mathbf{r}_{\text {mean }}$. The result of $R_{g}$ allows for conclusions on how directed a movement is.

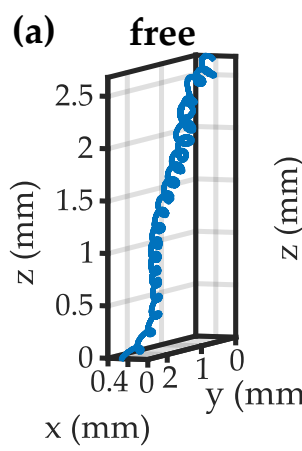

slightly confined

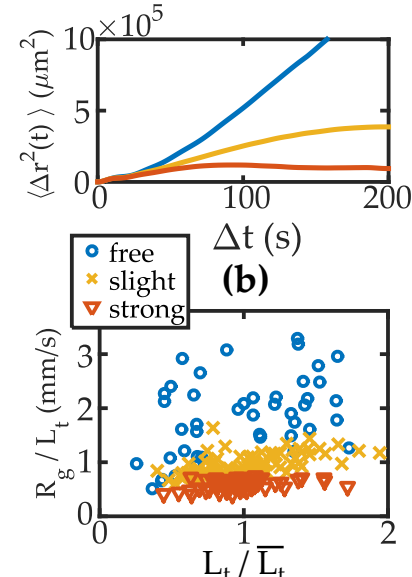

(c)
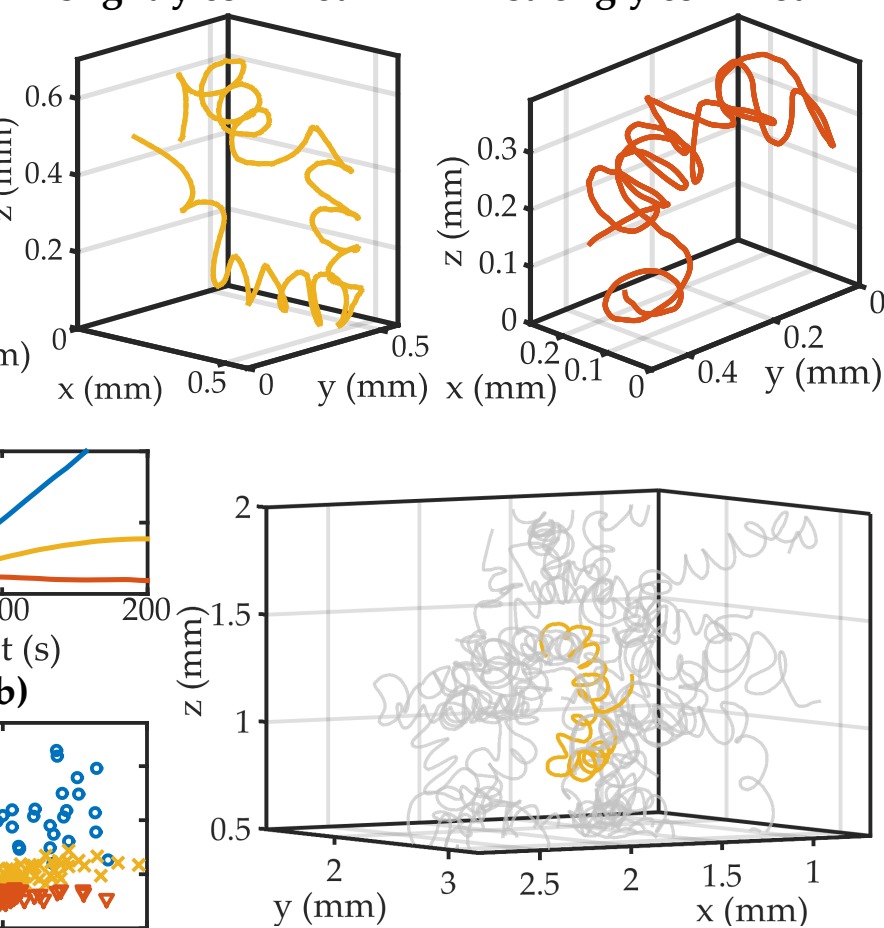

(d)

Fig. 5.44: High density suspensions in LSM. (a) One exemplary trajectory for each case of differing swimmer density. (b) MSDs for different local densities in linear scale. (c) The radius of gyration normalized to the temporal length of the trajectory vs. the normalized track length. The colour code is the same in both graphs. In (b), a stagnation of the MSD is visible for the confined cases, depending on the cage size. The same behaviour is visible in (c). Here, point clouds with differing mean radii of gyration are emerging for the three different cases. (d) In yellow, the caged trajectory is depicted with its nearest neighbours shown in grey.

We traced high-density suspensions with more than 350 droplets using light-sheet microscopy. Fig. 5.44 depicts three single trajectories for qualitatively different cases of local density. The cases are referred to as "free", "slightly confined" and "strongly confined". All trajectories are taken from samples with $c_{s}=7.5 \mathrm{wt} \%$ and have a temporal duration of at least $100 \mathrm{~s}$. The trajectories for the "free" case result from dilute-suspension measurements, while the trajectories in the confined cases result from high-density samples. Figs. $5.44 \mathrm{p}$ and c depict the MSD and the $R_{g}$ for the three different densities. The radius of gyration is normalized to the temporal length $L_{t}$ of the actual trajectory and for the $x$-axis, the temporal length $L_{t}$ is normalized to the mean temporal length $\bar{L}_{t} . \bar{L}_{t}$ includes all trajectories of one case. 
As indicated by the mean squared displacement (s. Fig. 5.44 p), the three different cases show a significantly differing behaviour. The MSD for the free trajectories increases after the characteristic dip due to curling $\left(\Delta t \approx 20 \mathrm{~s}\right.$ ) further with $t^{2}$-dependence. The confined samples show a stagnating MSD for long times. The slight decrease in MSD for the strongly confined case is due to the finite length of the trajectories which bend back on themselves in the confinement.

Regarding the plateau in the MSD as the cross section of a spherical cage, we can obtain estimations for the cage radii and volumes. For the "slightly" confined case, this approximation results in $d_{\text {cage }}=0.62 \mathrm{~mm}$ and $V_{\text {cage }}=0.13 \mathrm{~mm}^{3}$. For the "strongly" confined case, we obtain $d_{\text {cage }}=0.33 \mathrm{~mm}$ and $V_{\text {cage }}=0.02 \mathrm{~mm}^{3}$. Self-evidently, the smaller cage size is found for the stronger confined case. With the retrieved values of the cage volumes a lower bound for the total number $n_{t}$ of droplets in the investigation volume of $3 \times 3 \times 3 \mathrm{~mm}^{3}$ can be calculated. Thus, the total swimmer number in the "slightly" confined case results to $n_{t} \approx 210$ and for the "strongly" confined case $n_{t}$ becomes 450 . Both of these values are far below the maximum total number of droplets traceable in the investigation volume, while already intriguing collective phenomena occur.

The first dip in the MSD at $\Delta t \approx 20 \mathrm{~s}$, however, is unaffected by caging in both confined cases. With resultant radii of $r_{c u r l}=85.3 \mu \mathrm{m}$ and $82 \mu \mathrm{m}$, respectively, reasonable results are found comparable to the trajectories of free swimmers (see Sec. 5.3).

When investigating the radius of gyration with the mentioned normalization for the $x$ - and $y$-axis, point clouds emerge (s. Fig. 5.44 c). Here, the mean values increase with decreasing confinement. $R_{g}$ fluctuates strongly for free swimmers, whereas the data points of the confined cases show only little scatter.

For a better visualization of the caging effects, an example trajectory for the "slightly confined" case is depicted in Fig. 5.44 d. The illustration of the closest neighbours show how the trajectory is purely confined by steric and autochemotactic interactions.

I have demonstrated that a full treatment of dimensionality, geometry and hydrodynamics is necessary to understand the collective dynamics of active swimmers. Inter alia, I have discussed that convective patterns are strongly influenced by the geometry of the system. For active Brownian swimmers [57, 203, 204], clustering should be suppressed by the increasing dimensional degrees of freedom. This stresses the importance of hydrodynamic interactions in our system of more persistent swimmers. Furthermore, the ensuing study of high density suspensions at zero buoyancy in three dimensional light-sheet microscopy shows the suitability of our artificial system for investigating the physics of collective ensembles, e.g. swarms of fish or flocks of birds.

Despite of the complexity of collective features in our experiments, all of these can be well explained by considering and adjusting buoyancy, geometry and hydrodynamics. Here, sys- 
tem-specific parameters, e.g. swimmer morphology or chemistry, do not have to be considered. This is highly relevant for comparison with biological systems. For example, many systems in nature create bioconvective patterns by themselves (e.g. Tetrahymena pyriformis). In other examples, the interaction of the entities with external flows can be found in the species diversity problem as e.g. stated in the Plankton paradox.

I propose this system of active emulsions as a well-suited candidate for comparison with complex biological systems. As the swimmers are perfect spheres and external forces like gravity can be tuned, hydrodynamic modelling is facilitated. The swimmers are highly scalable in numbers and sizes and both, swimmer dynamics and flow fields, can be easily observed and tracked by optical or light-sheet microscopy at all relevant length scales. 



\section{Conclusions}

In the present thesis, manyfold aspects of a specific artificial active droplet system have been outlined and analysed. The basic ingredients are oil droplets of the nematic liquid crystal $5 \mathrm{CB}$ immersed in an ionic surfactant solution of TTAB. In a spontaneous process, the droplets undergo micellar solubilization and at surfactant concentrations well above the CMC, the droplet starts to self-propel. The necessary symmetry breaking results from inhomogeneities of surfactant coverage on the droplet's interface and self-sustained Marangoni flows arise.

In today's scientific community, it is of high interest to reconstruct biological phenomena especially the mobility and the collective behaviour of living matter. In this respect, the system of active emulsions is well suited to mimic biological systems and to tackle so far unanswered questions. Furthermore, the system provides the versatility to serve as an experimental benchmark for numerical simulations and offers the ability to explore active systems at high complexity.

Under that aspect, the presented system is not just characterized in terms of its fundamental propulsion mechanism but also the controllability of various system parameters and thus, inherent characteristic properties of the system are shown. Varying parameters like the liquid crystalline phase, the number density, dimensionality, driving force or the buoyancy result in the control of properties like the persistence of motion, (auto)chemotactic behaviour, characteristic propulsion patterns and collective dynamics, e.g. clustering and caging. Thus, a large extent of the parameter space of interest was covered and various important properties reminiscent of biological systems were shown in the present thesis.

First, experiments were conducted as a characterization of the underlying propulsion mechanism. The importance of the solubilization mechanism and the resulting Marangoni flows have been described in detail in the theoretical section of this thesis. Even though it was not possible to prove these assumptions on the molecular level, I executed individual, inde-

pendent measurements to strengthen the beforehand made assumptions of the fundamental mechanism.

In solubilization kinetics, two different pathways are described, the molecular and the micellar pathway. The first assumption made is that only the molecular pathway results in depletion patterns of surfactants, giving rise to self-sustained motion. Thus, via investigating the solubilization rates, a deeper understanding of the kinetics is gained. For this, we changed either the viscosity or the ion amount of the surfactant solution. In the case of $5 \mathrm{CB} / \mathrm{TTAB}$, increasing the viscosity resulted in decreased solubilization rates, while the increased ion amount resulted in increasing solubility rates. Both aspects strongly suggest that the molecular pathway contributes significantly to the solubilization kinetics. Furthermore, from considering the solubilization rate and applying the theoretical scheme of the Todorov 
model, we deduced the thickness of the reactive layer in which the 5CB molecules are incorporated into the micelles. Thus, we verified the layer thicknesses to be much smaller than the droplet radius, as expected for molecular solubility.

In a basic experiment, the one-dimensional velocity of the droplets has been extracted from measurements in square capillaries. The course of the velocity depending on the surfactant concentration explicitly follows the theoretical assumption based on the driving parameter $\beta$, with values between 5 and $25 \mu \mathrm{m} / \mathrm{s}$ for droplets of $50 \mu \mathrm{m}$ diameter. Furthermore, the influence of temperature and of the liquid crystal phase transition on the propulsion has been investigated. It was shown that an increasing temperature results in increasing droplet velocities, while the transition to the isotropic phase does not inhibit the movement.

When introducing the Marangoni-flow powered model system, the assumption has been made that even minor inhomogeneities in interfacial tension $\Delta \gamma$ along the droplet surface are sufficient to generate self-propulsion on the order of micrometres per second. Considering the viscous forces and the energy dissipation along the droplet interface results in a relation for the gradient in surface tension. Taking the measured velocities and the physical properties of the system into account, $\nabla \gamma$ is calculated and values in the range of 0.3 to $2.5 \mathrm{mN} / \mathrm{m}^{2}$ are deduced. The gradient along one droplets hemisphere relates to the difference in interfacial tension between leading and rear end. The deduced values are compared with the equilibrium surfactant coverage, extracted by pendant drop measurements. Concluding, relative surfactant inhomogeneities on the order of $10^{-4}$ are sufficient for droplet propulsion in the micron per second range. This is in perfect accordance with the assumptions for artificial swimmers powered by Marangoni-flow.

In the theoretical introduction, chemotaxis has been outlined as an important mechanism of biological phenomena. The propulsion mechanism of the droplet system itself implies a directed motion due to concentration gradients. Experiments proved a chemotactic behaviour of the LC droplets that even leads to maze-solving schemes. These experiments thus pave the way towards future applications in microfluidic devices, e.g. directed sorting and drug delivery.

As a second important aspect that is closely related to biological systems, we found our droplets to be autochemotactic. This means that the droplet senses trails generated by itself or other droplets and avoids these on time scales of a few tens of seconds. So far, this behaviour has not been reported on in artificial systems.

In artificial swimmer systems, it is important to determine the squirmer type. Based on nature's equivalents, it is distinguished between neutral, pusher or puller squirmers. Via particle shadow velocimetry, it is possible to investigate the flow profile for the $5 \mathrm{CB}$ droplets. Thus, we clarified that the artificial droplets belong to the neutral squirmer class as was expected for spherically symmetric droplets. The implications of these findings are particularly discussed 
in the studies on interactions of the droplets with walls and with other droplet entities.

In two dimensions, the velocity dependence on the surfactant concentration is determined, similar to the one-dimensional measurements. Furthermore, we investigated the influence of the droplet diameter on the propulsion speed. A theoretical scheme, taking the solubilization rate, the micelle size, the droplet radius and the viscosity ratios into account, is applied for comparison with the experimental results. A linear increase of velocity with the diameter is predicted, with the slope depending on the solubilization rate and micelle size that is determined by the surfactant concentration. Our experimentally obtained values are in good agreement with this prediction up to droplet diameters of $60 \mu \mathrm{m}$. For larger droplet diameters, the velocity converges to a mean velocity of $v=37 \mu \mathrm{m} / \mathrm{s}$.

The implication of diameter-dependent propulsion on the movement threshold i tested to determine the critical surfactant concentration $c_{c r i t}$ for propulsion. In these experiments, a lower bound for the critical surfactant concentration has been determined to $c_{\text {crit }}=3 \mathrm{wt} \%$, resulting in a critical driving parameter of $\beta_{c}=0.35 \mu \mathrm{m}^{-1}$.

The characterization of the active droplets confirmed the theoretical assumptions based on molecular solubility, surfactant depletion and the creation of Marangoni flows. Furthermore, the course of the droplet velocities depending on surfactant concentration follows the predicted behaviour of the driving parameter $\beta$, while the diameter dependence is well described by the theoretical framework outlined above. As a next step, individual system parameters like dimensionality, number density and buoyancy are varied. The controllability of relevant system properties like motility and collective behaviour shows the usability of this active squirmer approach for mimicking biological systems and reproducing their dynamics.

Nature exploits various kinds of attractive interactions with boundaries, e.g. for biofilm formation. The gained information about the hydrodynamic flow field suggest strong attractive interactions of the swimmers with obstacles. And indeed, distinct hydrodynamic attraction to walls was found, leading to rectification dynamics and an extended detention time on walls. When confining a droplet in microchannels with different widths, the compression and the back action of the flow profile onto the droplet leads to an oscillatory switching between the walls for a critical droplet diameter to width ratio.

An extensive investigation in Hele-Shaw kind geometries was performed to unravel the reasons for a prominent curling motion exhibited by the nematic liquid crystal droplets. At first glance, this behaviour is reminiscent of the swimming of flagellates close to boundaries, where a coupling of hydrodynamic flow to the interface results in a torque onto the cell body. In the artificial system, the torque is generated by the interplay of the nematic matrix and the internal convection. Here, the topological defect is deflected away from the droplet apex at a finite angle $\Theta_{s}$ towards the droplet equator. We found that the magnitude of $\Theta_{s}$ depends on 
the swimmer's velocity by using polarized microscopy. Moreover, we could show that the distortion of the nematic field creates the curling motion of the droplets, resulting from an unbalanced velocity profile on the two droplet's hemispheres. Via particle shadow velocimetry, we could resolve this left-right asymmetry in the flow profile. When tuning the temperature and thus, switching the droplet from the nematic to the isotropic phase, the curling motion ceases immediately. This transition gives rise to a highly persistent ballistic motion and offers the possibility to switch the movement behaviour of the droplets externally.

The curling motion at low surfactant concentrations has been furthermore compared with meandering motion at increased surfactant concentrations. Even though the time scales for both phenomena are comparable, the magnitude of autochemotactic signalling in the meandering case is substantially higher. The resultant inhibition of self-crossing poses the characteristic motion to change from the curling to the meandering mode.

To show that the curling motion is independent of the confinement, the droplets were also traced in three dimensions by light-sheet microscopy. We recorded helical trajectories, caused by an interplay of torque and autochemotactic effects. Meandering motion was not observed in three dimensions, thus emphasizing the unfavourable displacement of the defect necessary for this propulsion mode. An extended study on three dimensional propelling presented changes in pitch, period and helical radius in dependence of the surfactant concentration up to $c_{s}=15 \mathrm{wt} \%$. For higher surfactant concentrations, the helical motion is suppressed and gives way for erratic propulsion characteristic, resembling active Brownian motion. The results confirm the findings that have been made in two dimensions.

These experiments emphasize the influence of temperature, surfactant concentration and autochemotaxis on the characteristic behaviour of the swimmers. Adjusting these parameters accordingly allows to choose and define the behaviour of the droplet swimmers.

In the last part of the thesis, collective interactions are addressed to emphasize the capability of this active emulsion system to investigate large scale phenomena occurring in nature, e.g. schools of fish or the Plankton paradox. In a first setting, suspensions at high densities are investigated in cylindrical, unconfined geometries. Over time, these suspension demix into convection-roll stabilized clusters and a dilute gas-like phase. The clusters develop by collisions between particles which get arrested and organize in a hexagonal layer. The convection roll is then created by a superposition of the single swimmers' flows. The sedimenting swimmers in the cluster centre point upwards, while swimmers on the boundaries compress the cluster with a slightly inwards tilted orientation. We verified this arrangement by using polarized microscopy and particle tracking velocimetry. An estimate of the flow velocity of the convection-roll and the boundary swimmers strengthens the compression argument. Different scaling laws of the cluster size and the cluster spacing are evaluated and show a good agreement with the systematic assumptions for the influence of the convection roll on the clustering behaviour. 
Two observations confirm the proposed mechanism: First, confinement suppresses the convection roll and thus, inhibits clustering. Second, a certain minimum buoyancy has to act on the droplets to establish the equilibrium between the sedimentation and the lifting force due to droplet propulsion.

The latter assumption is tested by admixture of heavy water $\mathrm{D}_{2} \mathrm{O}$ to the surfactant solution, which allows for a continuous changing of the buoyancy settings. These measurements revealed that a critical density mismatch between the droplet swimmers and the surfactant solution has to exist to prevent the droplets from escaping regions of cluster formation. Furthermore, a critical ratio of the swimming speed of the droplets and the sedimentation velocities can be obtained from the data at hand. Thus, it is derived that clustering is inhibited if the droplet speed exceeds the sedimentation speed by approximately a factor of two. Since this value is higher than unity the influence of hydrodynamic interactions of the entities is emphasized once more.

The implication of buoyancy adjustments on the collective behaviour is further investigated in light-sheet microscopy. The density mismatch is tuned to zero and free-floating droplet suspensions at high densities are observed in three dimensions. Even though cluster formation is inhibited, droplets still interact with each other by hard-core repulsion or via negative autochemotaxis, resulting in caging dynamics. These caging effects were analysed by statistics of the mean squared displacement and the radius of gyration.

Via extensive studies, our artificial swimmer system has been characterized in detail, particularly with regard to the propulsion mechanism. Many system specific aspects, e.g. autochemotaxis, wall interactions, curling motion, large-scale collective behaviour and the helical three-dimensional movement were investigated and can be controlled on purpose. Moreover, many of these characteristics can be adapted to systems of biological swimmers. Furthermore, the long active period of the active emulsion system at hand enables us to mimic various biological phenomena, also on long time scales. 



\section{Ongoing research}

Various aspects have been touched along the way of experimental investigations but open questions still prevail. For instance, a quantitative determination of the surfactant micelles/LC molecule concentration in the vicinity of droplets could not be performed. This lack prevents a direct measure of the autochemotactic signalling. Techniques like Schlieren microscopy or spectroscopy might give further insights into the influences of these quantities, e.g. micellar diffusion and/or autochemotactic behaviour.

Furthermore, optical traps can be exploited to fix a droplet at a specified position for measurements. Further applications for an optical trap, preferably with active positioning of two foci, are given by the investigation of droplet-droplet interactions or local creation of director distortions. The changes in the propulsion pattern, which are caused by the interplay of the nematic field with internal currents are of particular interest.

The impact of the intrinsic liquid crystal properties on the propulsion was discussed in detail. Thus, the interaction of the internal flow field with the nematic matrix resulted in curling trajectories in 2D and in helical trajectories in 3D. However, besides switching the droplets into the isotropic phase, also admixture of a chiral dopant to the nematic droplets is possible. In the chiral nematic phase, the director field forms a helical superstructure. This specific droplet kind has been so far neglected in the investigations. In preliminary measurements of two dimensional propulsion at dilute swimmer densities, $50 \mu \mathrm{m}$ sized chiral droplets showed erratic motion without stable curling modes. Yet, we observed that these droplets do not only translate but also rotate while moving. Decreasing the droplet diameter to a few times the pitch $(d \approx 20 \mu \mathrm{m})$ resulted in curling trajectories, similar to pure nematic droplets but with lower stability in curl radii.

Introducing a large number of these chiral droplets into unconfined reservoirs, preliminary measurements showed an interesting feature in the clustering dynamics. We found that clusters formed by chiral nematic droplets show a rotation of the whole cluster due to the rotation of the single droplets. This adds a tangential velocity to the cluster, with its orientation depending on the handedness of the enantiomer. This behaviour is similar to observations of aggregates of the bacterium Thiovulum majus [205].

External fields, electric or magnetic, are often applied in LC experiments. For the droplet propulsion, the basic idea lies in altering and controlling the nematic director orientation across the droplet by external fields. Subsequently, the influence of this intrusion on the propulsion behaviour can be observed. The ionic character of the surfactant solution has so far prevented the generation of sufficiently high electric field strengths in PDMS devices. In particular, we have observed arcing across the insulating polymer. This problem might be solved in the near future with more sophisticated channel designs and materials. 
In the case of magnetic fields, first results in a simple experimental geometry have been obtained. Interestingly, a ceasing of curling or helical motion perpendicular to the field orientation is observed at a field strength of roughly $100 \mathrm{mT}$. This offers another possibility when a local influence of the propulsion and diffusion characteristics is demanded. Moreover, this modification paves the way towards additional insights into the elastic structure of the nematic droplet material.

Hitherto, we have been able to explore a plethora of phenomena using basic experimental geometries. However, the versatility of PDMS and photolithography is by far not fully exploited yet. In literature, rectification schemes or diffusion enhancing layouts are often mentioned, mostly investigated in simulations [179, 206, 207]. The LC system that provides long active periods and an easy manipulation of the diffusivity and droplet dimensions is a perfect candidate for comparative experimental studies.

High density systems are difficult to investigate in two dimensions due to fast depletion of empty micelles. A promising application bases on double layered microfluidic structures, permitting a constant replenishment of surfactant micelles by a constant fluid flow below the observation chamber, separated by a permeable membrane. A few studies on bacterial films have been mentioned in literature [208].

The defect topology inside a nematic droplet can be varied by changing the anchoring conditions (cf. Fig. 2.9). Based on the results presented in Sec. 5.2.5, this implication could lead to further interesting swimming modes.

Double emulsions provide even more possibilities [74, 209]. In this case, the differing anchoring conditions on the two interfaces and the interaction with the fluid flows can result in intriguing phenomena. Up to now, only resting droplets and the different emerging director and defect patterns have been studied on. The effect of propulsion or flow in general is not investigated extensively in literature yet [123].

Even though many schemes for analysing the recorded data have already been employed, further recipes taken from literature might be adapted. For interacting droplets, the concepts of transfer entropy and a scheme to deduce the interaction potential are very promising. Both approaches use the experimental trajectories of droplets already at hand.

The transfer entropy is a tool to obtain the mutual information exchange between different droplets [210]. For an interacting pair, this might result in information about which of the droplets defines the following path taken by the pair. This resembles the movement of flocks, where usually an individual or a small group of entities determines the path of the whole flock. Testing this procedure on single pairs and evolving a collective analysis might give interesting insights into the collective behaviour. 
The second idea was proposed by Jenkins et al. [211]. Here, the extraction of a pairwise interaction potential from multi-particle trajectory data sets was derived. This procedure was tested on simulated data sets that even contained noise for an optimal reconstruction of experimental conditions.

Most of the proposed ideas relate to two-dimensional settings. Especially three-dimensional investigations in the light-sheet microscope that have recently evolved pose many interesting questions. In suspensions of Tetrahymena pyriformis, an upwards swimming organism that is denser than the surrounding liquid, large-scale three-dimensional convective structures are formed [50]. The adjustment of the surrounding liquid density in our system allows us to change the buoyancy settings at will. Moreover, the autochemotactic signalling of the droplets provides another option to generate collective fluxes in dense suspensions of swimmers.

First experiments on wall interactions in three dimensions suggest that hydrodynamic forces are increasing with increasing confinement. Well controlled three dimensional obstacles facilitate more distinct studies on these interactions. Furthermore, particle tracking velocimetry could provide full three-dimensional flow profiles.

For mimicking nature-like scenarios, a refractive index matching material in the form of colloids shows promising features [212]. Most organisms in nature live in complex geometries and with the proposed material at hand, the production of artificial soil is feasible. With lightsheet microscopy not influenced by strong scattering of light, tracking the artificial swimmers as a benchmark system is possible.

A general perspective is the adjustment of the ingredients of the system to ensure longer acquisition times. So far, all proposed mechanisms in active emulsions run, sooner or later, out of "fuel". The major aim is to set up a system with an enabled resupplying of fuel or recovery of reactive molecules during the active period.

One idea for longer measurement periods bases on switchable molecules, e.g. an azo surfactant that can be switched between a cis and a trans conformation. A feasible scheme for such a molecule is the incorporation of a catalysing species inside the droplet. Here, the azo surfactant is switched into a different state when the two species get into contact at the droplet interface. If the surfactant is switched to a less surface-affine state, an increase in interfacial tension results and Marangoni flows arise. If this surfactant equilibrates on small time scales back into the initial state, an infinite supply of fuel for the propulsion is provided. With this method, active emulsions can mimic metabolic organisms and hence, would literally be micro-swimmers. 



\section{References}

[1] E. Lauga and T. R. Powers, Reports Prog. Phys. 72, 096601 (2009).

[2] H. C. Berg and D. A. Brown, Nature 239, 500 (1972).

[3] K. Yoshimura and R. Kamiya, Plant Cell Physiol. 42, 665 (2001).

[4] S. Herminghaus, C. C. Maass, C. Krüger, S. Thutupalli, L. Goehring, and C. Bahr, Soft Matter 10, 7008 (2014)

[5] J. Elgeti, R. G. Winkler, and G. Gompper, Rep. Prog. Phys. 78, 056601 (2015).

[6] S. Thutupalli, Towards Autonomous Soft Matter Systems, Ph.D. thesis, University of Göttingen (2014).

[7] "Ant mill," http://studytub.com/wp-content/uploads/2016/01/Army-Ants-deathcircle.jpg, date accessed 2016-08-15.

[8] G. A. Voth, B. Bigger, M. R. Buckley, W. Losert, M. P. Brenner, H. A. Stone, and J. P. Gollub, Phys. Rev. Lett. 88, 234301 (2002).

[9] D. Yamada, T. Hondou, and M. Sano, Phys. Rev. E. Stat. Nonlin. Soft Matter Phys. 67, 040301 (2003).

[10] R. Dreyfus, J. Baudry, M. L. Roper, M. Fermigier, H. A. Stone, and J. Bibette, Nature 437, 862 (2005).

[11] A. Ghosh and P. Fischer, Nano Lett. 9, 2243 (2009).

[12] T. Qiu, T.-C. Lee, A. G. Mark, K. I. Morozov, R. Münster, O. Mierka, S. Turek, A. M. Leshansky, and P. Fischer, Nat. Commun. 5, 5119 (2014).

[13] J. Anderson, Annu. Rev. Fluid Mech. 21, 61 (1989).

[14] H. R. Jiang, N. Yoshinaga, and M. Sano, Phys. Rev. Lett. 105, 1 (2010).

[15] G. Volpe, I. Buttinoni, D. Vogt, H.-J. Kümmerer, and C. Bechinger, Soft Matter 7, 4 (2011).

[16] I. Buttinoni, G. Volpe, F. Kümmel, G. Volpe, and C. Bechinger, J. Phys. Condens. Matter 24, 284129 (2012).

[17] S. Thutupalli, R. Seemann, and S. Herminghaus, New J. Phys. 13, 073021 (2011).

[18] Z. Izri, M. N. van der Linden, and O. Dauchot, Phys. Rev. Lett. 1, 4 (2014).

[19] C. C. Maass, C. Krüger, S. Herminghaus, and C. Bahr, Annu. Rev. Condens. Matter Phys. 7, 171 (2016). 
[20] E. Lauga and A. M. J. Davis, J. Fluid Mech. 705, 120 (2012).

[21] E. M. Purcell, Am. J. Phys. 45, 3 (1977).

[22] E. Guyon, J.-P. Hulin, L. Petit, and C. D. Mitescu, Physical Hydrodynamics, 2nd ed. (Oxford University Press, 2015).

[23] L. Xie, T. Altindal, S. Chattopadhyay, and X.-L. Wu, Proc. Natl. Acad. Sci. U. S. A. 108, 2246 (2010).

[24] E. Lauga, W. R. DiLuzio, G. M. Whitesides, and H. A. Stone, Biophys J 90, 400 (2006).

[25] J. Gray, J. Exp. Biol. 32, 775 (1955).

[26] H. C. Berg and L. Turner, Biophys. J. 58, 919 (1990).

[27] D. M. Woolley, Reproduction 126, 259 (2003).

[28] W. R. DiLuzio, L. Turner, M. Mayer, P. Garstecki, D. B. Weibel, H. C. Berg, and G. M. Whitesides, Nature 435, 1271 (2005).

[29] S. Kudo, N. Imai, M. Nishitoba, S. Sugiyama, and Y. Magariyama, FEMS Microbiol. Lett. 242, 221 (2005),

[30] V. B. Shenoy, D. T. Tambe, A. Prasad, and J. A. Theriot, Proc. Natl. Acad. Sci. U. S. A. 104, 8229 (2007).

[31] J. P. Celli, B. S. Turner, N. H. Afdhal, S. Keates, I. Ghiran, C. P. Kelly, R. H. Ewoldt, G. H. McKinley, P. So, S. Erramilli, and R. Bansil, Proc. Natl. Acad. Sci. U. S. A. 106, 14321 (2009).

[32] M. Molaei, M. Barry, R. Stocker, and J. Sheng, Phys. Rev. Lett. 113, 1 (2014).

[33] H. Jennings, Am. Nat. 35, 369 (1901).

[34] K. W. Foster and R. D. Smyth, Microbiol. Rev. 44, 572 (1980).

[35] R. N. Bearon, J. Math. Biol. 66, 1341 (2013).

[36] H. C. Crenshaw, Helical Orientation - A novel mechanism for the orientation of microorganisms (Springer-Verlag Berlin Heidelberg, 1990) pp. 361-386.

[37] H. C. Crenshaw, Bull. Math. Biol. 55, 197 (1993).

[38] J. Hu, A. Wysocki, R. G. Winkler, and G. Gompper, Sci. Rep. 5, 9586 (2015).

[39] S. van Teeffelen and H. Löwen, Phys. Rev. E - Stat. Nonlinear, Soft Matter Phys. 78, 2 (2008).

[40] S. van Teeffelen, U. Zimmermann, and H. Löwen, Soft Matter 5, 4510 (2009). 
[41] B. M. Friedrich and F. Jülicher, Phys. Rev. Lett. 103, 1 (2009).

[42] R. Wittkowski and H. Löwen, Phys. Rev. E - Stat. Nonlinear, Soft Matter Phys. 85, 1 (2012)

[43] J. Dunkel, S. Heidenreich, K. Drescher, H. H. Wensink, M. Bär, and R. E. Goldstein, Phys. Rev. Lett. 110, 1 (2013)

[44] H. Wioland, F. G. Woodhouse, J. Dunkel, J. O. Kessler, and R. E. Goldstein, Phys. Rev. Lett. 110, 1 (2013).

[45] E. Lushi, H. Wioland, and R. E. Goldstein, Proc. Natl. Acad. Sci. U. S. A. 111, 9733 (2014).

[46] W. M. Durham, E. Climent, M. Barry, F. De Lillo, G. Boffetta, M. Cencini, and R. Stocker, Nat. Commun. 4, 2148 (2013).

[47] A. Sokolov and I. S. Aranson, Phys. Rev. Lett. 103, 2 (2009)

[48] S. Rafaï, L. Jibuti, and P. Peyla, Phys. Rev. Lett. 104, 1 (2010).

[49] J. Platt, Science 133, 1766 (1961).

[50] T. J. Pedley and J. O. Kessler, Annu. Rev. Fluid Mech. 24, 313 (1992).

[51] N. A. Hill and T. J. Pedley, Fluid Dyn. Res. 37, 1 (2005)

[52] G. Hutchinson, Am. Nat. 95, 137 (1961).

[53] M. Scheffer, S. Rinaldi, J. Huisman, and F. J. Weissing, Hydrobiologia 491, 9 (2003).

[54] F. D'Ovidio, S. De Monte, S. Alvain, Y. Dandonneau, and M. Lévy, Proc. Natl. Acad. Sci. U. S. A. 107, 18366 (2010).

[55] J. R. Howse, R. A. L. Jones, A. J. Ryan, T. Gough, R. Vafabakhsh, and R. Golestanian, Phys. Rev. Lett. 99, 8 (2007).

[56] S. Duhr and D. Braun, Proc. Natl. Acad. Sci. U. S. A. 103, 19678 (2006).

[57] I. Buttinoni, J. Bialké, F. Kümmel, H. Löwen, C. Bechinger, and T. Speck, Phys. Rev. Lett. 110, 1 (2013).

[58] F. Kümmel, B. Ten Hagen, R. Wittkowski, I. Buttinoni, R. Eichhorn, G. Volpe, H. Löwen, and C. Bechinger, Phys. Rev. Lett. 110, 1 (2013).

[59] B. ten Hagen, F. Kümmel, R. Wittkowski, D. Takagi, H. Löwen, and C. Bechinger, Nat. Commun. 5, 4829 (2014)

[60] T. Toyota, H. Tsuha, K. Yamada, K. Takakura, T. Ikegami, and T. Sugawara, Chem. Lett. 35, 708 (2006). 
[61] M. M. Hanczyc, T. Toyota, T. Ikegami, and N. Packard, J. Am. Chem. Soc. 129, 9386 (2007).

[62] T. Toyota, N. Maru, M. M. Hanczyc, T. Ikegami, and T. Sugawara, J. Am. Chem. Soc. 131, 5012 (2009).

[63] T. Banno, R. Kuroha, and T. Toyota, Langmuir 28, 1190 (2012)

[64] T. Ban, T. Yamagami, H. Nakata, and Y. Okano, Langmuir 29, 2554 (2013).

[65] K. Peddireddy, P. Kumar, S. Thutupalli, S. Herminghaus, and C. Bahr, Langmuir 28, $12426(2012)$

[66] A. Sengupta, Nematic Liquid Crystals and Nematic Colloids in Microfluidic Environment, Ph.D. thesis, University of Göttingen (2011).

[67] F. Reinitzer, Monatshefte für Chemie 9, 421 (1888).

[68] O. Lehmann, Zeitschrift für Phys. Chemie 4, 462 (1889).

[69] S. Chandrasekhar, Liquid Crystals (Cambridge University Press, 1994).

[70] S.-T. Wu, Appl. Opt. 26, 3434 (1987).

[71] P. Oswald and P. Pieranski, Nematic and Cholesteric Liquid Crystals (Taylor \& Francis, 2005).

[72] M. Miesowicz, Nature 158, 27 (1946).

[73] C. Bahr, Phys. Rev. E - Stat. Nonlinear, Soft Matter Phys. 73, 1 (2006).

[74] T. Lopez-Leon and A. Fernandez-Nieves, Colloid Polym. Sci. 289, 345 (2011).

[75] M. J. Rosen and J. Kunjappu, Surfactants and Interfacial Phenomena, 3rd Edition. (WileyInterscience, 2012) p. 464.

[76] A. A. Peña and C. A. Miller, Adv. Colloid Interface Sci. 123-126, 241 (2006).

[77] S. Michelin, E. Lauga, and D. Bartolo, Phys. Fluids 25, 1 (2013).

[78] J. M. Yeomans, D. O. Pushkin, and H. Shum, Eur. Phys. J. Spec. Top. 223, 1771 (2014).

[79] J. R. Blake, J. Fluid Mech. 46, 199 (1971).

[80] J. J. Molina, Y. Nakayama, and R. Yamamoto, Soft Matter 9, 4923 (2013).

[81] T. Ishikawa and T. J. Pedley, Phys. Rev. Lett. 100, 1 (2008).

[82] M. T. Downton and H. Stark, J. Phys. Condens. Matter 21, 204101 (2009).

[83] R. Brown, Philos. Mag. 4, 161 (1828). 
[84] A. Einstein, Ann. Phys. 17, 549 (1905).

[85] A. Einstein, Ann. Phys. 19, 371 (1906).

[86] M. von Smoluchowski, Ann. Phys. 326, 756 (1906)

[87] F. Schweitzer, Browning Agents and Active Particles (Springer Complexity, 2003).

[88] R. Mazo, Brownian Motion: Fluctuations, Dynamics and Applications, Vol. 6 (Oxford Science Publications, 2008).

[89] T. Vicsek and A. Zafeiris, Phys. Reports; Collect. motion 517, 71 (2012).

[90] A. G. Chmielewski, Mol. Cryst. Liq. Cryst. 132, 339 (1986).

[91] Springer Materials, "Landolt-Börnstein Database," date accessed 2016-05-09.

[92] R. Mattern, “5CB - Molecular structure," date accessed 2016-05-11.

[93] J. Yoshioka, F. Ito, and Y. Tabe, Soft Matter 12, 2400 (2016).

[94] Sigma Aldrich, "Myristyltrimethylammonium bromide," (), date accessed 2016-05-11.

[95] Sigma Aldrich, "Hexadecyltrimethylammonium bromide," (), date accessed 2016-0511.

[96] Sigma Aldrich, "Sodium dodecyl sulfate," (), date accessed 2016-05-11.

[97] C. R. Haramagatti, Structure, Dynamics and Phase Behaviors of Cationic Micellar Solutions, Ph.D. thesis, University of Göttingen (2006).

[98] K. Peddireddy, Liquid Crystals in Aqueous Ionic Surfactant Solutions: Interfacial Instabilities $\mathcal{E}$ Optical Applications, Ph.D. thesis, University of Göttingen (2014).

[99] R. Seemann, M. Brinkmann, T. Pfohl, and S. Herminghaus, Rep. Prog. Phys. 75, 16601 (2012)

[100] J. C. McDonald and G. M. Whitesides, Acc. Chem. Res. 35, 491 (2002)

[101] I. Wong and C. M. Ho, Microfluid. Nanofluidics 7, 291 (2009).

[102] G. Muschiolik, Curr. Opin. Colloid Interface Sci. 12, 213 (2007)

[103] T. G. Mason and J. Bibette, Phys. Rev. Lett. 77, 3481 (1996).

[104] I. Kobayashi, S. Mukataka, and M. Nakajima, Langmuir 21, 7629 (2005).

[105] P. B. Umbanhowar, V. Prasad, and D. A. Weitz, Langmuir 16, 347 (2000).

[106] T. Thorsen, R. W. Roberts, F. H. Arnold, and S. R. Quake, Phys. Rev. Lett. 86, 4163 (2001)

[107] S. L. Anna, N. Bontoux, and H. A. Stone, Appl. Phys. Lett. 82, 364 (2003) 
[108] G. F. Christopher and S. L. Anna, J. Phys. D. Appl. Phys. 40, R319 (2007).

[109] B. D. Hamlington, B. Steinhaus, J. J. Feng, D. Link, M. J. Shelley, and A. Q. Shen, Liq. Cryst. 34, 861 (2007).

[110] M. Belloul, J. F. Bartolo, B. Ziraoui, F. Coldren, V. Taly, and A. I. El Abed, Appl. Phys. Lett. 103, 1 (2013).

[111] Z. Nie, M. Seo, S. Xu, P. C. Lewis, M. Mok, E. Kumacheva, G. M. Whitesides, P. Garstecki, and H. A. Stone, Microfluid. Nanofluidics 5, 585 (2008).

[112] W. Lee, L. M. Walker, and S. L. Anna, Phys. Fluids 21 (2009).

[113] T. Young, Philos. Trans. R. Soc. London 95, 65 (1805).

[114] T. Fu, Y. Wu, Y. Ma, and H. Z. Li, Chem. Eng. Sci. 84, 207 (2012).

[115] C. Zhou, P. Yue, and J. J. Feng, Phys. Fluids 18 (2006).

[116] A. S. Utada, A. Fernandez-Nieves, H. A. Stone, and D. A. Weitz, Phys. Rev. Lett. 99, 1 (2007).

[117] T. Cubaud and T. G. Mason, Phys. Fluids 20, 1 (2008).

[118] T. Ward, M. Faivre, M. Abkarian, and H. A. Stone, Electrophoresis 26, 3716 (2005).

[119] T. Cubaud, M. Tatineni, X. Zhong, and C. M. Ho, Phys. Rev. E - Stat. Nonlinear, Soft Matter Phys. 72, 28 (2005).

[120] J. Lee, C. Park, and G. M. Whitesides, Anal. Chem. 75, 6544 (2003).

[121] I. Dierking, Textures of Liquid Crystals (Wiley-VCH, 2003).

[122] MicroscopyU, "Introduction to Polarized Light Microscopy," date accessed 2016-06-01.

[123] A. Fernández-Nieves, D. R. Link, M. Márquez, and D. A. Weitz, Phys. Rev. Lett. 98, 1 (2007).

[124] J. Huisken and D. Y. R. Stainier, Development 136, 1963 (2009).

[125] A. Voie, D. Burns, and F. Spelman, J. Microsc. 170, 229 (1993).

[126] J. Huisken, J. Swoger, F. Del Bene, J. Wittbrodt, and E. H. K. Stelzer, Science 305, 1007 (2004).

[127] K. Greger, J. Swoger, and E. H. K. Stelzer, Rev. Sci. Instrum. 78, 1 (2007)

[128] M. Abramowitz, K. R. Spring, H. E. Keller, and M. W. Davidson, Biotechniques 33, 772 (2002). 
[129] C. D. Meinhart, S. T. Wereley, and M. H. B. Gray, Meas. Sci. Technol. 11, 809 (2000).

[130] K. R. Spring and M. W. Davidson, "Properties of Microscope Objectives," date accessed 2016-05-03.

[131] P. A. Santi, J. Histochem. Cytochem. 59, 129 (2011).

[132] "Schematic of Gaussian Beam, WikiMedia," date accessed 2016-05-03.

[133] E. Hecht, Optics (Addison Wesley, 2001).

[134] M. Humar and I. Musevic, Opt. Express 18, 26995 (2010).

[135] K. Peddireddy, V. S. R. Jampani, S. Thutupalli, S. Herminghaus, C. Bahr, and I. Musevic, Opt. Express 21, 30233 (2013).

[136] Sigma Aldrich, “NileRed," (), date accessed 2016-05-03.

[137] Thermo Fisher Scientific, "Nile Red - Fluorescence Spectrum," date accessed 2016-05-03.

[138] AHF, “605 LP ET Longpass Filter," date accessed 2016-05-03.

[139] G. Klös, “3D tracking of active micro-swimmers,” (2016), Master Thesis, University of Göttingen.

[140] P. B. Chapple, Opt. Eng. 33, 2461 (1994).

[141] H. van de Hulst, Light scattering by small Particles (John Wiley \& Sons, 1957).

[142] S. Suzuki and K. Abe, Comput. Vision, Graph. Image Process. 46, 32 (1985).

[143] J. Crocker and D. Grier, J. Colloid Interface Sci. 179, 298 (1996).

[144] Y. Cheng, IEEE Trans. Pattern Anal. Mach. Intell. 17, 790 (1995).

[145] R. J. Adrian, Annu. Rev. Fluid Mech. 23, 261 (1991).

[146] J. G. Santiago, S. T. Wereley, C. D. Meinhart, D. J. Beebe, and R. J. Adrian, Exp. Fluids 25, $316(1998)$

[147] A. Adamczyk and L.Rimai, Exp. Fluids 6, 373 (1988).

[148] A. Prasad, Curr. Sci. 79, 51 (2000).

[149] J. Estevadeordal and L. Goss, 43rd AIAA Aerosp. Sci. Meet. Exhib. ill, 1 (2005).

[150] W. Thielicke and E. J. Stamhuis, J. Open Res. Softw. 2, e30 (2014)

[151] E. J. Thielicke, W., Stamhuis, “PIVlab - Time-Resolved Digital Particle Image Velocimetry Tool for MATLAB (version: 1.4)," (2015). 
[152] P. D. Todorov, P. A. Kralchevsky, N. D. Denkov, G. Broze, and A. Mehreteab, J. Colloid Interface Sci. 245, 371 (2002).

[153] P. Poulin, Science 275, 1770 (1997).

[154] S. H. Koenig, Biopolymers 14, 2421 (1975).

[155] E. B. Kim, N. Lockwood, M. Chopra, O. Guzmán, N. L. Abbott, and J. J. de Pablo, Biophys. J. 89, 3141 (2005)

[156] V. K. Aswal and P. S. Goyal, Chem. Phys. Lett. 364, 44 (2002).

[157] E. Antoniou and M. Tsianou, J. Appl. Polym. Sci. 125, 1681 (2012).

[158] S. Ariyaprakai and S. R. Dungan, Langmuir 24, 3061 (2008).

[159] H. Haidara, L. Vonna, and J. Schultz, J. Chem. Phys. 107, 630 (1997).

[160] S. Nakata, H. Komoto, K. Hayashi, and M. Menzinger, J. Phys. Chem. B 104, 3589 (2000).

[161] I. Lagzi, S. Soh, P. J. Wesson, K. P. Browne, and B. A. Grzybowski, J. Am. Chem. Soc. 132, 1198 (2010).

[162] J. Čejková, M. Novák, F. Štěpánek, and M. M. Hanczyc, Langmuir 30, 11937 (2014).

[163] I. D. Couzin, Trends Cogn. Sci. 13, 36 (2009).

[164] J. Taktikos, V. Zaburdaev, and H. Stark, Phys. Rev. E - Stat. Nonlinear, Soft Matter Phys. 84, 1 (2011).

[165] J. Taktikos, V. Zaburdaev, and H. Stark, Phys. Rev. E - Stat. Nonlinear, Soft Matter Phys. 85, 1 (2012).

[166] T. W. Kranz, A. Gelimson, K. Zhao, G. C. L. Wong, and R. Golestanian, Phys. Rev. Lett. 038101, 1 (2016).

[167] A. Sengupta, S. van Teeffelen, and H. Löwen, Phys. Rev. E - Stat. Nonlinear, Soft Matter Phys. 80, 1 (2009).

[168] C. Jin, C. Krüger, and C. C. Maass, in preparation (2016).

[169] A. T. Chwang and T. Y.-T. Wu, J. Fluid Mech. 67, 787 (1975)

[170] K. C. Leptos, J. S. Guasto, J. P. Gollub, A. I. Pesci, and R. E. Goldstein, Phys. Rev. Lett. 103, 1 (2009).

[171] G. Mino, T. E. Mallouk, T. Darnige, M. Hoyos, J. Dauchet, J. Dunstan, R. Soto, Y. Wang, A. Rousselet, and E. Clement, Phys. Rev. Lett. 106, 1 (2011).

[172] J. Dunkel, V. B. Putz, I. M. Zaid, and J. M. Yeomans, Soft Matter 6, 4268 (2010). 
[173] P. Watnick and R. Kolter, J. Bacteriol. 182, 2675 (2000)

[174] M. G. Mazza, J. Phys. D. Appl. Phys. 49, 203001 (2016).

[175] A. P. Berke, L. Turner, H. C. Berg, and E. Lauga, Phys. Rev. Lett. 101, 1 (2008)

[176] V. Kantsler, J. Dunkel, M. Polin, and R. E. Goldstein, Proc. Natl. Acad. Sci. 110, 1187 (2013).

[177] O. Sipos, K. Nagy, R. Di Leonardo, and P. Galajda, Phys. Rev. Lett. 114, 1 (2015).

[178] M. Contino, E. Lushi, I. Tuval, V. Kantsler, and M. Polin, Phys. Rev. Lett. 115, 1 (2015).

[179] P. Galajda, J. Keymer, P. Chaikin, and R. Austin, J. Bacteriol. 189, 8704 (2007).

[180] M. B. Wan, C. J. Olson Reichhardt, Z. Nussinov, and C. Reichhardt, Phys. Rev. Lett. 101, 1 (2008)

[181] J. Tailleur and M. E. Cates, Europhys. Lett. 86, 60002 (2009).

[182] S. E. Hulme, W. R. DiLuzio, S. S. Shevkoplyas, L. Turner, M. Mayer, H. C. Berg, and G. M. Whitesides, Lab Chip 8, 1888 (2008)

[183] M. Mijalkov and G. Volpe, Soft Matter 9, 6376 (2013).

[184] G. Li and J. X. Tang, Phys. Rev. Lett. 103, 1 (2009)

[185] G. J. Li and A. M. Ardekani, Phys. Rev. E - Stat. Nonlinear, Soft Matter Phys. 90, 1 (2014).

[186] W. E. Uspal, M. N. Popescu, S. Dietrich, and M. Tasinkevych, Soft Matter 11, 434 (2015).

[187] S. E. Spagnolie, G. R. Moreno-Flores, D. Bartolo, and E. Lauga, Soft Matter 11, 3396 (2015).

[188] K. Schaar, A. Zöttl, and H. Stark, Phys. Rev. Lett. 115, 1 (2015)

[189] D. Takagi, J. Palacci, A. B. Braunschweig, M. J. Shelley, and J. Zhang, Soft Matter 10, 3 (2014)

[190] C. Krüger, G. Klös, C. Bahr, and C. C. Maass, Phys. Rev. Lett. 117, 048003 (2016)

[191] H. C. Crenshaw, Am. Zool. 36, 608 (1996).

[192] R. Ledesma-Aguilar, H. Löwen, and J. M. Yeomans, Eur. Phys. J. E 35, 70 (2012).

[193] B. ten Hagen, R. Wittkowski, D. Takagi, F. Kümmel, C. Bechinger, and H. Löwen, J. Phys. Condens. Matter 27, 194110 (2015).

[194] W. T. Kranz, A. Gelimson, and R. Golestanian, ArXiv e-prints , 1 (2015), arXiv:1504.06814. 
[195] S. Candau, P. Le Roy, and F. Debeauvais, Mol. Cryst. Liq. Cryst. 23, 283 (1973)

[196] D. Gomez-Diaz, J. M. Navaza, and B. Sanjurjo, J. Chem. Eng. Data 52, 2091 (2007).

[197] J. Happel and H. Brenner, Low Reynolds Number Hydrodynamics (Prentice Hall, NJ, 1965).

[198] C. Krüger, C. Bahr, S. Herminghaus, and C. C. Maass, Eur. Phys. J. E 39, 64 (2016).

[199] A. Zöttl, Hydrodynamics of Microswimmers in Confinement and in Poiseuille Flow, Ph.D. thesis, Technical University Berlin (2014).

[200] I. O. Götze and G. Gompper, Phys. Rev. E - Stat. Nonlinear, Soft Matter Phys. 82, 1 (2010).

[201] N. Aguillon, A. Decoene, B. Fabrèges, B. Maury, and B. Semin, ESAIM Proc. 38, 36 (2013).

[202] M. Doi, Introduction to Polymer Physics (Oxford University Press, 1990).

[203] B. ten Hagen, S. van Teeffelen, and H. Löwen, J. Phys. Condens. Matter 23, 194119 (2011).

[204] J. Bialké, H. Löwen, and T. Speck, Eur. Lett. 103, 30008 (2013).

[205] A. P. Petroff, X.-L. Wu, and A. Libchaber, Phys. Rev. Lett. 158102, 1 (2015).

[206] P. K. Ghosh, V. R. Misko, F. Marchesoni, and F. Nori, Phys. Rev. Lett. 110, 1 (2013).

[207] M. Sandoval and L. Dagdug, Phys. Rev. E - Stat. Nonlinear, Soft Matter Phys. 90, 1 (2014).

[208] M. Kornreich, M. Heymann, S. Fraden, and R. Beck, Lab Chip 14, 3700 (2014).

[209] J. Noh, K. Reguengo De Sousa, and J. P. F. Lagerwall, Soft Matter 12, 367 (2016).

[210] T. Schreiber, Phys. Rev. Lett. 85, 461 (2000).

[211] I. Jenkins, J. C. Crocker, T. Sinno, and C. M. Lewandowski, Soft Matter 11, 1 (2015).

[212] A. P. Leis, S. Schlicher, H. Franke, and M. Strathmann, Appl. Environ. Microbiol. 71, 4801 (2005). 


\section{Acknowledgements}

I could not have finished this $\mathrm{PhD}$ thesis left on my own. Many people contributed to it in one way or another. Sometimes related to science, but often apart from it!

I want to start with acknowledging Prof. Stephan Herminghaus. Thanks for giving me the opportunity of a PhD thesis and for working in the new, enthralling field of active matter in your department. The encounters and scientific discussions always left me inspired and full of new ideas.

Many thanks to Prof. Jörg Enderlein for reviewing my thesis and for staying interested, even though the topic shifted away from his field of expertise. As well I am grateful to the other Professors for joining the defence committee. This includes, Prof. Sarah Köster, Prof. Annette Zippelius and Prof. Christoph Schmidt.

My deepest felt thanks go to Dr. Christian Bahr for introducing me to the world of liquid crystals and surfactant solutions. I appreciate his advice and constant availability to help during my whole PhD. The many discussions helped me a lot in finding my way through all the interesting behaviours our droplets showed, but moreover, picking the ones presented in this thesis and the prove reading cannot be understated.

On the same lines, thanks to Dr. Corinna Maass for an extensive help in the intriguing physics of active systems, paper writing, proof reading and especially Python coding.

To finalize my quartet of supervisors, I want to sincerely thank Dr. Kristian Hantke. For many hours on the confocal microscope unfortunately waisted, since the exploration of the swimming droplets brought me into other domains. Nevertheless, his technical support was invaluable along the way and the many conversations about different topics I would not like to have missed. Thanks as well for being the devil's advocate from now and then.

And where would scientists end without all the help from the administrative and technical staff? My thanks go to Monika Teuteberg and Guido Schriever for always helping with the administrative obstacles one is facing from time to time, while always being cheerful. To Thomas Eggers, not just for keeping the computers running, but much more for all the fun conversations apart from work and the supply with great music. And to Wolf Keiderling for all the help with building small pieces for the experiments.

When spending more than three years at the MPI, there are numerous acquaintances over time. Post-Docs, PhD students, Interns, Undergraduates, everyone leaves an impression. During all the time, I enjoyed working with so many people. I appreciate the help along the way and owe them my gratitude. Many of these people also enriched my stay in Göttingen outside of the Institute. 
In particular: Anupam and Ben, for getting me started with liquid crystals and for introducing me to evenings in Göttingen. Jiseok, Philipp, Birte and Ingmar, for help in the cleanroom, with the microfluidic setup and many related discussions. André, for many discussions about ways to tame the droplets and force them into an analysis scheme. Your help is highly appreciated and your critical proof reading improved the thesis a lot. Chenyu, for many interesting discussions about the auto(chemotactic) behaviour of the droplets. And thanks to many people just for being around and creating the great atmosphere at DCF. Hakam and Gunnar, for sharing an office. Marcin, Arnaud, Paul, Tanya, Julien, Paolo, Christian, Marco, and many more for nice conversations when stumbling into each other in the labs and corridors.

Thanks to René, Britta and Francesco for many great evenings spend or hiking tours in the Harz. It was always a pleasure.

Of course, some people always get mentioned last, although they do not deserve this place in the row. My family always supported me throughout my studies, be it in Bielefeld, Lund or Göttingen. They always backed me up where they could.

Jenny, my loving wife. You supported me whenever necessary and you have been always by my side. Even through thesis writing and proof reading. It is important and relaxing to have someone close, understanding the perils of a PhD student. I'm grateful to have you by my side. 


\section{Eidesstattliche Erklärung}

Hiermit erkläre ich, dass ich die vorliegende Arbeit selbstständig angefertigt, nicht anderweitig zu Prüfungszwecken vorgelegt und keine anderen als die angegebenen Hilfsmittel verwendet habe. Sämtliche wissentlich verwendeten Textausschnitte, Zitate oder Inhalte anderer Verfasser wurden ausdrücklich als solche gekennzeichnet. 



\section{Curriculum Vitae}

\section{Personal Details}

$\begin{array}{ll}\text { Name: } & \text { Carsten Krüger } \\ \text { Date of birth: } & 21.05 .1987 \\ \text { Place of birth: } & \text { Bielefeld, Germany } \\ \text { Civil Status: } & \text { Married } \\ \text { Adress: } & \text { Goerdelerweg 6, 37075 Göttingen } \\ \text { Nationality: } & \text { German }\end{array}$

\section{Education}

$06 / 2013-11 / 2016$

Doctoral research at the Max Planck Institute for

Dynamics and Self-Organization, Göttingen, Germany

Supervisors: Prof. Dr. Stephan Herminghaus,

Dr. Christian Bahr, Dr. Corinna C. Maass

10/2010 - 05/2013 Master of Science in Physics/Nanoscience

Bielefeld University, Germany

Supervisor: Prof. Dr. Thomas Huser

10/2007 - 10/2010 Bachelor of Science in Physics

Bielefeld University, Germany

Supervisor: Dr. Mark Schüttpelz

\section{List of publications related to doctoral dissertation}

1. Herminghaus, S.; Maass, C. C.; Krüger, C.; Thutupalli, S.; Goehring, L.; Bahr, Ch.: "Interfacial mechanisms in active emulsions", Soft Matter 10, 7008-7022 (2014)

2. Maass, C. C.; Krüger, C.; Herminghaus, S.; Bahr, Ch.: "Swimming droplets", Annual Review of Condensed Matter Physics 7 171-193 (2016)

3. Krüger, C.; Bahr, Ch.; Herminghaus, S.; Maass, C. C.: "Dimensionality matters in the collective behaviour of active emulsions", The European Physical Journal E 39(6) 64 (2016)

4. Krüger, C.; Klös, G.; Bahr, Ch.; Maass, C. C.: "Curling liquid crystal microswimmers: A cascade of spontaneous symmetry breaking", Physical Review Letters 107048003 (2016) 